\title{
A home-based program to manage concerns about falls
}

Citation for published version (APA):

Dorresteijn, T. (2016). A home-based program to manage concerns about falls: feasibility, effects and costs of a cognitive behavioral approach in community-dwelling, frail older people. [Doctoral Thesis, Maastricht University]. Datawyse / Universitaire Pers Maastricht. https://doi.org/10.26481/dis.20160512td

Document status and date:

Published: 01/01/2016

DOI:

10.26481/dis.20160512td

Document Version:

Publisher's PDF, also known as Version of record

\section{Please check the document version of this publication:}

- A submitted manuscript is the version of the article upon submission and before peer-review. There can be important differences between the submitted version and the official published version of record.

People interested in the research are advised to contact the author for the final version of the publication, or visit the DOI to the publisher's website.

- The final author version and the galley proof are versions of the publication after peer review.

- The final published version features the final layout of the paper including the volume, issue and page numbers.

Link to publication

\footnotetext{
General rights rights.

- You may freely distribute the URL identifying the publication in the public portal. please follow below link for the End User Agreement:

www.umlib.nl/taverne-license

Take down policy

If you believe that this document breaches copyright please contact us at:

repository@maastrichtuniversity.nl

providing details and we will investigate your claim.
}

Copyright and moral rights for the publications made accessible in the public portal are retained by the authors and/or other copyright owners and it is a condition of accessing publications that users recognise and abide by the legal requirements associated with these

- Users may download and print one copy of any publication from the public portal for the purpose of private study or research.

- You may not further distribute the material or use it for any profit-making activity or commercial gain

If the publication is distributed under the terms of Article $25 \mathrm{fa}$ of the Dutch Copyright Act, indicated by the "Taverne" license above, 


\section{A home-based program to manage concerns about falls}

Feasibility, effects and costs of a cognitive behavioral approach in community-dwelling, frail older people

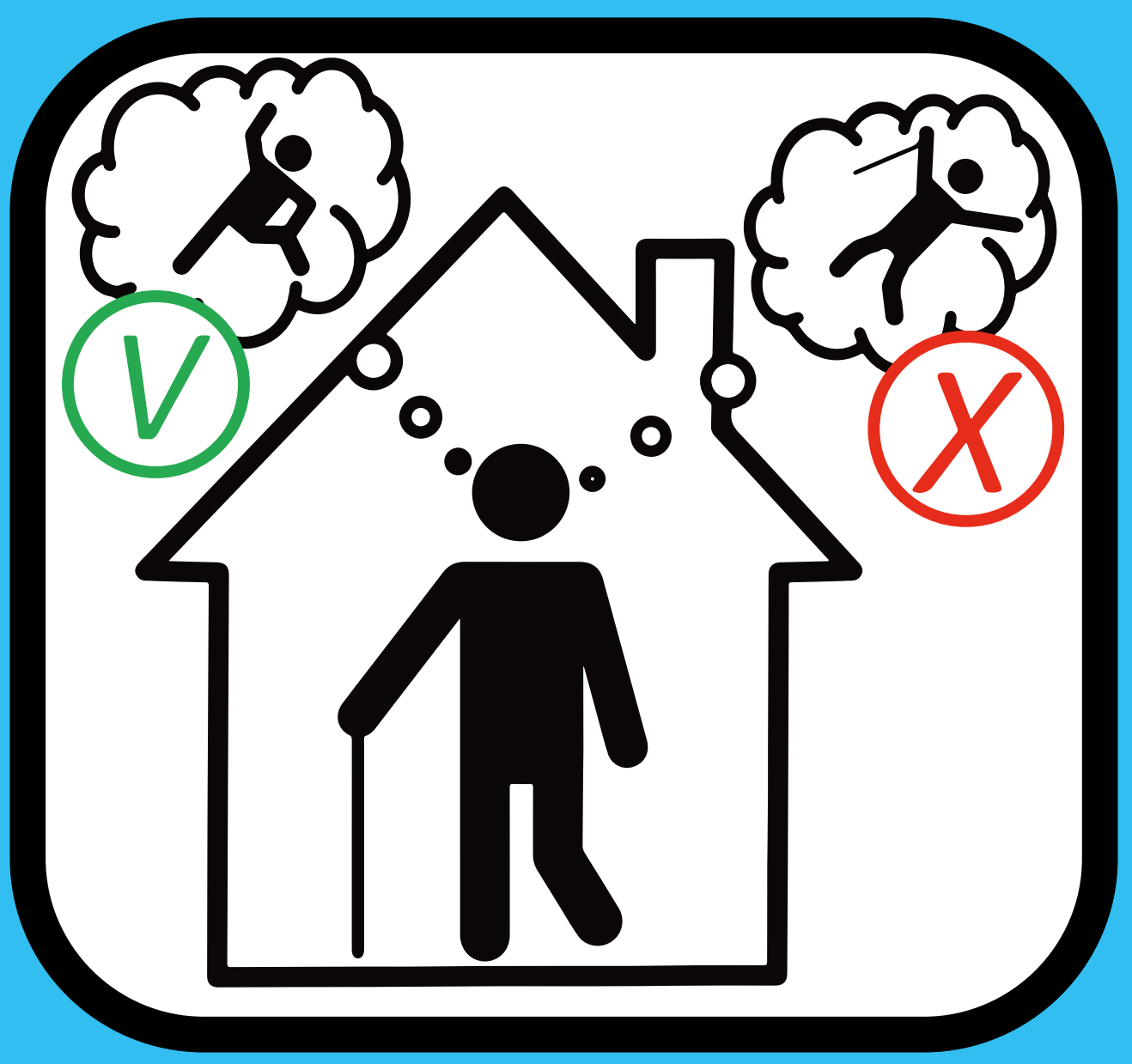

Tanja Dorresteijn 
Cover and illustrations: Michiel Ritzen

Printing: Datawyse | Universitaire Pers Maastricht

(C) Tanja Dorresteijn, Maastricht 2016

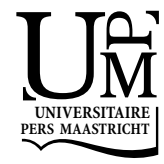

ISBN 9789461595553

The studies presented in this thesis were performed at the Department of Health Services Research, School for Public Health and Primary Care (CAPHRI), Maastricht University. CAPHRI is part of the Netherlands School of Primary Care Research (CaRe), which has been acknowledged since 1995 by the Royal Netherlands Academy of Arts and Sciences (KNAW).

Funded by the Netherlands Organization for Health Research and Development (ZonMw nr. 120610001). 


\title{
A home-based program to manage concerns about falls
}

\section{Feasibility, effects and costs of a cognitive behavioral approach in community-dwelling, frail older people}

\author{
PROEFSCHRIFT \\ ter verkrijging van de graad van doctor aan de Universiteit Maastricht \\ op gezag van de Rector Magnificus, Prof. Dr. L.L.G. Soete \\ volgens het besluit van het College van Decanen, \\ in het openbaar te verdedigen \\ op donderdag 12 mei 2016 om 12.00 uur \\ door \\ Tanja Anna Cornelia Dorresteijn \\ Geboren op 29 oktober 1981 te Utrecht
}


Promotores:

Prof. dr. G.I.J.M. Kempen

Prof. dr. J.W.S. Vlaeyen

Copromotor:

Dr. G.A.R. Zijlstra

Beoordelingscommissie:

Prof. dr. G. Kok (voorzitter)

Prof. dr. F.J.V.M. Buntinx (KU Leuven, België)

Prof. dr. C.D. Dirksen

Prof. dr. S. Iwarsson (Lund University, Sweden)

Prof. dr. T. van der Weijden 


\section{Contents}

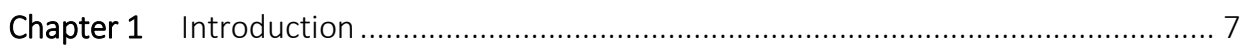

Chapter 2 Older people's preferences regarding program formats for managing concerns about falls

Published in Age and ageing. 2012;41(4):474-481

Chapter 3 Evaluating an in-home multicomponent cognitive behavioral program to manage concerns about falls and associated activity avoidance in frail community-dwelling older people: design of a randomized controlled trial Published in BMC Health services research. 2010;11:228.

Chapter 4 Feasibility of a nurse-led in-home cognitive behavioral program to manage concerns about falls in frail older people: a process evaluation

Published in Research in nursing \& health. 2013;36(3):257-270 ...... 59

Chapter 5 Effectiveness of a home-based cognitive behavioral program to manage concerns about falls in community-dwelling, frail older people: results of a randomized controlled trial Published in BMC Geriatrics. 2016;16(1):2.

Chapter 6 Cost-effectiveness of a home-based program to reduce concerns about falls in frail, community-dwelling older people: evidence from a randomized controlled trial

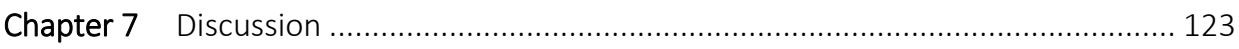

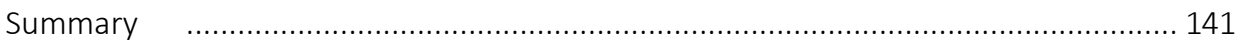

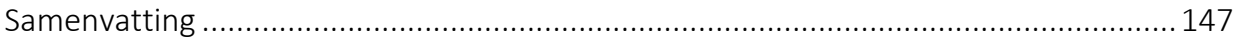

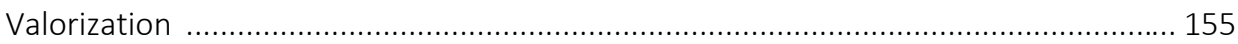

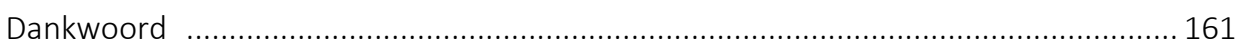

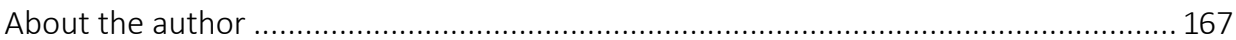

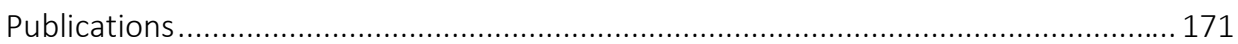



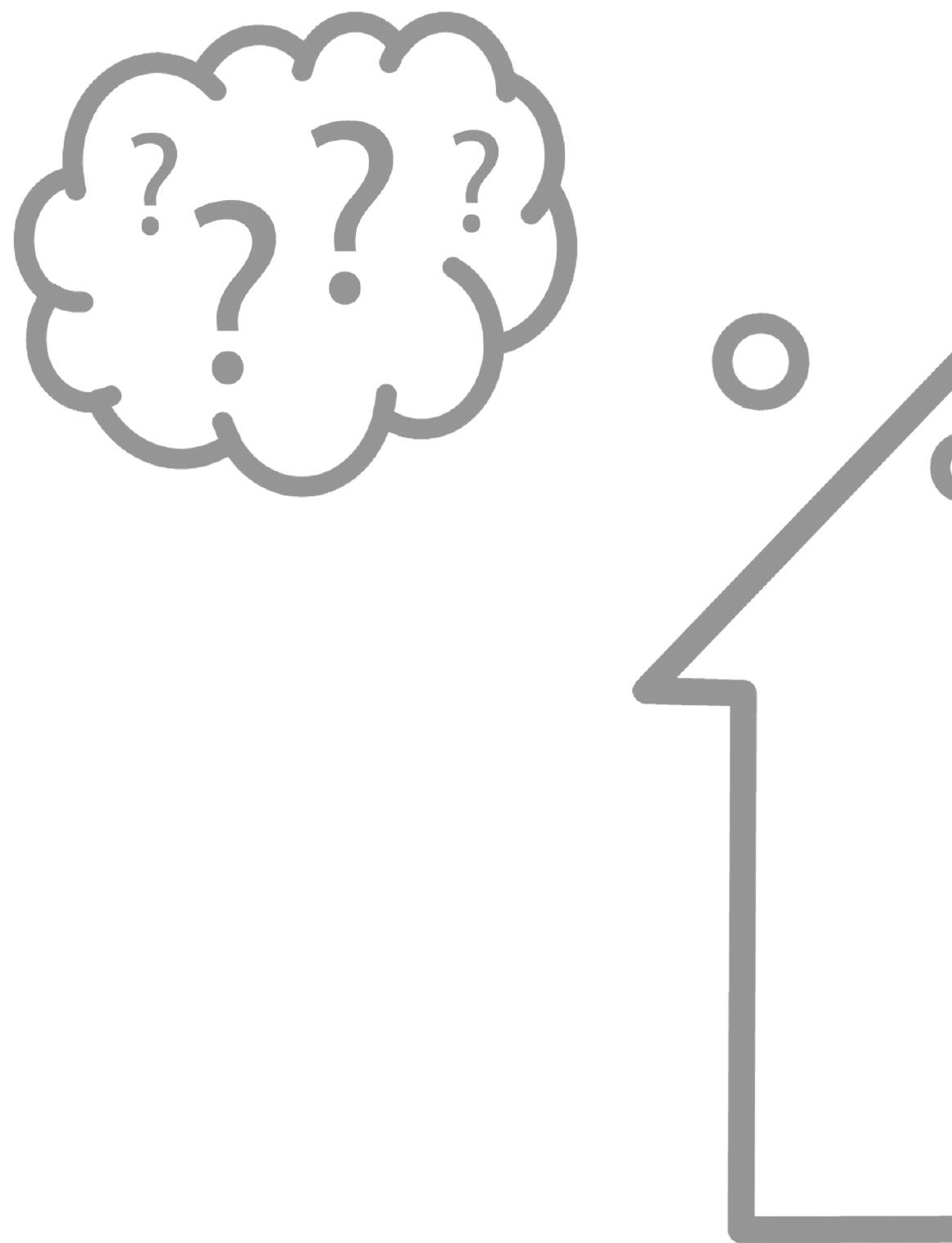


\section{Chapter 1}

Introduction 



\section{Preface}

One of the most common fear among older people is the fear of falling. ${ }^{1}$ This particular fear associated with concerns about potential falls, can have great impact on daily life and be a threat to independent living of those who it affects. ${ }^{2}$ At the same time, national policies worldwide encourage and facilitate older people to live independently as long as possible. ${ }^{3-5}$ This policy reflects the preference of many older people themselves ${ }^{6}$ and is in line with the need to keep the healthcare costs affordable with the demographic trend of an increasing proportion of people aged 65 years and over. ${ }^{3}$ Therefore, interventions who support older people in their independence are warranted. Selfmanagement skills, like problem solving, resource utilization and action planning, could be a powerful tool in order to achieve this goal by community-dwelling older people. ${ }^{7}$

Evidence based programs for frail older people with a focus on concerns about falls and supporting self-management skills are scarce. Hence, the aim of this thesis is the development and evaluation of a home-based, cognitive behavioral program to manage concerns about falls and by extension to contribute to the independence of communitydwelling, frail older people. The main objectives of the study are to examine the feasibility and the (cost-)effectiveness of this novel self-management program with concerns about falls as primary outcome.

\section{Conceptualization of concerns about falls}

\section{Definitions}

Psychological and behavioral consequences of a fall were first introduced as a specific phobia, ptophobia ${ }^{8}$, or as the post-fall syndrome ${ }^{9}$. These two medically-oriented concepts were based on the observation that patients can develop intense fear of standing or walking as a result of a fall, even in the absence of any neuromuscular impairment that could be responsible for these symptoms. ${ }^{8}$ Later, Tinetti and colleagues showed that fear of falling is not restricted to those who had recently experienced a fall, but also extends to individuals who never fell. ${ }^{10}$ They presented the following definition of fear of falling: "a lasting concern about falling that leads to an individual avoiding activities that he/she remains capable of performing". ${ }^{11}$

In order to measure fear of falling, Tinetti and colleagues introduced the concept of fall-related self-efficacy ${ }^{12}$, based on the social cognitive theory of Bandura ${ }^{13}$. In this context fall-related self-efficacy implies the confidence in one's ability to perform activities of daily living (ADL) without falling. ${ }^{12}$ The general concept of self-efficacy is grounded on theoretical assumptions about the cognitive process that underlies emotions, i.e. performance accomplishments, vicarious experience, verbal persuasion, and physiological states. ${ }^{14}$ Therefore, numerous assessment strategies have been developed to assess self-efficacy in a variety of health-related domains. In addition, self-efficacy has 
shown to be associated with functional limitations as persons with low perceived selfefficacy about an activity tend to avoid that activity. ${ }^{15}$

Over time more concepts to define or measure fall-related psychological constructs were presented, such as balance confidence ${ }^{16}$, self-restricted avoidance of activities ${ }^{17}$, feared consequences of falling ${ }^{18}$, and concerns about falls ${ }^{19}$ (see Table 1). Although these constructs are correlated, they are not the same and therefore do not necessarily measure the same construct. ${ }^{20,21}$ Moreover, recent research clearly demonstrates that fall-related self-efficacy should not be used interchangeably with fear of falling. ${ }^{22,23}$ For instance, fall-related self-efficacy and balance confidence are mostly overlapping constructs. ${ }^{24}$ On the other hand, fear of falling is more similar to ptophobia or post-fall syndrome and concerns about falls. The latter is related to fear, but is less intense and less emotional, and therefore may be more socially acceptable for older people to disclose. ${ }^{19}$ In this thesis the use of the term concerns about falls is preferred instead of fear of falling.

Table 1 Fall-related psychological constructs in historical order.

\begin{tabular}{|c|c|}
\hline Fall-related psychological constructs & Description \\
\hline Ptophobia $^{8}$ or post-fall syndrome ${ }^{9}$ & $\begin{array}{l}\text { Intense fear of standing or walking as a result of a fall without evidence } \\
\text { of a neuromuscular impairment causing inability to walk }\end{array}$ \\
\hline Fall related self-efficacy & $\begin{array}{l}\text { Confidence in one's ability to perform activities of daily living (ADL) } \\
\text { without falling }{ }^{12}\end{array}$ \\
\hline Fear of falling & $\begin{array}{l}\text { "A lasting concern about falling that leads to an individual avoiding } \\
\text { activities that he/she remains capable of performing, and therefore not } \\
\text { restricted to those who had recently experienced a fall“" }\end{array}$ \\
\hline Balance confidence & $\begin{array}{l}\text { Confidence in one's ability to maintain balance and remain steady } \\
\text { whilst performing ADLs }\end{array}$ \\
\hline $\begin{array}{l}\text { Self-restricted avoidance of } \\
\text { activities }\end{array}$ & $\begin{array}{l}\text { Avoiding activities, which one is still capable of performing, due to fear } \\
\text { of falling }{ }^{17}\end{array}$ \\
\hline $\begin{array}{l}\text { Feared consequences of falling or } \\
\text { outcome expectancy }\end{array}$ & $\begin{array}{l}\text { Feared loss of functional independence and damage to identity due to } \\
\text { a fall }\end{array}$ \\
\hline Concerns about falls & A term that is closely related to fear, but is less intense and emotional ${ }^{19}$ \\
\hline
\end{tabular}

\section{Theoretical models}

Normally is fear an adaptive response of the individual during exposure to dangerous situations. ${ }^{25}$ The "Three-System-Model" of fear and emotion of Rachman ${ }^{26}$ is a widely used model to explain this response. According to this model fear consists of three components: (a) the physiological (e.g., increased autonomic reactivity, like rapid heartbeat), (b) the behavioral (e.g., walking in a very slow speed to prevent a fall or escaping 
from and avoiding an activity), and (c) the cognitive component (e.g., one's subjective estimation of the level of danger and ability to avoid a fall). ${ }^{26}$ In this light, concerns about falls could be an accurate estimation of the level of fall risk. However, a study of Delbaere and colleagues demonstrated that there was a mismatch between perceived and physiological fall risk among older people in almost one-third of their study population. ${ }^{27}$ In their study perceived fall risk was estimated with the Falls Efficacy Scale International $^{19}$ and physiological fall risk was estimated with a physiological profile assessment (including vision, muscle strength, proprioception, and balance). High levels of concerns about falls in the group who had a low physiological fall risk were related to depressive symptoms and decreased executive functioning. On the other hand, a positive outlook on life and maintenance of physical activity and community participation were protective for falling in the group who had a high physiological fall risk, but low levels of concerns about falls.

Only a few theoretical models are identified to explain the origin and consequences of concerns about falls. For a long period, the traditional fear-avoidance model was used to describe how concerns about falls lead to falls. This model was first introduced by Lethem and colleagues to explain how people developed chronic musculoskeletal pain despite the absence of pathology as a result of avoidance behavior based on fear. ${ }^{28}$ By avoiding the situation or activity, the fear can be controlled and a lack of pain reinforces this behavior, however, on the long run, avoidance behavior can lead to disability and depression. In an adapted version, also called downward spiral, concerns about falls lead to a lack of balance confidence (efficacy) and subsequently to avoidance of activities that in turn leads to physical deconditioning and a poorer balance performance, and ultimately, to falls. ${ }^{29}$

An alternative model was more recently proposed by Hadjistavropoulos and colleagues. ${ }^{30}$ In this model the relationship between concerns about falls and falls is more complex and multifactorial. Other risk factors, for example, visual problems, demographic characteristics and realistic appraisal of one's own abilities can influence balance performance as well as falls. Past falls and beliefs have a direct influence on the level of concerns about fall, while the role of activity avoidance as mediator between concerns about falls and falls remains unclear. Although it is evident that concerns about falls and avoidance of activities go often hand in hand ${ }^{17,31-34}$ and that avoidance can lead to functional decline $e^{23,31,35}$, there is no clear evidence for a causal relationship between avoidance behavior and falls ${ }^{23,35}$. This theoretical framework is supported by a recent longitudinal study testing the relation between injurious falls and concerns about falls. ${ }^{36}$ In this study, various predictors were found for both outcomes. Age was a predictor for both injurious falls and concerns about falls. Gait and balance predicted injurious falls, whereas decreasing social engagement was one of the strongest predictors for concerns about falls. ${ }^{36}$ These findings suggest that falls and concerns about falls are different phenomena, and need their own assessment and therapeutic approach. 


\section{Prevalence, risk factors and consequences}

Concerns about falls are widespread among older persons. Prevalence rates between $20 \%$ and $85 \%$ are reported, depending on the target group that is included and the measures being used. ${ }^{2}$ In the Netherlands, a prevalence rate of $54 \%$ was observed in the general population of community-dwelling older people of 70 years of age or older. ${ }^{37}$ Risk factors that are strongly associated with concerns about falls are female gender, impaired physical function, and the use of a walking aid. Less strongly associated are the history of falls, self-rated health, multiple medications, psychotropic drugs, depression, and anxiety. ${ }^{38}$ Consequences of concerns about falls can be falls, avoidance of activity, a decreased balance performance, decreased mobility, lower social participation, functional decline, low quality of life, and loss of independence. . $3,27,29,39,40^{-10}$

\section{Programs to manage concerns about falls}

Given the high prevalence rate and potentially adverse consequences of concerns about falls and associated avoidance behavior, effective interventions are needed. Interventions directly or indirectly targeting concerns about falls can be roughly classified into exercise based or multifactorial interventions. ${ }^{41-43}$ Exercise based interventions are for instance strength or balance training or tai chi; multifactorial interventions usually are a combination of exercise, education, fall risk assessment or cognitive behavioral therapy. ${ }^{41,42}$ The reviews of Zijlstra and colleagues ${ }^{42}$, Bula and colleagues ${ }^{41}$, and particularly the more recent review and meta-analyses of Kendrick and colleagues ${ }^{43}$ demonstrate that there is little evidence that physical exercise alone can reduce concerns about falls. However, there is increasing evidence that a combination of physical exercise with a cognitive behavioral approach can be (cost)effective in decreasing concerns about falls and related avoidance behavior. ${ }^{44-52}$

A cognitive behavioral group program named 'A Matter of Balance' is theorybased $^{53,54}$ and inspired by the work of Bandura ${ }^{13}$, Beck ${ }^{55}$ and Meichenbaum ${ }^{56}$. Principles of the program are: 1) identifying and restructuring misconceptions about falls and fall risk; 2) setting realistic personal goals for increasing activity levels and safe behavior; and 3) promoting the uptake of old and new daily life activities that were avoided due to concerns about falls. The original U.S. version was adapted to the Dutch healthcare setting ${ }^{57}$ and showed even more robust effects on concerns about falls and associated activity avoidance in the Netherlands ${ }^{45}$. Moreover, the Dutch version of 'A Matter of Balance' showed to be effective in increasing control beliefs, self-efficacy beliefs, outcomes expectations and social interactions. ${ }^{58}$ Nevertheless, $42 \%$ of the participants attended less than five of the eight intervention sessions. The most common reason reported to explain the dropout were health problems. ${ }^{59}$ It seemed that, in particular, frail older people refrained from attending the group program. Other group programs 
that explicitly address concerns about falls, and target frail older people living in the community observed similar outcomes. ${ }^{44,60}$ Yet, this frail group who has in general higher levels of disability, lower quality of life and poorer executive functioning, have an additional risk to develop an overestimated (unrealistic) perceived risk of falling. ${ }^{27}$ To meet the needs and capabilities of this target group, a home-based format of 'A Matter of Balance' could be a good alternate for the group format.

\section{Objectives}

This thesis has three main objectives. First, it aims to explore which format(s) of a program that primarily focuses on managing concerns about falls are preferred by community-dwelling older people. Second, to study in a process evaluation the feasibility and acceptability of a home-based, cognitive behavioral program to manage concerns about falls in frail, community-dwelling, older people. Third, to analyze whether the newly developed program is (cost-)effective with respect to the reduction of concerns about falls when compared to care as usual.

\section{Outline}

The aims of this thesis are subdivided in different chapters with the following outline.

Little is known regarding the willingness of older people to participate in a program aimed at managing concerns about falls and whether this willingness to participate is related to the format in which the program is offered - for instance, via telephone, television or the internet. In Chapter 2 the results of a cross-sectional study that explores the preferences of community-dwelling older people regarding six different formats of a program that primarily focuses on managing concerns about falls are described. Data was obtained during the screening procedure for the selection of participants for our trial to evaluate a home-based, cognitive behavioral program.

Concerns about falls are often based on misconceptions of what may cause a fall, and they can be associated with avoidance behavior. Cognitive behavioral principles can therefore be a suitable strategy to reduce this problem by modifying patterns of thoughts (cognition) and actions (behavior) that contribute to the concern and avoidance. Although an effective group program is available in the Netherlands, it appears that especially frail older people are not participating in the group program. To reach this target group, as well as people who prefer a home-based approach, the group program was adapted to a home-based program while preserving its essential components. The process of the development and the final content of the program, as well as a delineation of the design of the evaluation study can be found in Chapter 3. 
A process evaluation conducted alongside a randomized controlled trial is seen as crucial for a correct interpretation of the outcomes, and the results can be used as input for intervention improvement. Therefore, we assessed our newly developed program with respect to participation of the target population in the program (reach); implementation of the program as planned (fidelity); participants' engagement in and receptivity to the program (dose received-exposure); participants' and facilitators' satisfaction with the program (dose received-satisfaction); and aspects of the program that need improvement prior to nationwide implementation (barriers). The results of our feasibility study are described in Chapter 4.

In a randomized controlled trial, we tested whether our home-based, cognitive behavioral program was effective in reducing concerns about falls when compared to care as usual. Secondary outcomes included the effects of the program on activity avoidance, restrictions in daily life, and falls. In Chapter 5 the results on these outcomes are reported.

The cost-effectiveness of a health care innovation is becoming dominant in the discussion of health care cost control. Information of the ratio of costs and effects can be helpful to make balanced decisions about which interventions should be made available and implemented in health care. Results from an economic evaluation of our home-based, cognitive behavioral program are reported in Chapter 6.

A general discussion is presented in Chapter 7. This discussion includes the main findings of the studies presented in this thesis, theoretical and methodological considerations, and the implications for clinical practice and future research. 


\section{References}

1. Howland J, Peterson EW, Levin WC, Fried L, Pordon D, Bak S: Fear of falling among the communitydwelling elderly. J Aging Health. 1993, 5(2):229-243.

2. Scheffer AC, Schuurmans MJ, van Dijk N, van der Hooft T, de Rooij SE: Fear of falling: measurement strategy, prevalence, risk factors and consequences among older persons. Age Ageing. 2008, 37(1):1924.

3. Gezondheidsraad: Preventie bij ouderen: focus op zelfredzaamheid. Den Haag: Gezondheidsraad; 2009.

4. UK Government: Government response to the house of lords select committee on public service and demographic change report of session 2012-13: 'ready for ageing?'. London: www.gov.uk; 2013.

5. Community Living Council: U.S. Department of health and human services strategic plan for community living, FY 2014-FY 2018. Washington, DC: Department of health and human services; 2014.

6. Robinson SM, Ni Bhuachalla B, Ni Mhaille B, Cotter PE, O'Connor M, O'Keeffe ST: Home, please: a conjoint analysis of patient preferences after a bad hip fracture. Geriatr Gerontol Int. 2015, 15(10):11651170 .

7. Lorig KR, Holman H: Self-management education: history, definition, outcomes, and mechanisms. Ann Behav Med. 2003, 26(1):1-7.

8. Bhala RP, O'Donnell J, Thoppil E: Ptophobia. Phobic fear of falling and its clinical management. Phys Ther. 1982, 62(2):187-190.

9. Murphy J, Isaacs B: The post-fall syndrome. A study of 36 elderly patients. Gerontology. 1982, 28(4):265270.

10. Tinetti ME, Speechley M, Ginter SF: Risk-Factors for falls among elderly persons living in the community. N Engl J Med. 1988, 319(26):1701-1707.

11. Tinetti ME, Powell L: Fear of falling and low self-efficacy - a cause of dependence in elderly persons. $J$ Gerontol. 1993, 48:35-38.

12. Tinetti ME, Richman D, Powell L: Falls efficacy as a measure of fear of falling. J Gerontol. 1990, 45(6):P239-P243.

13. Bandura A: self-efficacy: toward a unifying theory of behavioral change. Psychol Rev. 1977, 84(2):191215.

14. Bandura A: social foundations of thought and action: a social cognitive theory. Englewood Cliffs, N.J.: Prentice-Hall; 1986.

15. Bandura A: Self-efficacy mechanism in human agency. Am Psychol. 1982, Vol 37(2):122-147.

16. Powell LE, Myers AM: The activities-specific balance confidence (ABC) scale. J Gerontol A Biol Sci Med Sci. 1995, 50A(1):M28-34.

17. Lachman ME, Howland J, Tennstedt S, Jette A, Assmann S, Peterson EW: Fear of falling and activity restriction: the survey of activities and fear of falling in the elderly (SAFE). J Gerontol B Psychol Sci Soc Sci. 1998, 53(1):P43-50.

18. Yardley L, Smith $\mathrm{H}$ : A prospective study of the relationship between feared consequences of falling and avoidance of activity in community-living older people. Gerontologist. 2002, 42(1):17-23.

19. Yardley L, Beyer N, Hauer K, Kempen G, Piot-Ziegler C, Todd C: Development and initial validation of the falls efficacy scale-international (FES-I). Age Ageing. 2005, 34(6):614-619.

20. Moore DS, Ellis R: Measurement of fall-related psychological constructs among independent-living older adults: a review of the research literature. Aging Ment Health. 2008, 12(6):684-699.

21. Jorstad EC, Hauer K, Becker C, Lamb SE, ProFa NEG: Measuring the psychological outcomes of falling: a systematic review. J Am Geriatr Soc. 2005, 53(3):501-510.

22. Li FZ, McAuley E, Fisher KJ, Harmer P, Chaumeton N, Wilson NL: Self-efficacy as a mediator between fear of falling and functional ability in the elderly. J Aging Health. 2002, 14(4):452-466.

23. Hadjistavropoulos T, Martin RR, Sharpe D, Lints AC, McCreary DR, Asmundson GJ: A longitudinal investigation of fear of falling, fear of pain, and activity avoidance in community-dwelling older adults. $J$ Aging Health. 2007, 19(6):965-984. 
24. Hotchkiss A, Fisher A, Robertson R, Ruttencutter A, Schuffert J, Barker DB: Convergent and predictive validity of three scales related to falls in the elderly. Am J Occup Ther. 2004, 58(1):100-103.

25. Sapolsky RM: Why zebras don't get ulcers, 3rd edn. New York: Times Books; 2004.

26. Rachman S: Fear and courage. San Francisco: Freeman; 1978.

27. Delbaere K, Close JC, Brodaty H, Sachdev P, Lord SR: Determinants of disparities between perceived and physiological risk of falling among elderly people: cohort study. BMJ. 2010, 341:c4165.

28. Lethem J, Slade PD, Troup JD, Bentley G: Outline of a fear-avoidance model of exaggerated pain perception--I. Behav Res Ther. 1983, 21(4):401-408.

29. Friedman SM, Munoz B, West SK, Rubin GS, Fried LP: Falls and fear of falling: which comes first? A longitudinal prediction model suggests strategies for primary and secondary prevention. J Am Geriatr Soc. 2002, 50(8):1329-1335.

30. Hadjistavropoulos T, Delbaere K, Fitzgerald TD: Reconceptualizing the role of fear of falling and balance confidence in fall risk. J Aging Health. 2011, 23(1):3-23.

31. Deshpande N, Metter EJ, Lauretani F, Bandinelli S, Guralnik J, Ferrucci L: Activity restriction induced by fear of falling and objective and subjective measures of physical function: a prospective cohort study. $J$ Am Geriatr Soc. 2008, 56(4):615-620.

32. Li F, Fisher KJ, Harmer P, McAuley E, Wilson NL: Fear of falling in elderly persons: association with falls, functional ability, and quality of life. J Gerontol B Psychol Sci Soc Sci. 2003, 58(5):P283-290.

33. Kressig RW, Wolf SL, Sattin RW, O'Grady M, Greenspan A, Curns A, Kutner M: Associations of demographic, functional, and behavioral characteristics with activity-related fear of falling among older adults transitioning to frailty. J Am Geriatr Soc. 2001, 49(11):1456-1462.

34. Fletcher PC, Guthrie DM, Berg K, Hirdes JP: Risk factors for restriction in activity associated with fear of falling among seniors within the community. J Patient Saf. 2010, 6(3):187-191.

35. Delbaere K, Crombez G, Vanderstraeten G, Willems T, Cambier D: Fear-related avoidance of activities, falls and physical frailty. A prospective community-based cohort study. Age Ageing. 2004, 33(4):368-373.

36. Clemson L, Kendig H, Mackenzie L, Browning C: Predictors of injurious falls and fear of falling differ: an 11-year longitudinal study of incident events in older people. J Aging Health. 2015, 27(2):239-256.

37. Zijlstra GA, van Haastregt JC, van Eijk JT, van Rossum E, Stalenhoef PA, Kempen GI: Prevalence and correlates of fear of falling, and associated avoidance of activity in the general population of communityliving older people. Age Ageing. 2007, 36(3):304-309.

38. Denkinger MD, Lukas A, Nikolaus T, Hauer K: Factors associated with fear of falling and associated activity restriction in community-dwelling older adults: a systematic review. Am J Geriatr Psychiatry. 2015, 23(1):72-86.

39. Cumming RG, Salkeld G, Thomas M, Szonyi G: Prospective study of the impact of fear of falling on activities of daily living, SF-36 scores, and nursing home admission. J Gerontol A Biol Sci Med Sci. 2000, 55(5):M299-305.

40. van der Meulen E, Zijlstra GA, Ambergen T, Kempen GI: Effect of fall-related concerns on physical, mental, and social function in community-dwelling older adults: a prospective cohort study. J Am Geriatr Soc. 2014, 62(12):2333-2338.

41. Bula CJ, Monod S, Hoskovec C, Rochat S: Interventions aiming at balance confidence improvement in older adults: an updated review. Gerontology. 2011, 57(3):276-286.

42. Zijlstra GA, van Haastregt JC, van Rossum E, van Eijk JT, Yardley L, Kempen GI: Interventions to reduce fear of falling in community-living older people: a systematic review. J Am Geriatr Soc. 2007, 55(4):603615.

43. Kendrick D, Kumar A, Carpenter H, Zijlstra GAR, Skelton DA, Cook JR, Stevens Z, Belcher CM, Haworth D, Gawler SJ et al: Exercise for reducing fear of falling in older people living in the community. Cochrane $D b$ Syst Rev. 2014, 11.

44. Tennstedt S, Howland J, Lachman M, Peterson E, Kasten L, Jette A: A randomized, controlled trial of a group intervention to reduce fear of falling and associated activity restriction in older adults. J Gerontol B Psychol Sci Soc Sci. 1998, 53(6):P384-392. 
45. Zijlstra GA, van Haastregt JC, Ambergen T, van Rossum E, van Eijk JT, Tennstedt SL, Kempen GI: Effects of a multicomponent cognitive behavioral group intervention on fear of falling and activity avoidance in community-dwelling older adults: results of a randomized controlled trial. J Am Geriatr Soc. 2009, 57(11):2020-2028.

46. Zijlstra GA, van Haastregt JC, Du Moulin MF, de Jonge MC, van der Poel A, Kempen GI: Effects of the implementation of an evidence-based program to manage concerns about falls in older adults. Gerontologist. 2013, 53(5):839-849.

47. Healy TC, Peng C, Haynes MS, McMahon EM, Botler JL, Gross L: The feasibility and effectiveness of translating a matter of balance into a volunteer lay leader model. J App/ Gerontol. 2008, 27(1):34-51.

48. Ory MG, Smith ML, Wade A, Mounce C, Wilson A, Parrish R: Implementing and disseminating an evidence-based program to prevent falls in older adults, Texas, 2007-2009. Prev Chronic Dis. 2010, 7(6):A130.

49. Smith ML, Jiang L, Ory MG: Falls efficacy among older adults enrolled in an evidence-based program to reduce fall-related risk: sustainability of individual benefits over time. Fam Community Health. 2012, 35(3):256-263.

50. Ullmann G, Williams HG, Plass CF: Dissemination of an evidence-based program to reduce fear of falling, South Carolina, 2006-2009. Prev Chronic Dis. 2012, 9:E103.

51. Page TF, Batra A, Palmer R: Cost analysis of a community-based fall prevention program being delivered in South Florida. Fam Community Health. 2012, 35(3):264-270.

52. van Haastregt JC, Zijlstra GA, Hendriks MR, Goossens ME, van Eijk JT, Kempen GI: Cost-effectiveness of an intervention to reduce fear of falling. Int J Technol Assess Health Care. 2013, 29(3):219-226.

53. Lachman ME, Jette A, Tennstedt S, Howland J, Harris BA, Peterson E: A cognitive-behavioural model for promoting regular physical activity in older adults. Psychol Health Med. 1997, 2(3):251-261.

54. Peterson EW: Using cognitive behavioral strategies to reduce fear of falling: A matter of balance. Generations. 2002, 26(4):53-59.

55. Beck A: Cognitive therapy of depression. New York: Guilford Press; 1979.

56. Meichenbaum D: Cognitive behavioural modification: an integrative approach. New York: Plenum; 1977.

57. Zijlstra GA, Tennstedt SL, van Haastregt JC, van Eijk JT, Kempen GI: Reducing fear of falling and avoidance of activity in elderly persons: the development of a Dutch version of an American intervention. Patient Educ Couns. 2006, 62(2):220-227.

58. Zijlstra GA, van Haastregt JC, van Eijk JT, de Witte LP, Ambergen T, Kempen GI: Mediating effects of psychosocial factors on concerns about falling and daily activity in a multicomponent cognitive behavioral group intervention. Aging Ment Health. 2011, 15(1):68-77.

59. van Haastregt JC, Zijlstra GA, van Rossum E, van Eijk JT, de Witte LP, Kempen GI: Feasibility of a cognitive behavioural group intervention to reduce fear of falling and associated avoidance of activity in community-living older people: a process evaluation. BMC Health Serv Res. 2007, 7:156.

60. Sattin RW, Easley KA, Wolf SL, Chen Y, Kutner MH: Reduction in fear of falling through intense tai chi exercise training in older, transitionally frail adults. J Am Geriatr Soc. 2005, 53(7):1168-1178. 

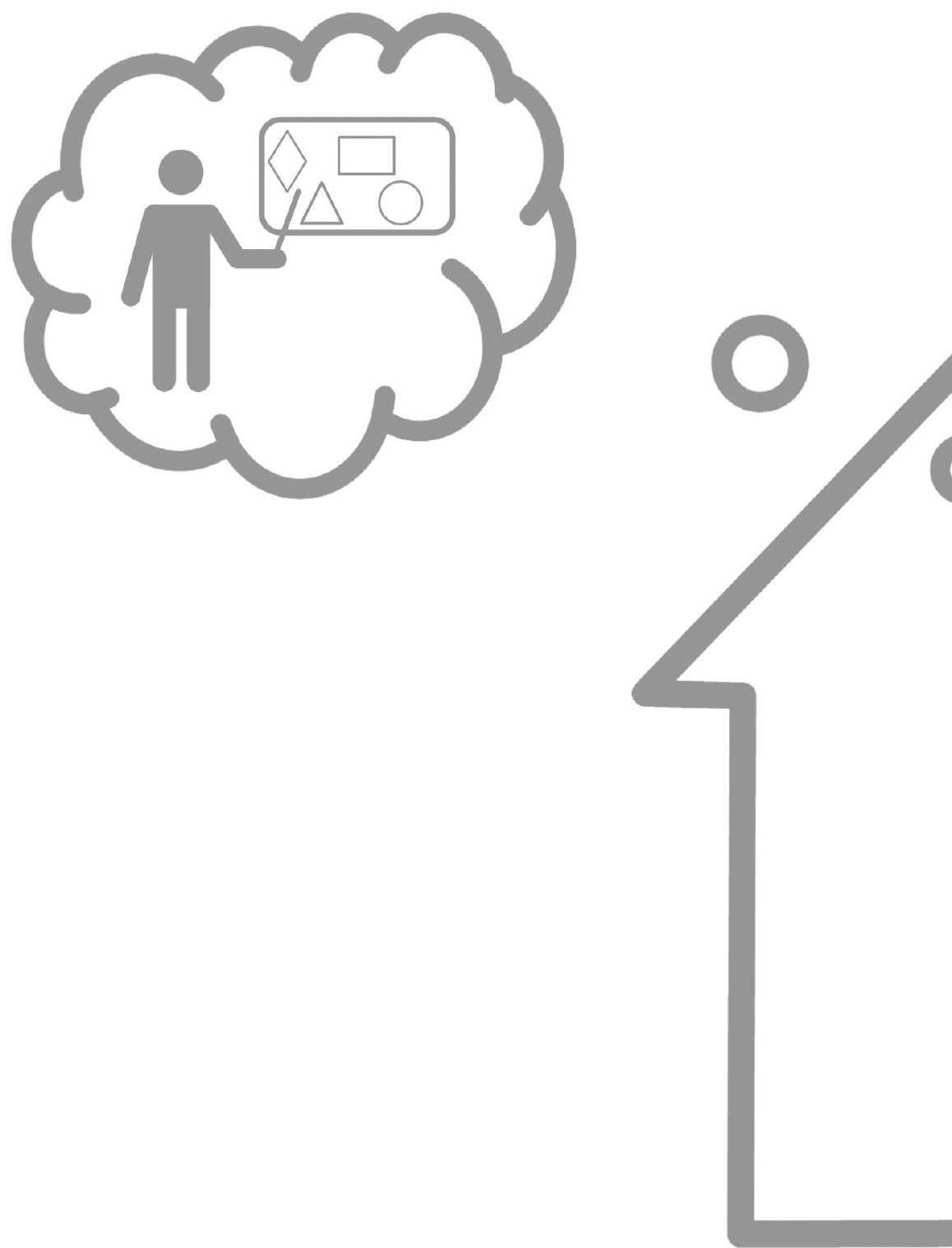


\section{Chapter 2}

\section{Older people's preferences regarding program formats for managing concerns about falls}

programme formats for managing concerns about falls. Age Ageing. 2012, 41(4):474-481. 


\section{Abstract}

Objective To explore the preferences of community-dwelling older persons regarding different program formats for managing concerns about falls.

Subjects and design Cross-sectional study of 5,755 community-dwelling people aged $\geq 70$ years in the Netherlands.

Methods A questionnaire assessed people's willingness to participate per program format $(n=6)$, i.e., a program at home, via telephone, via home visits and telephone consultations, via television or via internet.

Results Of the 2,498 responders, $62.7 \%$ indicated no interest in any of the formats. The willingness to participate per program format varied between 21.5\% (at home) and 9.4\% (via internet). Among people interested in at least one of the formats ( $n=931$ ), higher levels of fall-related concerns were associated with increased preference for a program with home visits. Poor perceived health and age of $\geq 80$ years was associated with less preference for a group program. Higher educated people were more in favor of a program via internet compared to their lower educated counterparts.

Conclusions The majority of community-dwelling older people are not likely to participate in any of the six proposed program formats for managing concerns about falls. However, when diverse formats of effective programs will be made available, uptake and adherence may be increased since program preferences are associated to specific population characteristics. 


\section{Introduction}

Concerns about falls, often labelled as fear of falling, are frequently reported by community-dwelling older people. ${ }^{1}$ Concerns about falls may lead to negative consequences such as activity avoidance ${ }^{2,3}$, physical decline ${ }^{4}$, increased fall risk ${ }^{5,6}$, reduced quality of life $^{7}$, feelings of depression and anxiety ${ }^{8}$ and increased risk of nursing home admission ${ }^{7}$. Certain programs have the ability to reduce concerns about falls, whether they are primarily or not primarily focused on managing concerns about falls. ${ }^{9}$ Nevertheless, among the eligible populations in trials that primarily focused on these concerns, the older people's willingness to take part in (mostly group) programs was rather low. ${ }^{10-14}$ Moreover, drop-out rates of up to $40 \%$ among participants who enrolled in a program are not uncommon. ${ }^{10-17}$ This low uptake and high drop-out rate may be caused by various factors - for example a mismatch between program requirements and participants' capabilities, or between program format and participants' preferences. Clearly, a match between preferences and program format may increase uptake and adherence.

Yardley and colleagues studied older people's preferences regarding three fall prevention activities: 1) professionally supervised strength and balance training in a group; 2) strength and balance training at home following general instructions; and 3) support regarding home modifications. The home-based programs better suited the respondents' preferences: around 60\% indicated their interest for a home-based program versus $40 \%$ for the group-based program. ${ }^{18}$ However, there is hardly any knowledge regarding the willingness of older people to participate in a program aimed at managing concerns about falls and whether this willingness to participate is related to the format in which the program is offered - for instance, via telephone, television or internet.

The aim of this study was to explore the preferences of community-dwelling older people regarding six different formats of a program that primarily focuses on managing concerns about falls. In addition, the associations between format preferences and several demographic, health-related and fall-related characteristics were studied.

\section{Methods}

\section{Study design and participants}

A cross-sectional study was carried out in the south of the Netherlands as part of the screening procedure for an RCT. Between March and December of 2009, a short questionnaire was mailed to 5,755 community-dwelling people aged $\geq 70$ years. People from the general population were randomly selected to participate in the study by local municipal registry offices. A reminder was sent if the questionnaire was not returned within a fortnight. The Medical Ethics Committee of Maastricht University/Academic Hospital Maastricht approved this study. 


\section{Measures}

To assess the willingness of respondents to participate in a program for managing concerns about falls, the following question was formulated:

There are different ways to learn how to manage concerns about falls. A number of possible program formats, which consist of eight sessions each and are supervised by a community nurse, are presented below. Consider that you are concerned about falling and that you would like to do something about this. In which of the following program formats would you be willing to participate: 1) in a group (program with 8 to 10 persons located in your neighborhood); 2) at home; 3) by telephone; 4) at home and by telephone (a combination of format 2 and 3); 5) via television; or 6) via internet?

For each format, people indicated their willingness to participate by answering with 'definitely yes', 'maybe yes', 'maybe no' or 'definitely no'.

Furthermore, concerns about falls ("Are you concerned about falling?") and associated activity avoidance ("Do you avoid certain activities due to concerns about falls?") were assessed. Answer options were: 'never', 'almost never', 'sometimes', 'regularly', 'often' and 'very often'. These answer options were then arranged in categories of 'never' (i.e., never and almost never), 'sometimes', and 'often' (i.e., regularly till very often). In addition, several socio-demographic and health-related characteristics were assessed: age (70-75, 75-79 or $\geq 80$ years), gender (male or female), living situation (alone or not alone), educational level based on completed education and completed professional courses during lifetime (low, middle or high) ${ }^{19}$, perceived general health (good, fair or poor) ${ }^{20}$ and falls in the past six months (never, once or more than once).

\section{Statistical analyses}

Differences in age and gender between responders and non-responders to the questionnaire were tested using a Student's t-test for age and a chi-square test for gender. Descriptive analyses were used to obtain insight into the characteristics and preferences of the participants. Prior to the logistic regression analyses answer options of the dependent variable 'willingness to participate in a specific program format' were dichotomized into 'yes' ('definitely yes' and 'maybe yes') and 'no' ('definitely no' and 'maybe no'). Univariate logistic regression analyses were performed to identify associations between the characteristics and program format preference. Multivariate logistic regression analyses, in which all variables were included, were performed to test which of the characteristics were independently associated with preference for a program format. These analyses were first performed in the total group of responders $(N=2,498)$ and next in the subgroup of responders who indicated their interest for at least one of the program formats ( $n=931$ ). A 0.05 level of significance was used for all analyses. All data were analyzed using SPSS 17.0. 


\section{Results}

Of the 5,755 sent questionnaires, 2,997 were returned (response rate: $52.1 \%$ ). Since 499 questionnaires were incomplete 2,498 were included in the analyses. Nonresponders and those excluded (total $n=3,257$ ) differed significantly from responders $(n=2,498)$ regarding age (mean age 77.6 , standard deviation (SD) $=5.7$ versus mean age 77.0, SD $=5.3$, respectively; $p \leq .001$ ) and gender (60.7 versus $56.6 \%$ female, respectively; $\mathrm{p}=.02$ ).

Table 1 (second column) shows the characteristics of the responders. In short, nearly $45 \%$ were male, nearly $40 \%$ were aged $70-74$ years and more than $50 \%$ rated their general health as good. One or more falls were reported by less than $30 \%$ of the responders, slightly over $45 \%$ reported that they had concerns about falls sometimes till very often, and almost 35\% reported that they avoided activities sometimes till very often due to concerns about falls.

Table 1 also shows that the minority of the responders of the total group (37.3\%) were interested in participating in one of the formats. Among the responders who actually reported concerns about falls, the interest to participate in at least one format increased to $45.8 \%$ (for those who reported having concerns about falls sometimes) and $59.2 \%$ (for those who reported having concerns about falls regularly till very often). There was an overlap in preferences, indicating that some people were willing to participate in multiple program formats. Only $2.4 \%$ would participate in each of the presented formats (not tabulated). Univariate logistic regression analysis showed that older age, status of living alone, higher educational level, poorer perceived general health, more falls in the past 6 months, higher levels of concerns about falls and avoidance of activities were all significantly related to the preference to participate in at least one format (Table 1). Men and women were equally interested in program formats since no association was shown for gender. After controlling for the characteristics (multivariate analysis), the significant associations for higher age and perceived general health disappeared. The preferences of the responders regarding the six different formats for programs for managing concerns about falls are illustrated in Table 2. People were most willing to participate in a program that consists of only home visits, and were least willing to participate in an internet program, 21.5 and 9.4\%, respectively. The preference rates for the other program formats varied between 17 and $19 \%$. 
Table 1 Population characteristics, preferences for formats of a program for managing concerns about falls and univariate and multivariate associations $(\mathrm{N}=2,498)$

\begin{tabular}{|c|c|c|c|c|c|}
\hline \multirow[b]{3}{*}{ Characteristics } & & \multicolumn{2}{|c|}{$\begin{array}{l}\text { Preference for intervention } \\
\text { formats }^{a}\end{array}$} & \multicolumn{2}{|l|}{ Associations } \\
\hline & & \multirow{2}{*}{$\frac{\text { None }}{\mathrm{n}(\%)}$} & \multirow{2}{*}{ At least one } & \multirow{2}{*}{$\begin{array}{l}\text { Univariate } \\
\text { OR }(95 \% \mathrm{Cl})\end{array}$} & \multirow{2}{*}{$\frac{\text { Multivariate }}{\text { OR }(95 \% \mathrm{CI})}$} \\
\hline & & & & & \\
\hline Total population & $2,498(100.0)$ & $1,567(62.7)$ & $931(37.3)$ & & \\
\hline \multicolumn{6}{|l|}{ Demographic } \\
\hline \multicolumn{6}{|l|}{ Gender } \\
\hline Male & $1,085(43.4)$ & $686(63.2)$ & $399(36.8)$ & - & - \\
\hline Female & 1,413 (56.6) & $881(62.3)$ & $532(37.7)$ & $1.04(0.88-1.22)$ & 0.89 (0.73-1.09) \\
\hline \multicolumn{6}{|l|}{ Age } \\
\hline $70-74$ years & $981(39.3)$ & $661(67.4)$ & $320(32.6)$ & - & - \\
\hline $75-79$ years & $784(31.4)$ & $464(59.2)$ & $320(40.8)$ & 1.43 (1.17-1.73) & $1.26(1.02-1.55)$ \\
\hline$\geq 80$ years & $733(29.3)$ & $442(60.3)$ & $291(39.7)$ & $1.36(1.11-1.66)$ & $0.92(0.74-1.16)$ \\
\hline \multicolumn{6}{|l|}{ Living situation } \\
\hline Not alone & 1,481 (59.3) & $969(65.4)$ & $512(34.6)$ & - & - \\
\hline Alone & $1,017(40.7)$ & $598(58.8)$ & $419(41.2)$ & $1.33(1.13-1.56)$ & $1.21(1.00-1.46)$ \\
\hline \multicolumn{6}{|l|}{ Educational level } \\
\hline Low & $1,354(54.2)$ & 947 (69.9) & $407(30.1)$ & - & - \\
\hline Middle & 765 (30.6) & $408(53.3)$ & $357(46.7)$ & $2.04(1.70-2.45)$ & $2.39(1.96-2.92)$ \\
\hline High & $379(15.2)$ & $212(55.9)$ & $167(44.1)$ & $1.83(1.45-2.32)$ & $2.26(1.75-2.91)$ \\
\hline \multicolumn{6}{|l|}{$\underline{\text { Health-related }}$} \\
\hline \multicolumn{6}{|l|}{ Perceived general health } \\
\hline Good & 1,396 (55.9) & $925(66.3)$ & $471(33.7)$ & - & - \\
\hline Fair & 987 (39.5) & $584(59.2)$ & $403(40.8)$ & $1.36(1.15-1.60)$ & $0.92(0.76-1.12)$ \\
\hline Poor & 115 (4.6) & $58(50.4)$ & 57 (49.6) & $1.93(1.32-2.83)$ & $1.07(0.69-1.65)$ \\
\hline \multicolumn{6}{|l|}{ Fall-related } \\
\hline \multicolumn{6}{|l|}{ Falls in the past 6 months } \\
\hline Never & $1,808(72.4)$ & $1,225(67.8)$ & $583(32.2)$ & - & - \\
\hline Once & $408(16.3)$ & $217(53.2)$ & $191(46.8)$ & $1.85(1.49-2.30)$ & $1.38(1.09-1.74)$ \\
\hline More than once & $282(11.3)$ & $125(44.3)$ & $157(55.7)$ & $2.64(2.05-3.41)$ & $1.47(1.10-1.97)$ \\
\hline \multicolumn{6}{|l|}{ Concerns about falls } \\
\hline (Almost) never & $1,346(53.9)$ & $990(73.6)$ & $356(26.4)$ & - & - \\
\hline Sometimes & 797 (31.9) & $432(54.2)$ & $365(45.8)$ & $2.35(1.95-2.83)$ & $2.03(1.63-2.54)$ \\
\hline Regular till very often & $355(14.2)$ & $145(40.8)$ & $210(59.2)$ & $4.03(3.16-5.14)$ & $3.11(2.22-4.35)$ \\
\hline \multicolumn{6}{|l|}{ Avoidance of activities } \\
\hline (Almost) never & $1,648(66.0)$ & $1,152(69.9)$ & $496(30.1)$ & - & - \\
\hline Sometimes & $493(19.7)$ & $249(50.5)$ & $244(49.5)$ & $2.28(1.85-2.80)$ & $1.56(1.22-2.00)$ \\
\hline Regular till very often & $355(14.2)$ & $166(46.5)$ & $191(53.5)$ & $2.67(2.12-3.37)$ & $1.46(1.06-2.03)$ \\
\hline
\end{tabular}

a The dependent variable 'willingness to participate in a program for managing concerns about falls' was dichotomized into preference for program formats of 'none' and 'at least 1'. 'None' implies that respondents answered 'maybe no' or 'definitely no' for all six formats; 'at least one' implies that respondents answered 'definitely yes' or 'maybe yes' for at least one of the six presented formats. $\mathrm{OR}=$ odds ratio; $\mathrm{Cl}=$ confidence interval; [-] = reference group. Odds ratios with significant results are displayed in bold. 
Table 2 Preferences for formats of a program for managing concerns about falls according to population characteristics $(\mathrm{N}=2,498)$

\begin{tabular}{|c|c|c|c|c|c|c|}
\hline \multirow[b]{3}{*}{ Characteristics } & \multicolumn{6}{|c|}{ More likely to participate in an intervention format ${ }^{\mathrm{a}}$ : } \\
\hline & in a group & at home & by telephone & $\begin{array}{l}\text { at home and } \\
\text { by telephone }\end{array}$ & via television & via internet \\
\hline & $\mathrm{n}(\%)$ & $\mathrm{n}(\%)$ & $\mathrm{n}(\%)$ & $\mathrm{n}(\%)$ & $\mathrm{n}(\%)$ & $\mathrm{n}(\%)$ \\
\hline Total population & $429(17.2)$ & $536(21.5)$ & $441(17.7)$ & $468(18.7)$ & $438(17.5)$ & $234(9.4)$ \\
\hline \multicolumn{7}{|l|}{ Demographic } \\
\hline \multicolumn{7}{|l|}{ Gender } \\
\hline Male & $183(16.9)$ & $236(21.8)$ & $189(17.4)$ & $208(19.2)$ & $197(18.2)$ & $147(13.5)$ \\
\hline Female & $246(17.4)$ & $300(21.2)$ & $252(17.8)$ & $260(18.4)$ & $241(17.1)$ & $87(6.2)$ \\
\hline \multicolumn{7}{|l|}{ Age } \\
\hline $70-74$ years & $161(16.4)$ & $174(17.7)$ & $158(16.1)$ & $150(15.3)$ & $161(16.4)$ & 117 (11.9) \\
\hline 75-79 years & $154(19.6)$ & $176(22.4)$ & $158(20.2)$ & $164(20.9)$ & $156(19.9)$ & $80(10.2)$ \\
\hline$\geq 80$ years & $114(15.6)$ & $186(25.4)$ & $125(17.1)$ & $154(21.0)$ & $121(16.5)$ & $37(5.0)$ \\
\hline \multicolumn{7}{|l|}{ Living situation } \\
\hline Not alone & $230(15.5)$ & $283(19.1)$ & $243(16.4)$ & $244(16.5)$ & $248(16.7)$ & $159(10.7)$ \\
\hline Alone & $199(19.6)$ & $253(24.9)$ & $198(19.5)$ & $224(22.0)$ & $190(18.7)$ & $75(7.4)$ \\
\hline \multicolumn{7}{|l|}{ Educational level } \\
\hline Low & $173(12.8)$ & $239(17.7)$ & $183(13.5)$ & $204(15.1)$ & $176(13.0)$ & $60(4.4)$ \\
\hline Middle & $182(23.8)$ & $205(26.8)$ & $165(21.6)$ & $172(22.5)$ & $171(22.4)$ & $103(13.5)$ \\
\hline High & 74 (19.5) & $92(24.3)$ & $93(24.5)$ & $92(24.3)$ & $91(24.0)$ & 71 (18.7) \\
\hline \multicolumn{7}{|l|}{ Health-related } \\
\hline \multicolumn{7}{|l|}{$\begin{array}{l}\text { Perceived general } \\
\text { health }\end{array}$} \\
\hline Good & $226(16.1)$ & $258(18.5)$ & $235(16.8)$ & $223(16.0)$ & $239(17.1)$ & $149(10.7)$ \\
\hline Fair & $186(18.8)$ & $236(23.9)$ & $183(18.5)$ & $210(21.3)$ & $178(18.0)$ & $77(7.8)$ \\
\hline Poor & 17 (14.8) & $42(36.5)$ & $23(20.0)$ & $35(30.4)$ & 21 (18.3) & $8(7.0)$ \\
\hline \multicolumn{7}{|l|}{ Fall-related } \\
\hline \multirow{2}{*}{\multicolumn{7}{|c|}{$\begin{array}{l}\text { Falls in the past } 6 \\
\text { months }\end{array}$}} \\
\hline & & & & & & \\
\hline Never & $268(14.8)$ & $315(17.4)$ & $275(15.2)$ & $271(15.0)$ & $282(15.6)$ & 161 (8.9) \\
\hline Once & $87(21.3)$ & $115(28.2)$ & $95(23.3)$ & $102(25.0)$ & $92(22.5)$ & $42(10.3)$ \\
\hline More than once & $74(26.2)$ & $106(37.6)$ & $71(25.2)$ & $95(33.7)$ & $64(22.7)$ & 31 (11.0) \\
\hline \multicolumn{7}{|l|}{ Concerns about falls } \\
\hline (Almost) never & $162(12.0)$ & $170(12.6)$ & $162(12.0)$ & $153(11.4)$ & $185(13.7)$ & $123(9.1)$ \\
\hline Sometimes & $169(21.2)$ & $219(27.5)$ & $172(21.6)$ & $187(23.5)$ & $162(20.3)$ & $75(9.4)$ \\
\hline Regular till very often & $98(27.6)$ & $147(41.4)$ & $107(30.1)$ & $128(36.1)$ & $91(25.6)$ & $36(10.1)$ \\
\hline \multicolumn{7}{|l|}{ Avoidance of activities } \\
\hline (Almost) never & $234(14.2)$ & $254(15.4)$ & $231(14.0)$ & $223(13.5)$ & $242(14.7)$ & $150(9.1)$ \\
\hline Sometimes & $119(24.1)$ & $152(30.8)$ & $122(24.7)$ & $131(26.6)$ & $123(24.9)$ & $53(10.8)$ \\
\hline Regular till very often & $76(21.3)$ & $130(36.4)$ & $88(17.7)$ & $114(31.9)$ & $73(20.4)$ & $31(8.7)$ \\
\hline
\end{tabular}

a The dependent variable 'willingness to participate in a program for managing concerns about falls' was dichotomized into 'yes' and 'no'. More likely to participate implies that respondents answered 'definitely yes' or 'maybe yes' to the format compared to 'definitely no' or 'maybe no'. 
Table 3 illustrates the univariate associations between the preferences of responders who were willing to participate in at least one program format $(n=931)$ and the demographic, health-related and fall-related characteristics. For example, people $\geq 80$ years and those with poor perceived general health were less in favor of the group program than the younger age groups and those with good perceived general health. In contrast, a program with only home visits was preferred by the oldest people as well as the people with poor perceived general health. The same holds for those who reported multiple falls, concerns about falls and avoidance of activities. See Table 3 for all univariate associations.

Although many associations disappeared after controlling for the other characteristics in the multivariate analyses, several significant associations were observed (see Appendix, Supplementary data are available in Age and Ageing online). The oldest people and those with a poor perceived general health, had significantly less preference for a group program. The likelihood of participating in a program with home visits only was associated with higher levels of concerns about falls; also, in comparison to men women did not prefer this format. The univariate associations for age and perceived general health disappeared. A program by telephone was associated only with a high educational level. In comparison to the program with home visits only, women were less willing to participate in a program with a combination of home visits and telephone consultations; however, people with concerns about falls preferred this format. No associations were found between any of the characteristics and the television program format. Being a woman, being older and having a low level of education were all associated with less preference for a program via internet.

\section{Discussion}

This cross-sectional study among community-dwelling older people showed that nearly $63 \%$ of respondents expressed no interest in participating in any of the six proposed program formats for managing concerns about falls, despite considering the presence of these concerns in their lives. This percentage decreased to $41 \%$, however, for those actually reporting (either regularly, often or very often) concerns about falls. The program at home was preferred the most (about 22\%) and the program via the internet least (about 9\%). The preference rates for the other formats hardly varied, i.e., they ranged between $17 \%$ for the group-based program and $19 \%$ for the program with a combination of home visits and telephone consultations. The likelihood to participate in at least one program format was independently associated with being 74-79 years of age, living alone, higher educational level, having more falls in the past 6 months, having higher levels of concerns about falls and avoidance of activities. For the subgroup of people who were willing to participate in at least one program, higher levels of concerns about falls were independently associated with the preference for a program that in- 
cludes home visits. Furthermore, higher educational level was related to the preference for a program via internet.

In the present study, the preference to participate in the different program formats was generally lower than what Yardley and colleagues reported with respect to their fall prevention programs (between 9 and 22\% versus 40 and $60 \%$, respectively) ${ }^{18}$, but the pattern of a stronger preference for a program at home compared with that in a group setting is similar in both studies.

Several background characteristics, such as gender and level of concerns about falls of those who were willing to participate, are associated with the preference for specific program formats; therefore, it is likely that uptake can be increased by offering different program formats according to these characteristics. The successful cognitive behavioral group program 'A Matter of Balance ${ }^{14,17}$, which addresses concerns about falls and activity avoidance could be made suitable for different formats. ${ }^{21}$ In this perspective the oldest old or those with a poor perceived health could be offered a home-based program and the higher educated people could be offered a format via television or through the internet. Future research is needed to confirm whether or not these program formats are as effective as the above-mentioned group approach wherein also costs should be taken into account.

A few aspects in this study are noteworthy. To the best of our knowledge this was the first study that examined older people's preferences regarding program formats regarding the management of concerns about falls. The question of being concerned about falling and also being willing to do something about these concerns, while indicating a preference for program formats, was specifically designed for this study. Aside from face validity, no additional testing for psychometric properties had been performed. It may have been difficult for people who were not concerned about falling to nevertheless regard themselves as having concern for the subject; however, special interest was given to the preferences of the group that did have concerns about falls. Additionally, it is conceivable that not only preference for a particular format but also the preparedness or motivation to participate in a program more generally was measured. In addition to this study, it would be interesting to ask why people do or do not want to participate in any of the proposed formats, for example with focus group interviews. ${ }^{22,23}$ This may provide information as to in what kind of program format older people with concerns about falls would be willing to participate, as perhaps they are only interested in written information that can be sent to them, or might prefer the program to be led by someone else - a general practitioner or a psychologist. Not all preferences can be responded to in daily practice, however, due to a lack of proven effectiveness of the method or program, or restrictions in resources like money or manpower, or both. Lastly, stating one's intention or willingness to participate in a program format may not reflect actual participation in a program, as can be seen by the 


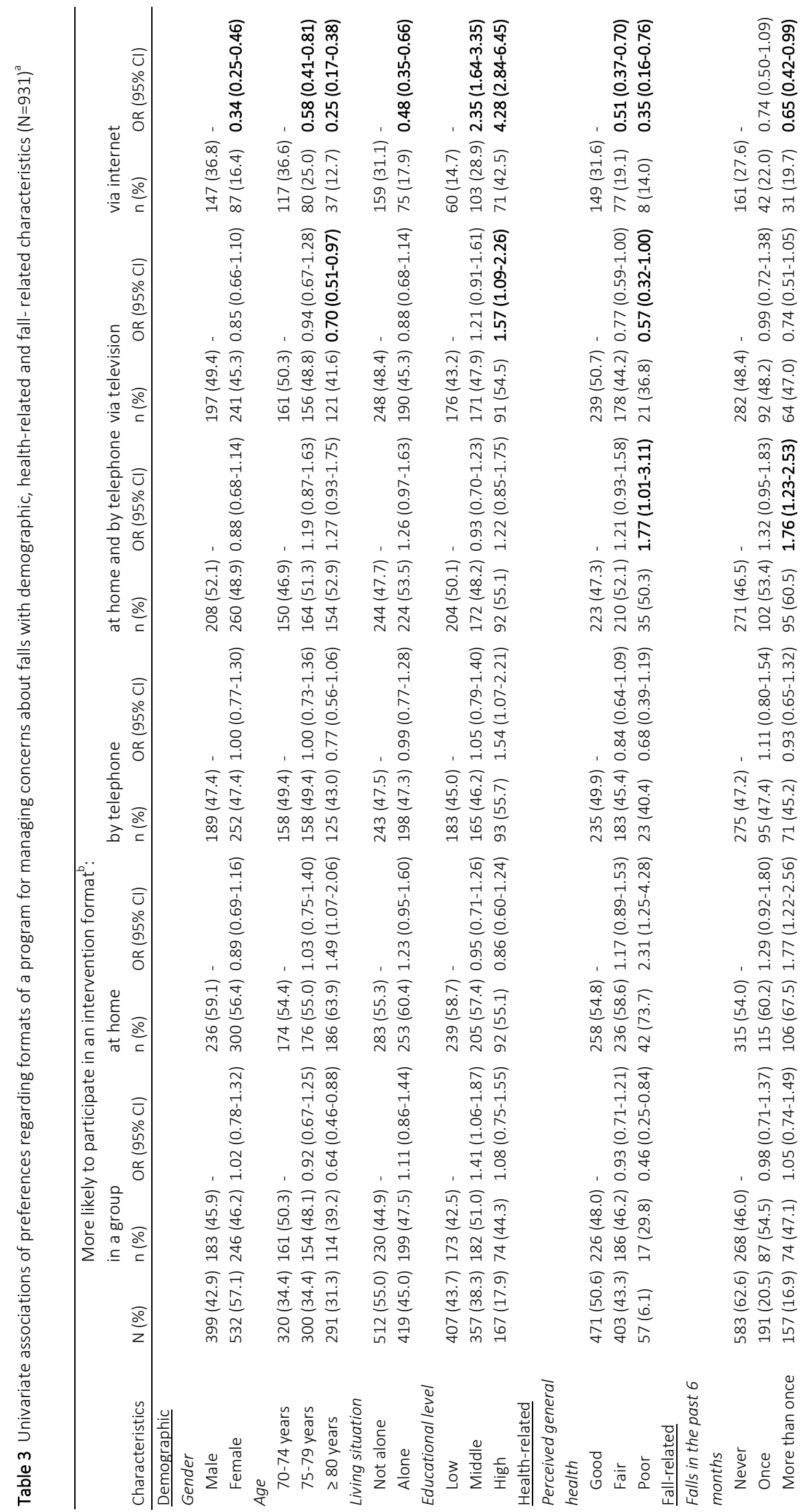




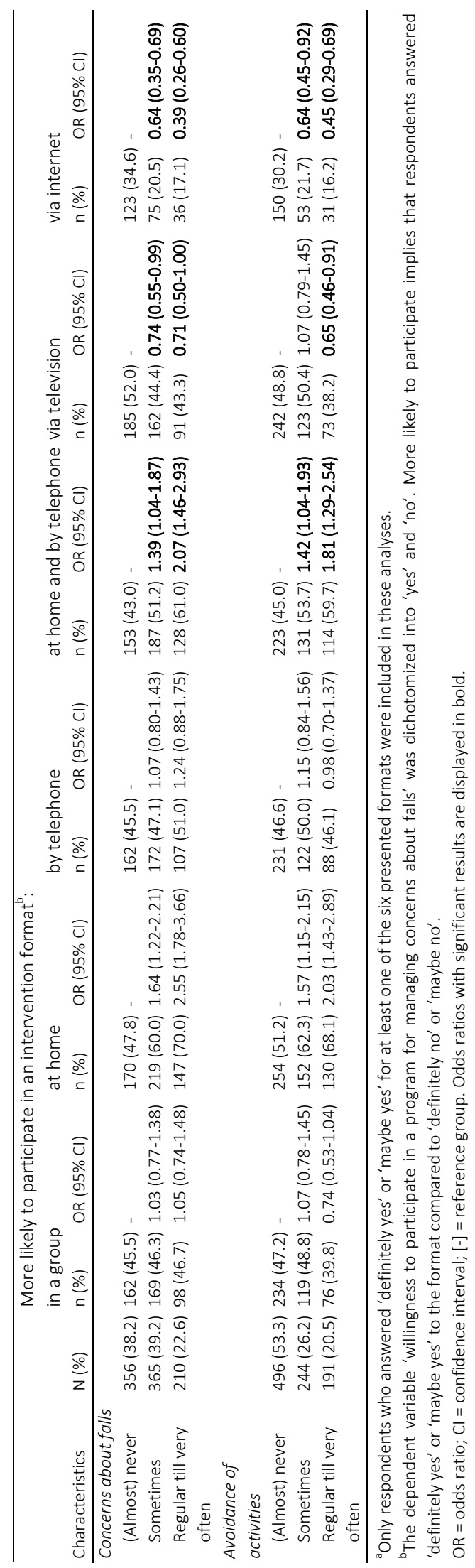


high drop-out rates of such programs. ${ }^{11,12,14,15,17,24}$ In particular, people who have high concerns about falls could experience a substantial tension with regard to participating in any program in which they will be confronted with their concerns. An appropriate recruitment strategy is therefore needed - for example, a face-to-face meeting or a telephone call with the facilitator, in which a potential participant obtains information about the program and its relevant content. ${ }^{25,26}$

Strengths of this study were, however, its largely representative sample of the general population and its accurate comparison of preferences regarding the program format. This was particularly relevant because the information provided about the program (i.e. managing concerns about falls in eight sessions guided by a community nurse) was similar across the different formats. Moreover, our findings are important for practice, as uptake and adherence can be increased by offering older people a program format depending on their characteristics or preferences.

We found that the majority of older people did not want to participate in any of the presented program formats for managing concerns about falls, despite considering the presence of these concerns in their lives, as well as the motivation to do something about these concerns. Yet, it is promising that people who actually reported concerns about falls were substantially more willing to participate. Furthermore, several background characteristics were clearly associated with preferences for specific program formats, thus providing opportunities for tailor-made interventions. We therefore recommend the development of different formats of effective programs for managing concerns about falls. As a result, uptake and adherence of these programs may improve as community-living older people can choose a program that fits their preference.

\section{Key points}

- The majority of community-dwelling older people are not likely to participate in any of the presented program formats to learn to manage concerns about falls, despite their concerns and their desire to do something about it.

- A program format with eight home visits focusing on managing concerns about falls is most popular compared with a program format conducted in a group, via telephone, via a combination of home visits and telephone consultations, via television or via internet.

- Background characteristics, such as gender, perceived general health, level of education and concerns about falls are associated with preferences for specific program formats; therefore acceptance for starting such a program could be increased by offering tailor-made programs according to these characteristics. 


\section{Acknowledgements}

We would like to thank the municipal registry offices of Maastricht, Heerlen and SittardGeleen and the Centre for Data and Information Management (MEMIC) for their assistance in this study. This research was conducted as part of a randomized controlled trial that has been funded by ZonMw - the Netherlands Organization for Health Research and Development (grant 120610001). Participation of one of the authors was supported by the Odysseus Grant "The Psychology of Pain and Disability Research Program" funded by the Research Foundation - Flanders, Belgium (FWO Vlaanderen). 


\section{References}

1. Scheffer AC, Schuurmans MJ, van Dijk N, van der Hooft T, de Rooij SE: Fear of falling: measurement strategy, prevalence, risk factors and consequences among older persons. Age Ageing. 2008, 37(1):19-24.

2. Tinetti ME, Powell L: Fear of falling and low self-efficacy - a cause of dependence in elderly persons. $J$ Gerontol. 1993, 48:35-38.

3. Deshpande N, Metter EJ, Lauretani F, Bandinelli S, Guralnik J, Ferrucci L: Activity restriction induced by fear of falling and objective and subjective measures of physical function: a prospective cohort study. $J$ Am Geriatr Soc. 2008, 56(4):615-620.

4. Delbaere K, Crombez G, Vanderstraeten G, Willems T, Cambier D: Fear-related avoidance of activities, falls and physical frailty. A prospective community-based cohort study. Age Ageing. 2004, 33(4):368-373.

5. Delbaere K, Close JC, Brodaty H, Sachdev P, Lord SR: Determinants of disparities between perceived and physiological risk of falling among elderly people: cohort study. BMJ. 2010, 341:c4165.

6. Friedman SM, Munoz B, West SK, Rubin GS, Fried LP: Falls and fear of falling: which comes first? A longitudinal prediction model suggests strategies for primary and secondary prevention. J Am Geriatr Soc. 2002, 50(8):1329-1335.

7. Cumming RG, Salkeld G, Thomas M, Szonyi G: Prospective study of the impact of fear of falling on activities of daily living, SF-36 scores, and nursing home admission. J Gerontol A Biol Sci Med Sci. 2000, 55(5):M299-305.

8. Arfken CL, Lach HW, Birge SJ, Miller JP: The prevalence and correlates of fear of falling in elderly persons living in the community. Am J Public Health. 1994, 84(4):565-570.

9. Bula CJ, Monod S, Hoskovec C, Rochat S: interventions aiming at balance confidence improvement in older adults: an updated review. Gerontology. 2011, 57(3):276-286.

10. Brouwer BJ, Walker C, RydahI SJ, Culham EG: Reducing fear of falling in seniors through education and activity programs: a randomized trial. J Am Geriatr Soc. 2003, 51(6):829-834.

11. Clemson L, Cumming RG, Kendig H, Swann M, Heard R, Taylor K: The effectiveness of a communitybased program for reducing the incidence of falls in the elderly: a randomized trial. J Am Geriatr Soc. 2004, 52(9):1487-1494.

12. Sattin RW, Easley KA, Wolf SL, Chen Y, Kutner MH: Reduction in fear of falling through intense tai chi exercise training in older, transitionally frail adults. J Am Geriatr Soc. 2005, 53(7):1168-1178.

13. Zhang JG, Ishikawa-Takata K, Yamazaki H, Morita T, Ohta T: The effects of Tai Chi Chuan on physiological function and fear of falling in the less robust elderly: an intervention study for preventing falls. Arch Gerontol Geriatr. 2006, 42(2):107-116.

14. Zijlstra GA, van Haastregt JC, Ambergen T, van Rossum E, van Eijk JT, Tennstedt SL, Kempen GI: Effects of a multicomponent cognitive behavioral group intervention on fear of falling and activity avoidance in community-dwelling older adults: results of a randomized controlled trial. J Am Geriatr Soc. 2009, 57(11):2020-2028.

15. Arai T, Obuchi S, Inaba Y, Nagasawa H, Shiba Y, Watanabe S, Kimura K, Kojima M: The effects of shortterm exercise intervention on falls self-efficacy and the relationship between changes in physical function and falls self-efficacy in Japanese older people: a randomized controlled trial. Am J Phys Med Rehabil. 2007, 86(2):133-141.

16. Liu H, Rainey J, Zabel R, Quiben MU, Kehayov A, Boswell JK: Comparison of two exercise programs using the Falls Efficacy Scale, Berg Balance Scale and ankle dorsiflexor strength in older adults. Phys Occup Ther Geriatr. 2007(26):23-42.

17. Tennstedt S, Howland J, Lachman M, Peterson E, Kasten L, Jette A: A randomized, controlled trial of a group intervention to reduce fear of falling and associated activity restriction in older adults. J Gerontol B Psychol Sci Soc Sci. 1998, 53(6):P384-392.

18. Yardley L, Kirby S, Ben-Shlomo Y, Gilbert R, Whitehead S, Todd C: How likely are older people to take up different falls prevention activities? Prev Med. 2008, 47(5):554-558.

19. CBS: Standaard Onderwijsindeling 2006. Editie 2009/'10. Voorburg/Heerlen: Centraal Bureau voor de Statistiek, The Netherlands; 2009. 
20. Stewart AL, Hays RD, Ware JE, Jr.: The MOS short-form general health survey. Reliability and validity in a patient population. Med Care. 1988, 26(7):724-735.

21. Zijlstra GAR: Managing concerns about falls fear of falling and avoidance of activity in older people [PhD Thesis]. Maastricht: Faculty of Health, Medicine and Life Sciences, University Maastricht; 2007.

22. Webb TL, Sheeran P: Does changing behavioral intentions engender behavior change? A meta-analysis of the experimental evidence. Psychol Bull. 2006, 132(2):249-268. 


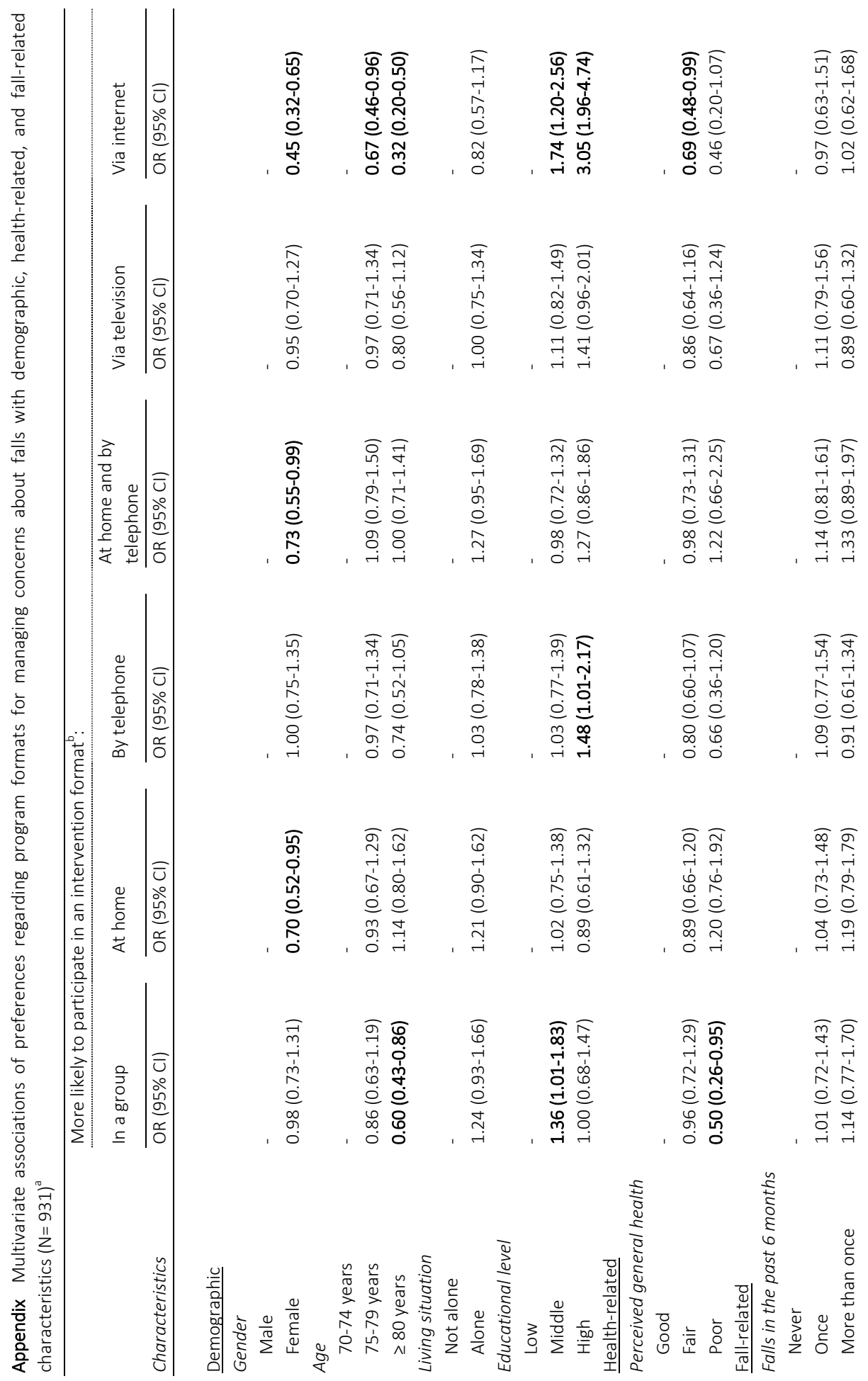




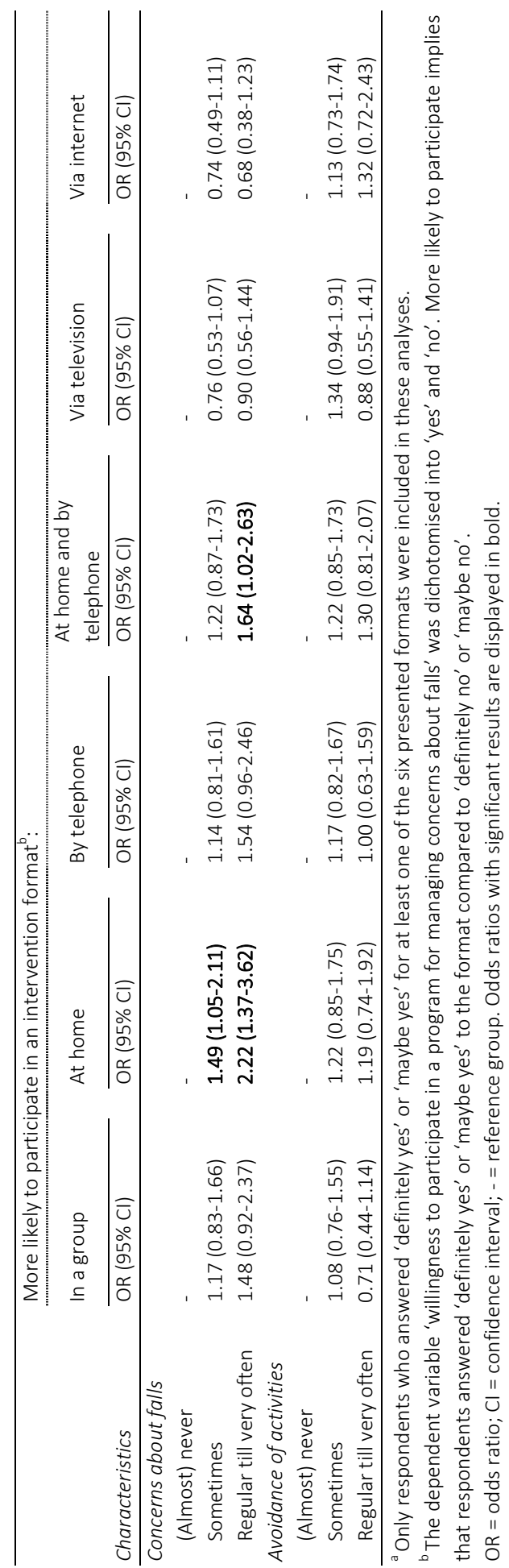



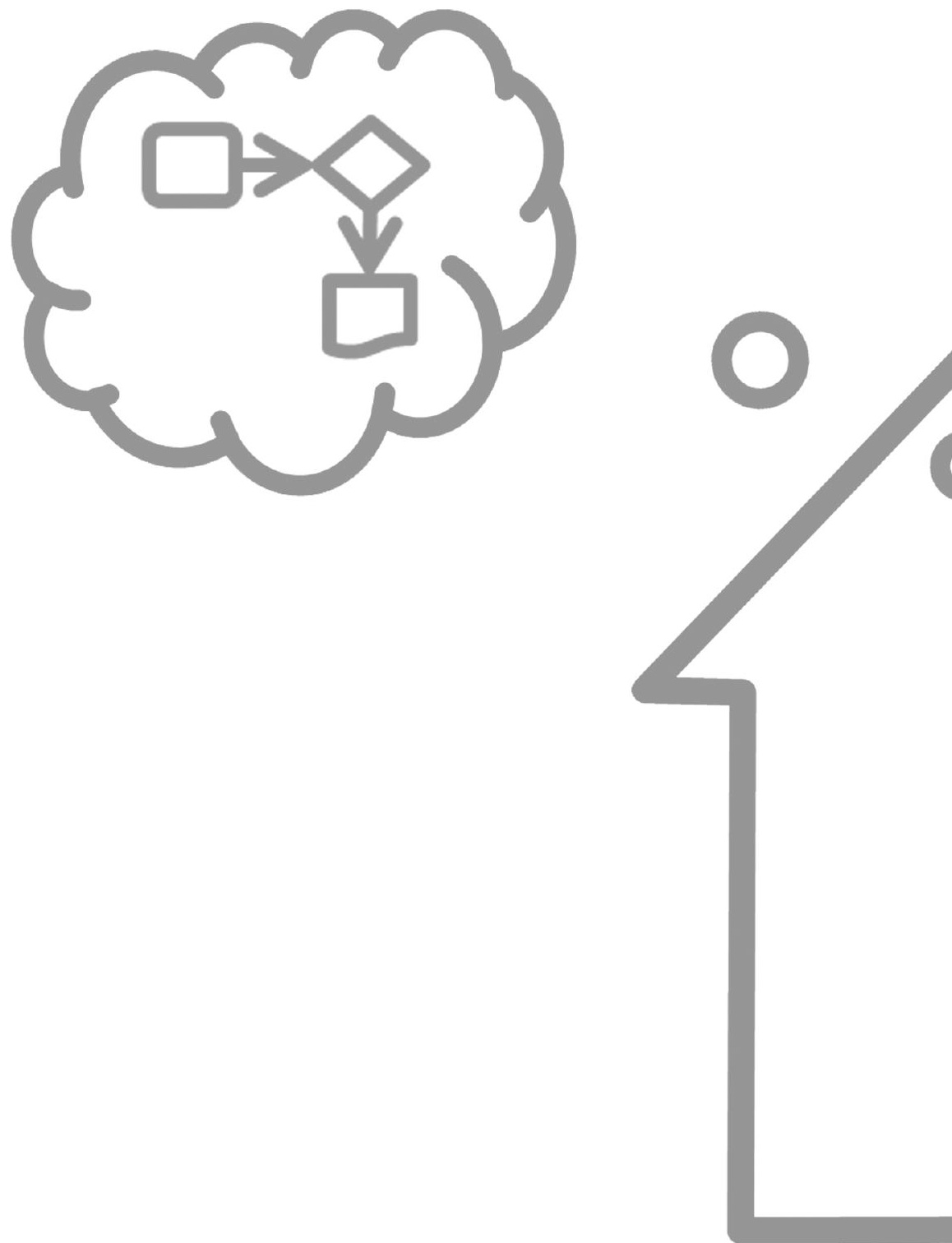


\section{Chapter 3}

\section{Evaluating an in-home multicomponent cognitive behavioral program to manage concerns about falls and associated activity avoidance in frail community-dwelling older people: design of a randomized controlled trial}




\begin{abstract}
Background Concerns about falls are frequently reported by older people. These concerns can have serious consequences such as an increased risk of falls and the subsequent avoidance of activities. Previous studies have shown the effectiveness of a multicomponent group program to reduce concerns about falls. However, owing to health problems older people may not be able to attend a group program. Therefore, we adapted the group approach to an individual in-home program.
\end{abstract}

Methods/Design A two-group randomized controlled trial has been developed to evaluate the in-home multicomponent cognitive behavioral program to manage concerns about falls and associated activity avoidance in frail older people living in the community. Persons were eligible for study if they were 70 years of age or over, perceived their general health as fair or poor, had at least some concerns about falls and associated avoidance of activity. After screening for eligibility in a random sample of older people, eligible persons received a baseline assessment and were subsequently allocated to the intervention or control group. Persons assigned to the intervention group were invited to participate in the program, while those assigned to the control group received care as usual. The program consists of seven sessions, comprising three home visits and four telephone contacts. The sessions are aimed at instilling adaptive and realistic views about falls, as well as increasing activity and safe behavior. An effect evaluation, a process evaluation and an economic evaluation are conducted. Follow-up measurements for the effect evaluation are carried out 5 and 12 months after the baseline measurement. The primary outcomes of the effect evaluation are concerns about falls and avoidance of activity as a result of these concerns. Other outcomes are disability and falls. The process evaluation measures: the population characteristics reached; protocol adherence by facilitators; protocol adherence by participants (engagement in exposure and homework); opinions about the program of participants and facilitators; perceived benefits and achievements; and experienced barriers. The economic evaluation examines the impact on health-care utilization, as well as related costs.

Discussion/Progress A total number of 389 participants is included in the study. Final results are expected in 2012.

Trial registration NCT01358032 


\section{Background}

Falls and concerns related to falls are very common in community-dwelling older people. About one in three older people living in the community experiences at least one fall each year, of which roughly half results in an injury. ${ }^{1,2}$ Interestingly, around twothirds of older people report concerns related to falls ${ }^{3}$, of which roughly half report activity avoidance as a result of these concerns. ${ }^{4-8}$ Concerns about falls can be present in both people who have fallen and people who have not. ${ }^{9}$ These concerns have been linked to decreased balance performance, decreased mobility, functional decline, low quality of life, institutionalization and falls. ${ }^{3,10-13}$ Recent research has shown that concerns about falls can lead to falls irrespective of any physiological fall risk. ${ }^{11}$

Concerns about falls can be considered as a multifactorial problem. ${ }^{14,15}$ As a consequence, successful programs should not only target concerns about falls but should also focus on aspects like increasing self-efficacy and a sense of control regarding the risks of falling, setting realistic goals for increasing activity, changing the environment to reduce the fall risk and promoting physical activity to increase strength and balance. ${ }^{15-19}$

Cognitive behavioral therapy could be seen as a suitable strategy to reduce concerns about falls by modifying patterns of thoughts (cognition) and actions (behavior) that contribute to the concern.

One of the programs with proven effectiveness is the Dutch version of 'A Matter of Balance' (AMB-NL). ${ }^{20}$ This multicomponent cognitive behavioral group program consists of eight weekly group sessions and a booster session after six months. It has shown favorable effects on concerns about falls, perceived control over falling and daily activity, after at least 8 months of follow-up. In addition, significantly fewer recurrent fallers were observed in this group after 14 months of follow-up. ${ }^{15}$ Notwithstanding these positive outcomes, approximately $40 \%$ of the participants attended less than five sessions out of a total eight, mainly because of health problems. ${ }^{21}$ It seems that, in particular frail older people refrained from attending the group program. This is also seen in other group programs that explicitly address concerns about falls, and target frail older people living in the community. ${ }^{18,22}$

The aim of this project is to develop an in-home program to enable frail older people to participate, as well as people who prefer an in-home approach rather than a group approach. ${ }^{23}$ This paper presents the design of a randomized controlled trial evaluating 'A Matter of Balance at Home' (AMB-Home) in frail older people, living in the community in the Netherlands. The objectives of this trial are to conduct: (1) an effect evaluation to determine the effects of this in-home program on concerns about falls and fall-related activity avoidance, and additional outcomes including disability and fall incidents; (2) a process evaluation to determine the feasibility of the program; and (3) an economic evaluation uncovering the impact of the program on health-care utilization and related costs. 


\section{Methods/Design}

\section{Design}

The study concerns a two-group randomized controlled trial with a baseline measurement and follow-up measurements after 5 (directly after the program) and 12 months (see Figure 1). The selection of potential participants was performed between March and December 2009 in four consecutive cycles. Each cycle lasted about 15 months and included: screening for eligible participants; baseline measurement; stratified randomization; the intervention period; and follow-up measurements. The Medical Ethics Committee of the Maastricht University/Academic Hospital Maastricht in the Netherlands approved this trial.

\section{Recruitment of participants}

Three communities, Maastricht, Sittard-Geleen, and Heerlen, situated in the southeast of the Netherlands have been selected for participation in the trial. The municipal registry offices selected 11,490 addresses of community-dwelling people of 70 years or over in their district, at random. To screen for eligibility, people received a short postal questionnaire with a freepost envelope, as well as information about the trial and an informed consent form. After a fortnight, reminder letters were sent. The questionnaire assessed socio-demographics and fall-related variables and inclusion and exclusion criteria.

Older people were included if they met all of the following criteria: 1 ) they reported at least some concerns about falls; 2) they reported at least some associated avoidance of activity; 3) they perceived their general health as fair or poor ${ }^{24}$; 4 ) they lived in the community; 5) they were 70 years of age or older; and 6) they were willing to participate (signed informed consent form). People were excluded if they were confined to bed, were restricted by the permanent use of a wheelchair, were waiting for a nursing home admission, experienced substantial hearing or vision impairments or they failed the shortened version of the Abbreviated Mental Test (AMT4) ${ }^{25}$ assesses cognitive impairment and, subsequently, the Telephone Interview Cognitive Status (TICS) ${ }^{26}$. In addition, a restriction was applied to couples; to prevent reciprocal influencing only one partner of a couple was allowed to participate in the trial. Lots were drawn if this selection process was necessary.

\section{Randomization}

Participants were assigned to the program or the no-treatment control group directly after the baseline measurement using stratified randomization. This randomization was performed in blocks of two on the basis of one prognostic factor: the level of concern about falls (some, regular, often, and very often). Computerized alternative allocation was used in the randomization process, which was performed by an external agency. 
1a

Continuous registration of falls and health-care utilization

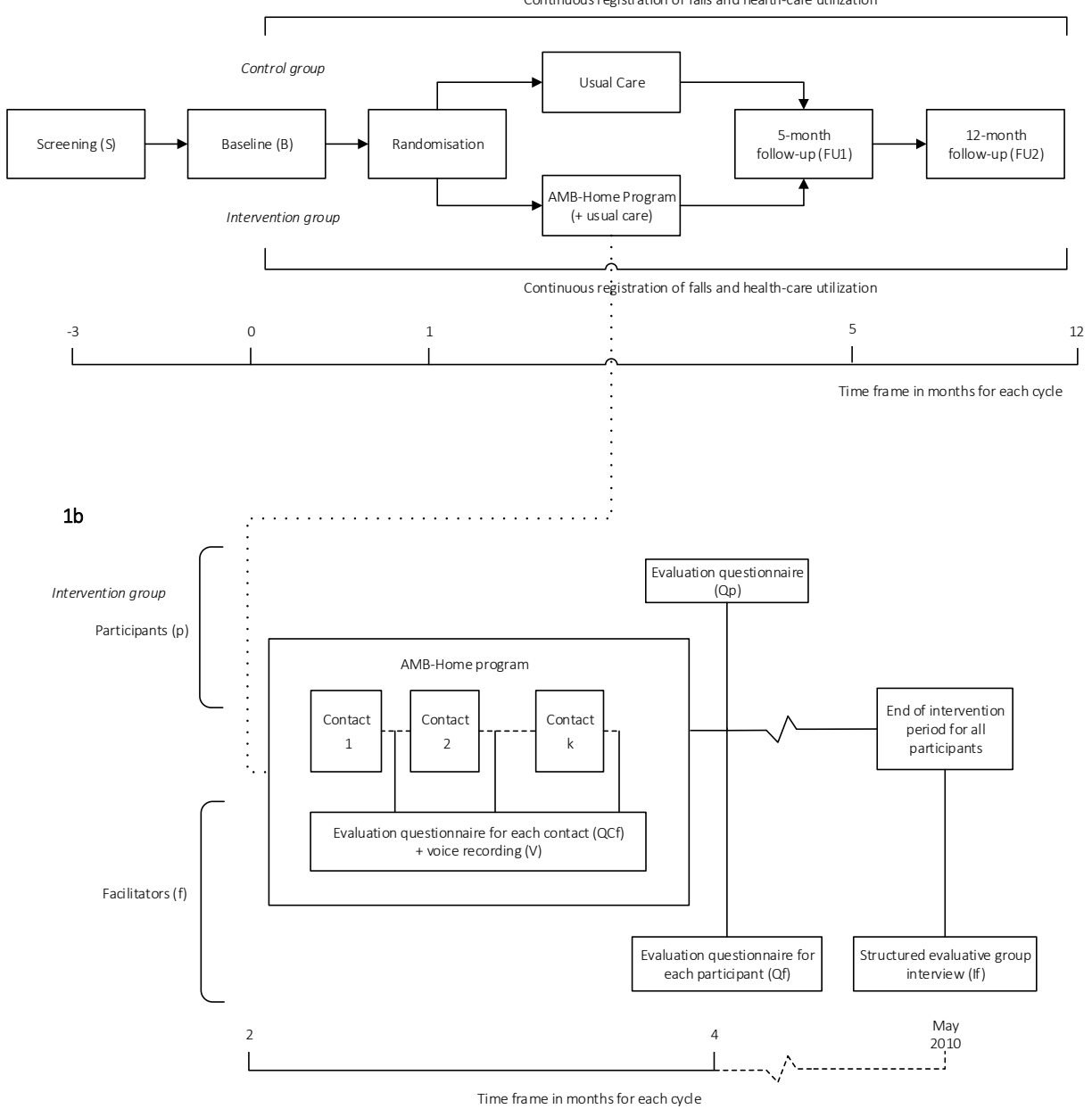

Figure 1 Study design

1a Displaying effect and economic evaluation

Legends: $\mathrm{S}=$ screening; $\mathrm{B}$ = baseline; FU1 = 5-month follow-up; FU2 = 12-month follow-up

$1 \mathrm{~b}$ Displaying process evaluation

Legends: QCf = questionnaire filled in by facilitator for each participant for each contact, $V=$ voice recording for selection of contacts, $\mathrm{Qf}=$ questionnaire filled in by facilitator for each participant, $\mathrm{Qp}=$ questionnaire filled in by participant after program, and If = group interview with facilitators

\section{Program}

\section{Development of the program}

AMB-Home is based on the Dutch version of a cognitive behavioral group program for older people living in the community named 'A Matter of Balance' (AMB-NL). AMB-NL is a translated, adapted and evaluated version of 'A Matter of Balance' ${ }^{20}$, a program that originated in the USA ${ }^{18}$. In the development process of the home-based version for frail 
older people, all components and techniques of the group program were assessed on their appropriateness for the new targeted population, the in-home setting, and future implementation in Dutch health-care. Therefore, experts ( $n=8$, see Acknowledgements) in the domain of program development and behavioral change were consulted and a pilot study with six volunteers was conducted to test the program initial feasibility.

Substantial elements of the AMB-NL program, such as cognitive restructuring, a DVD for modelling purposes, i.e. presenting stories of other people reporting concerns about falls and their solutions, and the discussions on themes related to fall prevention themes are maintained in AMB-Home. However, several adaptations have been made. First, the program of AMB-Home consists of seven sessions (three home visits and four telephone contacts) in contrast to the eight sessions and a booster session after six months in AMB-NL. Second, the formulation and review of personal action plans, regarding the themes of the sessions, receive a more prominent role throughout the program to encourage active participation. ${ }^{21,27}$ Third, the physical exercises performed in AMB-NL are not included in AMB-Home since supervision of the appropriate execution of these exercises is not feasible. Fourth, a new component is added to home visit 3. During this visit, the participant actually practices a specific (fear-related) problem in daily life in the presence of the program facilitator. Lastly, motivational interviewing is added as a discussion technique, to motivate participants to change their beliefs and behavior, regarding concerns about falls and activity avoidance. See Table 1 for additional information on the similarities and differences of both programs.

\section{Contents and format of the program}

The in-home program aims to teach participants how to deal with their concerns about falls and related avoidance of activity, in order to increase their physical, social and functional activities. AMB-Home consists of seven individual sessions, including three home visits (60, 60 and 75 minutes, respectively) and four telephone contacts (35 minutes each). During each session a main theme is addressed. The themes of the program are: concerns about falls; thoughts about falling; physical exercise; asserting oneself; overcoming personal barriers; safe behavior; and managing concerns about falls (see Table 1). All sessions have a similar structure: review the previous session (except the first session); discuss the current session's theme; and formulate a concrete and personal action plan, related to the theme discussed. The contents of each session are described in detail in a facilitator's manual, and participants receive printed materials, including background information on the session's theme and worksheets to complete during or between the sessions.

The principles of cognitive restructuring ${ }^{28}$ are used for shifting maladaptive to adaptive attitudes with respect to falling, as well as for increasing self-efficacy beliefs and feelings of control. Four strategies are applied to obtain these goals: (1) restructuring misconceptions to promote a realistic view of fall-risk and making concerns about falls controllable; (2) setting realistic goals for increasing activity and safe behavior; (3) 
adapting the environment to reduce the fall-risk; and (4) promoting the uptake of daily life activities that are avoided owing to concerns about falls. The following techniques are applied in the program. Motivational interviewing is used as a discussion technique to encourage the internal motivation and increase the self-efficacy of participants. This technique is a client-centered approach, with reflective listening and positive affirmations rather than direct questioning, persuasion, or advice-giving. ${ }^{29}$ In addition, to tailor the program to the participant's needs and preferences, participants are encouraged to come up with activities that they consider important and which they would like to perform safely. These activities are then incorporated into program elements such as action planning. Participants experiencing difficulties in recognizing such activities are prompted in this process by being shown them 16 drawings of activities of the Iconographical Falls Efficacy Scale (Icon-FES). ${ }^{30}$ Action plans are used to bridge the gap between behavioral intentions and behavior itself. In every session a personally relevant activity is chosen by the participant. The 'when', 'where', and 'how' to perform the activity, and ways in which to pursue the activity in the face of obstacles, as well as expected challenges and possible solutions are discussed. ${ }^{31}$ The activity is supposed to be carried out by the participant prior to the next session. In session 5, a more challenging daily activity (related to concerns about falls) in daily life is first performed under the direct supervision of the facilitator. ${ }^{32}$ Furthermore, a DVD is used for modelling by presenting stories of other people reporting concerns about falls, and moreover, to encourage problem-solving skills of the participant, which is one of the core skills of selfmanagement. ${ }^{33}$ At last, the participant is encouraged to invite a significant other. This person (often a spouse or other relative, friend or neighbor) is present at the home visits and shown how to help and give support during the program. An overview of the program is shown in Table 1.

The AMB-Home program was facilitated by eight trained nurses, that were qualified in the field of geriatrics and work for home-care agencies. Facilitators received a two-day training in which the manual was studied. During the training, special attention was given to aspects such as motivational interviewing, behavioral change, and 'exposure in vivo' to feared activities ${ }^{32}$, by professionals in these particular fields. The facilitators were responsible for planning the sessions with the participant, according to the given format and time schedule. Throughout the start of the program, the researchers periodically observed the facilitators during their contacts with participants. Monthly group meetings of the facilitators and the researchers were held to evaluate and discuss the progress of the trial, the flow of participants, the program and the performance of the program by the facilitators.

\section{Outcomes}

\section{Effect evaluation}

Table 2 presents the outcomes of the effect evaluation. 
Table 1 Main topics of the in-home multicomponent cognitive behavioral intervention

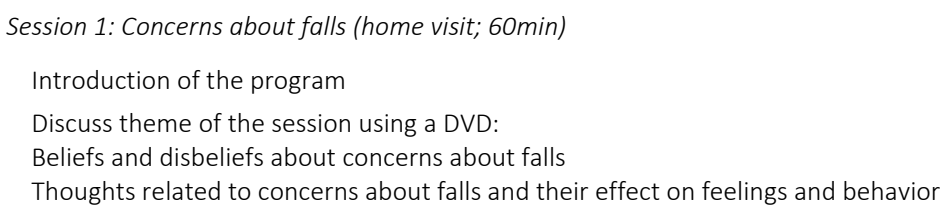

Session 2: Exploring thoughts and concerns about falls (home visit; 60min)

Review previous session and homework*

Discuss theme of the session using a checklist:

Thoughts and attitudes related to concerns about falls and challenging them

Adaptive responses to counter misconceptions about falls

Unhelpful thoughts and their effect on feelings and behavior

Identifying activities in which concerns about falls are experienced

Formulating an action plan to perform an activity safely in which concerns about falls are experienced*

Session 3: Physical exercise (telephone; 35min)

Review previous session and homework*

Discuss theme of the session using a checklist:

Misconceptions regarding physical exercise for older people

Potential consequences of inactivity and benefits of physical activity

Staying or becoming physically active to prevent falls

Recognizing and overcoming barriers to staying or becoming physically active

Formulating an action plan to perform a physical exercise activity in daily life*

Session 4: Asserting oneself (telephone; 35min)

Review previous session and homework*

Discuss theme of the session using a checklist and a leaflet about fall hazards:

Association between assertiveness and fall prevention

Potential barriers and benefits of being assertive

Reducing concerns about falls and falls-risks by being assertive

Recognizing potential environmental fall hazards in one's home and community

Formulating an action plan to be assertive related to fall prevention*

Session 5: Overcoming personal barriers (home visit; 75min)

Review previous session and homework*

Recognizing and overcoming personal barriers for performing activities of daily living

Performing an activity in which concerns about falls are experienced together with the facilitator\#

Shifting from self-defeating to self-motivating thoughts regarding activities in which concerns about falls are experienced

Formulating an action plan to repeat the activity performed in this session

Session 6: Safe behavior (telephone; 35min)

Review previous session and homework*

Discuss theme of the session using a checklist:

Recognizing risk-taking behavior in daily life

Identifying personal risk-taking behavior in daily life

Finding safe alternatives for unsafe behavior

Prioritizing fall-risk behavior and planning behavior-change strategies

Formulating an action plan to perform safe behavior* 
Session 7: Managing concerns about falls (telephone; 35min)

Review previous session and homework*

Shifting from self-defeating to self-motivating thoughts regarding activities in which concerns about falls are experienced

Finding personal solutions to perform activities safely in which concerns about falls are experienced

Review and evaluation of the program

Formulating an action plan to perform an activity safely in which concerns about falls are experienced*

* More explicitly addressed in the individual program compared to the group approach owing to the individual character of the program and the contracting.

\#New element in the individual program.

Note: several intervention elements of the group program were not included in the in-home program, among others: practicing simple physical exercises; explicit addressing of physical risk factors for falls; prevention strategies to minimize potential negative consequences of a fall; sharing experiences related to concerns about falls and fall prevention; a booster session after six months.

\section{Primary outcome measures}

The primary outcomes of the effect evaluation are concerns about falls and the avoidance of activity as a result of these concerns. Concerns about falls are assessed by the 16-item Falls Efficacy Scale-International (FES-I). Participants are asked to indicate how concerned they are about falling while carrying out several activities of daily living ( $1=$ not all concerned to 4 = very concerned). ${ }^{34,35}$ In addition, when people indicate that they are at least somewhat concerned about falling while carrying out an activity, people are asked to indicate to what extent they avoid the activity as a result of their concerns (FES-IAB; AB indicates Avoidance Behavior; 1 = never to 4 = often).

\section{Secondary outcome measures}

Secondary outcomes are disability and the number of falls. Disability is measured by the Groningen Activity Restriction Scale (GARS). ${ }^{36}$ The 18 items of the GARS measure disability in the area of ADL (Activities of Daily Living including mobility) as well as IADL (Instrumental Activities of Daily Living). Participants are asked if they are currently able to perform the activity ( $1=$ yes, fully independently to $4=$ no, only with help from others). The number of falls is registered continuously during the course of the trial by a fall calendar. A fall is defined as an event that results in a person coming to rest inadvertently on the ground or on another lower level. ${ }^{37}$ If a fall occurs, participants indicate on the calendar: (a) the location of the fall (indoor or outdoor); and (b) the number of times medical attention is received owing to the fall.

\section{Tertiary outcome measures}

The tertiary outcomes include: the perceived consequences of falling (CoF) with two 6item subscales ('loss of functional independence' and 'damage to identity') ${ }^{9}$; catastrophic beliefs about the consequences of a fall (CAFS: Catastrophizing About Falling Scale, 5 items) ${ }^{38}$; perceived control over falling (PCOF; 4 items) ${ }^{39}$; mastery $\left(7\right.$ items) ${ }^{40}$; 
feelings of anxiety and symptoms of depression with two 7-item subscales of the Hospital Anxiety and Depression Scale (HADS) ${ }^{41,42}$; social support interactions (SSL12-I: Social Support List of Interaction, 12 items) ${ }^{43}$; and health-related quality of life (SF-12: Health Survey, 12 items) $)^{44}$.

Table 2 Outcome measures of the effect evaluation

\begin{tabular}{|c|c|c|c|c|c|c|c|}
\hline Primary outcome measures & Instrument & No. of items & Range* & $\mathrm{S}$ & $\mathrm{B}$ & FU1 & FU2 \\
\hline concerns about falls & FES-I ${ }^{34,35,54}$ & 16 & $\underline{16}$ to 64 & - & $\mathrm{TI}$ & $\mathrm{TI}$ & $\mathrm{TI}$ \\
\hline $\begin{array}{l}\text { avoidance of activity owing to } \\
\text { concerns about falls }\end{array}$ & FES-IAB & 16 & $\underline{16}$ to 64 & - & $\mathrm{Tl}$ & $\mathrm{TI}$ & $\mathrm{TI}$ \\
\hline Secondary outcome measures & Instrument & No. of items & Range* & $\mathrm{S}$ & B & FU1 & FU2 \\
\hline activities of daily life & & 18 & $\underline{18}$ to 72 & - & $\mathrm{Tl}$ & $\mathrm{TI}$ & $\mathrm{TI}$ \\
\hline no. of falls & $\mathrm{GARS}^{36}$ & 1 & $\mathrm{~N} / \mathrm{A}$ & - & $\mathrm{C}>$ & $\mathrm{C}>$ & $C>$ \\
\hline - indoor & N/A & 1 & N/A & - & $C>$ & $C>$ & $C>$ \\
\hline - outdoor & $\mathrm{N} / \mathrm{A}$ & 1 & $\mathrm{~N} / \mathrm{A}$ & - & $C>$ & $C>$ & $C>$ \\
\hline $\begin{array}{l}\text { no. of times medical attention } \\
\text { required as a result of falls }\end{array}$ & $\mathrm{N} / \mathrm{A}$ & 1 & $\mathrm{~N} / \mathrm{A}$ & - & $C>$ & $C>$ & $C>$ \\
\hline Tertiary outcome measures & Instrument & No. of items & Range* & $\mathrm{S}$ & B & FU1 & FU2 \\
\hline $\begin{array}{l}\text { perceived consequences of falling } \\
\text { loss of functional independence } \\
\text { subscale }\end{array}$ & $\mathrm{CoF}^{9}$ & 6 & $\underline{6}$ to 24 & - & $\mathrm{Tl}$ & $\mathrm{TI}$ & $\mathrm{TI}$ \\
\hline $\begin{array}{l}\text { perceived consequences of falling } \\
\text { damage to identity subscale }\end{array}$ & $\mathrm{CoF}^{9}$ & 6 & $\underline{6}$ to 24 & - & $\mathrm{TI}$ & $\mathrm{TI}$ & TI \\
\hline $\begin{array}{l}\text { catastrophic beliefs about } \\
\text { consequences of a fall }\end{array}$ & $\mathrm{CAFS}^{38}$ & 5 & $\underline{5}$ to 20 & - & $\mathrm{TI}$ & $\mathrm{TI}$ & $\mathrm{TI}$ \\
\hline perceived control over falling & $\mathrm{PCOF}^{39}$ & 4 & $\underline{4}$ to 20 & - & $\mathrm{TI}$ & $\mathrm{TI}$ & $\mathrm{TI}$ \\
\hline mastery & $\begin{array}{l}\text { Personal Mastery } \\
\text { Scale }^{40}\end{array}$ & 7 & 7 to $\underline{35}$ & - & $\mathrm{TI}$ & $\mathrm{TI}$ & $\mathrm{TI}$ \\
\hline feelings of anxiety & HADS-A ${ }^{41,42}$ & 7 & $\underline{0}$ to 21 & - & $\mathrm{Tl}$ & $\mathrm{TI}$ & $\mathrm{TI}$ \\
\hline symptoms of depression & HADS-D ${ }^{41,42}$ & 7 & $\underline{0}$ to 21 & - & $\mathrm{TI}$ & $\mathrm{TI}$ & $\mathrm{TI}$ \\
\hline social support interactions & SSL $12-1^{43}$ & 12 & 12 to $\underline{48}$ & - & $\mathrm{TI}$ & $\mathrm{TI}$ & $\mathrm{TI}$ \\
\hline health-related quality of life & SF $-12^{44}$ & 12 & 0 to $\underline{100}$ & - & $\mathrm{Tl}$ & $\mathrm{TI}$ & $\mathrm{TI}$ \\
\hline Additional variables & Instrument & No. of items & Range* & $\mathrm{S}$ & B & FU1 & FU2 \\
\hline $\begin{array}{l}\text { demographic data } \\
\text { (age, gender, living situation, } \\
\text { educational level) }\end{array}$ & $\mathrm{N} / \mathrm{A}$ & 5 & N/A & SQ & - & - & - \\
\hline perceived general health & $\begin{array}{l}\text { Perceived general } \\
\text { health }^{24}\end{array}$ & 1 & $\underline{1}$ to 5 & SQ & - & - & - \\
\hline
\end{tabular}




\begin{tabular}{|c|c|c|c|c|c|c|c|}
\hline Additional variables & Instrument & No. of items & Range* & $S$ & B & FU1 & FU2 \\
\hline \multirow[t]{2}{*}{ cognitive impairment } & $\mathrm{AMT4}^{25}$ & 4 & N/A & - & $\mathrm{TI}$ & - & - \\
\hline & $\operatorname{TICS}^{26}$ & 11 & 0 to $\underline{41}$ & - & $\mathrm{TI}^{\#}$ & - & - \\
\hline chronic medical conditions & $\begin{array}{l}\text { Chronic medical } \\
\text { conditions } \\
\text { questionnaire }^{47}\end{array}$ & 5 & $\underline{0}$ to 5 & - & $\mathrm{TI}$ & - & - \\
\hline concerns about falls & $\mathrm{N} / \mathrm{A}$ & 1 & 1 to 6 & SQ & $\mathrm{TI}$ & $\mathrm{TI}$ & $\mathrm{TI}$ \\
\hline $\begin{array}{l}\text { avoidance of activity owing to } \\
\text { concerns about falls }\end{array}$ & $\mathrm{N} / \mathrm{A}$ & 1 & 1 to 6 & SQ & $\mathrm{TI}$ & $\mathrm{TI}$ & $\mathrm{TI}$ \\
\hline no. of falls in the previous 6 months & $\mathrm{N} / \mathrm{A}$ & 1 & 1 to 6 & $\mathrm{SQ}$ & $\mathrm{TI}$ & - & - \\
\hline no. of falls in the previous 5 months & $\mathrm{N} / \mathrm{A}$ & 1 & 1 to 6 & - & - & $\mathrm{TI}$ & - \\
\hline no. of falls in the previous 7 months & $\mathrm{N} / \mathrm{A}$ & 1 & 1 to 6 & - & - & - & $\mathrm{TI}$ \\
\hline expectations of intervention & $\begin{array}{l}\text { Adapted } \\
\text { expectations } \\
\text { questionnaire }^{48}\end{array}$ & 4 & 4 to $\underline{20}$ & - & $\mathrm{TI}$ & - & - \\
\hline
\end{tabular}

*The underlined scores indicate the most favorable scores; $\mathrm{N} / \mathrm{A}=$ not applicable; $\mathrm{S}=$ screening; $\mathrm{B}=$ baseline; FU1 = 5-month follow-up; FU2 = 12-month follow-up; $\mathrm{SQ}=$ screening questionnaire; $\mathrm{TI}$ = telephone interview; C> calendar (continuous registration); ${ }^{\#}$ Only assessed if participant fails on the AMT4

\section{Additional variables}

Several variables are assessed to provide insight into the population under study, and to interpret the outcomes of the study. The socio-demographic and health-related variables, assessed during the process of screening for eligibility, are: age, gender, living alone or not, educational level, perceived general health (item one of the MOS SF$20)^{24,45}$, and self-reported impaired vision and hearing ${ }^{46}$. Other health-related variables assessed during the baseline measurement are: chronic medical conditions (a 5-item checklist) ${ }^{47}$ and cognitive status (AMT4: shortened version of the Abbreviated Mental Test; 4 items and TICS: Telephone Interview Cognitive Status; 11 items) ${ }^{25,26}$. Furthermore, 1-item questions on concerns about falls, the avoidance of activities owing to these concerns and the number of falls are assessed at baseline, as well as, all follow-up measurements. Lastly, at the baseline, participants are asked about their outcome expectations with regard to the program. ${ }^{48}$

\section{Process evaluation}

To determine the feasibility of the program and to identify factors that may influence its effectiveness, the following outcomes of the process evaluation are assessed: characteristics of the population reached (reach); protocol adherence by facilitators (fidelity); protocol adherence by participants (dose received: exposure); participants and facilitators' opinion about the program and perceived benefits and achievements (dose received: satisfaction); and experienced barriers and potential solutions for these (barriers). ${ }^{21,49,50}$ Table 3 provides a detailed overview of the outcomes of the process evalua- 
tion and their operationalization during the course of the trial. Data is collected from participants in the program group, and from the facilitators.

Table 3 Outcome measures of the process evaluation

\begin{tabular}{ll}
\hline Component and definition & Operationalization \\
\hline Reach & \\
$\begin{array}{l}\text { Proportion of the intended } \\
\text { target population that } \\
\text { participated in the program }\end{array}$ & $\begin{array}{l}\text { Characteristics of participants and } \\
\text { facilitators }\end{array}$ \\
& $\begin{array}{l}\text { Number of participants that refused, } \\
\text { dropped out or completed the } \\
\text { program } \\
\end{array}$ \\
& Reasons for withdrawal
\end{tabular}

Measurement

SQ QCf $\vee$ Qf Qp If D

Fidelity

Extent to which the program Preparation time and duration of the was implemented as planned session

Per session component: extent to which carried out, duration and active participation by the participant

Extent to which the facilitator achieved to:

conveying information to the participant

having the participant phrase their important activities and how these activities could be performed safely and independently having the participant set goals regarding an action plan using motivational interviewing techniques

Dose received (exposure)

Extent of participants' active engagement in and

Overall opinion of the facilitator/participant regarding the receptiveness to the program participant's engagement in:

the program

the formulation of an action plan and carrying out an action plan

Use of materials

Exposure and adherence to homework

Extent to which the participant

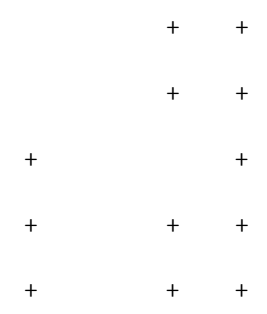




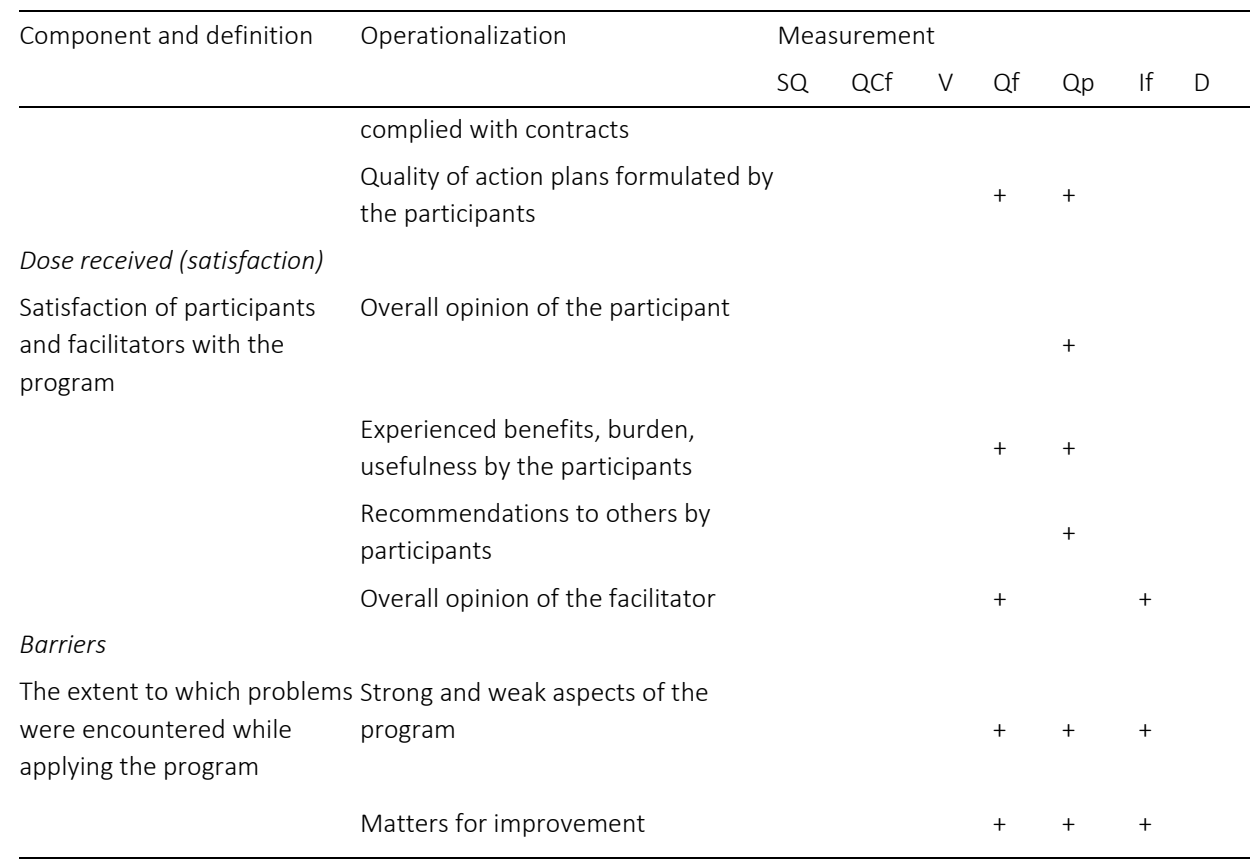

$\mathrm{SQ}=$ screening questionnaire filled in by participant before start program, QCf = questionnaire filled in by facilitator for each participant for each contact, $V=$ voice recording for selection of contacts, Qf = questionnaire filled in by facilitator for each participant after completion of program, $Q p=$ questionnaire filled in by participant after program, If = group interview with facilitators, and $D=$ data recorded by researchers during program period

\section{Economic evaluation}

A cost-effectiveness analysis is carried out in which costs are considered from a societal perspective. The economic evaluation measures and evaluates the 'real' costs. In this study, direct health-care costs are included; i.e. costs incurred by the in-home program and health-care costs incurred by the participants. The costs of the program consist of used materials, salaries of the facilitators, costs of training sessions for the facilitators etc. Health-care costs include hospital visits (inpatient and outpatient treatment), GP consultations, visits to paramedics, (nursing) home-care, informal care, and aids and appliances. In order to estimate the costs, the quantity of each resource will be multiplied by its assigned unit cost of price. Cost prices are obtained from the Dutch guidelines for cost analysis in health-care research. ${ }^{51,52}$ If such guidelines do not provide for specific health-care use, real costs or tariffs will be used to estimate costs.

\section{Data collection}

Data for the effect evaluation is gathered by means of telephone interviews which are conducted by trained interviewers, who are blinded for group allocation. For the assessment of fall accidents, participants received a fall calendar after the baseline meas- 
urement. Every month, a sheet of the calendar has to be returned via a freepost envelope. People are reminded by telephone after one-and-half weeks if a sheet is not returned.

The process evaluation data is gathered by several means. Participants who complete at least five sessions, fill in a questionnaire to report on the program's feasibility and usefulness. Facilitators receive a registration form for each participant to report on the time spent per session, the participant's adherence with regard to homework assignments and the extent to which the program is performed according to protocol. Voice recordings are used in a random selection of the sessions to gather objective data about the performance according to the protocol. Additionally, facilitators fill in questionnaires that assess their opinion about the program for each participant and their overall opinion of the program. Researchers conduct short telephone interviews to identify the reason(s) for withdrawal among people who do not complete the program. Lastly, the researchers conduct a final evaluation meeting with the facilitators to discuss the overall program.

The fall calendar mentioned before is also used for the collection of the data for the economic evaluation. Participants have to report their use of health-care services each month, in addition to their fall accidents.

Non-compliant participants of the program group are approached for all follow-up measurements, and participants with missing data are contacted to ensure completion of data, as recommended by Hollis and Campbell. ${ }^{53}$

Newsletters are sent 4 and 11 months after the baseline measurement, to keep the participants informed about the trial.

\section{Sample size and power}

Sample size calculations are based on outcomes of a previous study using the Falls Efficacy Scale-International (FES-I) among older people in the Netherlands. ${ }^{54}$ Two times 112 participants will provide $80 \%$ power at alpha 0.05 (one-tailed) to detect differences between the intervention and control groups' mean score of at least 3.8 points (SD is 11.4 equivalent with an effect size of 0.33 on the FES-I). However, a dropout rate of $20 \%$ during the study is expected, based on the experiences in the home visit study from Van Haastregt et al. ${ }^{55}$ Therefore, $2 \times 140$ participants are needed to enroll in the trial.

In a previous Dutch study, 54\% of the population reported fear of falling, 38\% reported related avoidance behavior, and $48 \%$ reported poor or fair perceived general health. ${ }^{8}$ Based on the experiences in that study and on the evaluation of AMB-NL ${ }^{15}$, we estimate that approximately $6 \%$ of the older people who return the screening questionnaire will meet all inclusion and exclusion criteria and will be interested in participating in the trial. With an estimated response rate of 55\%, a minimum sample of 8,200 older persons aged 70 or over needs to be approached with a screening questionnaire. 


\section{Analysis}

Descriptive techniques will be used to describe the study groups. Baseline variables will be compared, to detect differences between the participants of both groups at the start of the study. Data of the effect evaluation will be analyzed according to both the intention-to-treat and per-protocol principles. In the first analyses all participants will be included according to their original assignment. ${ }^{53}$ Participants of the intervention group who attended at least five of the seven program sessions will be included in the perprotocol analysis. Based on prior work, five sessions of the program are considered as sufficient program exposure. ${ }^{15,18}$ Mixed-effects regression analyses will be applied, to test for between-group differences with respect to the primary, secondary and tertiary outcome measures at all follow-up assessments. Models will be adjusted for the following covariates, considered as relevant for the outcomes ${ }^{8}$ : concerns about falls, age, gender, perceived general health, and number of falls in the past 6 months at baseline. Additional covariates will be included in the analysis if baseline differences are detected for variables relevant for the outcomes. The level of statistical significance will be set at 0.05 (one-tailed). Data on the process and economic evaluation will be analyzed and presented using descriptive techniques and appropriate statistical testing.

\section{Discussion}

A multicomponent cognitive behavioral in-home program has been developed to teach frail older people living in the community how to manage their concerns about falls and related activity avoidance. A trial was conducted to evaluate this program on effectiveness, feasibility and costs. The screening procedure for eligible participants started in March 2009. For practical reasons, the procedure is distributed across four cycles, that last of which was in December 2009. In the first cycle, 2,250 older people living in the south-east of the Netherlands received the screening questionnaire. Enrolment into the study was disappointing during this cycle. Therefore, we decided to send out more screening questionnaires than planned in the next cycles, and to broaden the inclusion criteria from regular to both some concerns about falls and some avoidance of activity. In addition, responders entered a draw to win one of the fifteen gift vouchers worth 25 Euro. Eventually, 11,490 older people received the screening questionnaire across the four cycles. The response rate was $52.6 \%$ and by applying the modified inclusion criteria, 389 participants were included into the study.

Changes made in the treatment protocol of the group program while adapting it for the individual in-home application may influence effectiveness. First, the physical exercises from the group approach were removed from the program, because the facilitator could not monitor them adequately during the telephone contacts. Instead, more attention is given to action plans and overcoming a more challenging (fear-related) problem in daily life in session 5. Second, by sending huge numbers of questionnaires, we 
choose a screening procedure to include enough participants in a relatively short period of time. This is also the reason why participants did not receive a personal assessment in which they are tested on, for example, physical performances, such as balance and strength, and motivation. With this procedure, we may have missed helpful information about how realistic the participants' concerns about falls are and, how much they are prepared to do something about their concerns. In the future, possibilities for testing participants' physical performance ${ }^{56}$ and motivation at the start of the program might be explored ${ }^{57}$. Third, to make implementation in the Dutch health-care setting more acceptable, we have chosen for community nurses as facilitators. Yet, these nurses have little experience of facilitating behavioral change; instead, they are usually trained to provide care and information. In this program, they were also expected to act as a personal coach to the participants, and to encourage self-management skills. This might have put additional demands on them as facilitators, for which they may not have been trained in the past. Therefore, and to increase the treatment integrity of the program, nurses received specific training prior to the start of the project. Lastly, the use of telephone contacts as part of the program is rather new among this older population, in particular, and nurses from home-care organizations in the Netherlands. If successful, the results might lead to a more cost-effective program and promising prospects for further use of telephone contacts in programs with frail older people in the future.

\section{Progress of the study}

The baseline measurements started in March 2009. Data on the effect, process and economic evaluation are expected to be available in 2012.

\section{Future implementation}

During the last two years the successful group program AMB-NL has been implemented nationwide into the Dutch health-care setting. Details about the program are presented on the Dutch website www.zichtopevenwicht.nl. If the results of the current trial show effectiveness and feasibility of AMB-Home, then the in-home program can be offered to people who are not able or willing to participate in the group program.

\section{Acknowledgements}

We would like to thank the municipal registry offices of Maastricht, Heerlen and SittardGeleen, the Centre for Data and Information Management (MEMIC) and research assistant Yvonne van Eijs for their assistance in this study. We would also like to thank the experts Jolanda van Haastregt (Vivre) and José Grouls (Meandergroep) who, in addition to the authors of this article, contributed to the development of the program protocol. This research has been funded by ZonMw, the Netherlands Organization for Health Research and Development (grant 120610001). The participation of author JV was sup- 
ported by the Odysseus Grant 'The Psychology of Pain and Disability Research Program' funded by the Research Foundation, Flanders, Belgium (FWO Vlaanderen). 


\section{References}

1. Masud T, Morris RO: Epidemiology of falls. Age Ageing. 2001, 30 Suppl 4:3-7.

2. Rubenstein LZ: Falls in older people: epidemiology, risk factors and strategies for prevention. Age Ageing. 2006, 35 Suppl 2:ii37-ii41.

3. Arfken CL, Lach HW, Birge SJ, Miller JP: The prevalence and correlates of fear of falling in elderly persons living in the community. Am J Public Health. 1994, 84(4):565-570.

4. Fletcher PC, Hirdes JP: Restriction in activity associated with fear of falling among community-based seniors using home care services. Age Ageing. 2004, 33(3):273-279.

5. Howland J, Lachman ME, Peterson EW, Cote J, Kasten L, Jette A: Covariates of fear of falling and associated activity curtailment. Gerontologist. 1998, 38(5):549-555.

6. Murphy SL, Williams CS, Gill TM: Characteristics associated with fear of falling and activity restriction in community-living older persons. J Am Geriatr Soc. 2002, 50(3):516-520.

7. Tinetti ME, Mendes de Leon CF, Doucette JT, Baker DI: Fear of falling and fall-related efficacy in relationship to functioning among community-living elders. J Gerontol. 1994, 49(3):M140-147.

8. Zijlstra GA, van Haastregt JC, van Eijk JT, van Rossum E, Stalenhoef PA, Kempen GI: Prevalence and correlates of fear of falling, and associated avoidance of activity in the general population of communityliving older people. Age Ageing. 2007, 36(3):304-309.

9. Yardley L, Smith $\mathrm{H}$ : A prospective study of the relationship between feared consequences of falling and avoidance of activity in community-living older people. Gerontologist. 2002, 42(1):17-23.

10. Cumming RG, Salkeld G, Thomas M, Szonyi G: Prospective study of the impact of fear of falling on activities of daily living, SF-36 scores, and nursing home admission. J Gerontol A Biol Sci Med Sci. 2000, 55(5):M299-305.

11. Delbaere K, Close JC, Brodaty H, Sachdev P, Lord SR: Determinants of disparities between perceived and physiological risk of falling among elderly people: cohort study. BMJ. 2010, 341:c4165.

12. Friedman SM, Munoz B, West SK, Rubin GS, Fried LP: Falls and fear of falling: which comes first? A longitudinal prediction model suggests strategies for primary and secondary prevention. J Am Geriatr Soc. 2002, 50(8):1329-1335.

13. Delbaere K, Sturnieks DL, Crombez G, Lord SR: Concern about falls elicits changes in gait parameters in conditions of postural threat in older people. J Gerontol a-Biol. 2009, 64(2):237-242.

14. Hadjistavropoulos T, Delbaere K, Fitzgerald TD: Reconceptualizing the role of fear of falling and balance confidence in fall risk. J Aging Health. 2011, 23(1):3-23.

15. Zijlstra GA, van Haastregt JC, Ambergen T, van Rossum E, van Eijk JT, Tennstedt SL, Kempen GI: Effects of a multicomponent cognitive behavioral group intervention on fear of falling and activity avoidance in community-dwelling older adults: results of a randomized controlled trial. J Am Geriatr Soc. 2009, 57(11):2020-2028.

16. Brouwer BJ, Walker C, Rydahl SJ, Culham EG: Reducing fear of falling in seniors through education and activity programs: A randomized trial. J Am Geriatr Soc. 2003, 51(6):829-834.

17. Clemson L, Cumming RG, Kendig H, Swann M, Heard R, Taylor K: The effectiveness of a communitybased program for reducing the incidence of falls in the elderly: a randomized trial. J Am Geriatr Soc. 2004, 52(9):1487-1494.

18. Tennstedt S, Howland J, Lachman M, Peterson E, Kasten L, Jette A: A randomized, controlled trial of a group intervention to reduce fear of falling and associated activity restriction in older adults. J Gerontol B Psychol Sci Soc Sci. 1998, 53(6):P384-392.

19. Bula CJ, Monod S, Hoskovec C, Rochat S: Interventions aiming at balance confidence improvement in older adults: an updated review. Gerontology. 2011, 57(3):276-286.

20. Zijlstra GA, Tennstedt SL, van Haastregt JC, van Eijk JT, Kempen GI: Reducing fear of falling and avoidance of activity in elderly persons: the development of a Dutch version of an American intervention. Patient Educ Couns. 2006, 62(2):220-227. 
21. van Haastregt JC, Zijlstra GA, van Rossum E, van Eijk JT, de Witte LP, Kempen GI: Feasibility of a cognitive behavioural group intervention to reduce fear of falling and associated avoidance of activity in community-living older people: a process evaluation. BMC Health Serv Res. 2007, 7:156.

22. Sattin RW, Easley KA, Wolf SL, Chen Y, Kutner MH: Reduction in fear of falling through intense tai chi exercise training in older, transitionally frail adults. J Am Geriatr Soc. 2005, 53(7):1168-1178.

23. Dorresteijn TA, Rixt Zijlstra GA, Van Eijs YJ, Vlaeyen JW, Kempen GI: Older people's preferences regarding programme formats for managing concerns about falls. Age Ageing. 2012, 41(4):474-481.

24. Stewart AL, Hays RD, Ware JE, Jr.: The MOS short-form general health survey. Reliability and validity in a patient population. Med Care. 1988, 26(7):724-735.

25. Swain DG, Nightingale PG: Evaluation of a shortened version of the abbreviated mental test in a series of elderly patients. Clin Rehabil. 1997, 11(3):243-248.

26. Kempen GI, Meier AJ, Bouwens SF, van Deursen J, Verhey FR: [The psychometric properties of the Dutch version of the telephone interview cognitive status (TICS)]. Tijdschr Gerontol Geriatr. 2007, 38(1):38-45.

27. Zijlstra GA, van Haastregt JC, van Eijk JT, de Witte LP, Ambergen T, Kempen GI: Mediating effects of psychosocial factors on concerns about falling and daily activity in a multicomponent cognitive behavioral group intervention. Aging Ment Health. 2011, 15(1):68-77.

28. Lachman ME, Jette A, Tennstedt S, Howland J, Harris BA, Peterson E: A cognitive-behavioural model for promoting regular physical activity in older adults. Psychol Health Med. 1997, 2(3):251-261.

29. Resnicow K, Dilorio C, Soet JE, Borrelli B, Hecht J, Ernst D: Motivational interviewing in health promotion: it sounds like something is changing. Health Psychol. 2002, 21(5):444-451.

30. Delbaere K, S TS, Lord SR: Development and initial validation of the iconographical falls efficacy scale. J Gerontol a-Biol. 2011, 66(6):674-680.

31. Ziegelmann JP, Lippke S, Schwarzer R: Adoption and maintenance of physical activity: planning interventions in young, middle-aged, and older adults. Psychology and Health. 2006, 21(2):145-163.

32. Vlaeyen JWS, de Jong J, Geilen M, Heuts PHTG, van Breukelen G: The treatment of fear of movement/(re)injury in chronic low back pain: further evidence on the effectiveness of exposure in vivo. Clin J Pain. 2002, 18(4):251-261.

33. Lorig KR, Holman H: Self-management education: history, definition, outcomes, and mechanisms. Ann Behav Med. 2003, 26(1):1-7.

34. Yardley L, Beyer N, Hauer K, Kempen G, Piot-Ziegler C, Todd C: Development and initial validation of the falls efficacy scale-international (FES-I). Age Ageing. 2005, 34(6):614-619.

35. Kempen GI, Zijlstra GA, van Haastregt JC: [The assessment of fear of falling with the falls efficacy scaleinternational (FES-I). Development and psychometric properties in Dutch elderly]. Tijdschr Gerontol Geriatr. 2007, 38(4):204-212.

36. Kempen GI, Miedema I, Ormel J, Molenaar W: The assessment of disability with the Groningen activity restriction scale. Conceptual framework and psychometric properties. Soc Sci Med. 1996, 43(11):16011610.

37. The prevention of falls in later life: A report of the kellogg international work group on the prevention of falls by the elderly. Dan Med Bull. 1987, 34 Suppl 4:1-24.

38. Delbaere K, Crombez G, van Haastregt JCM, Vlaeyen JWS: Falls and catastrophic thoughts about falls predict mobility restriction in community-dwelling older people: a structural equation modelling approach. Aging Ment Health. 2009, 13(4):587-592.

39. Lawrence RH, Tennstedt SL, Kasten LE, Shih J, Howland J, Jette AM: Intensity and correlates of fear of falling and hurting oneself in the next year: baseline findings from a Roybal Center fear of falling intervention. J Aging Health. 1998, 10(3):267-286.

40. Pearlin LI, Schooler C: The structure of coping. J Health Soc Behav. 1978, 19(1):2-21.

41. Bjelland I, Dahl AA, Haug TT, Neckelmann D: The validity of the hospital anxiety and depression scale. An updated literature review. J Psychosom Res. 2002, 52(2):69-77.

42. Spinhoven P, Ormel J, Sloekers PPA, Kempen GIJM, Speckens AEM, VanHemert AM: A validation study of the hospital anxiety and depression scale (HADS) in different groups of Dutch subjects. Psychol Med. 1997, 27(2):363-370. 
43. Kempen GIJM, Vaneijk LM: The psychometric properties of the SSL12-I, a short scale for measuring social support in the elderly. Social Indicators Research. 1995, 35(3):303-312.

44. Gandek B, Ware JE, Aaronson NK, Apolone G, Bjorner JB, Brazier JE, Bullinger M, Kaasa S, Leplege A, Prieto $L$ et al: Cross-validation of item selection and scoring for the SF-12 health survey in nine countries: results from the IQOLA Project. International quality of life assessment. J Clin Epidemiol. 1998, 51(11):1171-1178.

45. Kempen GI: [Assessment of health status of the elderly. Application of a Dutch version of the MOS scale]. Tijdschr Gerontol Geriatr. 1992, 23(4):132-140.

46. van Sonsbeek JLA: Methodological aspects and content of the OECD-longterm disability indicator. CBS Maandberichten Gezondheid. 1988, 6:4-17.

47. CBS: Gezondheidsenquete 1989 (Health Interview Survey). Voorburg/Heerlen, The Netherlands; 1989.

48. Goossens MEJB, Vlaeyen JWS, Hidding A, Kole-Snijders A, Evers SMAA: Treatment expectancy affects the outcome of cognitive-behavioral interventions in chronic pain. Clin J Pain. 2005, 21(1):18-26.

49. Zijlstra GAR, van Rens GHMB, Scherder EJA, Brouwer DM, van der Velde J, Verstraten PFJ, Kempen GIJM: Effects and feasibility of a standardised orientation and mobility training in using an identification cane for older adults with low vision: design of a randomised controlled trial. BMC Health Serv Res. 2009, 9:153.

50. Saunders RP, Evans MH, Joshi P: Developing a process-evaluation plan for assessing health promotion program implementation: a how-to guide. Health Promot Pract. 2005, 6(2):134-147.

51. Oostenbrink JB, Koopmanschap MA, Rutten FFH: Handleiding voor kostenonderzoek: methoden en richtlijnprijzen voor economische evaluaties in de gezondheidszorg. Amstelveen: College voor zorgverzekeringen; 2000.

52. Oostenbrink JB, Koopmanschap MA, Rutten FF: Standardisation of costs: the Dutch manual for costing in economic evaluations. Pharmacoeconomics. 2002, 20(7):443-454.

53. Hollis S, Campbell F: What is meant by intention to treat analysis? Survey of published randomised controlled trials. BMJ. 1999, 319(7211):670-674.

54. Kempen GI, Todd CJ, Van Haastregt JC, Zijlstra GA, Beyer N, Freiberger E, Hauer KA, Piot-Ziegler C, Yardley L: Cross-cultural validation of the falls efficacy scale international (FES-I) in older people: results from Germany, the Netherlands and the UK were satisfactory. Disabil Rehabil. 2007, 29(2):155-162.

55. van Haastregt JC, Diederiks JP, van Rossum E, de Witte LP, Voorhoeve PM, Crebolder HF: Effects of a programme of multifactorial home visits on falls and mobility impairments in elderly people at risk: randomised controlled trial. BMJ. 2000, 321(7267):994-998.

56. Persad CC, Cook S, Giordani B: Assessing falls in the elderly: should we use simple screening tests or a comprehensive fall risk evaluation? Eur J Phys Rehabil Med. 2010, 46(2):249-259.

57. Prochaska JO, DiClemente CC: Stages and processes of self-change of smoking: toward an integrative model of change. J Consult Clin Psychol. 1983, 51(3):390-395. 


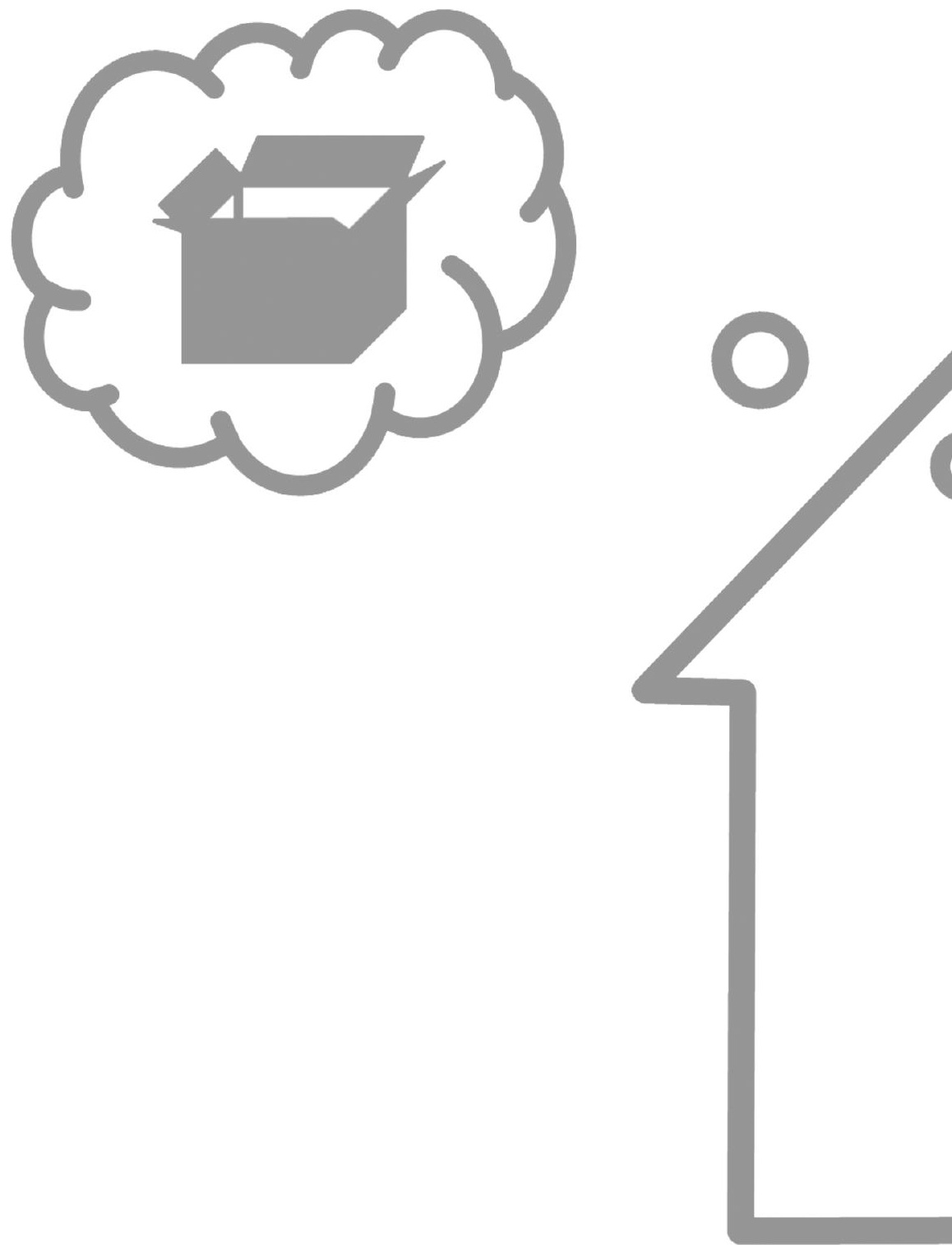


\section{Chapter 4}

\section{Feasibility of a nurse-led in-home cognitive behavioral program to manage concerns about falls in frail older people: a process evaluation}




\begin{abstract}
Concerns about falls and related avoidance of activities are common problems among older people living in the community. In this study we examined the feasibility and acceptability of AMB-Home (the Dutch in-home version of A Matter of Balance), a nurseled in-home cognitive behavioral program developed for frail community-living older people with concerns about falls and related activity avoidance. The multicomponent program consisted of seven individual sessions, including three home visits and four telephone contacts. Data were collected from eight nurses and 194 participants. Generally, the program was considered as acceptable and feasible by both the nurses and the participants. When AMB-Home turns out to be effective, the implementation of a finetuned version of this in-home program in regular health care, would be a natural next step.
\end{abstract}




\section{Background}

Concerns about falls, also frequently referred to as fear of falling, are present in $20-80 \%$ of community-living older people. ${ }^{1,2}$ Around $40 \%$ of older persons report restricting their activities because of these concerns. ${ }^{3-5}$ A fall can lead to concerns about falls, although such concerns also occur in people who have not fallen. ${ }^{5,6}$ Moreover, concerns about falls can result in future falls ${ }^{7}$, even without a physiological fall risk ${ }^{8}$. These concerns can further lead to decreased balance performance, decreased mobility and social participation, functional decline, low quality of life, and institutionalization. ${ }^{7-12}$

Diverse interventions have been developed and tested to decrease concerns about falls and showed varying effects. ${ }^{13,14}$ A program which consistently showed favorable effects on concerns about falls is the multicomponent, cognitive behavioral group program 'A Matter of Balance'. ${ }^{15-22}$ The Dutch version $\left(\mathrm{AMB}-\mathrm{NL}^{23}\right.$ ), for example, was effective in reducing concerns about falls, activity avoidance, and recurrent falls in Dutch community-dwelling older people. ${ }^{21}$ This nurse-led group program focuses on increasing self-efficacy and controlling the risk of falling, setting realistic goals to increase activity, adapting the environment to reduce the fall-risk, and promoting physical activity to increase strength and balance. AMB-NL is not only effective but is regarded as feasible and beneficial by participants and nurses. ${ }^{24}$ After a successful implementation strategy, the program is now widely available within the Dutch health care system (see 'Zicht op Evenwicht', the Dutch name for AMB-NL, on www.trimbos.nl). ${ }^{22}$

However, in a randomized controlled trial assessing the effects of this program, approximately $40 \%$ of the participants attended fewer than five of the eight program sessions, mainly because of health problems ${ }^{24}$, which suggests that despite its favorable outcomes, the program may benefit from a version tailored to frail populations. Such a version also may also appeal to people who dislike programs in a group format. In a recent study, community-living older people preferred a program format of home visits over a group program. ${ }^{25}$

Therefore, we developed an individual, home-based, multi-component, cognitive behavioral program (AMB-Home) based on AMB-NL, to facilitate reduced concerns about falls in mainly frail older people who live independently in the community and have a need or a preference for an individualized approach. ${ }^{26}$ The program is delivered by community nurses specializing in geriatric care who are employed by local homecare organizations.

In this article we present the results of a process evaluation of the AMB-Home program, which was implemented in three communities in the Netherlands. In our process evaluation we focused on several key process elements derived from prior research $^{27-30}$ : reach, fidelity, dose received - exposure, dose received - satisfaction, and barriers. These elements were measured: as participation of the target population in the program (reach); implementation of the program as planned (fidelity); participants' engagement in and receptivity to the program (dose received - exposure); participants' 
and facilitators' satisfaction with the program (dose received - satisfaction); and aspects of the program that need improvement prior to nationwide implementation (barriers). In general, a process evaluation gives an understanding of the feasibility and acceptability of a program and its strong and weak aspects and may lead to recommendations for improvement of the program, which could be helpful for successful largescale implementation. ${ }^{28,29}$

\section{Methods}

\section{Study design}

This process evaluation was a descriptive mixed methods study in which quantitative and qualitative data were gathered alongside a two-group randomized controlled trial. ${ }^{26}$ We focused on the participants who were allocated to the intervention group and the nurses, that is, the facilitators, who provided the intervention program. The design of the trial is described in more detail elsewhere. ${ }^{26}$ The Medical Ethics Committee of the Maastricht University Medical Centre in the Netherlands approved this study.

\section{Sample}

\section{Participants}

For this process evaluation, 194 of the 389 participants who were randomly assigned to the intervention group of the randomized controlled trial ${ }^{26}$ were included (see Figure 1). The recruitment of participants for this trial was performed in four consecutive cycles between March and December 2009 in three communities in the south of the Netherlands, that is, Maastricht, Sittard-Geleen, and Heerlen. The municipal registry offices of these communities selected at random a total of 11,490 addresses of communitydwelling people of 70 years or over. To screen for eligibility, these people received a short postal questionnaire with a free return envelope, as well as information about the trial and an informed consent form. After a fortnight, reminder letters were sent to those who failed to respond. The questionnaire included socio-demographic and fallrelated variables and inclusion and exclusion criteria for participation in the trial. In a telephone interview prior to randomization, we assessed cognitive impairment.

Older people were included if they met all of the following criteria: (1) reported at least some concerns about falls; (2) reported at least some associated avoidance of activity; (3) perceived their general health as fair or poor ${ }^{31}$; (4) lived in the community; (5) 70 years of age or older; and (6) were willing to participate (i.e., signed an informed consent form). People were excluded if they were confined to bed, wheelchair-bound, waiting for a nursing home admission, reported substantial hearing or vision impairments ${ }^{32}$ or failed the shortened version of the Abbreviated Mental Test (AMT4) ${ }^{33}$ to assess cognitive impairment and, subsequently, the Telephone Interview Cognitive Status $(\text { TICS })^{34}$. 


\section{Facilitators}

The facilitators of the intervention program were eight community nurses who specialized in geriatric care and were employed by three local homecare organizations in the three communities. Before conducting the intervention, facilitators received 2 days of training. During the training, the manual was studied, and professionals in the fields of motivational interviewing, behavioral change, and 'exposure in-vivo' provided workshops.

The facilitators were responsible for scheduling and conducting the sessions with the participants according to the program manual and timetable. Additionally, facilitators were expected to be empathetic and to motivate and challenge the participants during the program sessions.

In monthly group meetings, experiences of the facilitators with the program were exchanged and the progress of the trial was evaluated, including the flow of participants. During the first 4 months of the intervention period (the full intervention period of the trial lasted from May 2009 to May 2010) the first author also periodically observed the facilitators during their sessions with participants. These observations were intended to get an impression of how the program was implemented in practice and to give some feedback to the facilitators.

Seven of the eight facilitators (88\%) were female. The mean age of facilitators was 41 years (range 24-57). Their average years of experience in geriatric nursing was 12 (range 3-26) and their mean work week consisted of 29 hours (range 12-36).

\section{Intervention}

The intervention is an in-home, cognitive behavioral program (AMB-Home) that is designed to help participants learn how to deal with their concerns about falls and related avoidance of activity, in order to safely increase their physical, social, and functional activities. The program employs the following strategies: (1) restructuring misconceptions to promote a realistic view of fall risk and make concerns about falls controllable; (2) setting realistic goals for increasing activity and safe behavior; (3) adapting the environment to reduce fall risk; (4) promoting increased participation in daily life activities; and (5) gradually increasing exposure to feared activities. ${ }^{19,21,26}$

AMB-Home consists of seven individual sessions. The first two sessions are home visits of 60 minutes each, followed by two telephone contacts of 35 minutes, a third home visit of 75 minutes, and then two telephone contacts of each 35 minutes again. ${ }^{26}$ The first four sessions occur weekly, and the last three sessions are every 2 weeks.

During each session, one main theme is addressed. The themes of the program are: concerns about falls; thoughts about falling; physical exercise; asserting oneself; overcoming personal barriers; safe behavior; and managing concerns about falls. Each session has a similar structure with the following sequence of elements: reviewing the previous session; discussing the current session's theme; and formulating an action plan related to the theme that was discussed and that comprises a personal, tailor-made activity. 
Compared to the duration of the telephone contacts, the home visits are longer by 25 to 40 minutes. In the first two home visits (session 1 and 2) this extra time serves to establish a basis for trust between the participant and facilitator and to formulate realistic personal program goals regarding daily activities, for which the participant wants to overcome his or her concerns about falls. In the third home visit (session 5) the extra time enables the supervised performance of an activity in which the participant experiences concerns about falls, for example, walking down the stairs (gradual exposure).

A variety of techniques is used to encourage active participation and behavior change, including a DVD on role modeling, problem solving, planning, and motivational interviewing. The participants receive homework assignments between the sessions. These assignments include reading informative leaflets, filling in checklists to become aware of their beliefs about the themes of the sessions, and executing personal action plans. Participants are encouraged to invite significant others (e.g., a spouse, other relative, friend, or neighbor) to be present during the home visits. This significant other may provide help and support during and after the program. More details about the program are described elsewhere. ${ }^{26}$

\section{Data collection}

The theoretical key elements of process evaluation, as described by Baranowski and Stables $(2000)^{27}$ and Linnan and Steckler $(2002)^{28}$, were used for the development of the questionnaires. Table 1 provides a detailed overview of the operationalization of the elements (reach, fidelity, dose received-exposure, dose received-satisfaction, and barriers) into measurable outcomes.

Before randomization, the following background characteristics were collected from the participants by means of the short postal screening questionnaire (SQ): age, gender, living situation, educational level, perceived general health, number of falls in the past 6 months, concerns about falls and avoidance of activity due to concerns about falls.

The remaining data were collected only from the participants in the intervention group and from the facilitators. Participants who received at least five of the seven sessions received a questionnaire about the program's feasibility and usefulness ( $Q p$ see Table 1). Facilitators received a registration form for each participant and were asked to report on the time spent per session, the participant's adherence with regard to homework assignments, and the extent to which the program was performed according to protocol (QSf). Small voice recorders were used to gather objective data about the facilitator's performance according to the protocol. The facilitators audiotaped the sessions, if the participant had agreed to this (A). Additionally, facilitators completed questionnaires on their opinion of the usefulness of the program for each participant (Qf). Researchers conducted short telephone interviews to collect data about the reason(s) for withdrawal among participants who did not complete the program (D). Last, the researchers conducted a final group interview with the facilitators to 
discuss their overall experiences with the program (If). The data collection for the process evaluation occurred between March 2009 and May 2010.

\section{Data analysis}

Quantitative data (e.g., duration of the sessions, perceived benefits) were analyzed by means of descriptive statistics. We examined the differences between the group who completed the program and those who did not using the chi-square test and one-way ANOVA. A Wilcoxon matched-pairs signed ranks test was used to test for differences on the outcomes based on dose received as reported by participants and facilitators. Qualitative data (answers to open-ended questions in the self-administered questionnaires and the final group interview) were categorized. To compare the facilitators' selfreported protocol adherence to that revealed in the audio recordings of the sessions, two research assistants independently scored a random sample of the audio recordings using the same questionnaire as facilitators used in their self-ratings of the key program components. A total of 42 audio recordings was randomly selected based on the available recordings of six facilitators and included one example of each session (1-7) for every facilitator. The research assistants were asked to reach consensus on each component if their individual scores differed initially.

\section{Results}

\section{Reach}

Figure 1 displays the number of participants who enrolled in the trial, completed the program and received less than five sessions. In addition, the reasons for withdrawal are shown. Of the 194 participants randomly assigned to the intervention group, 117 participants $(60 \%)$ completed the program (i.e., received at least five of the seven sessions) and received a written questionnaire directly after the program. Four questionnaires were not returned. Main reasons for withdrawal from the program were lost interest $(n=27)$, health problems $(n=16)$, and perceived burden $(n=8)$.

The characteristics of participants in the intervention group are described in Table 2. Participants who received five or more sessions (column 2 program completed) did not significantly differ from those who received fewer than five sessions (column 3 program dropout). 
Table 1 Outcome measures of the process evaluation

\begin{tabular}{|c|c|c|c|c|c|c|c|}
\hline \multirow[t]{2}{*}{ Component and definition } & \multirow[t]{2}{*}{ Operationalization } & \multicolumn{6}{|c|}{ Measurement } \\
\hline & & SQ & QSf & A & Qf & Qp If & $\mathrm{D}$ \\
\hline \multicolumn{8}{|l|}{ Reach } \\
\hline \multirow{3}{*}{$\begin{array}{l}\text { Proportion of the intended } \\
\text { target population who } \\
\text { participated in the program }\end{array}$} & $\begin{array}{l}\text { Number of participants who refused, } \\
\text { dropped out or completed the program }\end{array}$ & & & & & & + \\
\hline & Reasons for withdrawal & & & & & & + \\
\hline & $\begin{array}{l}\text { Characteristics of participants and } \\
\text { facilitators }\end{array}$ & + & & & & & + \\
\hline \multicolumn{8}{|l|}{ Fidelity } \\
\hline \multicolumn{2}{|c|}{ Extent to which the program Duration of the session } & & + & & & & \\
\hline \multirow[t]{5}{*}{$\begin{array}{l}\text { was implemented as } \\
\text { planned }\end{array}$} & $\begin{array}{l}\text { Performance according to protocol of main } \\
\text { components per session }\end{array}$ & & + & + & & & \\
\hline & Extent to which the facilitator managed to: & & & & & & \\
\hline & $\begin{array}{l}\text { let the participant phrase his/her } \\
\text { important activities how to perform } \\
\text { activities safely and independently }\end{array}$ & & & + & + & & \\
\hline & $\begin{array}{l}\text { let the participant set goals regarding an } \\
\text { action plan }\end{array}$ & & & + & + & & \\
\hline & use motivational interviewing techniques & & & + & + & & \\
\hline
\end{tabular}

Dose received (exposure)

Extent of active engagement Overall opinion of the facilitator and in and receptiveness to the participant regarding the participant's program by participants engagement in the program

Adherence to homework, e.g. formulation and execution of an action plan

Goals reached

Use of materials

Dose received (satisfaction)

Satisfaction of participants and facilitators with the program

Overall opinion of the participant and facilitator

Experienced benefits, burden, usefulness by the participants

Recommendations to others by participants

\section{Barriers}

The extent to which Barriers in applying the program, e.g. problems were encountered reasons for deviations from protocol while applying the program Matters for improvement

Note: adapted version of outcome measures of the process evaluation as published earlier (Dorresteijn, et al., 2011).

$\mathrm{SQ}=$ screening questionnaire filled in by participant before start program, $\mathrm{QSf}=$ questionnaire filled in by facilitator per participant per session, $A=$ audio recording per session (selection of sessions), $Q f=$ questionnaire filled in by facilitator per participant after completion of program, $Q p=$ questionnaire filled in by participant after program, If = group interview with facilitators, and $D=$ data recorded by researchers during program period 
Table 2 Reach: characteristics of the participants in the intervention group $(n=194)$

\begin{tabular}{|c|c|c|c|c|c|}
\hline Participants & \multicolumn{2}{|c|}{$\begin{array}{l}\text { Program } \\
\text { completed* } \\
(n=117)\end{array}$} & \multicolumn{2}{|c|}{$\begin{array}{l}\text { Program dropout** } \\
(\mathrm{n}=77)\end{array}$} & $p$-value \\
\hline \multicolumn{6}{|l|}{ Demographic } \\
\hline \multirow[t]{2}{*}{ Mean age in years (SD) } & & & & & 0.78 \\
\hline & 78.5 & $(5.5)$ & 78.2 & $(5.2)$ & \\
\hline Gender (\%) & & & & & 0.26 \\
\hline Male & 41 & $(35.0)$ & 21 & $(27.3)$ & \\
\hline Female & 76 & $(65.0)$ & 56 & $(72.6)$ & \\
\hline Living situation (\%) & & & & & 0.17 \\
\hline Not alone & 46 & $(39.3)$ & 39 & $(50.6)$ & \\
\hline Alone & 71 & $(60.7)$ & 38 & $(49.4)$ & \\
\hline Educational level (\%) & & & & & 0.17 \\
\hline Low & 68 & $(58.1)$ & 43 & $(57.3)$ & \\
\hline Middle & 29 & $(24.8)$ & 26 & (34.7) & \\
\hline High & 20 & $(17.1)$ & 7 & $(9.2)$ & \\
\hline \multicolumn{6}{|l|}{$\underline{\text { Health-related }}$} \\
\hline Perceived general health (\%) & & & & & 0.96 \\
\hline Fair & 100 & $(85.5)$ & 66 & $(85.7)$ & \\
\hline Poor & 17 & $(14.5)$ & 11 & $(14.3)$ & \\
\hline \multicolumn{6}{|l|}{ Fall-related } \\
\hline Falls in the past 6 months (\%) & & & & & 0.98 \\
\hline Never & 39 & $(33.6)$ & 25 & (32.9) & \\
\hline Once & 33 & $(28.4)$ & 21 & $(27.6)$ & \\
\hline More than once & 44 & (37.9) & 30 & $(39.5)$ & \\
\hline Concerns about falls (\%) & & & & & 0.64 \\
\hline Sometimes & 53 & $(45.3)$ & 38 & $(49.4)$ & \\
\hline Regular & 30 & $(25.6)$ & 23 & $(29.9)$ & \\
\hline Often & 21 & $(17.9)$ & 10 & $(13.0)$ & \\
\hline Very often & 13 & $(11.1)$ & 6 & $(7.8)$ & \\
\hline Avoidance of activities (\%) & & & & & 0.54 \\
\hline Sometimes & 55 & $(47.0)$ & 30 & (39.0) & \\
\hline Regular & 33 & $(28.2)$ & 29 & $(37.7)$ & \\
\hline Often & 16 & $(13.7)$ & 11 & $(14.3)$ & \\
\hline Very often & 13 & $(11.1)$ & 7 & (9.1) & \\
\hline
\end{tabular}

Note: all numbers and percentages may not add up to final numbers due to missing data.

* Participants who received at least five of the seven sessions

**Participants who received fewer than five of the seven sessions

\section{Fidelity}

Table 3 shows that the mean duration of the sessions as reported by the facilitators was generally comparable with the time planned in the protocol. However, for session 5, facilitators reported spending on average 66 minutes while 75 minutes were planned. Table 3 further displays the extent to which the key components within each session of the AMB-Home program were performed according to protocol as reported by the facilitators. The key component, "discussion of a session's theme", was largely per- 
formed throughout all sessions. The performance of the component, "formulating an action plan", showed a decrease over time, indicating that toward the end of the program formulating an action plan was left out more often. Additionally, Table 3 shows that performance of a challenging activity by the participant under supervision of the facilitator in session 5 ("gradual exposure to an activity one is fearful of") was not performed by 34 participants (29\%).

Table 3 Fidelity: extent to which the key components of the AMB-Home program were performed according to protocol

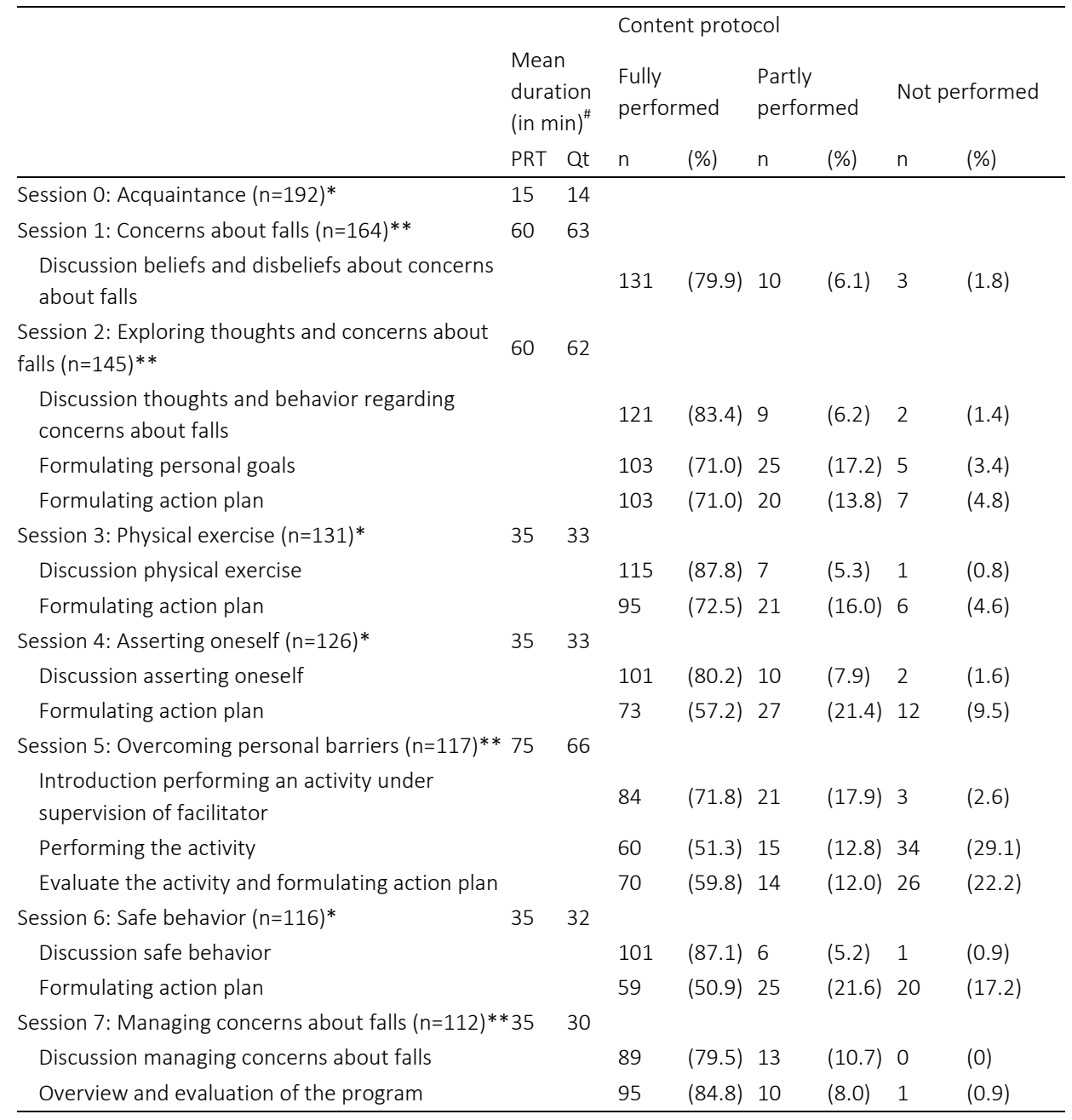

Note: all numbers and percentages may not add up to final numbers due to missing data.

\#PRT = duration according to the protocol; Qt = duration reported by the facilitator

*Telephone contact

**Home visit 
The facilitators' view of the extent to which the key components of the program were performed according to the protocol differed from that revealed in the 42 scored audio recordings. The facilitators reported that $81 \%$ of the components were fully performed, $6 \%$ were partly performed, and $7 \%$ were not performed, but in the audio recordings these percentages were $41 \%, 33 \%$, and $26 \%$, respectively. It should be noted that audio recordings from only six of the eight facilitators were available due to early withdrawal of two facilitators.

Facilitators reported they applied at least part of the techniques of behavior change with most participants, that is, delivering the essential information (89\%), allowing the participant to identify his/her important activities (84\%), letting the participant set goals regarding an action plan (86\%), and using motivational interviewing (87\%).

\section{Dose received - exposure}

With respect to engagement in the program, 103 of the 117 participants (88\%) rated their engagement as sufficient to very good; facilitators rated the engagement of 91 participants (78\%) as sufficient to very good. The adherence and quality of the homework was only rated by the facilitators; they reported that $78 \%$ of the participants did their homework assignments completely or to a great extent, and that the quality was sufficient to very good for almost $90 \%$ of the participants. The compliance of participants with the action plans was $74 \%$ according to the participants and $77 \%$ according to the facilitators. Facilitators were moderately positive about the extent to which participants could formulate and carry out action plans independently, reporting that $52 \%$ of the participants managed to do this completely or largely independently.

Furthermore, 88 participants (75\%) indicated that they reached at least one personal goal as formulated in session 2 (e.g., traveling independently by bus to the market in the city center), and 18 participants (15\%) mentioned that they did not achieve a personal goal or that no goals were formulated. Ten percent did not specify whether they had formulated a goal or not. Facilitators indicated that slightly fewer participants reached at least one goal $(n=82,70 \%)$.

The program materials developed for the participant were well applied; according to the participants $80 \%$ used the sheets for formulating action plans; $86 \%$ used the information sheets, for example on local welfare organizations; and $74 \%$ used the leaflets containing information about exercise and home safety.

For 28 participants (24\%), a significant other who could provide help and support to the participant during and after the program was present. A majority of these significant others were spouses $(n=23 ; 82 \%)$. According to the facilitators, the significant others actively supported the participants ( $n=26 ; 93 \%)$.

\section{Dose received - satisfaction}

Participants had a favorable opinion about the program while facilitators were significantly less positive (see Table 4). The facilitator's performance was highly valued by 
both the participants and the facilitators themselves. The support of the facilitator was the most frequently, spontaneously mentioned positive aspect of the program by the participants $(n=29)$ in the open-ended questions. Almost all participants $(97 \%)$ reported that they had understood the program. Similarly, facilitators reported that almost all participants (92\%) had at least sufficiently understood the program. Participants and facilitators also largely agreed on the benefits of the program for the participant's daily life: over $80 \%$ reported that participants had benefitted. The number and duration of the sessions were generally rated as sufficient. A preference for additional sessions was reported by six participants and one facilitator, who indicated that a participant would have benefitted from at least one extra session. According to the participants, the program was not overly burdensome (86\%), and they would recommend it to others (79\%).

Table 4 Dose received - satisfaction with the program: opinion of participant and facilitator

\begin{tabular}{|c|c|c|c|c|}
\hline & \multicolumn{2}{|c|}{$\begin{array}{l}\text { Participant } \\
(n=117)\end{array}$} & \multicolumn{2}{|c|}{$\begin{array}{l}\text { Facilitator }^{1} \\
(n=8)\end{array}$} \\
\hline & $\mathrm{n}$ & $(\%)$ & $\mathrm{n}$ & $(\%)$ \\
\hline \multicolumn{5}{|l|}{ the overall training } \\
\hline very good till sufficient & 106 & (90.6) & 86 & $(73.5)^{*}$ \\
\hline fair or poor & 7 & $(6.0)$ & 18 & $(15.4)$ \\
\hline \multicolumn{5}{|l|}{ the trainer's performance } \\
\hline very good till sufficient & 112 & $(95.7)$ & 108 & $(92.3)$ \\
\hline fair or poor & 1 & $(0.9)$ & 1 & $(0.9)$ \\
\hline \multicolumn{5}{|l|}{ comprehend the program } \\
\hline very good till sufficient & 113 & $(96.6)$ & 107 & $(91.5)^{*}$ \\
\hline fair or poor & 0 & $(0.0)$ & 4 & $(3.4)$ \\
\hline \multicolumn{5}{|l|}{ benefits in daily life } \\
\hline yes (definitely/somewhat) & 99 & $(84.6)$ & 94 & $(80.3)$ \\
\hline no (almost no/not) & 14 & $(12.0)$ & 18 & $(15.4)$ \\
\hline \multicolumn{5}{|l|}{ burdensome of the program } \\
\hline not burdensome & 101 & $(86.3)$ & & \\
\hline (too) burdensome & 12 & $(10.3)$ & & \\
\hline \multicolumn{5}{|c|}{ recommend the program to others } \\
\hline yes & 92 & $(78.6)$ & & \\
\hline no & 19 & $(16.2)$ & & \\
\hline
\end{tabular}

Note: all numbers and percentages may not add up to final numbers due to missing data.

*Significantly different with a Wilcoxon matched-pairs signed ranks test, $p<0.05$

${ }^{1}$ Facilitators evaluated the 117 participants who completed the program 
Participants and facilitators were questioned about potential benefits to the participant, of 11 topics addressed during the program (Table 5). 'Behaving more safely' was the topic most endorsed by both the participants and facilitators, and 'avoidance of activities' the least. Overall, were participants more optimistic than facilitators about the program benefits.

Table 5 Dose received - satisfaction: program benefits according to the participant and facilitator

\begin{tabular}{|c|c|c|c|c|}
\hline \multirow[b]{2}{*}{ Because of participating in the program ... } & \multicolumn{2}{|c|}{$\begin{array}{l}\text { Participant } \\
(\mathrm{n}=117)\end{array}$} & \multicolumn{2}{|c|}{$\begin{array}{l}\text { Facilitator } \\
(\mathrm{n}=8)^{1}\end{array}$} \\
\hline & $\mathrm{n}$ & $(\%)$ & $\mathrm{n}$ & $(\%)$ \\
\hline I behave more safely & 87 & $(74.4)$ & 78 & $(66.7)$ \\
\hline I make more optimal use of my opportunities & 86 & $(73.5)$ & 70 & $(59.8)^{*}$ \\
\hline My self-confidence has increased & 81 & $(69.2)$ & 65 & $(55.6)^{*}$ \\
\hline I became more physically active & 78 & $(66.7)$ & 71 & $(60.7)$ \\
\hline I am able to change negative thoughts into helpful thoughts & 76 & $(65.0)$ & 68 & $(58.1)$ \\
\hline I behave more assertively & 74 & $(63.2)$ & 50 & $(42.7)^{*}$ \\
\hline I am less concerned to fall & 70 & $(59.8)$ & 51 & $(43.6)^{*}$ \\
\hline I started new activities or took up old activities & 60 & $(51.3)$ & 67 & $(57.3)$ \\
\hline My risk of falling is reduced & 59 & $(50.4)$ & 54 & $(46.2)$ \\
\hline My home environment became safer & 55 & $(47.0)$ & 55 & $(47.0)$ \\
\hline I avoid fewer activities & 44 & $(37.6)$ & 43 & $(36.8)$ \\
\hline
\end{tabular}

Note: all numbers and percentages may not add up to final numbers due to missing data.

*Significantly different with a Wilcoxon matched-pairs signed ranks test, $p<0.05$

${ }^{1}$ Facilitators evaluated the 117 participants who completed the program

\section{Barriers and suggestions for improvement}

Barriers in applying the program

Facilitators indicated that more than one third of the 117 participants $(n=41)$ who completed the program should not have enrolled in the program. Reasons facilitators considered these participants not qualified were mainly: physical limitations $(n=9)$, not restricted in daily activities $(n=8)$, cognitive impairment $(n=5)$, not motivated for the program $(n=5)$, or not concerned about falling $(n=3)$. In the final group interview with the facilitators (If - see Table 1) facilitators mentioned that participants experienced difficulties in formulating sufficient goals for all action plans as planned in the program protocol, and in identifying a challenging activity of daily life to perform in session 5.

\section{Areas for improvement}

The main suggestions for improvement made by facilitators were changing the frequency of all sessions to weekly, shortening session 7 and adding a booster session after 3 months, selecting better qualified participants, and providing an internet forum for facilitators to share challenges and solutions in conducting the program. 
The majority of the participants $(n=63$ ) provided no suggestions for improvement. Of the 51 participants who suggested improvements the following were most often mentioned: reduce the repetition of parts of the program $(n=9)$, fewer or no telephone contacts (mainly mentioned with respect to hearing problems) $(n=8)$, simplify the program $(n=8)$, and offer more sessions $(n=5)$.

\section{Discussion}

This study was an examination of the feasibility and acceptability of a nurse-led in-home cognitive behavioral program (AMB-Home) to reduce concerns about falls and associated avoidance of activity in frail older people. Overall, both facilitators and participants considered the program acceptable and feasible. The program protocol was largely followed according to the facilitators. Participants' engagement in the program and satisfaction with the program was high and the perceived burden was low. Sixty percent of the participants received at least five of the seven sessions. The main reasons for not completing the program were lost interest and health problems. Suggestions for improvement of the program were provided by both facilitators and participants.

AMB-Home was developed to enable frail older people as well as people who prefer an (individual) in-home approach over a group approach to participate in a program to manage their concerns about falls. Comparing AMB-Home and AMB-NL, health problems were the main reason for withdrawal in $\mathrm{AMB}-\mathrm{NL}^{24}$, in contrast to the present study in which the most common reason was loss of interest. The difference in reasons for withdrawal reflects that fewer in-home participants experienced health problems as an obstacle to starting the program. A personal intake interview, to give tailor-made information about the program to match expectancies of potential participants with the actual content of the program and to check eligibility criteria in more detail, might be useful to further reduce withdrawal.

Based on one of the recommendations of the feasibility study of $\mathrm{AMB}-\mathrm{NL}^{24}$, more attention was paid to the homework assignments during the AMB-Home program. . The facilitators in this study reported that the extra attention resulted in a higher compliance of participants with the homework assignments and a better quality of the assignments than was reported by the facilitators of the group program. The individual approach might also have encouraged participants, because questions could be answered directly, and participants likely felt more obliged to do their homework because their assignment would be discussed at the next individual session.

The performance of a specific, challenging (fear-related) problem in daily life in the presence of the facilitator in session 5 was a new program component in AMB-Home compared to AMB-NL. Unfortunately, almost one third of the participants did not perform this gradual exposure to a fearful activity. According to the facilitators the main reason for not performing such an activity was the participant's inability to think of a 
suitable activity. It had been foreseen that selecting suitable activities for the goalsetting and action-planning components of the program could be challenging. ${ }^{35}$ To overcome this difficulty, 16 pictures of the Icon-FES ${ }^{36}$, which include the daily activities used in the assessment of concerns about falls by the FES-1 ${ }^{37,38}$, were incorporated in session 2 and served as examples for the selection of activities by the participant. To improve the uptake of this new part of the AMB-Home program, the training for facilitators might address the selection of suitable and relevant activities in more depth. Successful performed activities by participants in this study could serve as examples. It is, nevertheless, worthwhile to further explore ways to stimulate the performance of a specific, fear-related problem during the program sessions. In patients with specific phobias, exposure to fearful situations appears quite successful in reducing excessive fear and increasing functional abilities. ${ }^{39,40}$

A few limitations are worthy of discussion. First, both facilitators and participants may have given socially desirable answers. In order to reduce such bias, we explicitly told facilitators that their comments, recommendations and audio recordings would not be used for judging their professionalism but only for evaluation and improvement of the program. Participants were informed that the facilitators would not be informed about their individual answers and that their answers would not affect their (future) use of healthcare services.

Second, a difference was observed in adherence to the protocol according to the two different assessment methods. The self-reported adherence to the protocol by the facilitators was much higher than in the independent ratings of the audio recordings. An explanation for the difference between the self-report and the audio recordings could be that facilitators, despite our explicit statement that their answers would only be used for the process evaluation of this program, may have given socially desirable answers. Another explanation could be that facilitators were insufficiently aware of not following the protocol as intended. A third reason could be that facilitators had difficulty recalling the session when filling in the questionnaire afterward. However, when the answer categories 'fully performed' and 'partly performed' based on the audiotape scores were taken together, $74 \%$ of the program (compared to $88 \%$ in the facilitator self-reports) had been performed. Hence, the majority of participants were at least partly exposed to the content of the program. In the future, regular and individual feedback on facilitators' performance based on audio-taped sessions might increase treatment fidelity.

Last, one third of the participants, from the facilitators viewpoint, were not qualified to participate in the program. The reasons given for this impression were diverse. A personal intake, as mentioned before, could enable a better match between the program and qualified participants. However, there may be a certain gap between the participant's and the facilitator's perception eligibility.

Despite these limitations, the strengths of our study design included an extensive and mixed method data collection, including objective data in the form of audio record- 
ings, and a systematic approach which covered the key elements of reach, fidelity, dose received, and barriers.

AMB-Home was generally well performed by facilitators and well received by the frail older participants and therefore considered as feasible in the daily practice of nurses working in homecare organizations. Based on this study, the in-home program could be a good alternative in addition to the group program for particularly frail older people and those who prefer an individual in-home program. Yet, the program can be improved in some respects. First, an individual intake may improve enrollment in the program. This kind of intake procedure is currently successful applied in the Netherlands for the group program ${ }^{22}$; eligibility and motivation for the program is checked and clear information about the program to match expectancies is provided. Second, additional encouragement of participants to have a significant other co-participate in the program deserves further exploration. It is likely that a significant other can play a positive role in, for example, supporting the participant to formulate and implement action plans. Such encouragement may become a point of special interest in the communication with the participant prior to enrollment in the program. Third, a booster session by telephone after 3 months is recommended by the facilitators. A booster session is often recommended in self-management interventions to uphold the initial positive effects, yet, the effectiveness should be verified first. ${ }^{41}$

Last, adaptations will be needed when translating the program to other countries. Differences in healthcare systems and financial resources will guide the development, evaluation, implementation, and dissemination of programs. ${ }^{42}$ A Matter of Balance is a good example of how a program can be translated to many settings, for example, from the US to the Netherlands ${ }^{21-23}$, from leading by healthcare professionals to volunteer lay leaders $^{16}$, to different states in the US ${ }^{15,17,20}$; and from a group- to home-based program in this study. In addition, the possibility of integrating AMB-Home into other home programs can be studied.

Based on the results of this process study, we conclude that the program is feasible for frail community-dwelling older people with concerns about falls and related activity avoidance. The majority of the participants were satisfied with the program and benefitted from it, and the geriatric nurses who carried out AMB-Home were capable of conducting this cognitive behavioral program in home visits and telephone contacts. Potential improvements for the program include a personal intake to reduce early withdrawal and additional training for facilitators to bolster participants' selection of appropriate and realistic activities for the formulation and performance of action plans.

\section{Acknowledgements}

This research was funded by ZonMw, the Netherlands Organization for Health Research and Development (grant 120610001). The participation of author JV was supported by a 
grant from the Research Foundation, Flanders, Belgium (FWO Vlaanderen). Trial registration: NCT01358032. Preliminary outcomes were presented at the 64th and 65th Annual Scientific Meetings of the Gerontological Society of America in November 2011 and November 2012. We thank all participants, nurses, research assistants, and the Centre for Data and Information Management (MEMIC) for their assistance. 


\section{References}

1. Scheffer AC, Schuurmans MJ, van Dijk N, van der Hooft T, de Rooij SE: Fear of falling: measurement strategy, prevalence, risk factors and consequences among older persons. Age Ageing. 2008, 37(1):19-24.

2. Schepens S, Sen A, Painter JA, Murphy SL: Relationship between fall-related efficacy and activity engagement in community-dwelling older adults: a meta-analytic review. Am J Occup Ther. 2012, 66(2):137-148.

3. Fletcher PC, Guthrie DM, Berg K, Hirdes JP: Risk factors for restriction in activity associated with fear of falling among seniors within the community. J Patient Saf. 2010, 6(3):187-191.

4. Murphy SL, Williams CS, Gill TM: Characteristics associated with fear of falling and activity restriction in community-living older persons. J Am Geriatr Soc. 2002, 50(3):516-520.

5. Zijlstra GA, van Haastregt JC, van Eijk JT, van Rossum E, Stalenhoef PA, Kempen GI: Prevalence and correlates of fear of falling, and associated avoidance of activity in the general population of communityliving older people. Age Ageing. 2007, 36(3):304-309.

6. Yardley L, Smith H: A prospective study of the relationship between feared consequences of falling and avoidance of activity in community-living older people. Gerontologist. 2002, 42(1):17-23.

7. Friedman SM, Munoz B, West SK, Rubin GS, Fried LP: Falls and fear of falling: Which comes first? A longitudinal prediction model suggests strategies for primary and secondary prevention. J Am Geriatr Soc. 2002, 50(8):1329-1335.

8. Delbaere K, Close JC, Brodaty H, Sachdev P, Lord SR: Determinants of disparities between perceived and physiological risk of falling among elderly people: cohort study. BMJ. 2010, 341:c4165.

9. Arfken CL, Lach HW, Birge SJ, Miller JP: The prevalence and correlates of fear of falling in elderly persons living in the community. Am J Public Health. 1994, 84(4):565-570.

10. Cumming RG, Salkeld G, Thomas M, Szonyi G: Prospective study of the impact of fear of falling on activities of daily living, SF-36 scores, and nursing home admission. J Gerontol A Biol Sci Med Sci. 2000, 55(5):M299-305.

11. Delbaere K, Sturnieks DL, Crombez G, Lord SR: Concern about falls elicits changes in gait parameters in conditions of postural threat in older people. J Gerontol a-Biol. 2009, 64(2):237-242.

12. Howland J, Peterson EW, Levin WC, Fried L, Pordon D, Bak S: Fear of falling among the communitydwelling elderly. J Aging Health. 1993, 5(2):229-243.

13. Bula CJ, Monod S, Hoskovec C, Rochat S: Interventions aiming at balance confidence improvement in older adults: an updated review. Gerontology. 2011, 57(3):276-286.

14. Zijlstra GA, van Haastregt JC, van Rossum E, van Eijk JT, Yardley L, Kempen GI: Interventions to reduce fear of falling in community-living older people: a systematic review. J Am Geriatr Soc. 2007, 55(4):603-615.

15. Batra A, Melchior M, Seff L, Frederick N, Palmer RC: Evaluation of a community-based falls prevention program in South Florida, 2008-2009. Prev Chronic Dis. 2012, 9:E13.

16. Healy TC, Peng C, Haynes MS, McMahon EM, Botler JL, Gross L: The feasibility and effectiveness of translating a matter of balance into a volunteer lay leader model. J App/ Gerontol. 2008, 27(1):34-51.

17. Ory MG, Smith ML, Wade A, Mounce C, Wilson A, Parrish R: Implementing and disseminating an evidencebased program to prevent falls in older adults, Texas, 2007-2009. Prev Chronic Dis. 2010, 7(6):A130.

18. Smith ML, Jiang L, Ory MG: Falls efficacy among older adults enrolled in an evidence-based program to reduce fall-related risk: sustainability of individual benefits over time. Fam Community Health. 2012, 35(3):256-263.

19. Tennstedt S, Howland J, Lachman M, Peterson E, Kasten L, Jette A: A randomized, controlled trial of a group intervention to reduce fear of falling and associated activity restriction in older adults. J Gerontol B Psychol Sci Soc Sci. 1998, 53(6):P384-392.

20. Ullmann G, Williams HG, Plass CF: Dissemination of an evidence-based program to reduce fear of falling, South Carolina, 2006-2009. Prev Chronic Dis. 2012, 9:E103.

21. Zijlstra GA, van Haastregt JC, Ambergen T, van Rossum E, van Eijk JT, Tennstedt SL, Kempen GI: Effects of a multicomponent cognitive behavioral group intervention on fear of falling and activity avoidance in community-dwelling older adults: results of a randomized controlled trial. J Am Geriatr Soc. 2009, 57(11):2020-2028. 
22. Zijlstra GA, van Haastregt JC, Du Moulin MF, de Jonge MC, van der Poel A, Kempen Gl: Effects of the implementation of an evidence-based program to manage concerns about falls in older adults. Gerontologist. 2013, 53(5):839-849.

23. Zijlstra GA, Tennstedt SL, van Haastregt JC, van Eijk JT, Kempen GI: Reducing fear of falling and avoidance of activity in elderly persons: the development of a Dutch version of an American intervention. Patient Educ Couns. 2006, 62(2):220-227.

24. van Haastregt JC, Zijlstra GA, van Rossum E, van Eijk JT, de Witte LP, Kempen GI: Feasibility of a cognitive behavioural group intervention to reduce fear of falling and associated avoidance of activity in community-living older people: a process evaluation. BMC Health Serv Res. 2007, 7:156.

25. Dorresteijn TA, Rixt Zijlstra GA, Van Eijs YJ, Vlaeyen JW, Kempen GI: Older people's preferences regarding programme formats for managing concerns about falls. Age Ageing. 2012, 41(4):474-481.

26. Dorresteijn TA, Zijlstra GA, Delbaere K, van Rossum E, Vlaeyen JW, Kempen GI: Evaluating an in-home multicomponent cognitive behavioural programme to manage concerns about falls and associated activity avoidance in frail community-dwelling older people: Design of a randomised control trial [NCT01358032]. BMC Health Serv Res. 2011, 11:228.

27. Baranowski T, Stables G: Process evaluations of the 5-a-Day projects. Health Educ Behav. 2000, 27(2):157-166.

28. Linnan L, Steckler A: Process evaluation for public health interventions and research. An Overview. In: Process evaluation for public health interventions and research. Edited by Steckler A, Linnan L. San Francisco, CA: Jossey-Bass; 2002.

29. Oakley A, Strange V, Bonell C, Allen E, Stephenson J: Process evaluation in randomised controlled trials of complex interventions. BMJ. 2006, 332(7538):413-416.

30. Saunders RP, Evans MH, Joshi P: Developing a process-evaluation plan for assessing health promotion program implementation: a how-to guide. Health Promot Pract. 2005, 6(2):134-147.

31. Stewart AL, Hays RD, Ware JE, Jr.: The MOS short-form general health survey. Reliability and validity in a patient population. Med Care. 1988, 26(7):724-735.

32. van Sonsbeek JLA: Methodological aspects and content of the OECD-longterm disability indicator. CBS Maandberichten Gezondheid. 1988, 6:4-17.

33. Swain DG, Nightingale PG: Evaluation of a shortened version of the abbreviated mental test in a series of elderly patients. Clin Rehabil. 1997, 11(3):243-248.

34. Kempen GI, Meier AJ, Bouwens SF, van Deursen J, Verhey FR: [The psychometric properties of the Dutch version of the telephone interview cognitive status (TICS)]. Tijdschr Gerontol Geriatr. 2007, 38(1):38-45.

35. Daniels R: Frail elderly identification and disability prevention in primary care [PhD thesis]. Maastricht: Faculty of Health, Medicine and Life Sciences, University Maastricht; 2011.

36. Delbaere K, S TS, Lord SR: Development and initial validation of the iconographical falls efficacy scale. J Gerontol a-Biol. 2011, 66(6):674-680.

37. Kempen GI, Todd CJ, Van Haastregt JC, Zijlstra GA, Beyer N, Freiberger E, Hauer KA, Piot-Ziegler C, Yardley L: Cross-cultural validation of the falls efficacy scale international (FES-I) in older people: results from Germany, the Netherlands and the UK were satisfactory. Disabil Rehabil. 2007, 29(2):155-162.

38. Yardley L, Beyer N, Hauer K, Kempen G, Piot-Ziegler C, Todd C: Development and initial validation of the falls efficacy scale-international (FES-I). Age Ageing. 2005, 34(6):614-619.

39. Vlaeyen JWS, de Jong J, Geilen M, Heuts PHTG, van Breukelen G: The treatment of fear of movement/(re)injury in chronic low back pain: Further evidence on the effectiveness of exposure in vivo. Clin J Pain. 2002, 18(4):251-261.

40. Wolitzky-Taylor KB, Horowitz JD, Powers MB, Telch MJ: Psychological approaches in the treatment of specific phobias: a meta-analysis. Clin Psychol Rev. 2008, 28(6):1021-1037.

41. Kroese FM, Adriaanse MA, De Ridder DT: Boosters, anyone? Exploring the added value of booster sessions in a self-management intervention. Health Educ Res. 2012, 27(5):825-833.

42. Grol R, Grimshaw J: From best evidence to best practice: effective implementation of change in patients' care. Lancet. 2003, 362(9391):1225-1230. 


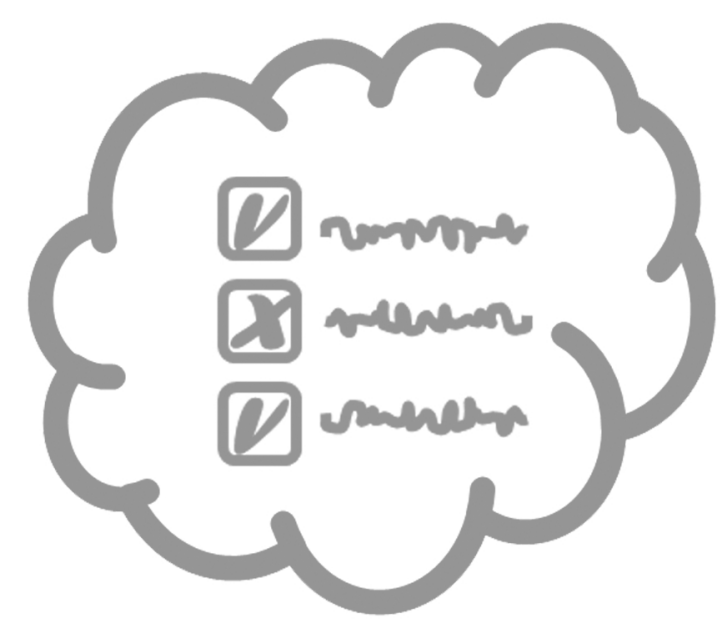




\section{Chapter 5}

Effectiveness of a home-based cognitive behavioral program to manage concerns about falls in community-dwelling, frail older people: results of a randomized controlled trial 


\begin{abstract}
Background Concerns about falls are common among older people. These concerns, also referred to as fear of falling, can have serious physical and psychosocial consequences, such as functional decline, increased risk of falls, activity restriction, and lower social participation. Although cognitive behavioral group programs to reduce concerns about falls are available, no home-based approaches for older people with health problems, who may not be able to attend such group programs are available yet. The aim of this study was to assess the effectiveness of a home-based cognitive behavioral program on concerns about falls, in frail, older people living in the community.
\end{abstract}

Methods In a randomized controlled trial in the Netherlands, 389 people aged 70 years and older, in fair or poor perceived health, who reported at least some concerns about falls and related activity avoidance were allocated to a control $(n=195)$ or intervention group ( $n=194)$. The intervention was a home-based, cognitive behavioral program consisting of seven sessions including three home visits and four telephone contacts. The program aims to instill adaptive and realistic views about fall risks via cognitive restructuring and to increase activity and safe behavior using goal setting and action planning and was facilitated by community nurses. Control group participants received usual care. Outcomes at 5 and 12 months follow-up were concerns about falls, activity avoidance due to concerns about falls, disability and falls.

Results At 12 months, the intervention group showed significant lower levels of concerns about falls compared to the control group. Furthermore, significant reductions in activity avoidance, disability and indoor falls were identified in the intervention group compared with the control group. Effect sizes were small to medium. No significant difference in total number of falls was noted between the groups.

Conclusions The home-based, cognitive behavioral program significantly reduces concerns about falls, related activity avoidance, disability and indoor falls in communityliving, frail older people. The program may prolong independent living and provides an alternative for those people who are not able or willing to attend group programs.

Trial registration NCT01358032 


\section{Background}

Concerns about falls, also referred to as fear of falling, are common among older people living in the community, with a prevalence of about 50 percent regardless of whether people experienced a recent fall. ${ }^{1,2}$ Negative consequences of concerns about falls include loss of balance confidence, social isolation, anxiety and symptoms of depression, avoidance of daily activities, physical frailty, falls, loss of independence, and institutionalization. ${ }^{1,3-9}$ It is therefore important for older people to manage their concerns about falls to maintain daily function and independence. Previous studies suggested that addressing factors in multiple domains may effectively reduce concerns about falls. ${ }^{10-13}$

'A Matter of Balance' (AMB) is a multicomponent, cognitive behavioral, community-based program to reduce concerns about falls. Matching one's activities to one's physical abilities is a main element in the intervention. Through realistic and adaptive appraisal, the intervention aims to reduce concerns about falls and related activity avoidance without increasing falls. Previous studies in community-dwelling older people showed the effectiveness of the program in reducing concerns about falls and associated activity avoidance without increasing actual falls. ${ }^{14}$ Additionally, the Dutch version of AMB (AMB-NL) demonstrated a reduction in the restriction of daily living activities and recurrent fallers. ${ }^{15}$ The program uses cognitive restructuring and behavioral change techniques to address psychosocial (e.g., beliefs about falls and fall risk, social support, and assertiveness), physical (e.g., participation in physical activities and vision screening) and functional (e.g., safe behavior, participating in and continuing daily life activities) factors related to concerns about falls. AMB was originally developed as a groupbased program, which can be delivered by trained healthcare professionals or volunteers. After evaluation research, the group program has been successfully implemented in different settings, versions and countries. ${ }^{16-21}$ Despite the success of AMB, not all eligible older people participate. Especially those with health problems tend to withdraw prior and during the group program. ${ }^{14,22}$ In addition, not all older people prefer to participate in a group approach ${ }^{23}$ To allow particularly frail older people to participate in the program and to benefit from its effects an individualized, home-based format of AMB-NL additional to the group approach was therefore developed. ${ }^{24}$ The home-based format of 'A Matter of Balance' (AMB-Home) includes three home visits and four telephone contacts and aims to encourage independent living among older people for as long as possible with minimal burden for healthcare professionals and informal caregivers. ${ }^{25-27}$

The current paper reports on the results of a randomized controlled trial to evaluate the effects of AMB-Home compared with usual care on concerns about falls in community-dwelling, frail older people. Secondary outcomes of the trial were avoidance of activity due to concerns about falls, disability, and indoor and outdoor falls. 


\section{Methods}

\section{Study Design}

In this two-group randomized controlled trial (RCT), community-dwelling older people were selected in four consecutive cycles in 2009. In the Netherlands all citizens are registered in municipal registry offices and to select a representative sample, addresses of potential participants were randomly drawn by three offices in the south of the country. All cycles started between March and December 2009, and each cycle lasted 15 months. A cycle included screening for eligible participants, baseline measurements, stratified randomization, an intervention period of approximately 4 months, and two follow-up measurements at 5 and 12 months. To screen for eligibility, people received a short postal questionnaire with a freepost envelope, as well as information about the trial and an informed consent form. The Medical Ethics Committee of the Maastricht University/Academic Hospital Maastricht in the Netherlands approved the study (MEC 07-3-064). The trial was performed as planned. Additional information about the study design can be found in a published study protocol. ${ }^{24}$

\section{Participants}

Community-dwelling people aged 70 years or older were included in the study if they reported at least some concerns about falls and associated activity avoidance, perceived their general health as fair or poor, and were willing to participate (signed informed consent form) (see Table 1). The criteria with respect to concerns about falls and associated activity avoidance were based on two items: 1) "Are you concerned about falling?" and 2) "Do you avoid certain activities due to concerns about falling?" Answer options for both items included 'never', 'almost never', 'sometimes', 'regularly', 'often', and 'very often'. We included individuals with answers ranging from 'sometimes' to 'very often' regarding both concern about falling and activity avoidance. In this study, we used the term "frail" in relation to our sample to indicate that our sample perceived their general health as fair or poor in conjunction with reported concerns about falls and related activity avoidance. As a result, we could make clear that we included those participants who were unlikely to participate in a group program because of health problems. Individuals were excluded if they were confined to bed; wheelchair dependent; waiting for nursing home admission; or experienced substantial hearing, vision or cognitive impairments. All inclusion and exclusion criteria were assessed during the screening, with the exception of cognitive impairment, which was assessed during the baseline measurement using the 4-item Abbreviated Mental Test (AMT4). ${ }^{28}$ If individuals scored < 4 on the AMT4, the Telephone Interview Cognitive Status (TICS) was administered. Individuals were excluded if they scored < 17 out of 41 on the TICS. ${ }^{29}$ Additionally, a restriction was applied to couples; only one member of a couple was allowed to participate in the trial to prevent reciprocal influencing if by chance one was allocated to the treatment group and one to the control group. Lots were drawn to determine who of the couple would be included. 


\section{Randomization}

To prevent an imbalance between groups, stratified randomization was used to randomly allocate participants to either the control group or the intervention group. A computerized two-block randomization was performed using the level of concerns about falls (i.e., sometimes, regular, often, and very often) as the prognostic factor. An external agency blinded to participant characteristics conducted the randomization directly after the baseline measurement. Cross-over between groups was not permitted, and participants were aware of their group allocation.

\section{Intervention}

The purpose of our individual, home-based, cognitive behavioral AMB-Home program was to shift maladaptive to adaptive cognitions with respect to falling and concerns about falls. The program aims to instill a realistic view of fall risk, increasing self-efficacy beliefs and feelings of control, and changing behavior. To achieve these goals, the following strategies were applied: 1) identifying and restructuring misconceptions about falls and fall risk; 2) setting realistic personal goals for increasing activity levels and safe behavior; and 3) promoting the uptake of old and new daily life activities that were avoided due to concerns about falls.

The AMB-Home program consists of seven individual sessions, including three home-visits (60, 60 and 75 minutes, respectively) and four telephone contacts (35 minutes each). The seven pre-defined themes of the program were concerns about falls; thoughts about falling; physical exercise; asserting oneself; overcoming personal barriers; safe behavior; and managing concerns about falls. ${ }^{24}$ Each session was similarly structured with a review of the previous session (except the first session), a discussion of the main theme, and the formulation of a personalized action plan related to the discussed theme. Session 5 differed slightly from the other sessions in that participants were guided to safely execute a daily activity they were afraid to perform independently ('exposure in vivo'). ${ }^{30}$ Examples of activities selected by participants included walking down the stairs or crossing a street. The participants received homework assignments between the sessions, including reading informative leaflets, filling in checklists to become aware of their beliefs about falls, and executing personal action plans. In addition, a DVD was used to show how peers address concerns about falls.

AMB-Home includes detailed manuals for both the participants and the program facilitators. The facilitators were community nurses $(n=8)$ who were qualified in the field of geriatrics and worked at local home-care agencies. Prior to the start of the trial, the nurses received a two-day, mandatory training. During this training, the nurses became familiar with the content of the program and behavior change techniques. Professionals with expertise in motivational interviewing, behavioral change, and 'exposure in vivo' contributed to the training program.

Overall, principles for behavior change and themes of the group program were maintained in AMB-Home. However, in adapting the group program to a home-based 
program several changes were made. First, the physical exercises in the group program were replaced by 'exposure in vivo' ${ }^{30}$, because the appropriate and safe execution of these exercises could not be guaranteed due to the limited face-to-face contact. Second, motivational interviewing was incorporated to encourage internal motivation to change behavior and increase self-efficacy. ${ }^{31}$ Next, participants were encouraged to invite a significant other (e.g., a spouse, friend, or neighbor) to be present during the home visits. This person could motivate the participant to perform the action plans between the sessions. Lastly, the eight group sessions (120 minutes each) were replaced by seven individual sessions, including three home-visits $(60,60$ and 75 minutes, respectively) and four telephone contacts (35 minutes each). We considered that modeling and vicarious experiences are active ingredients of self-efficacy theory and are despite the use of the DVD less pronounced in the home-based program, individual support from the facilitator by action planning is more dominant in AMB-Home as well as the potential impact of a significant other. An overview of AMB-Home and the differences with AMB-NL are described elsewhere. ${ }^{24}$

The control group received care as usual. Whereas no standard treatment for concerns about falls was available during the study period it is likely they received no treatment.

\section{Outcome Measurements}

With the exception of the registration of falls using a monthly calendar, data were collected at baseline and at 5- and 12-month follow-up via telephone interviews. Facilitators and participants were aware of group assignments; outcome assessors were blinded to the allocation. Prior to data collection, the outcome assessors from a center for data and information management participated in a two-hour training session on assessment procedures and study design.

The primary outcome was concerns about falls measured with the 16-item Falls Efficacy Scale-International (FES-I; range 16 to 64). This scale assesses an individual's level of concerns about falls while performing activities of daily living (ranging from $1=$ not at all concerned to 4 = very concerned). ${ }^{32,33}$

Secondary outcomes included avoidance of activity as a result of concerns about falls, disability, number of falls, and medical attention received after a fall incident. Avoidance of activity was measured using a modified version of the 16 -item FES-I. If participants indicated that they experienced at least some concerns about falls while performing a certain activity, they were asked to indicate to what extent they avoided that activity as a result of their concerns (Falls Efficacy Scale-International Avoidance Behavior (FES-IAB); 1 = never and 4 = often; range 16 to 64$).{ }^{24,34}$ Disability was measured using the 18-item Groningen Activity Restriction Scale (GARS). The GARS assesses the extent to which individuals have difficulty in performing 18 activities of daily living (ranging from 1 = yes, can do fully independently to $4=$ no, can do only with help from others; range 18 to 72$).{ }^{35}$ Both disabilities in the areas of Activities of Daily Living, including mobility, (ADL; 11 items; range 11 tot 44) and Instrumental Activities of Daily 
Living (IADL; 7 items; range 7 to 28 ) are embedded in the GARS. Falls were assessed using a monthly calendar that indicated whether a fall occurred in the past week. A fall was defined as an event that results in a person coming to rest inadvertently on the ground or on another lower level. ${ }^{36}$ If a fall occurred, participants reported the location of the fall (indoor or outdoor) and the number of times medical attention related to the fall was received. Participants returned the calendar sheets each month and were reminded by telephone if a calendar was not returned after 10 days.

Demographic characteristics including age, gender, living situation, educational level (low: completed elementary school; middle: completed secondary school; high: completed higher vocational training or university level ${ }^{37}$ ), perceived general health, falls in the past 6 months, and active chronic diseases (i.e., diseases for which a physician was consulted or medicines were administered in the previous 12 months) were collected.

\section{Sample size}

To detect a mean difference of at least 3.8 points (effect size of .33 on the FES-I) between the intervention and control group, 112 participants per group were required to provide $80 \%$ power at alpha .05 (one-tailed). These sample size calculations were based on outcomes of a previous study using the FES-I among older people in the Netherlands. ${ }^{33}$ However, we expected a $20 \%$ dropout rate during the current study; therefore at least $280(2 \times 140)$ participants were needed for the final analyses in this trial. ${ }^{24}$

\section{Statistical analyses}

Descriptive techniques were used for the variables of interest. Data were analyzed according to the intention-to-treat principle; therefore, all participants were included based on their original allocation. Missing values were imputed at the level of the scale by means of multiple imputations. The maximum number of missing values within a scale was based on guidelines provided by the developers. A limit of $25 \%$ missing values was used if no guidelines were available. Because multilevel analyses are quite robust against missing values at the measurement level, only the baseline measurement and one of the two follow-up measurements were needed to include participants in the analyses. The number of fallers, falls and fall-related medical attention were analyzed with negative binomial regression models and logistic regression models. All other outcomes were assessed using mixed-effects linear regression analyses. Models were adjusted for the stratification factor (i.e., concerns about falls), the baseline value of the outcome measure, age, gender, perceived general health, and number of falls in the 6 months before baseline. These covariates were considered a priori as relevant to the outcomes based on the literature. ${ }^{24}$ The interaction term group $X$ time was added to the model to determine the effects of the intervention (i.e., differences between the intervention and control group) at the two follow-up measurements. The level of statistical significance was set at .05 for those intervention effects where we expected an improvement in function (one-tailed), i.e., concerns about falls, avoidance of activities, 
and disability. ${ }^{24}$ For the baseline characteristics and fall data, the level of statistical significance was set at .05 (two-tailed). The results are presented with adjusted mean differences, odds ratios (ORs) or incidence rate ratios (IRRs), 95\% confidence intervals (Cls) and, if applicable for significant differences, effect sizes. Effect sizes of .20 are considered small, .50 medium, and .80 large. $^{38}$

For the primary outcome the Reliable Change Index (RCl) score was computed to determine whether the change score (12-month follow-up score minus baseline score) of a participant lies outside the range of $95 \%$ central change scores expected in case of no effect. The formula for $\mathrm{RCl}$ is change score divided by $\mathrm{SE}_{\text {diff, }}$ where $\mathrm{x}_{1}$ represents a participant's pretest score, $x_{2}$ represents that same participant's posttest score, $\mathrm{SD}_{1}$ is the standard deviation of the baseline scores and, $r$ is the reliability of the measurement.

$$
\mathrm{RCl}=\frac{x_{2}-x_{1}}{\mathrm{SE}_{\mathrm{diff}}} \quad \text { and } \mathrm{SE}_{\text {diff }}=\mathrm{SD}_{1} \mathrm{~V} 2 \mathrm{~V}(1-r)
$$

The $\mathrm{RCl}$ is then compared with $+/-1.96$. The formula change score $+/-1.96 * \mathrm{SE}_{\text {diff }}$ was used for a 95\% confidence interval for the true change scores. 39,40

Furthermore, pre-planned per protocol analyses were performed; outcomes of participants in the control group were compared with those of intervention participants who received less than five sessions and intervention participants who received at least five of the seven program sessions. ${ }^{24}$ Based on prior work, five sessions of the program were considered to be sufficient program exposure. ${ }^{14,15}$ All analyses were performed in SPSS 21.0.1 (SPSS, Inc., Chicago, IL).

\section{Results}

\section{Participants}

The flow of participants during the trial is presented in Figure 1. Eligibility screening occurred in the general community (see Study Design). Through randomization, 195 participants were allocated to the control group, and 194 participants were included in the intervention group. Baseline characteristics were comparable in both groups (Table 1). The dropout rates during the trial were $17 \%(n=33)$ in the control and $31 \%(n=61)$ in the intervention group. Withdrawal was highest at the 5-month follow-up measurement, which was directly after the intervention period. The main reasons for lost to follow-up were similar in the control and intervention group, i.e., lost interest and health problems. No significant differences were identified regarding baseline characteristics and primary and secondary outcomes between dropouts in the intervention and control groups (not tabulated). Thirty participants in the intervention group (15\%) were not exposed to the program; they withdrew from the intervention prior to the first session. Among the people who started the program, 29\% (47 out of 164) withdrew. Main reasons for not starting or withdrawal during the program were lost interest $(n=27)$, 
health problems $(n=16)$, and perceived burden $(n=8)$. Participants who received at least five of the seven sessions, rated their program engagement and satisfaction as high, and the burden of the program as low. More detailed information about the reach, fidelity, exposure, satisfaction and barriers of the program is published elsewhere. ${ }^{41}$

Table 1 Baseline characteristics of participants $(N=389)$

\begin{tabular}{|c|c|c|c|c|c|}
\hline & \multicolumn{2}{|c|}{$\begin{array}{l}\text { Control group } \\
(n=195)\end{array}$} & \multicolumn{2}{|c|}{$\begin{array}{l}\text { Intervention group } \\
(n=194)\end{array}$} & \multirow[t]{2}{*}{$p$-value } \\
\hline \multicolumn{5}{|l|}{ Demographic } & \\
\hline Mean age in years (SD) & 78.25 & $(5.3)$ & 78.38 & $(5.4)$ & 0.81 \\
\hline Gender (\%) & & & & & 0.36 \\
\hline Male & 54 & $(27.7)$ & 62 & $(32.0 \%)$ & \\
\hline Female & 141 & $(72.3)$ & 132 & $(68.0)$ & \\
\hline Living situation (\%) & & & & & 0.45 \\
\hline Not alone & 77 & (39.5) & 84 & $(43.3)$ & \\
\hline Alone & 118 & $(60.5)$ & 110 & $(56.7)$ & \\
\hline Educational level (\%) & & & & & 0.20 \\
\hline Low & 100 & $(51.5)$ & 110 & $(57.3)$ & \\
\hline Middle & 72 & $(37.1)$ & 55 & $(28.6)$ & \\
\hline High & 22 & $(11.3)$ & 27 & $(14.1)$ & \\
\hline \multicolumn{6}{|l|}{$\underline{\text { Health-related }}$} \\
\hline Perceived general health (\%) & & & & & 0.16 \\
\hline Fair & 176 & $(90.3)$ & 166 & $(85.6)$ & \\
\hline Poor & 19 & $(9.7)$ & 28 & $(14.4)$ & \\
\hline Mean number of active chronic diseases (SD) & 1.62 & $(1.0)$ & 1.57 & $(1.0)$ & 0.66 \\
\hline \multicolumn{6}{|l|}{$\underline{\text { Fall-related }}$} \\
\hline Falls in the past 6 months (\%) & & & & & 0.11 \\
\hline Never & 81 & $(42.2)$ & 64 & $(33.3)$ & \\
\hline Once & 55 & $(28.6)$ & 54 & $(28.1)$ & \\
\hline More than once & 56 & $(29.2)$ & 74 & $(38.5)$ & \\
\hline Concerns about falls (\%) & & & & & 1.00 \\
\hline Sometimes & 90 & $(46.2)$ & 91 & $(46.9)$ & \\
\hline Regular & 54 & $(27.7)$ & 53 & $(27.3)$ & \\
\hline Often & 32 & $(16.4)$ & 31 & $(16.0)$ & \\
\hline Very often & 19 & $(9.7)$ & 19 & $(9.8)$ & \\
\hline Avoidance of activities (\%) & & & & & 0.29 \\
\hline Sometimes & 104 & $(53.3)$ & 85 & $(43.8)$ & \\
\hline Regular & 50 & $(25.6)$ & 62 & $(32.0)$ & \\
\hline Often & 25 & $(12.8)$ & 27 & $(13.9)$ & \\
\hline Very often & 16 & $(8.2)$ & 20 & $(10.3)$ & \\
\hline
\end{tabular}

Note: all numbers and percentages may not add up to final numbers due to missing data. 


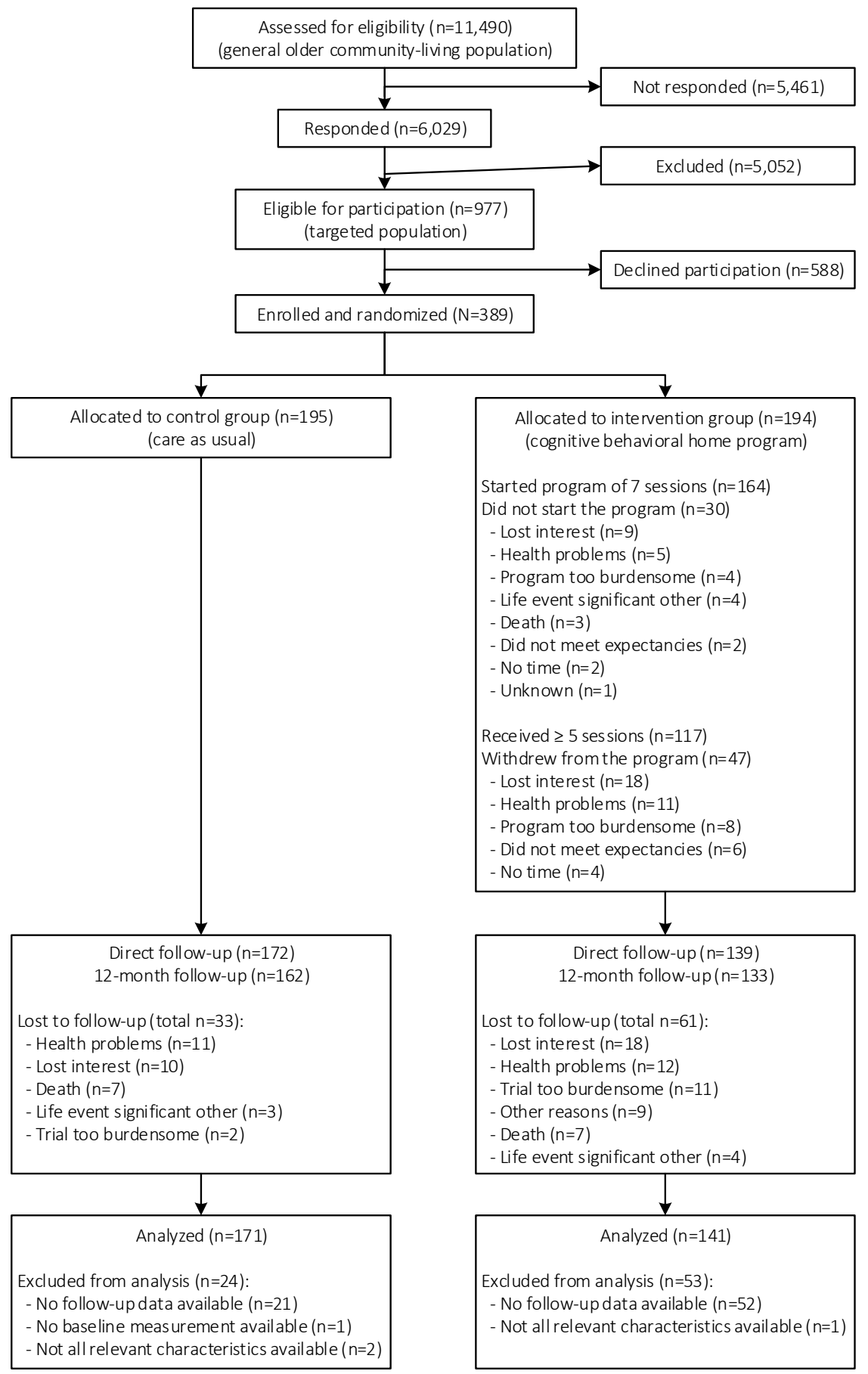

Figure 1 flow of participants through the trial 


\section{Outcomes}

Table 2 indicates that the intervention significantly reduced concerns about falls at both the 5-month (adjusted mean difference $=-3.53 ; P<.001$ ) and 12 -month follow-up (adjusted mean difference $=-3.92 ; P<.001)$.

Table 2 Effects of the home-based cognitive behavioral program on primary outcome ${ }^{\text {a }}$

\begin{tabular}{|c|c|c|c|c|c|c|c|}
\hline & \multicolumn{2}{|c|}{$\begin{array}{l}\text { Control group } \\
n=171\end{array}$} & \multicolumn{2}{|c|}{$\begin{array}{l}\text { Intervention } \\
\text { group } \\
n=141\end{array}$} & Model $^{\mathrm{b}}$ & \multirow[t]{2}{*}{$\begin{array}{l}\mathrm{P} \text { - } \\
\text { value }\end{array}$} & $\begin{array}{l}\text { Effect } \\
\text { size }\end{array}$ \\
\hline & Mean & (SD) & Mean & (SD) & $\begin{array}{l}\text { Adjusted mean } \\
\text { difference }(95 \% \mathrm{Cl})\end{array}$ & & $d$ \\
\hline \multicolumn{8}{|l|}{ FES-I } \\
\hline Baseline & 35.47 & $(9.4)$ & 35.70 & $(10.4)$ & - & - & - \\
\hline 5-month follow-up & 35.30 & $(10.4)$ & 31.73 & $(10.4)$ & $-3.53(-\infty--2.15)$ & $<.001$ & .34 \\
\hline 12-month follow-up & 35.86 & $(11.1)$ & 31.98 & (10.9) & $-3.92(-\infty--2.52)$ & $<.001$ & .35 \\
\hline
\end{tabular}

Note: FES-I= Falls Efficacy Scale-International (range total scale 16-64; higher scores indicate more concerns about falls).

${ }^{a}$ Results of mixed-effects linear regression analyses (intention-to-treat).

${ }^{b}$ Adjusted for baseline score of the outcome and level of concerns about falls, age, gender, perceived general health, and falls in the past 6 months.

$95 \% \mathrm{Cl}=95 \%$ confidence interval (one-sided); SD = standard deviation; $\infty=$ infinity.

Improvement was also observed for the secondary outcomes (Table 3), with the exception of IADL at the 5-month follow-up (adjusted mean difference $=-0.52 ; P=.075$ ). The effect sizes for significant differences were generally small to medium (.10 to .35 ).

Table 3 Effects of the home-based cognitive behavioral program on secondary outcomes ${ }^{a}$

\begin{tabular}{|c|c|c|c|c|c|c|c|}
\hline & $\begin{array}{l}\text { Contro } \\
\mathrm{n}=171\end{array}$ & group & $\begin{array}{l}\text { Interve } \\
\text { group } \\
n=141\end{array}$ & ntion & Model $^{b}$ & $\begin{array}{l}\mathrm{P}- \\
\text { value }\end{array}$ & $\begin{array}{l}\text { Effect } \\
\text { size }\end{array}$ \\
\hline & Mean & (SD) & Mean & (SD) & $\begin{array}{l}\text { Adjusted mean } \\
\text { difference }(95 \% \mathrm{Cl})\end{array}$ & $P$ & $d$ \\
\hline \multicolumn{8}{|l|}{$F E S-I A B$} \\
\hline Baseline & 29.09 & (9.3) & 29.06 & $(9.7)$ & - & - & - \\
\hline 5-month follow-up & 28.74 & $(9.2)$ & 26.17 & $(9.6)$ & $-2.38(-\infty--1.12)$ & .001 & .27 \\
\hline 12-month follow-up & 29.36 & $(10.3)$ & 26.37 & $(10.4)$ & $-2.67(-\infty--1.37)$ & .001 & .29 \\
\hline \multicolumn{8}{|l|}{ GARS } \\
\hline Baseline & 33.73 & (9.3) & 34.11 & $(9.4)$ & - & - & - \\
\hline 5-month follow-up & 33.32 & (8.9) & 32.42 & (8.9) & $-1.10(-\infty--0.07)$ & .040 & .10 \\
\hline 12-month follow-up & 34.04 & $(9.3)$ & 32.41 & $(9.4)$ & $-1.81(-\infty--0.77)$ & .002 & .17 \\
\hline
\end{tabular}




\begin{tabular}{|c|c|c|c|c|c|c|c|}
\hline & $\begin{array}{l}\text { Contro } \\
n=171\end{array}$ & group & $\begin{array}{l}\text { Interve } \\
\text { group } \\
\mathrm{n}=141\end{array}$ & ntion & Model $^{b}$ & $\begin{array}{l}\mathrm{P} \text { - } \\
\text { value }\end{array}$ & $\begin{array}{l}\text { Effect } \\
\text { size }\end{array}$ \\
\hline & Mean & $(S D)$ & Mean & (SD) & $\begin{array}{l}\text { Adjusted mean } \\
\text { difference }(95 \% \mathrm{Cl})\end{array}$ & $P$ & $d$ \\
\hline \multicolumn{8}{|l|}{ GARS ADL } \\
\hline Baseline & 18.70 & $(4.9)$ & 18.47 & (4.9) & - & - & - \\
\hline 5-month follow-up & 18.28 & $(4.5)$ & 17.37 & (4.7) & $-0.62(-\infty--0.04)$ & .039 & .20 \\
\hline 12-month follow-up & 18.69 & $(4.8)$ & 17.60 & (4.9) & $-0.83(-\infty--0.24)$ & .011 & .22 \\
\hline \multicolumn{8}{|l|}{ GARS IADL } \\
\hline Baseline & 15.03 & $(4.9)$ & 15.64 & (5.1) & - & - & - \\
\hline 5-month follow-up & 15.05 & $(5.1)$ & 15.04 & $(4.8)$ & $-0.52(-\infty-0.08)$ & .075 & - \\
\hline 12-month follow-up & 15.35 & $(5.1)$ & 14.82 & $(5.0)$ & $-1.01(-\infty--0.41)$ & .003 & .10 \\
\hline
\end{tabular}

Note: FES-IAB = Falls Efficacy Scale-International Avoidance Behavior (range total scale 16-64; higher scores indicate more activity avoidance due to concerns about falls).

GARS = Groningen Activity Restriction Scale (range total scale 18-72; higher scores indicate more disability); GARS ADL = Groningen Activity Restriction Scale - activities of daily living (ADL) subscale (range total scale 11-44; higher scores indicate more disability); GARS IADL = Groningen Activity Restriction Scale-instrumental ADL subscale (range total scale 7-28; higher scores indicate more disability).

${ }^{a}$ Results of mixed-effects linear regression analyses (intention-to-treat).

${ }^{b}$ Adjusted for baseline score of the outcome and level of concerns about falls, age, gender, perceived general health, and falls in the past 6 months.

$95 \% \mathrm{Cl}=95 \%$ confidence interval (one-sided); SD = standard deviation; $\infty=$ infinity.

Table 4 shows the outcomes with respect to reliable change in concerns about falls. The $\mathrm{SE}_{\text {diff }}$ of the FES-I was 4.2. This means that the $95 \%$ confidence interval of expected differences in case of no effect was between 8.2 and -8.2 points. Therefore, a reliable improvement was defined as an improvement of at least 9 points on the FES-I between baseline and 12-month follow-up and a reliable deterioration was defined as a decrease of at least 9 points in this timeframe. Based on a $\mathrm{RCl}$ score of 1.96 or higher, 30 participants $(22.6 \%)$ improved in the intervention group versus $14(8.7 \%)$ in the control group. In addition, 23 participants (14.3\%) deteriorated in the control group versus $9(6.8 \%)$ in the intervention group ( $\mathrm{RCl}$ score of -1.96 or lower).

Table 4 Reliable Change Index (RCI) of Concerns about Falls

\begin{tabular}{lllll}
\hline & $\begin{array}{l}\text { Control group } \\
\mathrm{n}=161\end{array}$ & \multicolumn{3}{l}{$\begin{array}{l}\text { Intervention group } \\
\mathrm{n}=133\end{array}$} \\
\hline Concerns about Falls (FES-I) & $\mathrm{n}$ & $(\%)$ & $\mathrm{n}$ & $(\%)$ \\
\hline Reliable deterioration $^{\mathrm{a}}$ & 23 & $(14.3)$ & 9 & $(6.8)$ \\
Not improved & 124 & $(77.0)$ & 94 & $(70.7)$ \\
Reliable improved $^{\mathrm{b}}$ & 14 & $(8.7)$ & 30 & $(22.6)$ \\
\hline
\end{tabular}


Note: Concerns about falls is measured with the FES-I (range total scale 16-64; higher scores indicate more concerns about falls). The FES-I reliable change index (RCI) score is calculated according to the outcomes on baseline and 12-month follow-up.

${ }^{\mathrm{a}} \mathrm{RCl}$ score 1.96 or higher (equal to a FES-I score difference of 9 or higher)

${ }^{b} \mathrm{RCl}$ score -1.96 or lower (equal to a FES-I score difference of -9 or lower).

The effects of the intervention on falls are presented in Table 5. Regarding the total number of falls, the number of outdoor falls and the number of times medical attention was required no significant differences were identified between the groups. Significantly fewer indoor falls were observed in the intervention group $(\mathrm{IRR}=0.68 ; P<.014)$.

In contrast to the intention-to-treat analyses, a significant difference was identified in the per-protocol analyses for IADL at 5-months for those who participated in at least five sessions compared with the control group (adjusted mean difference $=-0.64 ; P<$ .050). The effect sizes for the other outcomes (i.e., concerns about falls, related activity avoidance, and falls) remained similar (data not shown).

Table 5 Effects of the Home-Based Cognitive Behavioral Program on Fall Outcomes

\begin{tabular}{|c|c|c|c|c|c|c|}
\hline & \multicolumn{2}{|c|}{$\begin{array}{l}\text { Control group } \\
n=180\end{array}$} & \multicolumn{2}{|c|}{$\begin{array}{l}\text { Intervention group } \\
\mathrm{n}=166\end{array}$} & \multirow{2}{*}{$\begin{array}{l}\text { Model }^{\mathrm{a}} \\
\text { OR }(95 \% \mathrm{Cl})\end{array}$} & \multirow{2}{*}{$\frac{P \text {-value }}{P}$} \\
\hline & $\mathrm{n}$ & $(\%)$ & $\mathrm{n}$ & $(\%)$ & & \\
\hline \multicolumn{7}{|l|}{ Fallers } \\
\hline $\begin{array}{l}\text { Baseline until 12-month } \\
\text { follow-up }\end{array}$ & 106 & $(58.9)$ & 94 & $(56.6)$ & $0.79(0.50-1.23)$ & .292 \\
\hline \multicolumn{7}{|l|}{ Recurrent fallers } \\
\hline \multirow[t]{2}{*}{$\begin{array}{l}\text { Baseline until 12-month } \\
\text { follow-up }\end{array}$} & 67 & $(37.2)$ & 55 & (33.1) & $0.67(0.41-1.09)$ & .104 \\
\hline & \multicolumn{2}{|c|}{ Number ${ }^{b}$} & \multicolumn{2}{|c|}{ Number ${ }^{b}$} & $\operatorname{IRR}(95 \% \mathrm{CI})$ & $P$ \\
\hline Total falls & \multicolumn{2}{|l|}{429} & \multicolumn{2}{|c|}{362} & $0.86(0.65-1.13)$ & .273 \\
\hline indoor falls & \multicolumn{2}{|l|}{291} & \multicolumn{2}{|c|}{202} & $0.68(0.50-0.92)$ & .014 \\
\hline outdoor & \multicolumn{2}{|l|}{138} & \multicolumn{2}{|c|}{160} & $1.11(0.78-1.56)$ & .568 \\
\hline $\begin{array}{l}\text { No. of times medical } \\
\text { attention required as a } \\
\text { result of falls }\end{array}$ & \multicolumn{2}{|l|}{87} & \multicolumn{2}{|c|}{106} & $1.42(0.96-2.10)$ & .083 \\
\hline
\end{tabular}

Note: Results of mixed-effects logistic and negative binomial regression analyses

${ }^{a}$ Model adjusted for baseline score measurement and level of concerns about falls, age, gender, perceived general health, and falls in the past 6 months

${ }^{b}$ Analyses were performed with a Poisson distribution. This distribution of fall events accounts for over dispersion and incorporates both number of falls and time (weeks) of follow-up; herefore, all available data was used.

95\% Cl = 95\% confidence interval; OR = odds ratio mixed-effects logistics regression; IRR = incidence rate ratio obtained via negative binomial regression. 


\section{Discussion}

In this RCT the AMB-Home program significantly reduced concerns about falls in community-living, frail older people for up to 12 months. The home-based, cognitive behavioral program also showed favorable effects regarding the reduction of avoidance of activity due to concerns about falls, disability, and the number of indoor falls in the intervention group compared with the control group. No significant difference was found for total falls.

The outcomes of our study add to the increasing evidence that 'A Matter of Balance' as a cognitive behavioral approach positively influences concerns about falls and related avoidance behavior in older people. The setting and format in which the program is performed hardly affect the outcomes of the program, at least in the US and Western Europe. ${ }^{14,15,17,18,20,21}$ This facilitates the use of the program as it can be tailored to the preferences and abilities of older people. ${ }^{23}$

Strengths of this study include a solid methodological design with a one year follow up period for the effect evaluation and a comprehensive process evaluation during the intervention period ${ }^{24}$. Recruitment of participants went as planned; a sample of frail people was selected from the general older population living in the community when we compare our sample with samples in previous trials. ${ }^{15,42}$

Some limitations are also recognized. First, in our study, a placebo group was not included to control for contact time and attention due to lack of financial and human resources. However, given the seriousness of the problem, it is unlikely that concerns about falls were significantly reduced exclusively by social elements. ${ }^{14}$ Second, participants were not blinded; thus, they were aware of their group allocation, potentially introducing bias. Thirdly, our final follow-up assessment was conducted 12 months after baseline but only 7 months post intervention. This may hamper comparisons with other studies that applied a more common follow-up period of 12 months after the intervention. Our 7-month follow-up was guided by practical reasons (e.g., project finances) and comparability of effectiveness with AMB-NL. Lastly, dropout of study participants was substantial and different in both groups, i.e. $17 \%$ in the control group and $34 \%$ in the intervention group. Therefore, selective dropout may be an issue although additional analyses showed no significant differences on selected baseline characteristics including primary and secondary outcomes between those who completed the trial and those who did not in the two study groups.

Before the dissemination of AMB-Home on a larger scale, some changes may be considered given the experiences during this trial and a simultaneous process evaluation. ${ }^{41}$ First, the dropout rate in the intervention was considerable and a more effective and suitable procedure to screen older people is required for practice. A personal screening approach is recommended. During the nationwide implementation of the AMB-NL group program in the Netherlands this approach was applied and dropout rates reduced (from $42 \%$ dropout in the trial to $17 \%$ dropout in the implementation 
study). ${ }^{15,21}$ A face-to-face intake procedure (e.g., in general practitioner practices, falls clinics, or by nurses of home-care organizations) can simultaneously clarify the suitability and preference of potential participants of either program format, i.e. group-based or a home-based, for the older person. ${ }^{23}$ Second, improvements in compliance may add value to the program. The process evaluation ${ }^{41}$ indicated that the use of action plans decreased towards the end of the program; the use ranged from more than $70 \%$ in the first sessions to $51 \%$ in the latter sessions. Additionally, the 'exposure in vivo' exercise (i.e., performing an activity safely under supervision of the facilitator) was only performed by half of the participants. Difficulty in finding an appropriate activity was given as main reason by facilitators for not performing an activity together with the participant. It had been foreseen that selecting suitable activities for the goal-setting and action-planning components of the program could be challenging. ${ }^{43}$ To overcome this difficulty, 16 pictures of the Icon-FES ${ }^{44}$, which include the daily activities used in the assessment of concerns about falls by the FES-1 ${ }^{32}$, were incorporated in session 2 and served as examples for the selection of activities by the participant. Providing more attention to goal setting and the execution of personal action plans in the later sessions is needed to increase the program compliance and this will presumable achieve stronger program effects. Goal setting and behavioral practice are considered as the most promising behavioral change techniques in AMB-NL. ${ }^{45}$

Future studies may focus on defining the clinical relevance of the intervention effects, given that no criterion standards exist for levels of concerns about falls. Delbaere and colleagues (2010) suggested a cut-off point for the Falls Efficacy ScaleInternational; however, more research is needed on this relevant subject as meaningful cut-off scores may vary across different samples and settings. ${ }^{46}$ Also for the secondary outcomes no clinical relevant differences are known. In our study we have focused on meaningful changes according to $\mathrm{RCl}$-scores and effect sizes. The outcomes indicate small to medium program effects. Nevertheless, small statistical effects may have substantial impact on daily life. ${ }^{47}$ This is confirmed by the self-perceived benefits of the participants of the program. ${ }^{41}$ Another focus is the program's cost-effectiveness (in progress for ABM-Home) and the impact of individualizing the number of sessions, i.e., fitting the number of sessions to the anticipated effects regarding knowledge, skills and behavior. The latter would likely lead to a more cost-effective intervention. Future research may also focus on locating the most effective components within such complex interventions (e.g., education, action plans or 'exposure in vivo') and the effects of providing more attention to these components. ${ }^{45}$

\section{Conclusions}

In summary, AMB-Home reduced concerns about falls and associated avoidance of activity, as well as more downstream outcomes, such as disability and indoor falls in frail 
older people. The observed effects were small to medium, yet, present in a frail population and over a timeframe of 12-months. Therefore, this home-based, individualized $A M B$ format is a welcome addition to current geriatrics care, particularly for those persons who are not able or willing to attend group programs. Future geriatric research should focus on improving participants' and facilitators' compliance, and on determining the components that are essential to achieve an increase in program effects in this older population.

\section{Acknowledgments and funding}

We thank all participants and the nurses from the home-care organizations "Envida" (previously: "GroenekruisDomicura") in Maastricht, "Zorggroep Meander" in Heerlen and "Orbis Thuiszorg" in Sittard-Geleen for their participation. The Centre for Data and Information Management (MEMIC), Yvonne van Eijs, Inge van der Putten and Els Rennen are acknowledged for their assistance with the data collection. This research was funded by ZonMw, the Netherlands Organization for Health Research and Development (grant 120610001). The participation of author JV was supported by a grant from the Research Foundation, Flanders, Belgium (FWO Vlaanderen). 


\section{References}

1. Scheffer AC, Schuurmans MJ, van Dijk N, van der Hooft T, de Rooij SE: Fear of falling: measurement strategy, prevalence, risk factors and consequences among older persons. Age Ageing. 2008, 37(1):1924.

2. Zijlstra GA, van Haastregt JC, van Eijk JT, van Rossum E, Stalenhoef PA, Kempen GI: Prevalence and correlates of fear of falling, and associated avoidance of activity in the general population of communityliving older people. Age Ageing. 2007, 36(3):304-309.

3. Li F, Fisher KJ, Harmer P, McAuley E, Wilson NL: Fear of falling in elderly persons: association with falls, functional ability, and quality of life. J Gerontol B Psychol Sci Soc Sci. 2003, 58(5):P283-290.

4. Kressig RW, Wolf SL, Sattin RW, O'Grady M, Greenspan A, Curns A, Kutner M: Associations of demographic, functional, and behavioral characteristics with activity-related fear of falling among older adults transitioning to frailty. J Am Geriatr Soc. 2001, 49(11):1456-1462.

5. Deshpande N, Metter EJ, Lauretani F, Bandinelli S, Guralnik J, Ferrucci L: Activity restriction induced by fear of falling and objective and subjective measures of physical function: a prospective cohort study. $J$ Am Geriatr Soc. 2008, 56(4):615-620.

6. Cumming RG, Salkeld G, Thomas M, Szonyi G: Prospective study of the impact of fear of falling on activities of daily living, SF-36 scores, and nursing home admission. J Gerontol A Biol Sci Med Sci. 2000, 55(5):M299-305.

7. Boyd R, Stevens JA: Falls and fear of falling: burden, beliefs and behaviours. Age Ageing. 2009, 38(4):423-428.

8. Delbaere K, Close JC, Brodaty H, Sachdev P, Lord SR: Determinants of disparities between perceived and physiological risk of falling among elderly people: cohort study. BMJ. 2010, 341:c4165.

9. Denkinger MD, Lukas A, Nikolaus T, Hauer K: Factors associated with fear of falling and associated activity restriction in community-dwelling older adults: a systematic review. Am J Geriatr Psychiatry. 2015, 23(1):72-86.

10. Hadjistavropoulos T, Delbaere K, Fitzgerald TD: Reconceptualizing the role of fear of falling and balance confidence in fall risk. J Aging Health. 2011, 23(1):3-23.

11. Bula CJ, Monod S, Hoskovec C, Rochat S: Interventions aiming at balance confidence improvement in older adults: an updated review. Gerontology. 2011, 57(3):276-286.

12. Zijlstra GA, van Haastregt JC, van Rossum E, van Eijk JT, Yardley L, Kempen GI: Interventions to reduce fear of falling in community-living older people: a systematic review. J Am Geriatr Soc. 2007, 55(4):603615.

13. Kendrick D, Kumar A, Carpenter H, Zijlstra GAR, Skelton DA, Cook JR, Stevens Z, Belcher CM, Haworth D, Gawler SJ et al: Exercise for reducing fear of falling in older people living in the community. Cochrane $D b$ Syst Rev. 2014, 11.

14. Tennstedt S, Howland J, Lachman M, Peterson E, Kasten L, Jette A: A randomized, controlled trial of a group intervention to reduce fear of falling and associated activity restriction in older adults. J Gerontol B Psychol Sci Soc Sci. 1998, 53(6):P384-392.

15. Zijlstra GA, van Haastregt JC, Ambergen T, van Rossum E, van Eijk JT, Tennstedt SL, Kempen GI: Effects of a multicomponent cognitive behavioral group intervention on fear of falling and activity avoidance in community-dwelling older adults: results of a randomized controlled trial. J Am Geriatr Soc. 2009, 57(11):2020-2028.

16. Ullmann G, Williams HG, Plass CF: Dissemination of an evidence-based program to reduce fear of falling, South Carolina, 2006-2009. Prev Chronic Dis. 2012, 9:E103.

17. Smith ML, Jiang L, Ory MG: Falls efficacy among older adults enrolled in an evidence-based program to reduce fall-related risk: sustainability of individual benefits over time. Fam Community Health. 2012, 35(3):256-263.

18. Batra A, Melchior M, Seff L, Frederick N, Palmer RC: Evaluation of a community-based falls prevention program in South Florida, 2008-2009. Prev Chronic Dis. 2012, 9:E13. 
19. Ory MG, Smith ML, Wade A, Mounce C, Wilson A, Parrish R: Implementing and disseminating an evidence-based program to prevent falls in older adults, Texas, 2007-2009. Prev Chronic Dis. 2010, 7(6):A130.

20. Healy TC, Peng C, Haynes MS, McMahon EM, Botler JL, Gross L: The feasibility and effectiveness of translating a matter of balance into a volunteer lay leader model. J App/ Gerontol. 2008, 27(1):34-51.

21. Zijlstra GA, van Haastregt JC, Du Moulin MF, de Jonge MC, van der Poel A, Kempen Gl: Effects of the implementation of an evidence-based program to manage concerns about falls in older adults. Gerontologist. 2013, 53(5):839-849.

22. van Haastregt JC, Zijlstra GA, van Rossum E, van Eijk JT, de Witte LP, Kempen GI: Feasibility of a cognitive behavioural group intervention to reduce fear of falling and associated avoidance of activity in community-living older people: a process evaluation. BMC Health Serv Res. 2007, 7:156.

23. Dorresteijn TA, Rixt Zijlstra GA, Van Eijs YJ, Vlaeyen JW, Kempen GI: Older people's preferences regarding programme formats for managing concerns about falls. Age Ageing. 2012, 41(4):474-481.

24. Dorresteijn TA, Zijlstra GA, Delbaere K, van Rossum E, Vlaeyen JW, Kempen GI: Evaluating an in-home multicomponent cognitive behavioural programme to manage concerns about falls and associated activity avoidance in frail community-dwelling older people: Design of a randomised control trial [NCT01358032]. BMC Health Serv Res. 2011, 11:228.

25. Gezondheidsraad: Preventie bij ouderen: focus op zelfredzaamheid. Den Haag: Gezondheidsraad; 2009.

26. UK Government: Government response to the house of lords select committee on public service and demographic change report of session 2012-13: 'ready for ageing?'. London: www.gov.uk; 2013.

27. Community Living Council: U.S. Department of health and human services strategic plan for community living, FY 2014-FY 2018. Washington, DC: Department of health and human services; 2014.

28. Swain DG, Nightingale PG: Evaluation of a shortened version of the Abbreviated Mental Test in a series of elderly patients. Clin Rehabil. 1997, 11(3):243-248.

29. Kempen GI, Meier AJ, Bouwens SF, van Deursen J, Verhey FR: [The psychometric properties of the Dutch version of the telephone interview cognitive Status (TICS)]. Tijdschrift voor Gerontologie en Geriatrie. 2007, 38(1):38-45.

30. Vlaeyen JWS, de Jong J, Geilen M, Heuts PHTG, van Breukelen G: The treatment of fear of movement/(re)injury in chronic low back pain: further evidence on the effectiveness of exposure in vivo. Clin J Pain. 2002, 18(4):251-261.

31. Resnicow K, Dilorio C, Soet JE, Borrelli B, Hecht J, Ernst D: Motivational interviewing in health promotion: It sounds like something is changing. Health Psychol. 2002, 21(5):444-451.

32. Yardley L, Beyer N, Hauer K, Kempen G, Piot-Ziegler C, Todd C: Development and initial validation of the falls efficacy scale-international (FES-I). Age Ageing. 2005, 34(6):614-619.

33. Kempen GI, Todd CJ, Van Haastregt JC, Zijlstra GA, Beyer N, Freiberger E, Hauer KA, Piot-Ziegler C, Yardley L: Cross-cultural validation of the falls efficacy scale international (FES-I) in older people: results from Germany, the Netherlands and the UK were satisfactory. Disabil Rehabil. 2007, 29(2):155-162.

34. Zijlstra G, Dorresteijn T, Vlaeyen J, Kempen GI: Measuring Avoidance Behavior Due to Fear of Falling in Community-Living Older Adults. Gerontologist. 2013, 53:199-199.

35. Kempen GI, Miedema I, Ormel J, Molenaar W: The assessment of disability with the Groningen Activity Restriction Scale. Conceptual framework and psychometric properties. Soc Sci Med. 1996, 43(11):16011610.

36. Lamb SE, Jorstad-Stein EC, Hauer K, Becker C, Prevention of Falls Network E, Outcomes Consensus G: Development of a common outcome data set for fall injury prevention trials: the Prevention of Falls Network Europe consensus. J Am Geriatr Soc. 2005, 53(9):1618-1622.

37. CBS: Standaard Onderwijsindeling 2006. Editie 2009/'10. Voorburg/Heerlen: Centraal Bureau voor de Statistiek, The Netherlands; 2009.

38. Cohen J: A power primer. Psychol Bull. 1992, 112(1):155-159.

39. Evans C, Margison F, Barkham M: The contribution of reliable and clinically significant change methods to evidence-based mental health. Evidence Based Mental Health. 1998, 1(3):70-72. 
40. Jacobson NS, Truax P: Clinical significance: a statistical approach to defining meaningful change in psychotherapy research. J Consult Clin Psychol. 1991, 59(1):12-19.

41. Dorresteijn TA, Rixt Zijlstra GA, Van Haastregt JC, Vlaeyen JW, Kempen GI: Feasibility of a nurse-led inhome cognitive behavioral program to manage concerns about falls in frail older people: a process evaluation. Res Nurs Health. 2013, 36(3):257-270.

42. Metzelthin SF, van Rossum E, de Witte LP, Ambergen AW, Hobma SO, Sipers W, Kempen GI: Effectiveness of interdisciplinary primary care approach to reduce disability in community dwelling frail older people: cluster randomised controlled trial. BMJ. 2013, 347:f5264.

43. Daniels R: Frail elderly identification and disability prevention in primary care [PhD thesis]. Maastricht: Faculty of Health, Medicine and Life Sciences, University Maastricht; 2011.

44. Delbaere K, S TS, Lord SR: Development and initial validation of the iconographical falls efficacy scale. J Gerontol a-Biol. 2011, 66(6):674-680.

45. Vestjens L, Kempen GI, Crutzen R, Kok G, Zijlstra GA: Promising behavior change techniques in a multicomponent intervention to reduce concerns about falls in old age: a Delphi study. Health Educ Res. 2015, 30(2):309-322.

46. Delbaere K, Close JC, Mikolaizak AS, Sachdev PS, Brodaty H, Lord SR: The falls efficacy scale international (FES-I). A comprehensive longitudinal validation study. Age Ageing. 2010, 39(2):210-216.

47. Lipsey MW, Wilson DB: The efficacy of psychological, educational, and behavioral treatment. Confirmation from meta-analysis. Am Psychol. 1993, 48(12):1181-1209. 

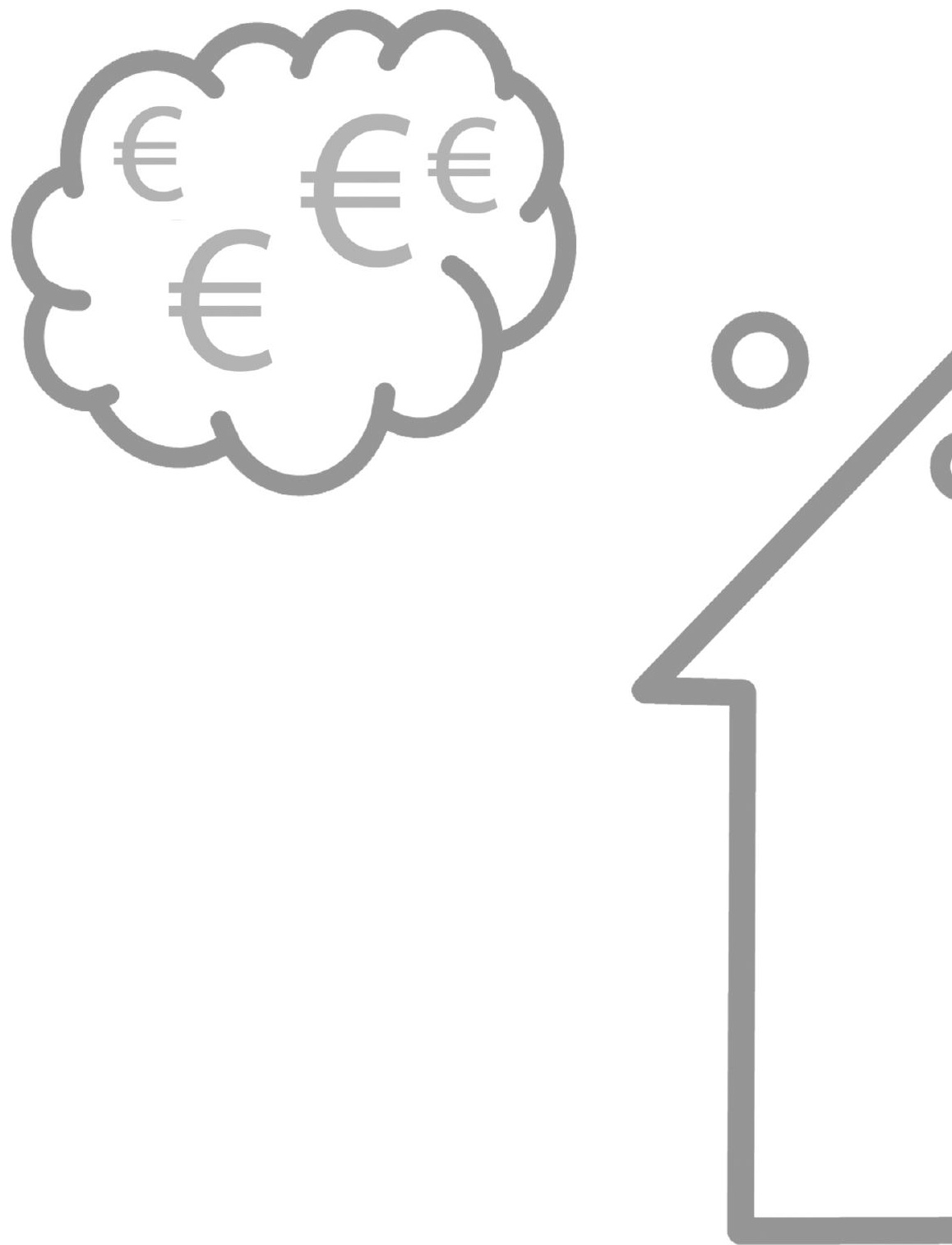


\section{Chapter 6}

Cost-effectiveness of a home-based program to reduce concerns about falls in frail, community-dwelling older people: evidence from a randomized controlled trial 


\begin{abstract}
Background Concerns about falls, also often labelled as fear of falling, are frequently reported by older people. These concerns can have serious consequences such as an increase of falls. The aim of this study was to evaluate the cost-effectiveness of a homebased, cognitive behavioral program for community-dwelling, frail older people compared to usual care from a societal perspective.
\end{abstract}

Methods This economic evaluation was embedded in a randomized controlled trial with a follow-up time of 12-months. In the trial 389 people aged 70 years or older in the Netherlands were allocated to a usual care $(n=195)$ or intervention group $(n=194)$. The usual care group received care according to the Dutch standards. The intervention group received, besides usual care, a home-based, cognitive behavioral program. The program consisted of seven sessions aimed to instill adaptive and realistic views about fall risks via cognitive restructuring and to increase activity and safe behavior using goal setting and action planning. The main outcome measure for the cost-effectiveness analysis (CEA) was concerns about falls and the outcome for the cost-utility analysis (CUA) was Quality Adjusted Life Years (QALYs). Furthermore, several sensitivity analyses (e.g., per-protocol and healthcare perspective analysis) were performed.

Results The total societal costs for the usual care group were 8,094 Euro and 7,890 Euro for participants in the intervention group of which the program costs were 716 Euro per participant. After synthesizing the effects and costs, the probability that the intervention is cost-effective for concerns about falls is $100 \%$ with a willingness to pay of 1,300 Euro, and the probability that the intervention is cost-effective is $75 \%$ at a willingness to pay of 20,000 Euro per QALY. Sensitivity analyses showed that if participants received at least five of the seven sessions the probability that the program was cost-effective increased, while as only the costs from a healthcare perspective were taken the probability of a cost-effective program decreased.

Conclusions The program is likely to be cost-effective, and therefore this home-based program is a useful addition to current geriatric care, particularly for those persons who are not able or willing to attend group programs.

Trial registration NCT01358032 


\section{Background}

Concerns about falls, also often labelled as fear of falling, are a common health problem and a threat to autonomy for community-dwelling older people. Dependent on the target sample and way of measurement, prevalence rates from $20 \%$ to $85 \%$ are reported for independently living older adults. ${ }^{1}$ Concerns about falls are not only reported by those who have recently fallen, but also by those who have not. ${ }^{2,3}$ These concerns are associated with adverse outcomes in psychosocial, physical and functional domains such as loss of balance confidence, social isolation, symptoms of anxiety and depression, avoidance of daily activities, physical frailty, falls, loss of independence, and institutionalisation. ${ }^{1,4-10}$ In addition to the negative consequences of concerns about falls, there is the risk of increased healthcare usage and societal costs. ${ }^{7}$ Although, concerns about falls can be seen as a health problem apart from falls ${ }^{11}$, little is known about the societal costs.

Several reviews showed that different approaches may be successful in reducing concerns about falls ${ }^{12-14}$ and can potentially contribute to a better quality of life and independent living of older people. The multicomponent, cognitive behavioral program 'A Matter of Balance' (AMB) is one of the few interventions that targets psychosocial, physical as well as functional aspects of concerns about falls. ${ }^{15,16}$ This community-based group program proved to be successful in reducing concerns about falls and associated activity avoidance without an increase of falls. ${ }^{17,18}$ The program consistently shows effects on a broad range of outcomes and is considered preferable to usual care in terms of costs and effects in community-dwelling older people ${ }^{17-27}$ The program comprises eight group sessions and is led by trained healthcare professionals or volunteers and has been successfully implemented in different settings, i.e. in several states of the US and nationwide in the Netherlands. ${ }^{28-31}$ Despite its success, a substantial part of the (potential) participants do not attend or finish the program because of health problems. ${ }^{17,32}$ To enable frail, community-dwelling people to participate and to be consistent with their preferences ${ }^{33}$, we developed a tailor-made, home-based format (AMB-Home). ${ }^{34}$ This new program consists of seven individual sessions (including three home-visits and four telephone contacts). The principles of the AMB program were maintained in AMB-Home, namely to instill adaptive and realistic views about fall risks via cognitive restructuring and to increase activity and safe behavior using goal setting and action planning. Evaluation of the clinical effects of AMB-Home showed a significant decrease of concerns about falls in the intervention group compared to the usual care group. ${ }^{35}$ AMB-Home also demonstrated favorable effects regarding the reduction of associated avoidance of activity, disability and the number of indoor falls in the intervention group compared with the usual care group. ${ }^{35}$

Interventions that support active ageing as long as possible are important in ageing societies, also in view of managing healthcare costs. ${ }^{36}$ An economic evaluation of the group program of $\mathrm{AMB}$ in the Netherlands showed comparable costs in the control and 
intervention group with significant clinical effects and could therefore be considered as preferable to usual care. ${ }^{23}$ However, the impact of the AMB-Home program on healthrelated quality of life and costs, is not known yet. In this paper we report therefore the results of an economic evaluation using data obtained during the AMB-Home trial. The study aims to assess whether our individual, cognitive behavioral approach is preferable to care as usual in terms of costs and effects. We expected that AMB-Home leads to lower levels of concerns about falls and a higher quality of life without an increase in costs and is considered therefore cost-effective. The cost-effectiveness analysis is performed from a societal perspective (healthcare, as well patient and family costs).

\section{Methods}

\section{Study design and participants}

This economic evaluation was part of a two-group randomized controlled trial with a follow-up period of 12 -months. ${ }^{34}$ In this economic evaluation we performed a costeffectiveness analysis (CEA) and cost-utility analysis (CUA) by comparing the societal costs and effects of the intervention group with those of the usual care group from a societal perspective according to the Dutch guidelines. ${ }^{37}$ The reporting follows the Consolidated Health Economic Evaluation Reporting Standards (CHEERS) guidelines. ${ }^{38}$ The design of this study was described in detail elsewhere. ${ }^{34}$

The selection of participants was done in four consecutive cycles between March and December 2009 in three communities in the south of the Netherlands. The municipal registry offices of these communities randomly selected a total of 11,490 addresses of community-dwelling people of 70 years or over. To screen for eligibility, these people received a short postal questionnaire with a free return envelope, as well as information about the trial and an informed consent form. People were included when they reported at least sometimes concerns about falls as well as associated activity avoidance, and valued their perceived general health as fair or poor as assessed with one item of the MOS- $20^{39}$, and signed the informed consent. People were excluded if they were confined to bed, restricted by permanent wheelchair use, waiting for nursing home admission, or experienced substantial hearing, vision or cognitive impairments. Additionally, a restriction was applied to couples. Only one person of a couple was allowed to participate in the trial to prevent reciprocal influencing; lots were drawn to determine who to include. Participants were randomly allocated to either the usual care or the intervention group. A computerized two block stratified randomization was performed with one prognostic factor: the level of concerns about falls (sometimes, regular, often, and very often). The randomization took place directly after the baseline measurement and was conducted by an external agency blinded to participant characteristics. The intervention group was offered the seven sessions of AMB-Home to reduce concerns about falls, while the usual care group only received care according to the Dutch standards. The 
Medical Ethics Committee of the Maastricht University/Academic Hospital Maastricht in the Netherlands approved the study (MEC 07-3-064).

\section{Intervention and usual care group}

'A Matter of Balance at Home' (AMB-Home) is an individual, cognitive behavioral program to manage concerns about falls. The program comprises seven individual sessions, consisting of three home-visits of 60,60 and 75 minutes, respectively, and four telephone contacts of 35 minutes each. As mentioned, it is founded on the evidence based group program A Matter of Balance (AMB) that uses principles of cognitive restructuring, social modelling, education and other strategies for behavioral change. ${ }^{17,18,40}$ The purpose of the program is shifting from maladaptive to adaptive cognitions with respect to falling and concerns about falls, instilling a realistic view of fall risk, increasing selfefficacy beliefs and feelings of control, and changing behavior of the participant. To obtain these goals the following strategies are applied: 1 ) identifying and restructuring misconceptions about falls and fall risk with e.g. discussions and checklists; 2) setting realistic personal goals for increasing activity levels and safe behavior with e.g. action plans; and 3) promoting the uptake of old and new daily life activities that have been avoided owing to concerns about falls with e.g. exposure in vivo. The program was conducted by community nurses $(n=8)$ who were qualified in the field of geriatrics and worked at local home-care agencies. More details about the program are published elsewhere. ${ }^{34,41}$

Since no standard treatment for concerns about falls was available during the study period it is likely that participants in the usual care group received no treatment. Like the participants in the intervention group, they may have received usual care from health and community service providers or informal caregivers, depending on their needs.

\section{Clinical outcomes}

The clinical outcome for the CEA was concerns about falls assessed by the 16-item Falls Efficacy Scale-International (FES-I). Participants were asked to indicate how concerned they are about falling while carrying out several activities of daily living ( 1 = not all concerned to 4 = very concerned; range of the scale 16-64). ${ }^{42,43}$ Avoidance behavior due to concerns about falls was measured with the 16-item Falls Efficacy Scale-International Avoidance Behavior (FES-IAB). ${ }^{35,44}$

The Health Survey Short Form (SF-12) $*^{1}$ was used to measure both health-related quality of life ${ }^{45}$ and utilities for the cost-utility analysis (CUA). The SF-12 is a selfadministered questionnaire, which is widely used as quality of life and utility instrument. The SF-12 (called SF-6D when it is used for economic evaluations) results in

\footnotetext{
${ }^{1}$ For the SF12v1, Standard Recall, Netherlands (Dutch) an unlicensed version was used that deviates from the official version on the item about pain. Pain perception is asked instead of pain as barrier. As such, the results should be interpreted with care when compared to SF12v1 scores from other studies (48)
} 
health states. Utility values are calculated for these health states, using preferences elicited from a general population, the so-called Brazier algorithm. ${ }^{46}$ The value of the utility theoretically ranges from 1 , representing the best possible health state, to 0 , equal to death, to below zero, states worse than death. ${ }^{47}$ These utilities were used to calculate Quality Adjusted Life Time Years (QALYs). This was done by multiplying the disease burden survival (quantity of life lived) by utilities (quality of life lived). ${ }^{47}$

\section{Health service utilization and costs}

The economic evaluation is carried out from a societal perspective. This means that all costs are taken into account, irrespective of who bears the costs or gains the benefits. In this study we have assessed (1) intervention costs, (2) healthcare costs, and (3) patient and family costs. First, the costs of the program consisted of used materials, salaries of the facilitators, costs of training sessions for the facilitators etc. Second, the healthcare costs that were included were hospital visits (inpatient and outpatient treatment), GP consultations, and visits to paramedics. Third, the patient and family costs included (nursing) home-care, informal care, aids, and in-home modifications. Because of the complex relation between concerns about falls and a wide range of outcomes in the domains of physical, mental and social health, no distinction could be made between costs related to concerns about falls and other health care costs. Loss of productivity was not included, because all persons were above the age of retirement in the Netherlands.

Cost data were collected by means of a (monthly) cost dairy for one year. ${ }^{49}$ Participants were asked to report their use of healthcare services and the patient and family costs on a form and had to send this in each month. Participants who did not return the form or had missing data were contacted by phone to ensure completion of data.

In order to estimate the costs, the volumes were multiplied by the assigned cost price of that unit. Cost prices were obtained from the Dutch guidelines for cost analysis in health care research. ${ }^{37}$ If these costs were not available in the guideline estimation of the costs by using retail prices of professional organizations. Costs of healthcare devices, aids and in-home adaptations were estimated by looking on the internet for retail prices from suppliers in the Netherlands. For each product, the average price was used. All costs are expressed in 2011 Euros $(€)$ and if necessary indexed to the baseline year using a consumer price index as suggested in the manual. ${ }^{37}$ An overview of all used prices can be found in Additional file 1. Since the time frame in which costs and effects occurred was relatively short (one year for the individual participant), discounting (a correction for time preference) was not necessary. ${ }^{47}$

\section{Synthesizing cost and effects}

To examine cost-effectiveness and cost-utility, incremental cost-effectiveness ratios (ICERs) and incremental cost-utility ratios (ICURs) were calculated. ICERs and ICURs were calculated by dividing the difference in costs by the difference in effects (in con- 
cerns about falls and in QALYS) between the usual care group and the intervention group. The ICERs and ICURs were considered as the incremental cost per unit of additional effect. ${ }^{47}$

The ICERs were plotted in a cost-effectiveness plane (CE-plane), in which the x-axis shows the difference in effect between the intervention and usual care, and the $y$-axis the differences in costs between the intervention and usual care. In a CE-plane, four quadrants are shown; ICERs located in the North East (NE) indicate that the intervention was more effective and more costly as compared with usual care. ICERs in the South East (SE), the dominant quadrant, indicate that the intervention is more effective and less costly. ICERs in the South West (SW) indicate that the intervention was less effective and less costly, and ICERs located in the North West (NW), the inferior quadrant, indicate that the intervention was less effective and more costly. ${ }^{47}$

An intervention is preferred above usual care when there are higher effects with lower costs and not preferred when the intervention has lower effects against higher costs compared to usual care. Yet, in case of higher effects and higher costs, or lower effects and lower costs, the preference for an intervention depends on how much society is willing to pay for a certain gain in effect. In the Netherlands the willingness to pay (WTP) to gain one QALY ranges from 20,000 Euro to 80,000, depending on the disease burden. ${ }^{50}$ In a recent rapport of the Dutch health institute the disease burden in the Netherlands is classified into three categories, namely low with WTP till €20,000/QALY; middle with WTP till €50,000/QALY; and high with WTP till €80,000/QALY. ${ }^{51}$ The disease burden of concerns about falls is not known, however, many older people see the risk of falling and concerns about falls as part of the aging process ${ }^{52}$, therefore we used a WTP of $€ 20,000 /$ QALY, which is also often used for preventive interventions. ${ }^{50,51}$ Like for almost all clinical outcomes, no acceptable WTP level has been defined for concerns about falls so far, therefore only a range is described showing the likelihood that the intervention is cost-effective at different thresholds, i.e. Euro values for 1 point improvement in concerns about falling.

\section{Sensitivity analyses}

As the results of an economic evaluation are always influenced by uncertainty it is common to perform a number of sensitivity analyses to show the results of analyses with alternative assumptions. We performed sensitivity analyses for per-protocol analyses for the reason that attending five sessions was considered to be minimally sufficient for intervention exposure. ${ }^{17,18}$ Furthermore, sensitivity analyses were performed from a healthcare perspective (only medical costs) to allow comparison of the outcomes with other studies, since many studies exclusively applied this perspective. Finally, two additional sensitivity analyses were performed, i.e. an analysis without extreme cost outliers and an analysis with avoidance of activity due to concerns about falls as outcome to study the cost-effectiveness of the behavioral component of the 
program. Outliers were defined by a boxplot in which a point beyond the upper outer fence was considered an extreme outlier. ${ }^{53}$

\section{Statistical analysis}

The intention-to-treat principle was used for the primary (base-case) analyses, including all participants with valid data on costs (at least $75 \%$, i.e. 9 out of 12 months) and clinical outcomes at 12-months, regardless of whether they received the (complete) intervention or not.

Missing values for the FES-I were imputed at the level of the scale by means of multiple imputations. ${ }^{54}$ Missing values for the SF-12 were replaced by the mean of the group. ${ }^{46}$ The maximum number of missing values for both outcomes was set on $25 \%$. Missing values for the cost data were imputed by linear interpolation (i.e., imputation with participants' mean score on the previous and next measurement).

Clinical outcomes were assessed using mixed-effects linear regression analyses. Models were adjusted for the baseline value of the outcome measure, age, gender, perceived general health, and number of falls in the 6 months before baseline and concerns about falls. These covariates were considered a priori as relevant to the outcomes. ${ }^{34}$ The level of statistical significance was set at .05 , one-tailed, because we expected an improvement on the outcomes.

Bootstrapping $(1,000 \times)$ was used to estimate the sampling distribution and to construct confidence intervals around mean costs. In this nonparametric method an estimation of the sampling distribution was made by simulation of the original data many times. Bootstrapping is needed because of the presence of relatively few patients with high costs and the absence of negative costs, like is typical in economic evaluations. Furthermore, bootstrapping $(5,000 \times)$ was used to calculate the uncertainty around the ICERs. Before cost-effectiveness planes (CE-planes) and incremental cost-effectiveness acceptability curves (ICEA-curve) were plotted, the total scores on the FES-I and FES-IAB were mirrored; in that way a high score on the FES-I or FES-IAB means a better state, like already holds for QALYs as outcome. ${ }^{55,56}$

Statistical analyses were performed in SPSS 21.0.1 (SPSS, Inc., Chicago, IL); bootstrapping was done in MS Excel 2010.

\section{Results}

The flow of participants during the trial is presented in Figure 1. Eligibility screening occurred in the general community (see Study Design). Through randomization, 195 participants were allocated to the usual care group, and 194 participants were included 


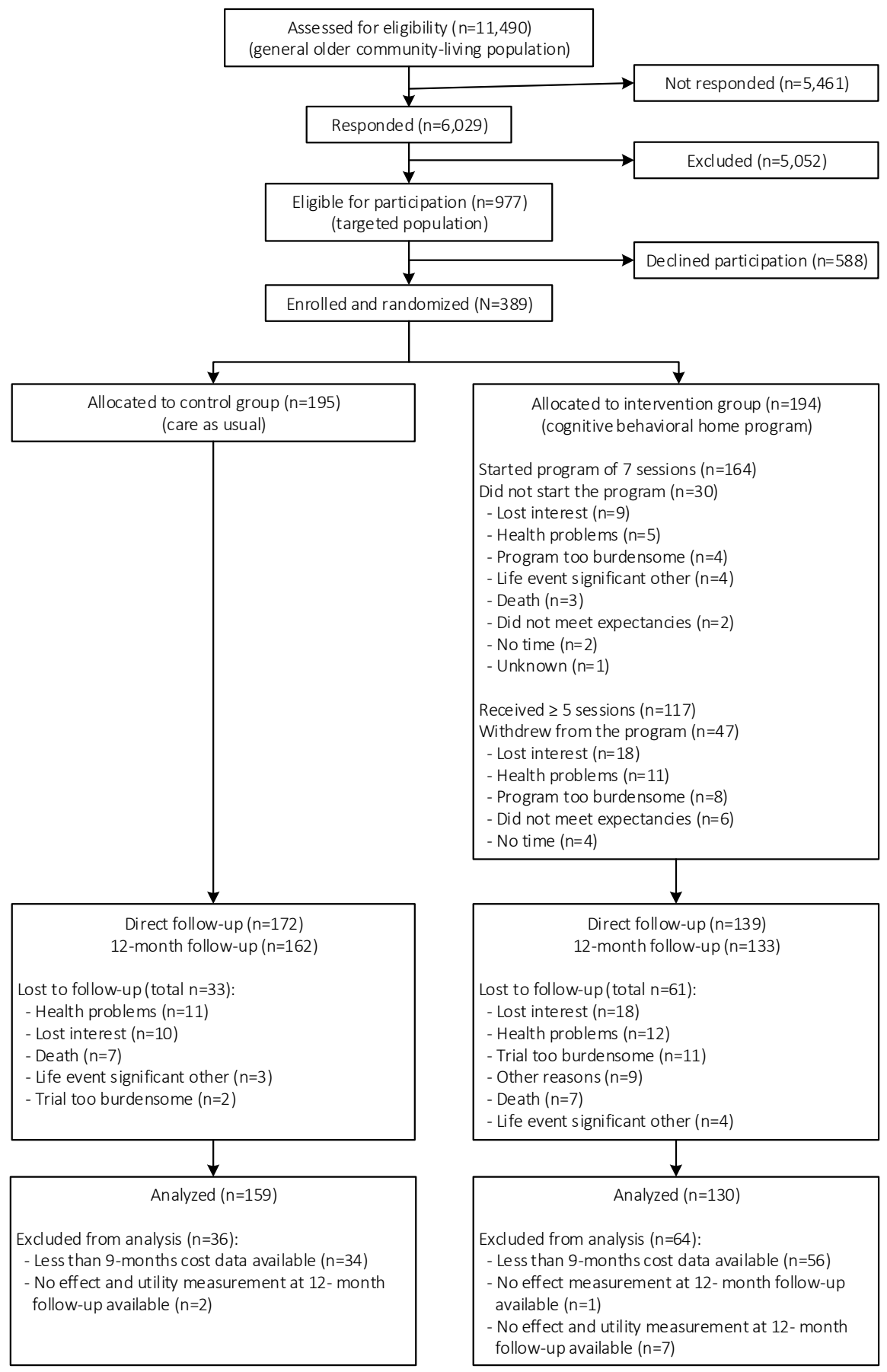

Figure 1 Flow of participants through the trial 
in the intervention group. The program was started by 164 participants (85\%) out of the 194 participants, and 117 participants (60\%) received at least five of the seven program sessions. In the usual care group 162 participants completed the trial and 159 (82\%) of them had complete data, whereas 133 participants in the intervention group completed the trial and 130 (67\%) of them had complete data. Withdrawal was highest at the 5month follow-up measurement, which was directly after the intervention period. The main reasons for lost to follow-up were similar in the usual care and intervention group, i.e., lost interest and health problems. Baseline characteristics were comparable in both groups (Table 1). No significant differences were identified regarding baseline characteristics between dropouts in the intervention and usual care group (not tabulated).

Table 1 Baseline characteristics and outcomes $(n=289)$

\begin{tabular}{|c|c|c|c|c|}
\hline & \multicolumn{2}{|c|}{$\begin{array}{l}\text { Usual care group } \\
\mathrm{n}=159\end{array}$} & \multicolumn{2}{|c|}{$\begin{array}{l}\text { Intervention group } \\
\mathrm{n}=130\end{array}$} \\
\hline \multicolumn{5}{|l|}{ Demographic } \\
\hline Female (\%) & 117 & $(73.6)$ & 84 & $(64.6)$ \\
\hline Mean age in years (SD) & 77.7 & $(5.2)$ & 78.0 & $(5.4)$ \\
\hline Living alone (\%) & 96 & $(60.4)$ & 74 & $(56.9)$ \\
\hline Low education level (\%) & 77 & $(48.4)$ & 72 & $(55.4)$ \\
\hline \multicolumn{5}{|l|}{ Health-related } \\
\hline Poor perceived general health (\%) & 14 & $(8.8)$ & 15 & $(11.5)$ \\
\hline Mean number of active chronic diseases (SD) & 1.6 & $(0.9)$ & 1.7 & $(0.9)$ \\
\hline SF-6D classification index $(<0-\underline{1})(S D)$ & 0.65 & $(0.1)$ & 0.65 & $(0.1)$ \\
\hline \multicolumn{5}{|l|}{ Fall-related } \\
\hline Once or more falls in the past 6 months (\%) & 87 & $(54.7)$ & 83 & $(63.9)$ \\
\hline Regular till very often concerned to fall (\%) & 81 & $(50.9)$ & 68 & $(52.3)$ \\
\hline Regular till very often avoid activities (\%) & 73 & $(45.9)$ & 69 & $(53.1)$ \\
\hline FES-I (16-64) (SD) & 35.1 & $(9.3)$ & 35.1 & $(9.9)$ \\
\hline FES-IAB (16-64) (SD) & 28.6 & $(9.1)$ & 28.0 & (9.0) \\
\hline
\end{tabular}

*Underlined scores=most favorable scores.

\section{Costs}

As shown in Table 2, the intervention mean costs were 716 Euro per participant in the intervention group. Total mean costs in the usual care group were 8,094 Euro and 7,890 Euro (including intervention costs) in the intervention group. None of the (sub)categories, e.g. healthcare or family and patient costs, showed a significant difference in costs between both groups. The volumes per category are tabulated in Additional file 2 . 


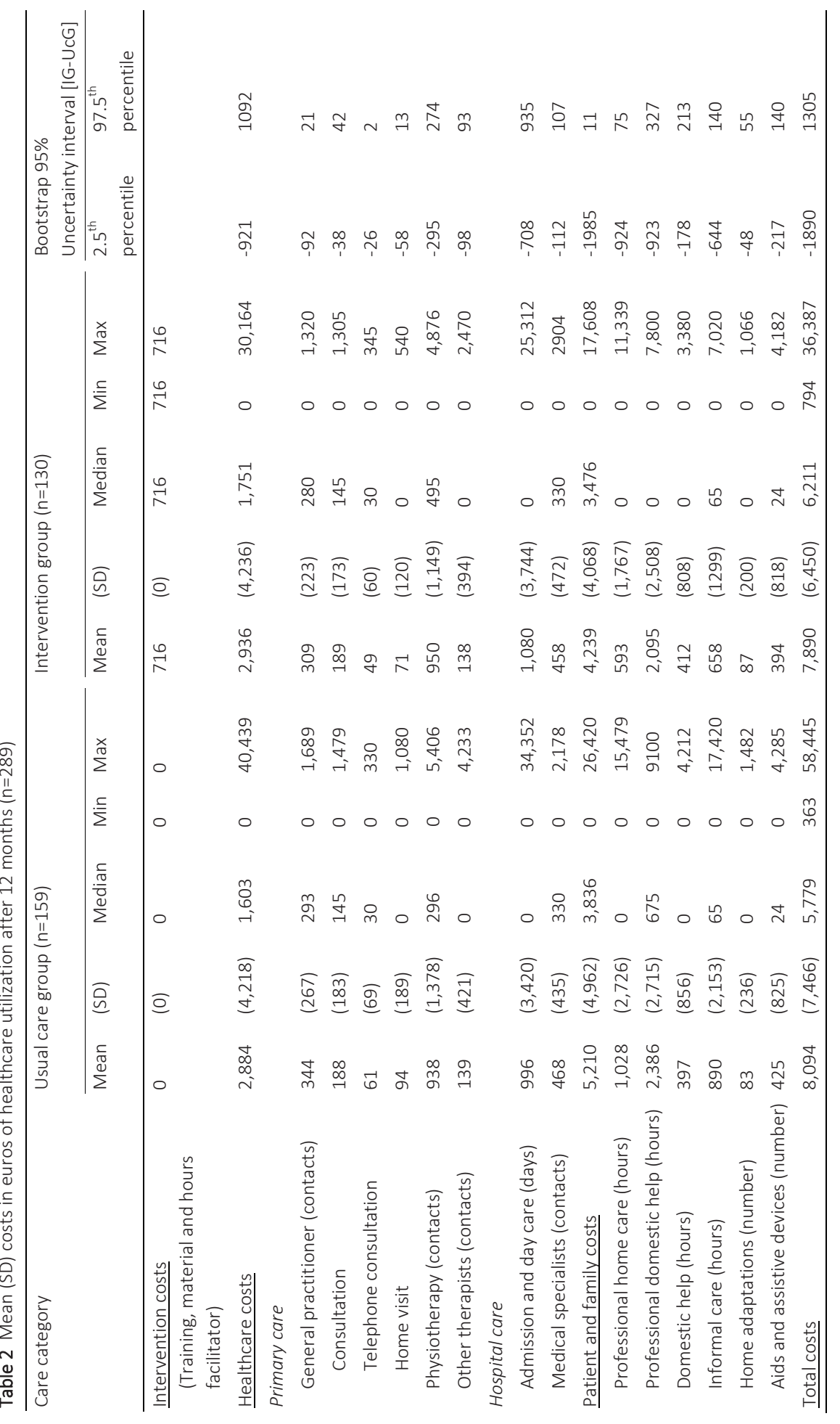




\section{Clinical outcomes}

The intervention group showed a significant decrease in concerns about falls compared with the usual care group. The estimated program effect from baseline to 12 months follow-up was 3.92 points on the FES-I (adjusted mean difference) $(95 \% \mathrm{Cl}:-\infty--2.52 ; P$ $<0.001) .{ }^{35}$ At 12 months follow-up no significant difference in quality of life was found between the intervention and usual care group (0.016 point on SF-6D (adjusted mean difference) $95 \% \mathrm{Cl}:-0.003-0.036 ; P=0.16)$.

\section{Cost-effectiveness}

Comparing the costs and effects of the AMB-Home program to the usual care group, the intervention was preferred over usual care (see Table 3). The ICEA-curve (Figure 2) indicates that the probability of AMB-Home being cost-effective was 60 to $100 \%$ with a range from 0 to 1500 Euro for the WTP thresholds. Also can be seen that the probability of AMB-Home being cost-effective was already $100 \%$ at a WTP of 1,300 Euro for an improvement for concerns about falls. This implies that when the society is willing to pay 1,300 Euro, the program is certainly cost-effectiveness for one point increase on the FES-I. The cost-effectiveness of the program for a WTP of 20,000 Euro for one QALY was $75 \%$. This implies that when the society is willing to pay 20,000 , there is a $75 \%$ probability that program is cost-effectiveness for one more year in optimal health.

Table 3 Cost-effectiveness analyses and sensitivity analyses for concerns about falls and QALY

\begin{tabular}{|c|c|c|c|c|c|c|c|}
\hline & \multirow[b]{2}{*}{$\begin{array}{l}\text { Usual care } \\
\text { group }\end{array}$} & \multirow{2}{*}{\multicolumn{2}{|c|}{$\begin{array}{l}\text { Intervention ICER } \\
\text { group }\end{array}$}} & \multicolumn{4}{|c|}{ Distribution of cost-effectiveness plane } \\
\hline & & & & $N E^{b} \%$ & $\begin{array}{l}\mathrm{NW}^{\mathrm{C}} \% \\
\text { (inferior) }\end{array}$ & $S W^{d} \%$ & $\begin{array}{l}\mathrm{SE}^{\mathrm{e}} \% \\
\text { (dominant) }\end{array}$ \\
\hline \multicolumn{8}{|l|}{ Concerned about falls ${ }^{f}$} \\
\hline $\begin{array}{l}\text { Concerned about falls } \\
\text { base case }\end{array}$ & 159 & 130 & -49 & 40 & 0 & 0 & 60 \\
\hline Per-protocol & 159 & 104 & -178 & 20 & 0 & 0 & 79 \\
\hline Healthcare perspective & 159 & 130 & 13 & 54 & 0 & 0 & 46 \\
\hline Without outliers & 158 & 128 & -77 & 33 & 0 & 0 & 67 \\
\hline Activity avoidance ${ }^{h}$ & 159 & 130 & -66 & 39 & 1 & 0 & 60 \\
\hline \multicolumn{8}{|l|}{$Q A L Y^{g}$} \\
\hline QALY base case & 159 & 130 & -9.649 & 38 & 3 & 1 & 57 \\
\hline Per-protocol & 159 & 104 & -39.270 & 17 & 4 & 6 & 74 \\
\hline Healthcare perspective & 159 & 130 & 2.446 & 51 & 4 & 1 & 44 \\
\hline Without outliers & 158 & 128 & -15.188 & 30 & 3 & 2 & 65 \\
\hline
\end{tabular}

${ }^{a}$ Incremental cost-effectiveness ratio

${ }^{b}$ North East quadrant: the intervention was more effective and more costly as compared to usual care 'South East quadrant: the intervention was more effective and less costly as compared to usual care

${ }^{d}$ North West quadrant: the intervention was less effective and more costly as compared to usual care

'South West quadrant: the intervention was less effective and less costly as compared to usual care

${ }^{f}$ Measured with the Falls Efficacy Scale-International

${ }^{g}$ Quality adjusted life years measured with the SF-6D

${ }^{\mathrm{h}}$ Measured with the Falls Efficacy Scale-Avoidance Behavior International 
Base case
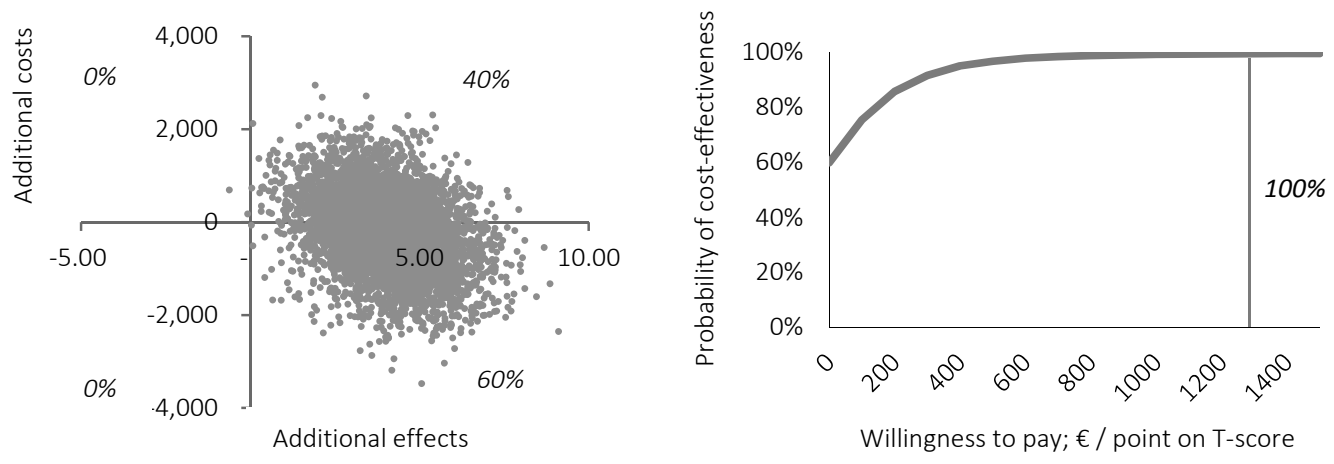

Per-protocol
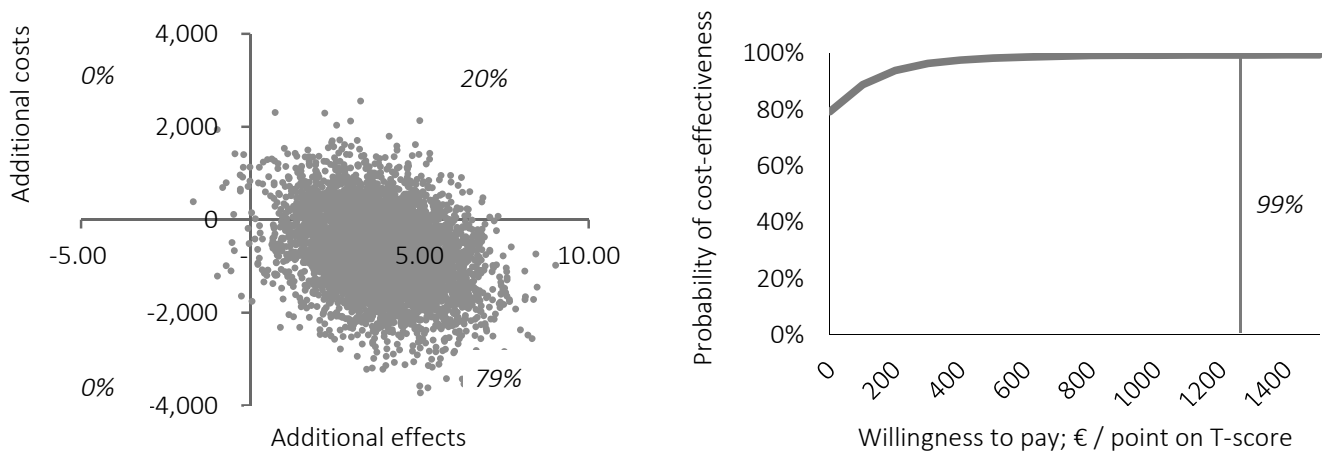

Healthcare perspective
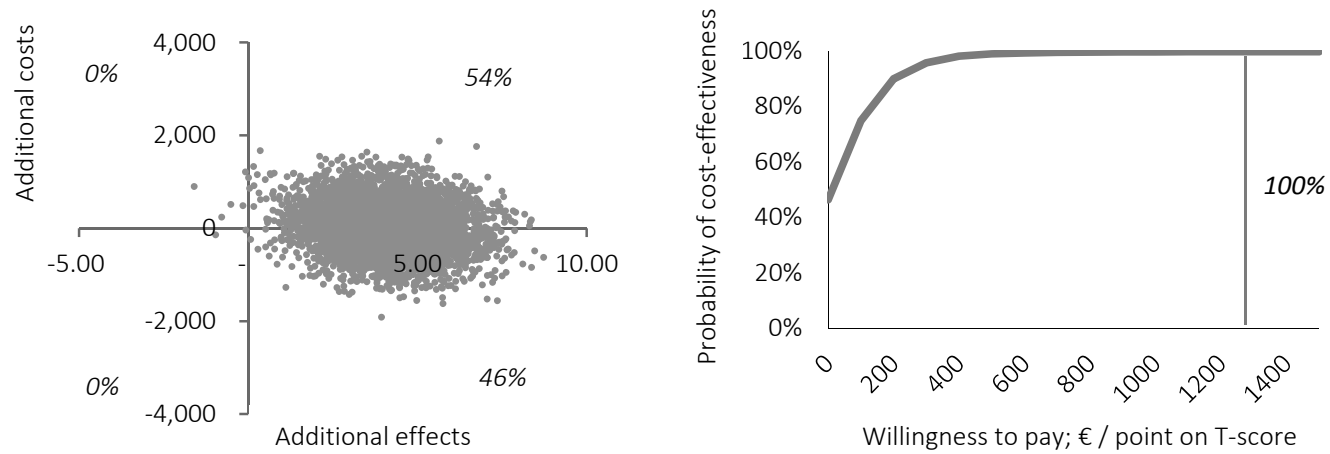
Without outliers
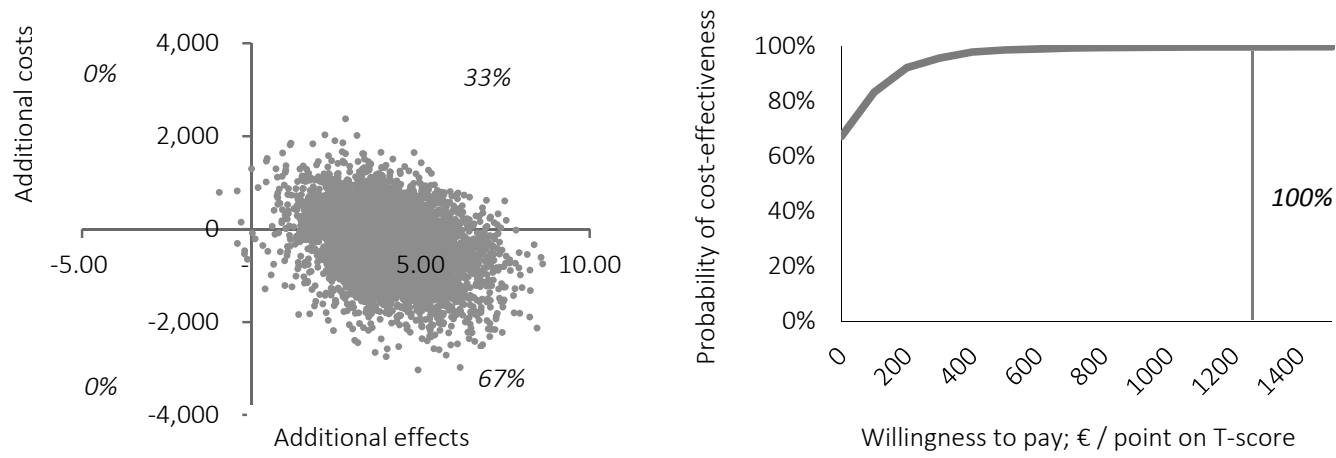

Activity avoidance
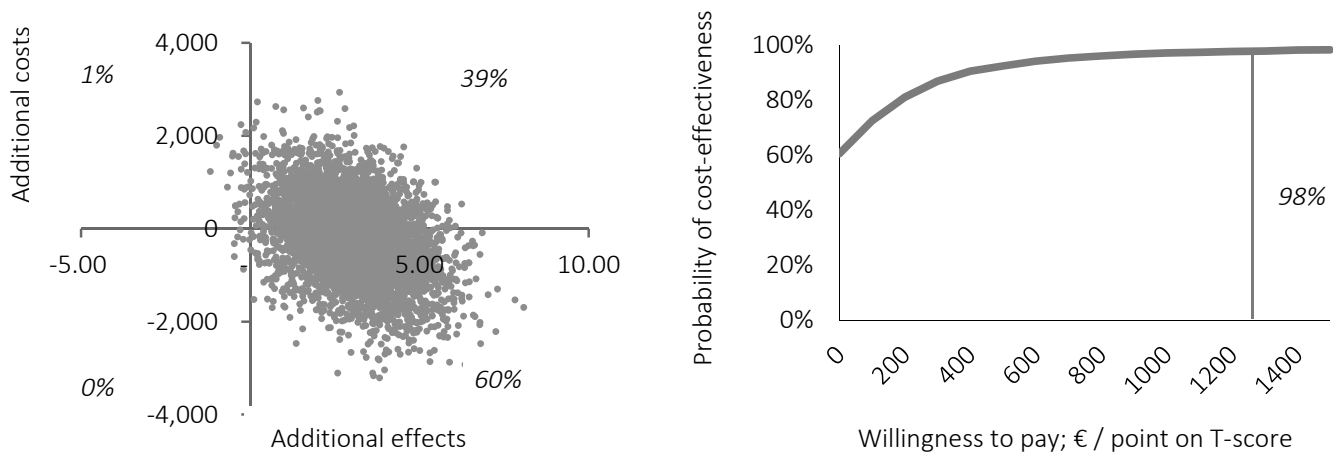

Figure 2 Cost-effectiveness planes (CE-planes) and incremental cost-effectiveness acceptability (ICEA) curves for concerns about falls

On the left the CE-planes for concerns about falls (FES-I as outcome).

On the right the ICEA-curves for AMB-Home, with on the $y$-axis the probability that the program is costeffective and on the $x$-axis the willingness to pay given various ceiling ratios with res pect to concerns about falls. Sensitivity analyses performed for per-protocol, healthcare perspective, without outliers, and activity avoidance.

\section{Sensitivity analyses}

As the cost-effectiveness of the program may vary depending on the groups, perspective and outcomes that are chosen, sensitivity analyses were performed by selecting participants on the basis of their exposure to the program (per-protocol), from a healthcare perspective and after deleting extreme outliers. In Table 3, ICERs and the distribution of the ICERs on the CE-planes are presented for these sensitivity analyses, both for concerns about falls and QALYs as outcomes. Overall, the probability of the cost-effectiveness of AMB-Home increased if participants received five or more sessions 
compared to usual care, decreased when only costs were taken from a healthcare perspective, and was rather similar without outliers to the base case analyses. In Figure 2 the CE-planes and ICEA-curves for concerns about falls showed that the probability that the program was cost-effective was $99 \%$ at a WTP of 600 Euro for one point increase on the FES-I for those who received five or more sessions (this line does not reach the $100 \%)$. And from a healthcare perspective the program was $100 \%$ cost-effective at a WTP of 1,300 Euro.

With respect to QALYs, the probability that the program was cost-effective with a WTP of 20,000 Euro was $87 \%$ if participants received five or more sessions (Figure 3 ). With the same WTP of 20,000 and analyzed from a healthcare perspective, the probability of cost-effectiveness of AMB-Home was $73 \%$.

The range of the probability of cost-effectiveness for avoidance behavior due to concerns about falls was almost the same as for the base case analyses of concerns about falls (Figure 2).

\section{Base case}

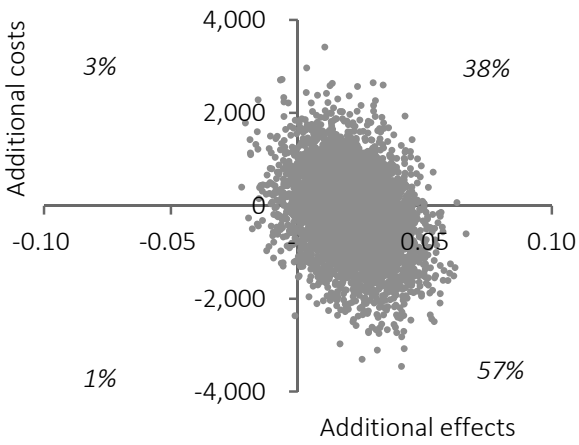

Per-protocol

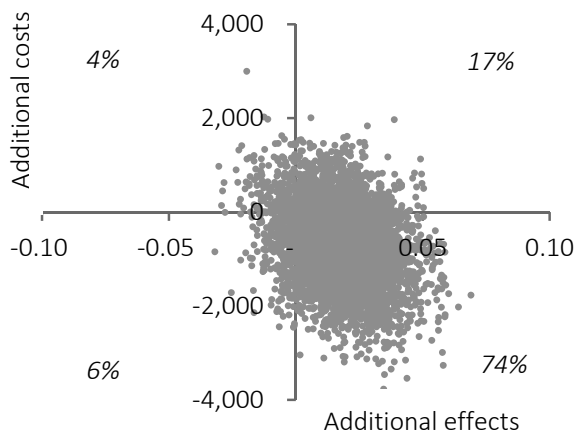

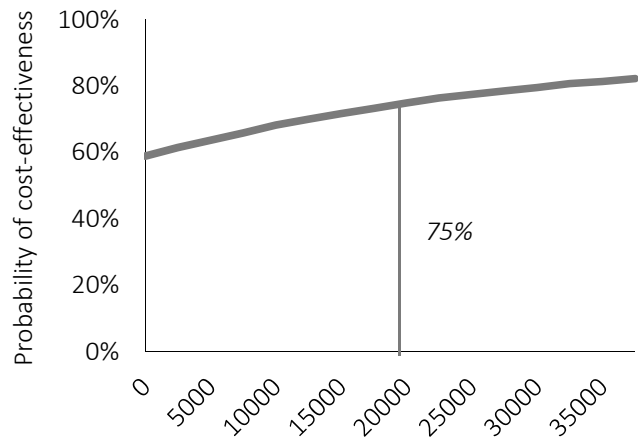

Willingness to pay; $€$ / point on T-score

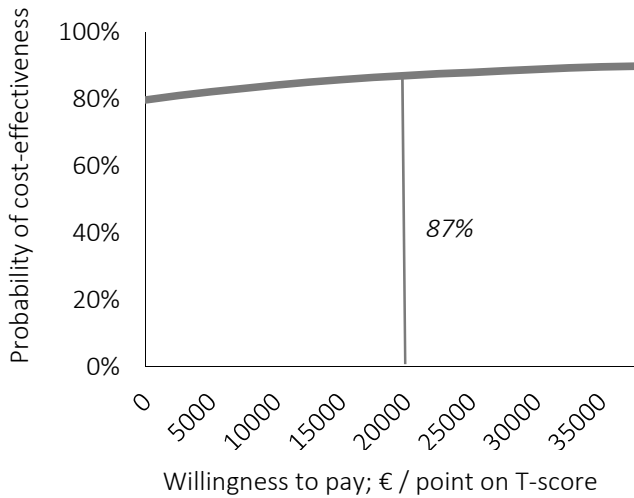




\section{Healthcare perspective}

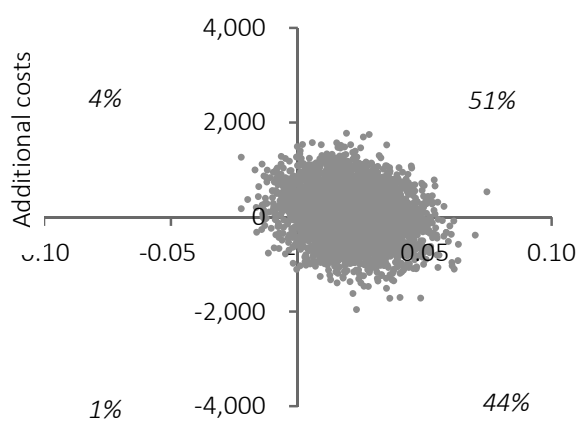

Additional effects

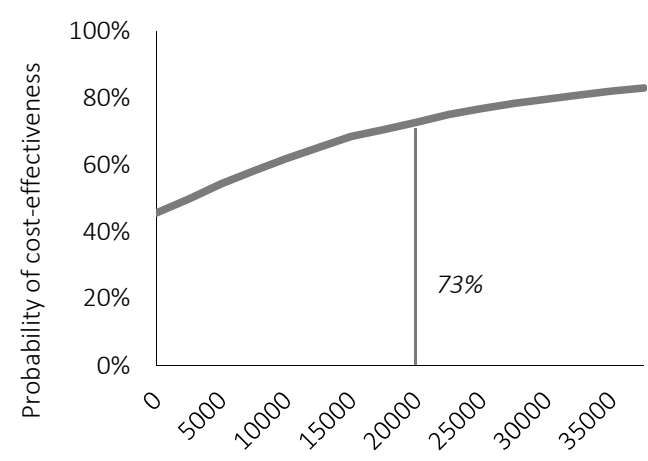

Willingness to pay; $€ /$ point on T-score

Without outliers
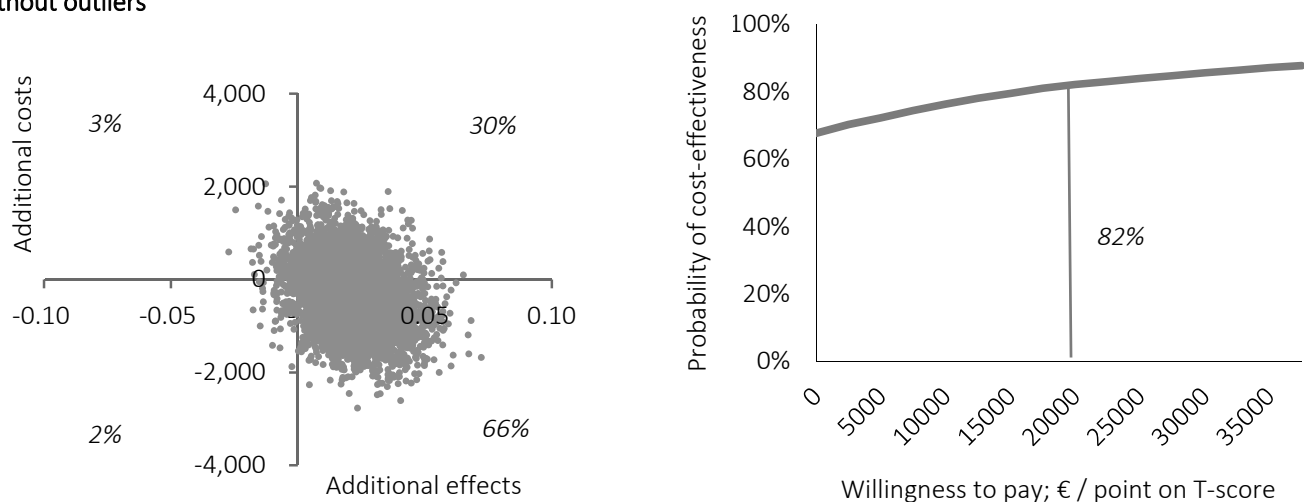

Figure 3 Cost-effectiveness planes (CE-planes) and incremental cost-effectiveness acceptability (ICEA) curves for QALYS

On the left the CE-planes for QALYS (SF-6D classification index as outcome).

On the right the ICEA-curves for AMB-Home, with on the $y$-axis the probability that the program is costeffective and on the $x$-axis the willingness to pay given various ceiling ratios with respect to QALYs. Sensitivity analyses performed for per-protocol, healthcare perspective, without outliers.

\section{Discussion}

In this study we assessed the cost-effectiveness of AMB-Home, a home-based, cognitive behavioral program to reduce concerns about falls from a societal perspective. Total societal costs were 8,094 Euro in the usual care group and 7,890 Euro in the intervention group, including 716 Euro for the program. With regard to concerns about falls as well as QALYs, the program is likely to be cost-effective. For QALYS the probability is 
$75 \%$ that the program is cost-effective at a WTP of 20,000 Euro. For concerns about falls the probability is already $100 \%$ with a WTP of 1,300 Euro. Sensitivity analyses showed that if participants received at least five out of the seven sessions the probability that AMB-Home is cost-effective increased. When costs were taken from a healthcare perspective the probability of AMB-Home being a cost-effective program decreased.

Previously, studies were conducted to examine the potential cost savings of the U.S. version of 'A Matter of Balance'. ${ }^{25,26}$ However, as far as we know, only the Dutch group program of a $\mathrm{AMB}$ reported on the costs related to the effectiveness in the domain of concerns about falls. ${ }^{23}$ As the study of van Haastregt and colleagues did not use QALYs nor the FES-I as effect outcome measure it is difficult to compare this study with our study. However, all economic evaluations of the program in the US or the Netherlands were preferable in terms of costs and effects when compared to usual care.

In our calculation the probability that the intervention is cost-effective looking at concerns about falls was $100 \%$ at a WTP of 1,300 Euro. It is however hard to determine the amount of Euros that the society is WTP for concerns about falls among older people. This is a common difficulty for disease specific instruments. ${ }^{57,58}$ Future research aimed at identifying maximum WTP thresholds for commonly used outcomes related to functional and psychological outcomes in older people is warranted. Accordingly, clinical effects can be better compared to QALYs in economic evaluations.

Several limitations of the present study should be noted. First, dropout rates of study participants were substantial and different in both study groups, i.e. $17 \%$ in the usual care group and 34\% in the intervention group. Therefore, selective dropout may be an issue, although additional analyses showed no significant differences between dropouts in both groups with respect to the selected baseline characteristics. Second, the withdrawal from AMB-Home was high, i.e. $40 \%$ of the participants in the intervention group received less than five of seven sessions. ${ }^{59}$ Third, we were not able to adjust for potential baseline differences with respect to costs between both groups, because costs were not measured at baseline. As a result, differences in costs from the beginning of the study between the groups could have influenced the final results. Lastly, the timeframe of one year is relatively short for an economic evaluation. Therefore, it remains unclear whether the positive effects of the program maintain on the long run.

Despite these limitations, to our current knowledge this is the first study comparing not only the cost-effectiveness, but also the cost-utility of the cognitive behavioral program 'A Matter of Balance'. ${ }^{23,25,26}$ Strengths of this economic evaluation were strong study design, including longitudinal, prospective data collection, and costs taken from a societal perspective in a frail population.

The adherence to AMB-Home was not optimal, while, the outcomes of the perprotocol sensitivity analyses revealed that the cost-effectiveness of the intervention is considerably higher for the group who received at least five sessions. Therefore, it should be explored if a personal intake ${ }^{31}$, and tailoring the number of sessions of the 
program to the capacities, skills and preferences of the participant ${ }^{60}$ can lead to a higher adherence rate and to better results on effectiveness and costs.

\section{Conclusions}

For frail older people who are concerned about falling, AMB-Home can be considered as good value for money, because the program has reasonable probability of being costeffective. AMB-Home demonstrated no effects on quality of life, but clinical effects were shown for concerns about falls and there was a small decrease in costs. Therefore, this home-based, individualized AMB format is a welcome addition to current geriatric care, particularly for those persons who are not able or willing to attend group programs. 


\section{References}

1. Scheffer AC, Schuurmans MJ, van Dijk N, van der Hooft T, de Rooij SE: Fear of falling: measurement strategy, prevalence, risk factors and consequences among older persons. Age Ageing. 2008, 37(1):19-24.

2. Legters K: Fear of falling. Phys Ther. 2002, 82(3):264-272.

3. Zijlstra GA, van Haastregt JC, van Eijk JT, van Rossum E, Stalenhoef PA, Kempen GI: Prevalence and correlates of fear of falling, and associated avoidance of activity in the general population of communityliving older people. Age Ageing. 2007, 36(3):304-309.

4. Li F, Fisher KJ, Harmer P, McAuley E, Wilson NL: Fear of falling in elderly persons: association with falls, functional ability, and quality of life. J Gerontol B Psychol Sci Soc Sci. 2003, 58(5):P283-290.

5. Kressig RW, Wolf SL, Sattin RW, O'Grady M, Greenspan A, Curns A, Kutner M: Associations of demographic, functional, and behavioral characteristics with activity-related fear of falling among older adults transitioning to frailty. J Am Geriatr Soc. 2001, 49(11):1456-1462.

6. Deshpande N, Metter EJ, Lauretani F, Bandinelli S, Guralnik J, Ferrucci L: Activity restriction induced by fear of falling and objective and subjective measures of physical function: a prospective cohort study. $J$ Am Geriatr Soc. 2008, 56(4):615-620.

7. Cumming RG, Salkeld G, Thomas M, Szonyi G: Prospective study of the impact of fear of falling on activities of daily living, SF-36 scores, and nursing home admission. J Gerontol A Biol Sci Med Sci. 2000, 55(5):M299-305.

8. Boyd R, Stevens JA: Falls and fear of falling: burden, beliefs and behaviours. Age Ageing. 2009, 38(4):423-428.

9. Delbaere K, Close JC, Brodaty H, Sachdev P, Lord SR: Determinants of disparities between perceived and physiological risk of falling among elderly people: cohort study. BMJ. 2010, 341:c4165.

10. Denkinger MD, Lukas A, Nikolaus T, Hauer K: Factors associated with fear of falling and associated activity restriction in community-dwelling older adults: a systematic review. Am J Geriatr Psychiatry. 2015, 23(1):72-86.

11. Clemson L, Kendig H, Mackenzie L, Browning C: Predictors of injurious falls and fear of falling differ: an 11-year longitudinal study of incident events in older people. J Aging Health. 2015, 27(2):239-256.

12. Zijlstra GA, van Haastregt JC, van Rossum E, van Eijk JT, Yardley L, Kempen GI: Interventions to reduce fear of falling in community-living older people: a systematic review. J Am Geriatr Soc. 2007, 55(4):603-615.

13. Bula CJ, Monod S, Hoskovec C, Rochat S: Interventions aiming at balance confidence improvement in older adults: an updated review. Gerontology. 2011, 57(3):276-286.

14. Kendrick D, Kumar A, Carpenter H, Zijlstra GA, Skelton DA, Cook JR, Stevens Z, Belcher CM, Haworth D, Gawler SJ et al: Exercise for reducing fear of falling in older people living in the community. Cochrane $D b$ Syst Rev. 2014, 11.

15. Peterson EW: Using cognitive behavioral strategies to reduce fear of falling: A matter of balance. Generations. 2002, 26(4):53-59.

16. Zijlstra GA, Tennstedt SL, van Haastregt JC, van Eijk JT, Kempen GI: Reducing fear of falling and avoidance of activity in elderly persons: the development of a Dutch version of an American intervention. Patient Educ Couns. 2006, 62(2):220-227.

17. Tennstedt S, Howland J, Lachman M, Peterson E, Kasten L, Jette A: A randomized, controlled trial of a group intervention to reduce fear of falling and associated activity restriction in older adults. J Gerontol B Psychol Sci Soc Sci. 1998, 53(6):P384-392.

18. Zijlstra GA, van Haastregt JC, Ambergen T, van Rossum E, van Eijk JT, Tennstedt SL, Kempen Gl: Effects of a multicomponent cognitive behavioral group intervention on fear of falling and activity avoidance in community-dwelling older adults: results of a randomized controlled trial. J Am Geriatr Soc. 2009, 57(11):2020-2028.

19. Zijlstra GA, van Haastregt JC, van Eijk JT, de Witte LP, Ambergen T, Kempen GI: Mediating effects of psychosocial factors on concerns about falling and daily activity in a multicomponent cognitive behavioral group intervention. Aging Ment Health. 2011, 15(1):68-77. 
20. Batra A, Melchior M, Seff L, Frederick N, Palmer RC: Evaluation of a community-based falls prevention program in South Florida, 2008-2009. Prev Chronic Dis. 2012, 9:E13.

21. Page TF, Batra A, Palmer R: Cost analysis of a community-based fall prevention program being delivered in South Florida. Fam Community Health. 2012, 35(3):264-270.

22. Smith ML, Jiang L, Ory MG: Falls efficacy among older adults enrolled in an evidence-based program to reduce fall-related risk: sustainability of individual benefits over time. Fam Community Health. 2012, 35(3):256-263.

23. van Haastregt JC, Zijlstra GA, Hendriks MR, Goossens ME, van Eijk JT, Kempen GI: Cost-effectiveness of an intervention to reduce fear of falling. Int J Technol Assess Health Care. 2013, 29(3):219-226.

24. Cho J, Smith ML, Ahn S, Kim K, Appiah B, Ory MG: Effects of an evidence-based falls risk-reduction program on physical activity and falls efficacy among oldest-old adults. Front Public Health. 2014, 2:182.

25. Howland J, Shankar KN, Peterson EW, Taylor AA: Savings in acute care costs if all older adults treated for fall-related injuries completed matter of balance. Inj Epidemiol. 2015, 2(1):25.

26. Ghimire E, Colligan EM, Howell B, Perlroth D, Marrufo G, Rusev E, Packard M: Effects of a communitybased fall management program on medicare cost savings. Am J Prev Med. 2015, 49(6):e109-116.

27. Chen T, Edwards JD, Janke MC: The Effects of the A Matter of Balance program on falls and physical risk of falls, Tampa, Florida, 2013. Prev Chronic Dis. 2015, 12:150096.

28. Healy TC, Peng C, Haynes MS, McMahon EM, Botler JL, Gross L: The feasibility and effectiveness of translating a matter of balance into a volunteer lay leader model. J App/ Gerontol. 2008, 27(1):34-51.

29. Ory MG, Smith ML, Wade A, Mounce C, Wilson A, Parrish R: Implementing and disseminating an evidence-based program to prevent falls in older adults, Texas, 2007-2009. Prev Chronic Dis. 2010, 7(6):A130.

30. Ullmann G, Williams HG, Plass CF: Dissemination of an evidence-based program to reduce fear of falling, South Carolina, 2006-2009. Prev Chronic Dis. 2012, 9:E103.

31. Zijlstra GA, van Haastregt JC, Du Moulin MF, de Jonge MC, van der Poel A, Kempen Gl: Effects of the implementation of an evidence-based program to manage concerns about falls in older adults. Gerontologist. 2013, 53(5):839-849.

32. van Haastregt JC, Zijlstra GA, van Rossum E, van Eijk JT, de Witte LP, Kempen GI: Feasibility of a cognitive behavioural group intervention to reduce fear of falling and associated avoidance of activity in community-living older people: a process evaluation. BMC Health Serv Res. 2007, 7:156.

33. Dorresteijn TA, Rixt Zijlstra GA, Van Eijs YJ, Vlaeyen JW, Kempen Gl: Older people's preferences regarding programme formats for managing concerns about falls. Age Ageing. 2012, 41(4):474-481.

34. Dorresteijn TA, Zijlstra GA, Delbaere K, van Rossum E, Vlaeyen JW, Kempen GI: Evaluating an in-home multicomponent cognitive behavioural programme to manage concerns about falls and associated activity avoidance in frail community-dwelling older people: design of a randomised control trial [NCT01358032]. BMC Health Serv Res. 2011, 11:228.

35. Dorresteijn TA, Zijlstra GA, Ambergen AW, Delbaere K, Vlaeyen JW, Kempen GI: Effectiveness of a homebased cognitive behavioral program to manage concerns about falls in community-dwelling, frail older people: results of a randomized controlled trial. BMC Geriatr. 2016, 16(1):2.

36. Harper S: Economic and social implications of aging societies. Science. 2014, 346(6209):587-591.

37. Oostenbrink JB, Bouwmans CAM, Koopmanschap MA, FFH. R: Handleiding voor kosten onderzoek; methoden en standaard kostprijzen voor economische evaluaties in de gezondheidszorg (Geactualiseerde versie 2010). Diemen: The Netherlands: College voor Zorgverzekeringen; 2010.

38. Husereau D, Drummond M, Petrou S, Carswell C, Moher D, Greenberg D, Augustovski F, Briggs AH, Mauskopf J, Loder E et al: Consolidated health economic evaluation reporting Standards (CHEERS)-explanation and elaboration: a report of the ISPOR health economic evaluation publication guidelines good reporting practices task force. Value Health. 2013, 16(2):231-250.

39. Stewart AL, Hays RD, Ware JE, Jr.: The MOS short-form general health survey. Reliability and validity in a patient population. Med Care. 1988, 26(7):724-735. 
40. Vestjens L, Kempen GI, Crutzen R, Kok G, Zijlstra GA: Promising behavior change techniques in a multicomponent intervention to reduce concerns about falls in old age: a Delphi study. Health Educ Res. 2015, 30(2):309-322.

41. Dorresteijn TAC, Zijlstra GAR, Ambergen AW, Delbaere K, Vlaeyen JWS, Kempen GIJM: Effects of an InHome Cognitive Behavioral Program to Manage Concerns About Falls in Community-Dwelling Frail Older People: Results of a Randomized Controlled Trial. submitted.

42. Kempen GI, Zijlstra GA, van Haastregt JC: [The assessment of fear of falling with the falls efficacy scaleinternational (FES-I). Development and psychometric properties in Dutch elderly]. Tijdschr Gerontol Geriatr. 2007, 38(4):204-212.

43. Yardley L, Beyer N, Hauer K, Kempen G, Piot-Ziegler C, Todd C: Development and initial validation of the falls efficacy scale-international (FES-I). Age Ageing. 2005, 34(6):614-619.

44. Zijlstra G, Dorresteijn T, Vlaeyen J, Kempen GI: Measuring avoidance behavior due to fear of falling in community-living older adults. Gerontologist. 2013, 53:199-199.

45. Gandek B, Ware JE, Aaronson NK, Apolone G, Bjorner JB, Brazier JE, Bullinger M, Kaasa S, Leplege A, Prieto $L$ et al: Cross-validation of item selection and scoring for the SF-12 health survey in nine countries: results from the IQOLA Project. International quality of life assessment. J Clin Epidemiol. 1998, 51(11):1171-1178.

46. Brazier JE, Roberts J: The estimation of a preference-based measure of health from the SF-12. Med Care. 2004, 42(9):851-859.

47. Drummond MF: Methods for the economic evaluation of health care programmes, 3rd edn. Oxford: Oxford University Press; 2005.

48. Personal communication with QualityMetric. October 2012.

49. Goossens MEJB, Rutten-van Molken MPH, Vlaeyen JWS, van der Linden SMJP: The cost diary: a method to measure direct and indirect costs in cost-effectiveness research. J Clin Epidemiol. 2000, 53(7):688-695.

50. Raad voor de Volksgezondheid en Zorg: Zinnige en duurzame zorg: transparante keuzen in de zorg voor een houdbaar zorgstelsel. Zoetermeer: Raad voor de Volksgezondheid en Zorg; 2006.

51. Zorginstituut Nederland: Kosteneffectiviteit in de praktijk. Diemen: Zorginstituut Nederland; 2015.

52. Mclnnes E, Seers K, Tutton L: Older people's views in relation to risk of falling and need for intervention: a meta-ethnography. J Adv Nurs. 2011, 67(12):2525-2536.

53. NIST/SEMATECH e-handbook of statistical methods [http://www.itl.nist.gov/div898/handbook/prc/ section1/prc16.htm]

54. Sinharay S, Stern HS, Russell D: The use of multiple imputation for the analysis of missing data. Psychol Methods. 2001, 6(4):317-329.

55. Briggs AH, Wonderling DE, Mooney CZ: Pulling cost-effectiveness analysis up by its bootstraps: a nonparametric approach to confidence interval estimation. Health Econ. 1997, 6(4):327-340.

56. Fenwick E, Byford S: A guide to cost-effectiveness acceptability curves. Br J Psychiatry. 2005, 187:106-108.

57. Clark F, Jackson J, Carlson M, Chou C-P, Cherry BJ, Jordan-Marsh M, Knight BG, Mandel D, Blanchard J, Granger DA et al: Effectiveness of a lifestyle intervention in promoting the well-being of independently living older people: results of the Well Elderly 2 randomised controlled trial. J Epidemiol Community Health. 2012, 66(9):782-790.

58. Dear BF, Zou JB, Ali S, Lorian CN, Johnston L, Sheehan J, Staples LG, Gandy M, Fogliati VJ, Klein B et al: Clinical and cost-effectiveness of therapist-guided internet-delivered cognitive behavior therapy for older adults with symptoms of anxiety: a randomized controlled trial. Behav Ther. 2015, 46(2):206-217.

59. Dorresteijn TA, Rixt Zijlstra GA, Van Haastregt JC, Vlaeyen JW, Kempen GI: Feasibility of a nurse-led inhome cognitive behavioral program to manage concerns about falls in frail older people: a process evaluation. Res Nurs Health. 2013, 36(3):257-270.

60. Simek EM, McPhate L, Hill KD, Finch CF, Day L, Haines TP: What are the characteristics of home exercise programs that older adults prefer?: A cross-sectional study. Am J Phys Med Rehabil. 2015, 94(7):508-521. 
CHAPTER 6

Additional file 1 Health care utilization: costs prices used

\begin{tabular}{|c|c|c|c|}
\hline \multirow{2}{*}{$\begin{array}{l}\text { Category } \\
\underline{\text { Intervention costs }}\end{array}$} & \multirow[t]{2}{*}{ Subcategory } & \multicolumn{2}{|c|}{ Costs in euros } \\
\hline & & $\begin{array}{l}\text { per } \\
\text { participant }\end{array}$ & $\begin{array}{l}\text { mean of } 26 \\
\text { participants }\end{array}$ \\
\hline Training (from Trimbos Institute) & $\mathrm{N} / \mathrm{A}$ & 302.00 & 12.00 \\
\hline $\begin{array}{l}\text { Intervention (training, preparation \& contact } \\
\text { hours) }\end{array}$ & Hours & 2041.00 & 683.00 \\
\hline $\begin{array}{l}\text { Materials (DVD, DVD-player, facilitator } \\
\text { workbook \& participant workbook) }\end{array}$ & $\mathrm{N} / \mathrm{A}$ & 111.00 & 21.00 \\
\hline Total & & 2455.00 & 716.00 \\
\hline \multicolumn{4}{|l|}{$\underline{\text { Healthcare costs }}$} \\
\hline \multicolumn{4}{|l|}{ Primary care } \\
\hline \multirow[t]{3}{*}{ GP and practice nurse } & Consultation & \multicolumn{2}{|l|}{29.00} \\
\hline & Telephone consultation & \multicolumn{2}{|l|}{15.00} \\
\hline & Home visit & \multicolumn{2}{|l|}{45.00} \\
\hline Occupational therapist & Session & \multicolumn{2}{|l|}{22.00} \\
\hline \multirow[t]{2}{*}{ Physiotherapist } & Session & \multicolumn{2}{|l|}{37.00} \\
\hline & Session at home & \multicolumn{2}{|l|}{53.00} \\
\hline \multirow[t]{2}{*}{ Other paramedics } & Session & \multicolumn{2}{|c|}{ From 23.00 till 80.00} \\
\hline & Session at home & \multicolumn{2}{|c|}{ From 38.00 till 96.00} \\
\hline \multicolumn{4}{|l|}{ Hospital care } \\
\hline Outpatient medical services & Consultation & \multicolumn{2}{|l|}{66.00} \\
\hline Day care & Day & \multicolumn{2}{|l|}{261.00} \\
\hline Hospital admission & Admission day & \multicolumn{2}{|l|}{452.00} \\
\hline \multicolumn{4}{|l|}{$\underline{\text { Patient and family costs }}$} \\
\hline Professional home care & Hour & \multicolumn{2}{|l|}{46.00} \\
\hline Professional domestic help & Hour & \multicolumn{2}{|l|}{25.00} \\
\hline Domestic help & Hour & \multicolumn{2}{|l|}{13.00} \\
\hline Informal care & Hour & \multicolumn{2}{|l|}{13.00} \\
\hline Helping aids/ in-home modifications & Number & \multicolumn{2}{|c|}{ From 9.00 till 2694.00} \\
\hline
\end{tabular}

Most prices are obtained from the Dutch manual for cost-analysis in healthcare research (2009), except helpings aids and in home modifications (internet, 2013); Subsequently, costs per categories were indexed to the reference year (2011) using a consumer price index. 
Additional file 2 Mean (SD) volumes of healthcare utilization 0-12 months

\begin{tabular}{|c|c|c|c|c|c|c|c|c|c|c|}
\hline \multirow[b]{2}{*}{ Care category } & \multicolumn{5}{|c|}{ Usual care group ( $n=159)$} & \multicolumn{5}{|c|}{ Intervention group $(n=130)$} \\
\hline & Mean & (SD) & Median & Min & Max & Mean & (SD) & Median & Min & Max \\
\hline \multicolumn{11}{|l|}{ Primary care } \\
\hline $\begin{array}{l}\text { General practitioner } \\
\text { (contacts) }\end{array}$ & 12.7 & $(9.7)$ & 11 & 0 & 63 & 11.4 & $(8.5)$ & 10 & 0 & 46 \\
\hline Consultation & 6.5 & $(6.3)$ & 5 & 0 & 51 & 6.5 & $(6.0)$ & 5 & 0 & 45 \\
\hline Telephone consultation & 4.1 & $(4.6)$ & 2 & 0 & 22 & 3.3 & $(4.0)$ & 2 & 0 & 23 \\
\hline Home visit & 2.1 & $(4.2)$ & 0 & 0 & 24 & 1.6 & $(2.7)$ & 0 & 0 & 12 \\
\hline Physiotherapy (contracts) & 22.6 & $(31.8)$ & 8 & 0 & 144 & 23.7 & $(28.1)$ & 12.5 & 0 & 112 \\
\hline Other therapists (contacts) & 2.6 & $(6.6)$ & 0 & 0 & 51 & 3.8 & $(13.3)$ & 0 & 0 & 104 \\
\hline \multicolumn{11}{|l|}{ Hospital care } \\
\hline Admission (days) & 2.2 & $(7.6)$ & 0 & 0 & 76 & 2.4 & $(8.3)$ & 0 & 0 & 56 \\
\hline Medical specialists (contacts) & 7.1 & $(6.6)$ & 5 & 0 & 33 & 6.9 & $(7.1)$ & 5 & 0 & 44 \\
\hline \multicolumn{11}{|l|}{ Patient and family } \\
\hline $\begin{array}{l}\text { Professional home care } \\
\text { (hours) }\end{array}$ & 22.4 & $(59.3)$ & 0 & 0 & 337 & 12.9 & $(38.4)$ & 0 & 0 & 247 \\
\hline $\begin{array}{l}\text { Professional domestic help } \\
\text { (hours) }\end{array}$ & 95.4 & $(108.6)$ & 27 & 0 & 364 & 83.8 & $(100.3)$ & 0 & 0 & 312 \\
\hline Domestic help (hours) & 30.5 & $(65.8)$ & 0 & 0 & 324 & 31.7 & $(62.1)$ & 0 & 0 & 260 \\
\hline Informal care (hours) & 68.5 & $(165.6)$ & 5 & 0 & 1340 & 50.6 & (99.9) & 5 & 0 & 540 \\
\hline Home adaptations (number) & 0.5 & $(1.3)$ & 0 & 0 & 9 & 0.5 & $(1.1)$ & 0 & 0 & 6 \\
\hline $\begin{array}{l}\text { Aids and assistive devices } \\
\text { (number) }\end{array}$ & 1.2 & $(1.5)$ & 1 & 0 & 9 & 1.2 & $(1.3)$ & 1 & 0 & 6 \\
\hline
\end{tabular}



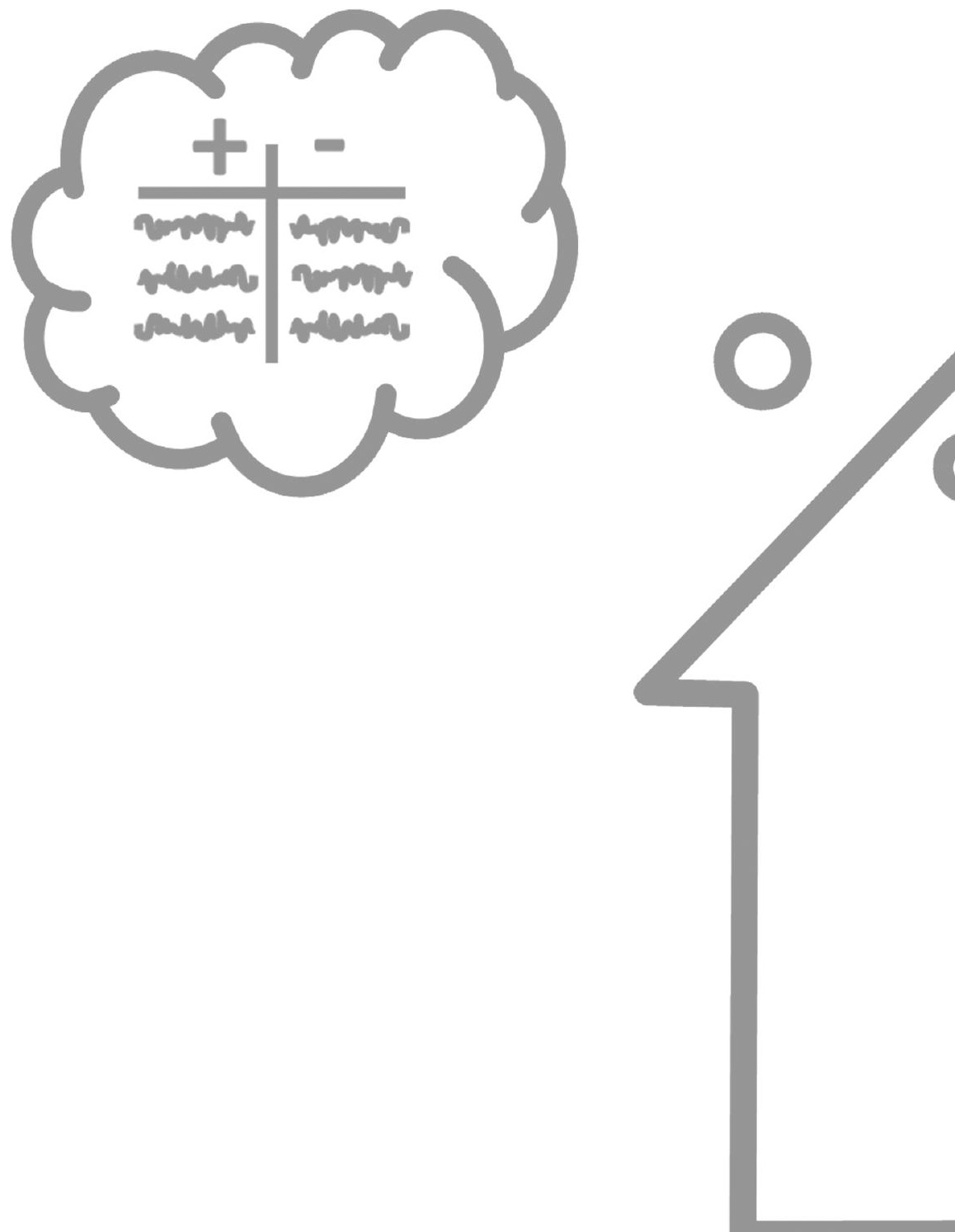
Chapter 7

Discussion 



\section{Introduction}

Concerns about falls are a common health problem and a threat to autonomy for community-dwelling older people. ${ }^{1}$ These concerns are associated with adverse outcomes in psychosocial, physical and functional domains such as, falls, avoidance of activity, a decreased balance performance, decreased mobility, lower social participation, functional decline, low quality of life, and loss of independence. ${ }^{1-6}$ In addition to the negative consequences of concerns about falls, there is the risk of increased healthcare usage and related costs. ${ }^{2}$ Reviews showed that a few approaches are successful in reducing concerns about falls $s^{7-9}$ and potentially contributors to a better quality of life and independent living of older people. The multicomponent, cognitive behavioral group program 'A Matter of Balance' (AMB) is one of the these few interventions that targets psychosocial, physical and functional aspects of concerns about falls and consistently shows positive effects on a broad range of outcomes. ${ }^{10-14}$ Despite its success in effectiveness, it seems that a substantial number of eligible participants do not attend or complete the group program because of health problems. ${ }^{11,12}$ To enable these frail, community-dwelling older people to participate, a tailor-made, home-based format, named 'A Matter of Balance at Home' (AMB-Home) was developed. AMB-Home was aimed at instilling adaptive and realistic views about falls, as well as increasing activity and safe behavior, and consisted of seven sessions, comprising three home visits and four telephone contacts. The program was facilitated by community nurses who were qualified in the field of geriatrics and worked at local home-care agencies.

This thesis had three main objectives. First, it aimed to explore which format(s) are preferred to manage concerns about falls by community-dwelling older people. Second, to study the feasibility and acceptability of a home-based, cognitive behavioral program to manage concerns about falls in frail, community-dwelling, older people. Third, to analyze whether the newly developed program is (cost-)effective with respect to the reduction of concerns about falls when compared to care as usual. In this final chapter the main findings of the thesis are summarized. Moreover, several methodological and theoretical issues will be discussed, and the chapter will end with some implications for clinical practice and suggestions for future research.

\section{Main findings}

\section{Preferences}

A cross-sectional study was performed to explore the preferences of communitydwelling older people regarding six different formats (in a group, at home, via telephone, via a combination of home visits and telephone consultations, via television or via internet) of a program that primarily focuses on managing concerns about falls (Chapter 2). Of the 2,498 responders, $62.7 \%$ indicated having no interest in any of the 
formats. The willingness to participate varied per program format and ranged from $21.5 \%$ (at home) to $9.4 \%$ (via internet). Higher levels of fall-related concerns were associated with increased preference for a program including home visits. Poor perceived health and older age ( $\geq 80$ years) were associated with less preference for a group program.

\section{Development of the program}

Eight experts in the domain of program development and behavioral change techniques were consulted in the development process of the home-based version for frail older people and a pilot study with six older volunteers was conducted to test the program's initial feasibility. This process resulted in a program that consists of seven individual sessions, including three home-visits (60,60 and 75 minutes, respectively) and four telephone contacts (35 minutes each) (Chapter 3). During each session, one main theme is addressed. The themes of the program are: concerns about falls; thoughts about falling; physical exercise; asserting oneself; overcoming personal barriers; safe behavior; and managing concerns about falls. Each session has a similar structure with the following sequence of elements: reviewing the previous session (except the first one); discussing the current session's theme; and formulating an action plan related to the theme that was discussed and that comprises a personal, tailor-made activity. Session 5 differed slightly from the other sessions in that participants were guided to safely execute a daily activity they were afraid to perform independently ('exposure in vivo'). ${ }^{15}$ Examples of activities selected by participants included walking down the stairs or crossing a street. A variety of techniques was used to encourage active participation and behavior change, including a DVD on role modeling, problem solving, action planning, and motivational interviewing.

Overall, principles for behavior change and themes of the group program were maintained in AMB-Home. However in adapting the group program to a home-based program several changes were made. First, the physical exercises in the group program were replaced by 'exposure in vivo', ${ }^{15}$ because the appropriate and safe execution of these exercises could not be guaranteed due to the limited face-to-face contact. Second, motivational interviewing was incorporated to encourage internal motivation to change behavior and increase self-efficacy. ${ }^{16}$ Next, participants were encouraged to invite a significant other (e.g., a spouse, friend, or neighbor) to be present during the home visits. This person was instructed how they could support the participant throughout the program, for example helping with the homework or by performing the action plans between the sessions without taking over activities from the participant. Lastly, the eight group sessions were replaced by seven individual sessions.

\section{Feasibility}

A comprehensive process evaluation alongside the randomized controlled trial was carried out in the intervention group in order to improve our understanding of the feasibility and acceptability of the AMB-Home program (Chapter 4). The following elements 
were measured: participation of the target population in the program (reach); implementation of the program as planned (fidelity); participants' engagement in and receptivity to the program (dose received - exposure); participants' and facilitators' satisfaction with the program (dose received - satisfaction); and aspects of the program that need improvement prior to nationwide implementation (barriers). Data were collected from eight nurses (the facilitators of the program) and 194 participants. Of the 194 participants, 117 (60\%) completed the program (i.e., received at least five of the seven sessions). The main reason for not completing was that participants lost interest. According to the self-reports of facilitators the program was generally performed as planned. Of the most important program components (discussion about the themes, formulation of action plans and performance of a challenging activity) the discussion parts were performed best (80-88\%); action plans were formulated less often towards the end of the program (51-73\%); and the supervised execution of a specifically selected activity during which the participants experienced concerns about falls ('exposure in vivo') in session 5 was only performed half of the time. Based on audio recordings we concluded that the performance according to protocol of the facilitators was much lower compared to their self-reported data. A large majority of participants indicated that they had reached at least one personal goal and that they experienced benefits from the program in daily life. Also the performance of the facilitator was highly valued by the participants. Facilitators indicated as a barrier that more than one third of the 117 participants who completed the program should not have enrolled in the program as they considered them not the target sample, for example because of physical limitations or not being restricted in daily activities.

\section{(Cost-)effectiveness}

A randomized controlled trial was conducted to examine the (cost-)effectiveness of the AMB-Home program. After screening with a written, postal questionnaire in a general population of community-dwelling older people of 70+, 398 participants were included in the study, who reported at least some concerns about falls and associated activity avoidance, and who perceived their general health as fair or poor. The mean age was 78.3 years and of those included was 70\% female. Through randomization, 195 participants were allocated to the control group, and 194 participants were included in the intervention group. Data were collected from 172 control and 139 intervention participants directly after the intervention and from 162 control and 133 intervention participants at 12 months follow-up. Main reasons for lost follow-up were similar in both groups, namely health problems and lost interest. Dropout rates in the intervention group were substantial higher than in the control group. Mixed-effects linear regression analyses showed significant lower levels of concerns about falls in the intervention group at 12 months compared with the control group (Chapter 5). Furthermore, significant reductions in self-reported activity avoidance, restrictions in daily life and indoor falls were identified in the intervention group compared with the control group. Effect 
sizes were small to medium. No significant difference in total number of falls was noted between the groups.

The economic evaluation was embedded in the trial and performed from a societal perspective with a time horizon of 12 months (Chapter 6). The main outcome measure for the cost-effectiveness (CEA) was concerns about falls and the outcome for the costutility analysis (CUA) was Quality Adjusted Life Years (QALY). The total societal costs for the usual care group is 8,094 Euro and 7,890 Euro in the intervention group of which the program costs were 716 Euro per participant. Total costs and subcategories of costs were comparable between the two groups. After synthesizing the effects and costs, the probability was substantially that the program was cost-effective for concerns about falls as well as for QALYS as outcome. Additional sensitivity analyses showed that if participants received 5 or more sessions the probability that the program was costeffective increased. When costs were taken from a healthcare perspective the probability of the cost-effectiveness of the program decreased.

\section{Considerations}

In the following paragraph are some general reflections given on strengths and limitations of the different studies in this thesis to facilitate the interpretation of the results and comparison with other studies.

\section{Recruitment}

Recruitment strategy used in this study can have influence on the generalizability of the results. ${ }^{17}$ Screening for eligibility for the trial and collecting data about the preferences was performed in a general population of community-dwelling people of 70 years and older. People were randomly selected by municipal registry offices and received a short postal questionnaire. Approximately half of the 11,490 community-living older people who were approached returned the screening list $(52 \%, n=6,029)$. The non-responders were slightly older than the responders and more likely to be female. Older age and female gender is associated with more concerns about falls. Other general characteristics could not be obtained from the non-responders, because of strict policies of municipal registry offices regarding people's privacy.

Subsequently, 977 persons (16\%) of the 6,029 responders were eligible for participation, and of these eligible responders, 588 persons (60.2\%) declined participation. Finally, 389 persons (3.4\% of the 11,490 addressed people) were enrolled into the study. Those who declined participation were again slightly older, were less likely to live alone, had less fallen in the past 6 months and were less concerned than those who were included into the study. Among the responders, in contrast to the nonresponders, no difference was found in gender between those who declined participation and those who did not. It remains unclear what the influence was of the non- 
response and participation refusal on the findings. In the first step of the selection procedure the characteristics of the non-responders could be associated with more concerns about falls, while in the second step those who had higher levels of concerns agreed to participate in the study.

\section{Dropout from the study}

Dropout rates from the (cost-)effectiveness study were substantial and different in both groups, i.e. $17 \%$ in the control group and 34\% in the intervention group. Most participants became lost-follow up between the baseline measurement and the assessment directly after the intervention period. Additional analyses showed no significant differences in the two study groups between those who completed the trial and those who did not for the selected baseline characteristics such as age, gender, living situation, educational level, perceived general health, falls in the past 6 months, and chronic diseases, and for the primary and secondary outcomes. However a selection bias cannot be ruled out for characteristics that were not assessed in the study.

\section{Withdrawal from the program}

A substantial number of participants withdrew from the program early, the main reason being the loss of interest in the treatment. Maybe the inclusion procedure deserved more attention so that participants were better informed about the content of the program. Although people received a detailed leaflet with information about both the trial and the program, a personal intake may have worked better as was noted in a previous implementation study of the group program to reduce concerns about falls. ${ }^{13}$ Next, health problems and burden of the program were the second most important reason for withdrawal from the program. Like intended a more frail and disabled study sample was selected compared with samples in previous trials ${ }^{12,18}$, yet, more adaptions have to be made to enable this frailest group to complete the program. However, the adherence rate of $60 \%$ in our study did not differ substantially from other trials with individual multifactorial interventions with a range from 28 to $95 \%{ }^{19}$

Facilitators indicated that more than one third of the 117 participants $(n=41)$ who completed the program should not have enrolled in the program. The main reasons given for this assumption were physical limitations, not being restricted in daily activities, cognitive impairment, not motivated for the program, or not concerned about falling. Again, a more accurate screening procedure could have facilitated a better match between the program and qualified participants. However, there may have been a discrepancy between the participant's eligibility and the facilitator's perception of eligibility. For example, $85 \%$ of the participants reported benefits for daily life after completing the program. Also the levels of concerns about falls at baseline point out that more than $90 \%$ of the participants scored 23 points or higher on the Falls Efficacy Scale - International (FES-I; range 16-64), which is suggested as an indication for having substantial concerns about falls. ${ }^{20}$ 


\section{Treatment fidelity}

Opening the black box and examine whether the program was carried out as planned was one of the main objectives of our process evaluation. ${ }^{21,22}$ Therefore, facilitators were asked to report after each contact on the time spent per session and the extent to which the program was performed according to protocol. The mean duration of the sessions as reported by the facilitators was generally comparable with the time planned in the protocol. Also the performance according protocol was high according to the facilitators. Nevertheless, when we compare the fidelity scores of the facilitators with the fidelity scores based on audio recordings, there was a considerable gap between both outcomes. The self-reported adherence to the protocol by the facilitators was substantially higher compared to the independent ratings of the audio recordings. There may be several explanations for this discrepancy. First, despite our explicit statement that their answers would only be used for the process evaluation purposes, facilitators may have given socially desirable answers. Second, facilitators may not have been sufficiently aware of not following the protocol as intended. A third reason could be that facilitators had difficulty recalling the session when filling in the questionnaire afterwards. The findings and explanations suggest that using audio recordings is a valuable method to assess the treatment fidelity, because self-report is not always reliable. Moreover, video- or audio recordings can be used during implementation as a feedback tool to increase fidelity of the program as well as using behavior change counseling skills in a correct way. ${ }^{23-25}$

Another point of concern regarding the fidelity of the program was the formulation of action plans. This main component was less and less often carried out towards the end of the program. Also the performance of the (compared to the group program) newly added supervised challenging activity by the participant in session 5 ("gradual exposure to an activity one is fearful of") was not performed by almost one third of the participants. According to the facilitators, the main reason for not performing such an activity was the participant's inability to think of a suitable activity. Selecting suitable activities for the goal-setting and action-planning can be challenging. ${ }^{26}$ To overcome this difficulty, 16 pictures of the Icon-FES ${ }^{27}$, which include the daily activities used in the assessment of concerns about falls by the FES- ${ }^{28}$, were incorporated in session 2 and served as examples for the selection of activities by the participant. Nevertheless, in the future more attention should be given in session 2 to the selection of suitable and for the participant relevant activities. Successful performed activities by participants in our study together with the 30 activities of the full Icon-FES could serve as good examples. These example activities have then to be described in the facilitators' manual and extra attention could be given to goal setting during the facilitator training.

\section{Outcome measures}

As is the case in many studies in the domain of concerns about falls, collected data in our randomized study was based on self-report by means of telephone interviews. 
Questionnaires have relatively low costs and are therefore a common method for data collection in large-scale studies. Above and beyond, perceptions of people can only be measured by self-report. However, the use of self-report measurements has potential disadvantages like social desirable answers and recall bias. Some actions were taken to increase the validity of the outcome measures, even though no differences in social desirable answers or recall bias were expected between the two groups. First, mostly measures with good psychometric properties were used. Second, during the process evaluation, multiple sources were used to collect data, like besides self-reports, also audio tapes. Last, a monthly calendar was used for obtaining information about falls and healthcare use. Participants had to return the calendar sheets each month and were reminded by telephone if a calendar was not returned after 10 days to prevent missings and recall bias.

Although we have carefully chosen our outcome measures, co-led by practical reasons, adding some outcomes could have enabled us to contribute to a better understanding of the construct of concerns about falls. For instance no objective measurements were included to assess the physiological (e.g. physical reaction on a fear stimulus) and behavioral aspects (e.g. gait) of fear. Also potential mediating factors ${ }^{29}$ reflecting the potential underlying mechanisms of the program like daily activity ${ }^{30}$, fall related self-efficacy ${ }^{31}$, balance performance ${ }^{32}$ and personal goals ${ }^{33-35}$ were not included in the data collection. However, we have collected data on the following potential mediating factors: changes in perceived consequences of falling, catastrophic beliefs about consequences of a fall, perceived control over falling, mastery, feelings of anxiety, symptoms of depression and social support interactions. ${ }^{14,29,36,37}$ And outcome expectancies as potential moderator. ${ }^{38}$ Future analyses including these factors may give more in-depth information about contributors to the effects of our home-based cognitive behavioral program. In addition, a comparison can be made with the mechanisms underlying the group program and potential differences herein between the home-based and group format. $^{12,14}$

\section{Clinical relevance}

The results of our controlled trial showed a significant reduction of concerns about falls with a mean decrease of nearly 4 points on the FES-I (range 16-64). Yet, psychosocial outcomes are not unequivocal, like life versus death or being able to walk versus being paralyzed. Therefore it is not so easy to define what a change on a measurement scale means for participants. Effect sizes can indicate the magnitude of the change on the measurements that are used in trials, however, it is not an indication of a clinical relevant change. ${ }^{39}$ By using the Reliable Change Index ${ }^{40,41}$, it was possible to calculate a reliable change score on the FES-I of 9 points. If a participant had a change score of 9 points or higher, the participants' change was beyond the change which could be attributed to measurement error. Next step would be to calculate a Clinically Significant Change $^{40}$ by comparing the end state of the participant with the scores observed in 
socially and clinically meaningful comparison groups using norms. However, such norms are not available for concerns about falls. Delbaere and colleagues suggested cut-off points for the FES-I; nevertheless, before such cut-off points can be considered as the general norm, more research is needed as meaningful cut-off scores may vary across different samples and settings. ${ }^{20}$

Looking at which specific items of the FES-I people are less concerned to fall is another option for determining the clinical relevance of results of the program. Deshpande and colleagues showed in their study significant characteristic and functional differences between older people who had concerns about falls while performing activities in the community and those who expressed concerns in activities in and around their house. ${ }^{42}$ Concerns about falls in home environment activities were associated with older and frailer individuals who had lower personal mastery and social support, more depressive symptoms, and a lower functional performance. Or consider the participants' self-perceived benefits of the program as clinical relevant outcome, like was done in the process evaluation. This may give a good impression about what participants learned from the program, although, no comparison can be made between the control and intervention group participants.

\section{Effective components of the program}

$\mathrm{AMB}-\mathrm{Home}$ and the group program $\mathrm{AMB}-\mathrm{NL}$ are multifactorial programs, in which a number of behavior change techniques (BCTs) interact with each other. Despite the effectiveness of both $\mathrm{AMB}-\mathrm{Home}$ and $\mathrm{AMB}-\mathrm{NL}^{12}$, the effect sizes of the outcomes in both studies were only small to medium. Optimizing the intervention effects by concentrating on the most effective components is therefore warranted. In a Delphi study among 16 experts to reach consensus on the most promising BCTs of AMB-NL, 27 BCTs were identified, but goal setting, graded tasks and behavioral practice/rehearsal were pointed as most important and promising components. ${ }^{43}$ However, at the same time we found in the present study of AMB-Home that particularly these components were performed to a lesser degree. In order to improve the effects of the intervention, more efforts should be undertaken to integrate goal setting and subsequently graded tasks and rehearsal of the practiced behavior into the program. ${ }^{44,45}$ Moreover, other research designs than the standard randomized controlled trial are probably more appropriate to test the most effective components, for example randomized single case cross over trials ${ }^{46}$ or sequential multiple assignment randomized trials. ${ }^{47}$ Within these research designs there can be varied with the different components, time frames and modes of delivery. 


\section{Recommendations}

\section{Clinical practice and implementation}

Based on the results of the studies presented in this thesis several recommendations for clinical practice and implementation are suggested.

First, implementation of AMB-Home in the regular Dutch health care system is warranted given the favorable outcomes of the process- and (cost)effectiveness study. Many effective fall-related interventions remain unused in the wider community, therefore the gap between research outcomes and daily practice has to be bridged. ${ }^{48}$ The implementation study of AMB-NL based on a framework for implementing health care interventions ${ }^{49}$, can serve as a good example how a program to manage concerns about falls can be successfully disseminated nationwide. ${ }^{13}$ A face-to-face intake procedure, for example, does extensively reduce early withdrawal. ${ }^{13}$ Within this intake eligibility and motivation of potential participants for the program is checked and clear information about the program is provided to match expectancies. Furthermore, adherence and effectiveness can be improved through a better fit of personal reasons to participate ${ }^{50}$ and knowledge about readiness to change. ${ }^{51,52}$

Second, motivational interviewing was used as technique to encourage active participation and behavior change of the participant in the present study. Based on observations during the study and results from previous studies can be concluded that more training of the facilitators is needed to master this behavior change counseling skill. ${ }^{23-25}$ Facilitators suggested to provide an internet forum to share challenges and their solutions in conducting the program, but also extra training modules could be added and the option to give feedback on each other's audio- or videotaping in relation to motivational interviewing skills.

Third, to replace the social support from other participants, which is an important component in the group format, participants were encouraged to invite a significant other, for example, a spouse, other relative, friend, or neighbor. This resulted in an attendance rate of $25 \%$ of a significant other by the participants who completed the program. Facilitators were very positive about the role of the significant other in cases where someone was present. Based on these experiences more efforts on inviting a significant other could be recommended.

Fourth, the healthcare system in the Netherlands is still in transition. The responsibility of many healthcare tasks are moved from the national government to the local government and to healthcare agencies which offer opportunities for more customized prevention. ${ }^{53}$ And health is considered no longer as a state of total well-being, but the ability to adapt and to self-manage in the face of social, physical, and emotional challenges. ${ }^{54}$ AMB-Home fits perfectly with these new developments and should therefore be adopted and financially supported by healthcare agencies and local governments. Yet, older people also have their own responsibilities in managing their health therefore a small contribution would be in place, somewhere between $€ 50$ and $€ 150$, depending 
on their financial position. Several healthcare insurers now reimburse some costs for AMB-NL thru their additional packages.

Last, in the Netherlands telephone consultations in interventions are not commonly used in an older population. Community nurses who facilitated the program were sceptic at the start about the suitability of the telephone contacts in this target group. However, only eight of the 117 participants indicated the telephone contacts as a barrier. We can therefore recommend telephone contacts in combination with home visits as good alternative of exclusively home visits, especially with respect to the costeffectiveness.

\section{Future research}

Also several implications for future research are specified.

First, during the last decades of research in the area of concerns about falling, different terms were introduced accompanied with different measurements. ${ }^{28,31,55-57}$ The introduction of all these constructs of fall-related psychological concerns have led to confusion about which construct is meant in a study; instruments are often used to measure constructs other than for which the instruments were designed to assess. ${ }^{58}$ An example from Moore and colleagues ${ }^{58}$ : 'the Falls Efficacy Scale (FES), which was designed to measure the construct of falls efficacy, has been used extensively to measure fear of falling ${ }^{31}$, and the Activities-specific Balance Confidence (ABC) Scale, which was designed to measure the construct of balance confidence, has been used to measure fear of falling ${ }^{59}$ and fall-related self-efficacy.' Clear guidelines about which measure should be used to assess which construct of fall-related psychological concerns is needed. Moreover, consensus about a common outcome data set for the examination of fall-related psychological concerns and related factors would be a great step forward in the field of concerns about falls. With such common data set, outcomes of studies are better comparable and the mechanism underlying concerns about falls can be studied at a larger scale. A suggested data set of self-reported measures would include at least concerns about falls, fall-related self-efficacy, physical functioning, social participation, avoidance activity, and falls. Ideally this would be complemented with objective measures of gait, balance, muscle strength, vision, and proprioception.

Second, much is known about the risk factors associated with fall-related psychological constructs ${ }^{4}$, although much less is known about causal relationships between these risk factors and such outcomes. For instance, Hadjistavropoulos and colleagues demonstrated the weak causal relationship between activity avoidance and falls. ${ }^{60}$ Also, our study provides no answers to these questions. It seems plausible that past falls, beliefs, fall related self-efficacy, concerns about falls, social support, gait, activity avoidance, physical status, and future falls are interacting, but which comes first? Longitudinal studies on aging, wherein all these factors are included, are necessary to disentangle this issue. More knowledge about the offset and development of concerns about falls 
may help in establishing risk profiles and tailoring the programs to the needs of those who it regards.

Third, outcomes of comparable studies may support the external validity of our results and increase the evidence that an individual cognitive behavioral approach can contribute to the management of concerns about falls. The two following studies are rather similar to our study and carried out currently. First, Parry and colleagues have developed in the UK an individual cognitive behavioral therapy largely based on the group program a Matter of Balance. ${ }^{61}$ The program is now tested in a randomized control trial. Participants in the control group receive routine care in a fall clinic like all, and participants in the intervention group receive additionally 8 weekly sessions and a booster session at 6 months facilitated by a healthcare assistant. Second, Jayasinghe and colleagues have developed a protocol for older people with posttraumatic stress disorder (PTSD), subthreshold PTSD, or fear of falling resulting from a traumatic fall. ${ }^{62}$ In this American study the participants in the control group receive twice weekly a relaxation training of 8 in-home sessions of 75 minutes each; the participants in the intervention group receive twice weekly an exposure-based cognitive behavioral therapy of 8 inhome sessions of 75 minutes each. Results from both studies are expected soon.

Last, AMB-Home can be further refined by selecting the most effective components, as mentioned before. In addition, there are opportunities by using modern techniques, like websites or iPads ${ }^{63}$. This can make the program less labor intensive for health care professionals and therefore less costly. The same holds for translating the program into a volunteer lay leader model, which was already successful done for the group program. ${ }^{10}$ However, the feasibility and effectiveness of this new delivery methods have to be tested before large scale dissemination.

\section{Conclusion}

The home-based, cognitive behavioral program 'A Matter of Balance at Home' proved to be feasible as well as effective and cost-effective in reducing concerns about falls compared to usual care. After improvements like suggested in this chapter implementation in regular healthcare is recommended. Moreover, the program is expected to contribute to independent living of older people and provides an alternative for those who are not able or willing to attend group programs. 


\section{References}

1. Scheffer AC, Schuurmans MJ, van Dijk N, van der Hooft T, de Rooij SE: Fear of falling: measurement strategy, prevalence, risk factors and consequences among older persons. Age Ageing. 2008, 37(1):19-24.

2. Cumming RG, Salkeld G, Thomas M, Szonyi G: Prospective study of the impact of fear of falling on activities of daily living, SF-36 scores, and nursing home admission. J Gerontol A Biol Sci Med Sci. 2000, 55(5):M299-305.

3. Delbaere K, Crombez G, Vanderstraeten G, Willems T, Cambier D: Fear-related avoidance of activities, falls and physical frailty. A prospective community-based cohort study. Age Ageing. 2004, 33(4):368-373.

4. Denkinger MD, Lukas A, Nikolaus T, Hauer K: Factors associated with fear of falling and associated activity restriction in community-dwelling older adults: a systematic review. Am J Geriatr Psychiatry. 2015, 23(1):72-86.

5. Li F, Fisher KJ, Harmer P, McAuley E, Wilson NL: Fear of falling in elderly persons: association with falls, functional ability, and quality of life. J Gerontol B Psychol Sci Soc Sci. 2003, 58(5):P283-290.

6. Zijlstra GA, van Haastregt JC, van Eijk JT, van Rossum E, Stalenhoef PA, Kempen GI: Prevalence and correlates of fear of falling, and associated avoidance of activity in the general population of communityliving older people. Age Ageing. 2007, 36(3):304-309.

7. Zijlstra GA, van Haastregt JC, van Rossum E, van Eijk JT, Yardley L, Kempen GI: Interventions to reduce fear of falling in community-living older people: a systematic review. J Am Geriatr Soc. 2007, 55(4):603-615.

8. Bula CJ, Monod S, Hoskovec C, Rochat S: Interventions aiming at balance confidence improvement in older adults: an updated review. Gerontology. 2011, 57(3):276-286.

9. Kendrick D, Kumar A, Carpenter H, Zijlstra GAR, Skelton DA, Cook JR, Stevens Z, Belcher CM, Haworth D, Gawler SJ et al: Exercise for reducing fear of falling in older people living in the community. Cochrane $D b$ Syst Rev. 2014, 11.

10. Healy TC, Peng C, Haynes MS, McMahon EM, Botler JL, Gross L: The feasibility and effectiveness of translating a matter of balance into a volunteer lay leader model. J App/ Gerontol. 2008, 27(1):34-51.

11. Tennstedt S, Howland J, Lachman M, Peterson E, Kasten L, Jette A: A randomized, controlled trial of a group intervention to reduce fear of falling and associated activity restriction in older adults. J Gerontol B Psychol Sci Soc Sci. 1998, 53(6):P384-392.

12. Zijlstra GA, van Haastregt JC, Ambergen T, van Rossum E, van Eijk JT, Tennstedt SL, Kempen GI: Effects of a multicomponent cognitive behavioral group intervention on fear of falling and activity avoidance in community-dwelling older adults: results of a randomized controlled trial. J Am Geriatr Soc. 2009, 57(11):2020-2028.

13. Zijlstra GA, van Haastregt JC, Du Moulin MF, de Jonge MC, van der Poel A, Kempen GI: Effects of the implementation of an evidence-based program to manage concerns about falls in older adults. Gerontologist. 2013, 53(5):839-849.

14. Zijlstra GA, van Haastregt JC, van Eijk JT, de Witte LP, Ambergen T, Kempen GI: Mediating effects of psychosocial factors on concerns about falling and daily activity in a multicomponent cognitive behavioral group intervention. Aging Ment Health. 2011, 15(1):68-77.

15. Vlaeyen JWS, de Jong J, Geilen M, Heuts PHTG, van Breukelen G: The treatment of fear of movement/(re)injury in chronic low back pain: further evidence on the effectiveness of exposure in vivo. Clin J Pain. 2002, 18(4):251-261.

16. Resnicow K, Dilorio C, Soet JE, Borrelli B, Hecht J, Ernst D: Motivational interviewing in health promotion: it sounds like something is changing. Health Psychol. 2002, 21(5):444-451.

17. Cook TD, Campbell DT: Quasi-experimentation : design \& analysis issues for field settings. Chicago: Rand McNally College Pub. Co.; 1979.

18. Metzelthin SF, van Rossum E, de Witte LP, Ambergen AW, Hobma SO, Sipers W, Kempen GI: Effectiveness of interdisciplinary primary care approach to reduce disability in community dwelling frail older people: cluster randomised controlled trial. BMJ. 2013, 347:f5264.

19. Nyman SR, Victor CR: Older people's participation in and engagement with falls prevention interventions in community settings: an augment to the Cochrane systematic review. Age Ageing. 2012, 41(1):16-23. 
20. Delbaere K, Close JC, Mikolaizak AS, Sachdev PS, Brodaty H, Lord SR: The falls efficacy scale international (FES-I). A comprehensive longitudinal validation study. Age Ageing. 2010, 39(2):210-216.

21. Baranowski T, Stables G: Process evaluations of the 5-a-Day projects. Health Educ Behav. 2000, 27(2):157-166.

22. Linnan L, Steckler A: Process evaluation for public health interventions and research. An Overview. In: Process evaluation for public health interventions and research. Edited by Steckler A, Linnan L. San Francisco, CA: Jossey-Bass; 2002.

23. Bennett GA, Moore J, Vaughan T, Rouse L, Gibbins JA, Thomas P, James K, Gower P: Strengthening motivational interviewing skills following initial training: a randomised trial of workplace-based reflective practice. Addict Behav. 2007, 32(12):2963-2975.

24. Noordman J, van der Weijden T, van Dulmen S: Effects of video-feedback on the communication, clinical competence and motivational interviewing skills of practice nurses: a pre-test posttest control group study. J Adv Nurs. 2014, 70(10):2272-2283.

25. Pfister-Minogue KA, Salveson C: Training and experience of public health nurses in using behavior change counseling. Public Health Nurs. 2010, 27(6):544-551.

26. Daniels R: Frail elderly identification and disability prevention in primary care [PhD thesis]. Maastricht: Faculty of Health, Medicine and Life Sciences, University Maastricht; 2011.

27. Delbaere K, Close JC, Taylor M, Wesson J, Lord SR: Validation of the Iconographical Falls Efficacy Scale in Cognitively Impaired Older People. J Gerontol A Biol Sci Med Sci. 2013.

28. Yardley L, Beyer N, Hauer K, Kempen G, Piot-Ziegler C, Todd C: Development and initial validation of the Falls Efficacy Scale-International (FES-I). Age Ageing. 2005, 34(6):614-619.

29. Hadjistavropoulos T, Delbaere K, Fitzgerald TD: Reconceptualizing the role of fear of falling and balance confidence in fall risk. J Aging Health. 2011, 23(1):3-23.

30. Kowalski K, Rhodes R, Naylor PJ, Tuokko H, MacDonald S: Direct and indirect measurement of physical activity in older adults: a systematic review of the literature. Int J Behav Nutr Phys Act. 2012, 9:148.

31. Tinetti ME, Richman D, Powell L: Falls efficacy as a measure of fear of falling. J Gerontol. 1990, 45(6):P239-P243.

32. Sibley KM, Howe T, Lamb SE, Lord SR, Maki BE, Rose DJ, Scott V, Stathokostas L, Straus SE, Jaglal SB: Recommendations for a core outcome set for measuring standing balance in adult populations: a consensus-based approach. PLoS One. 2015, 10(3):e0120568.

33. Becker H, Stuifbergen A, Rogers S, Timmerman G: Goal attainment scaling to measure individual change in intervention studies. Nurs Res. 2000, 49(3):176-180.

34. Tennant A: Goal attainment scaling: Current methodological challenges. Disabil Rehabil. 2007, 29(2021):1583-1588.

35. Law M, Baptiste S, McColl M, Opzoomer A, Polatajko H, Pollock N: The Canadian occupational performance measure: an outcome measure for occupational therapy. Can J Occup Ther. 1990, 57(2):82-87.

36. Lachman ME, Agrigoroaei S: Promoting Functional Health in Midlife and Old Age: Long-Term Protective Effects of Control Beliefs, Social Support, and Physical Exercise. PLoS One. 2010, 5(10):-

37. Lachman ME, Jette A, Tennstedt S, Howland J, Harris BA, Peterson E: A cognitive-behavioural model for promoting regular physical activity in older adults. Psychol Health Med. 1997, 2(3):251-261.

38. Constantino MJ, Arnkoff DB, Glass CR, Ametrano RM, Smith JZ: Expectations. J Clin Psychol. 2011, 67(2):184-192.

39. Fritz CO, Morris PE, Richler JJ: Effect size estimates: current use, calculations, and interpretation. Journal of Experimental Psychology-General. 2012, 141(1):2-18.

40. Evans C, Margison F, Barkham M: The contribution of reliable and clinically significant change methods to evidence-based mental health. Evidence Based Mental Health. 1998, 1(3):70-72.

41. Jacobson NS, Truax P: Clinical significance: a statistical approach to defining meaningful change in psychotherapy research. J Consult Clin Psychol. 1991, 59(1):12-19.

42. Deshpande N, Metter EJ, Lauretani F, Bandinelli S, Ferrucci L: Interpreting fear of falling in the elderly: what do we need to consider? J Geriatr Phys Ther. 2009, 32(3):91-96. 
43. Vestjens L, Kempen GI, Crutzen R, Kok G, Zijlstra GA: Promising behavior change techniques in a multicomponent intervention to reduce concerns about falls in old age: a Delphi study. Health Educ Res. 2015, 30(2):309-322.

44. Stevens A, Beurskens A, Koke A, van der Weijden T: The use of patient-specific measurement instruments in the process of goal-setting: a systematic review of available instruments and their feasibility. Clin Rehabil. 2013, 27(11):1005-1019.

45. Strecher VJ, Seijts GH, Kok GJ, Latham GP, Glasgow R, DeVellis B, Meertens RM, Bulger DW: Goal setting as a strategy for health behavior change. Health Educ Q. 1995, 22(2):190-200.

46. Onghena P, Edgington ES: Customization of pain treatments: single-case design and analysis. Clin J Pain. 2005, 21(1):56-68; discussion 69-72.

47. Collins LM, Murphy SA, Strecher V: The multiphase optimization strategy (MOST) and the sequential multiple assignment randomized trial (SMART): new methods for more potent eHealth interventions. Am J Prev Med. 2007, 32(5 Suppl):S112-118.

48. Noonan RK, Sleet DA, Stevens JA: Closing the gap: a research agenda to accelerate the adoption and effective use of proven older adult fall prevention strategies. J Saf Res. 2011, 42(6):427-430.

49. Kilbourne AM, Neumann MS, Pincus HA, Bauer MS, Stall R: Implementing evidence-based interventions in health care: application of the replicating effective programs framework. Implement Sci. 2007, 2:42.

50. Simek EM, McPhate L, Hill KD, Finch CF, Day L, Haines TP: What are the characteristics of home exercise programs that older adults prefer?: A cross-sectional study. Am J Phys Med Rehabil. 2015, 94(7):508-521.

51. Prochaska JO, Diclemente CC: Trans-theoretical therapy - toward a more integrative model of change. Psychotherapy-Theory Research and Practice. 1982, 19(3):276-288.

52. Brug J, Glanz K, Kok G: The relationship between self-efficacy, attitudes, intake compared to others, consumption, and stages of change related to fruit and vegetables. Am J Health Promot. 1997, 12(1):25-30.

53. Raad voor de Volksgezondheid \& Zorg: Perspectief op gezondheid 20/20. Den Haag: RVZ; 2010.

54. Huber M, Knottnerus JA, Green L, Horst Hvd, Jadad AR, Kromhout D, Leonard B, Lorig K, Loureiro MI, Meer JWMvd et al: How should we define health? BMJ. 2011, 343.

55. Powell LE, Myers AM: The activities-specific balance confidence (ABC) scale. J Gerontol A Biol Sci Med Sci. 1995, 50A(1):M28-34.

56. Yardley L, Smith H: A prospective study of the relationship between feared consequences of falling and avoidance of activity in community-living older people. Gerontologist. 2002, 42(1):17-23.

57. Jorstad EC, Hauer K, Becker C, Lamb SE, ProFa NEG: Measuring the psychological outcomes of falling: a systematic review. J Am Geriatr Soc. 2005, 53(3):501-510.

58. Moore DS, Ellis R: Measurement of fall-related psychological constructs among independent-living older adults: a review of the research literature. Aging Ment Health. 2008, 12(6):684-699.

59. Brouwer B, Musselman K, Culham E: Physical function and health status among seniors with and without a fear of falling. Gerontology. 2004, 50(3):135-141.

60. Hadjistavropoulos T, Martin RR, Sharpe D, Lints AC, McCreary DR, Asmundson GJ: A longitudinal investigation of fear of falling, fear of pain, and activity avoidance in community-dwelling older adults. $J$ Aging Health. 2007, 19(6):965-984.

61. Parry SW, Deary V, Finch T, Bamford C, Sabin N, McMeekin P, O'Brien J, Caldwell A, Steen N, Whitney SL et al: The STRIDE (strategies to increase confidence, independence and energy) study: cognitive behavioural therapy-based intervention to reduce fear of falling in older fallers living in the community study protocol for a randomised controlled trial. Trials. 2014, 15(1):210.

62. Jayasinghe N, Sparks MA, Kato K, Wilbur K, Ganz SB, Chiaramonte GR, Stevens BL, Barie PS, Lachs MS, O'Dell $\mathrm{M}$ et al: Exposure-based CBT for older adults after fall injury: description of a manualized, timelimited intervention for anxiety. Cogn Behav Pract. 2014, 21(4):432-445.

63. Delbaere K, Valenzuela T, Woodbury A, Davies T, Yeong J, Steffens D, Miles L, Pickett L, Zijlstra GAR, Clemson $L$ et al: Evaluating the effectiveness of a home-based exercise programme delivered through a tablet computer for preventing falls in older community-dwelling people over 2 years: study protocol for the Standing Tall randomised controlled trial. BMJ Open. 2015, 5(10). 


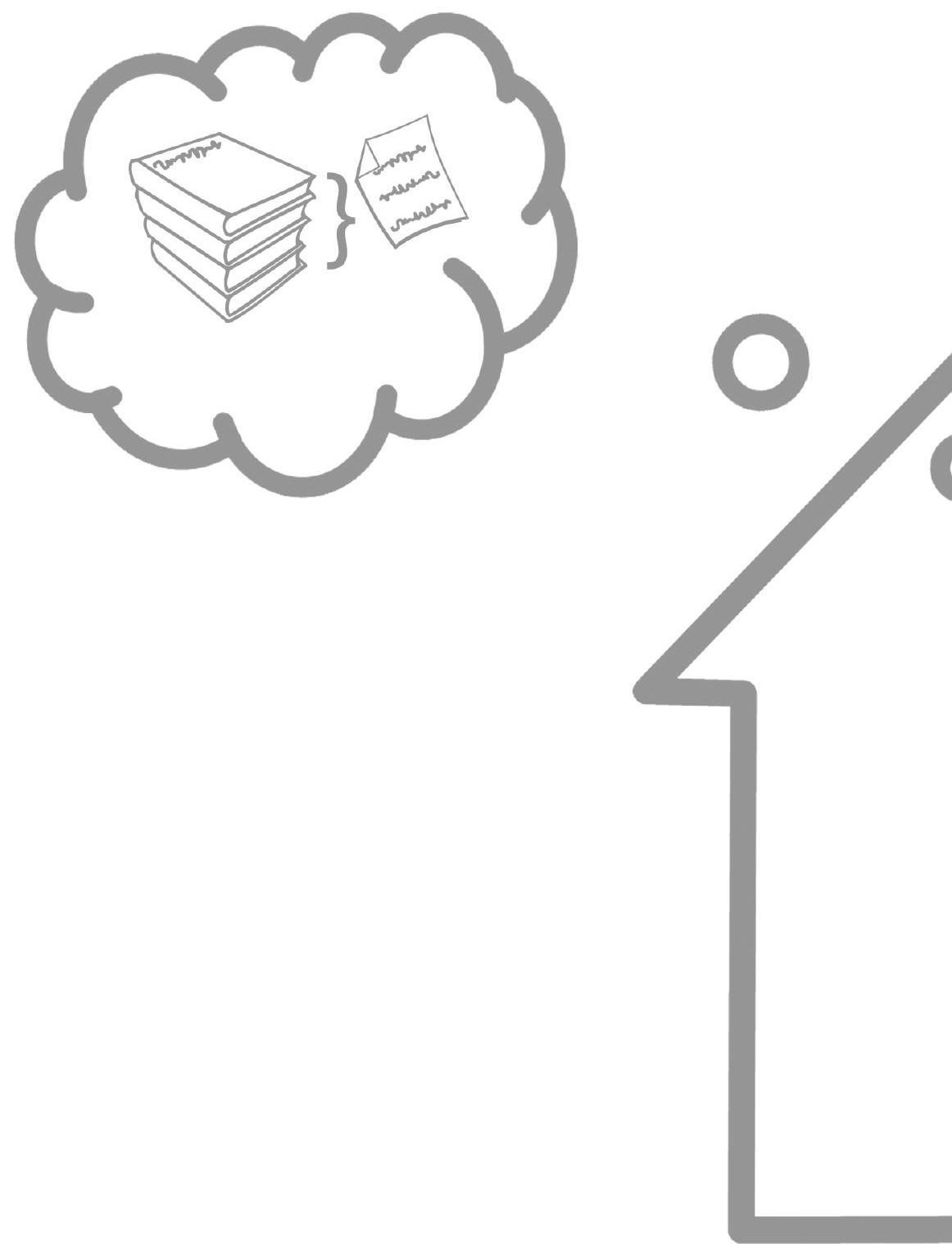



\section{Summary}

Concerns about fall are a common health problem and a threat to autonomy for community-dwelling older people. These concerns are associated with adverse outcomes in psychosocial, physical and functional domains such as falls, avoidance of activity, decreased balance performance, decreased mobility, lower social participation, functional decline, low quality of life, and loss of independence. In addition to the negative consequences of concerns about falls, there is the risk of increased healthcare usage and related costs. Reviews showed that a few approaches are successful in reducing concerns about falls and potentially contributors to a better quality of life and independent living of older people. The multicomponent, cognitive behavioral group program 'A Matter of Balance' (AMB) is one of the these few interventions that targets psychosocial, physical and functional aspects of concerns about falls and consistently shows positive effects on a broad range of outcomes. Despite its success in (cost-)effectiveness, it seems that a substantial number of eligible participants do not attend or complete the group program because of health problems. To enable these frail, community-dwelling older people to participate, a tailor-made, home-based format, named 'A Matter of Balance at Home' (AMB-Home) was developed and evaluated.

Chapter 1 gives an overview of the definitions of concerns about falls, theoretical models, prevalence, risks factors, consequences and developed programs. Furthermore, the three main objectives of this thesis are described, namely: 1) explore which format(s) are preferred to manage concerns about falls by community-dwelling older people; 2) study the feasibility and acceptability of a home-based, cognitive behavioral program to manage concerns about falls in frail, community-dwelling, older people; and 3) analyze whether the newly developed program is (cost-)effective with respect to the reduction of concerns about falls when compared to care as usual.

In Chapter 2 the results of a cross-sectional study among 5,755 communitydwelling older people are described. In this study the preferences regarding six different formats (in a group, at home, via telephone, via a combination of home visits and telephone consultations, via television or via internet) of a program that primarily focuses on managing concerns about falls were explored. Of the 2,498 responders, $62.7 \%$ indicated having no interest in any of the formats. The willingness to participate varied per program format and ranged from $21.5 \%$ (at home) to $9.4 \%$ (via internet). Higher levels of fall-related concerns were associated with increased preference for a program including home visits. Poor perceived health and older age ( $\geq 80$ years) were associated with less preference for a group program.

Chapter 3 describes the development of a home-based, cognitive behavioral program to manage concerns about falls, named 'A Matter of Balance at Home' (AMBHome) and the design of a process, effect, and economic evaluation study. A group program of eight sessions was transformed to an individual format of seven sessions 
(three home-visits and four telephone contacts). The sessions were aimed at instilling adaptive and realistic views about falls, as well as increasing activity and safe behavior. Overall, principles for behavior change and themes of the group program were maintained in AMB-Home. However, in adapting the group program to a home-based program, several adaptations were implemented. For instance, the physical exercises in the group program were replaced by 'exposure in vivo', motivational interviewing was incorporated to encourage internal motivation to change behavior and increase selfefficacy, and participants were encouraged to invite a significant other (e.g., a spouse, friend, or neighbor) to be present during the home visits. AMB-Home was facilitated by trained community nurses who were qualified in the field of geriatrics and worked at local home-care agencies. The program was evaluated in a randomized controlled trial. Screening for eligibility was done with a short postal questionnaire in a general older population living in the southeast of the Netherlands. Older people were included when they were 70 years of age and older; living in the community; reported at least some concerns about falls and at least some associated avoidance of activity and when they perceived their general health as fair or poor. For the effect evaluation participants received a baseline measurement and a 5-month and 12-month follow-up measurement. The primary outcome of the effect evaluation was concerns about falls. The process evaluation measured: the characteristics of the population that was reached; protocol adherence by facilitators; protocol adherence by participants (engagement in exposure and homework); opinions about the program of participants and facilitators; perceived benefits and achievements; and experienced barriers. The economic evaluation examined the impact on health-care utilization, as well as related costs.

Chapter 4 presents the findings of the process evaluation to get a better understanding of the feasibility and acceptability of the AMB-Home program. The following elements were measured: participation of the target population in the program (reach); implementation of the program as planned (fidelity); participants' engagement in and receptivity to the program (dose received - exposure); participants' and facilitators' satisfaction with the program (dose received - satisfaction); and aspects of the program that need improvement prior to nationwide implementation (barriers). Data were collected from eight nurses (the facilitators of the program) and 194 program participants. Of the 194 participants, 117 (60\%) completed the program (i.e., received at least five of the seven sessions). The main reason of participants for not completing was lost interest. According to the self-reports of facilitators the program was generally performed as planned. However, outcomes of audio recordings were inconsistent with these selfreports and showed that the performance according to protocol was much lower. A large majority of participants indicated that they had reached at least one personal goal and that they experienced benefits from the program in daily life. Also the performance of the facilitator was highly valued by the participants. Facilitators indicated as a barrier that more than one third of the 117 participants who completed the program should not have enrolled in the program as they considered them not the target sample, for 
example because of physical limitations or not being restricted in daily activities by concerns to fall.

In Chapter 5 the results of the effect evaluation are presented. In total, 398 participants were included in the study. The mean age was 78.3 years and of those included $70 \%$ was female. Through randomization, 195 participants were allocated to the control group, and 194 participants were included in the intervention group. Data were collected from 172 control and 139 intervention participants directly after the intervention and from 162 control and 133 intervention participants at 12 months follow-up. Main reasons for lost follow-up were similar in both groups, namely health problems and lost interest. Mixed-effects linear regression analyses showed significant lower levels of concerns about falls in the intervention group at 12 months compared with the control group. Furthermore, significant reductions in self-reported activity avoidance, restrictions in daily life and indoor falls were identified in the intervention group compared to the control group. Effect sizes were small to medium. No significant difference in total number of falls was noted between the groups.

Chapter 6 reports on the cost-effectiveness of the program. The economic evaluation was embedded in the trial and performed from a societal perspective with a time horizon of 12 months. The main outcome measure for the cost-effectiveness (CEA) was concerns about falls and the outcome for the cost-utility analysis (CUA) was Quality Adjusted Life Years (QALY). The total societal costs for the usual care group is 8,094 Euro per person and for the intervention group 7,890 Euro per person including 716 Euro program costs per participant. Total costs and subcategories of costs were comparable between the two groups. After synthesizing the effects and costs, the probability that the program was cost-effective for concerns about falls was substantially as well as for QALYS as outcome. Additional sensitivity analyses showed that if participants received 5 or more sessions the probability that the program was cost-effective increased. When costs were taken from a healthcare perspective the probability of the costeffectiveness of the program decreased.

In Chapter 7 the main results of this thesis are presented and discussed. Moreover, recommendations for clinical practice, implementation, and for future research are given. Altogether, the home-based, cognitive behavioral program 'A Matter of Balance at Home' proved to be feasible as well as effective and cost-effective in reducing concerns about falls compared to usual care. Therefore, implementation in regular Dutch healthcare is recommended after some minor improvements. 

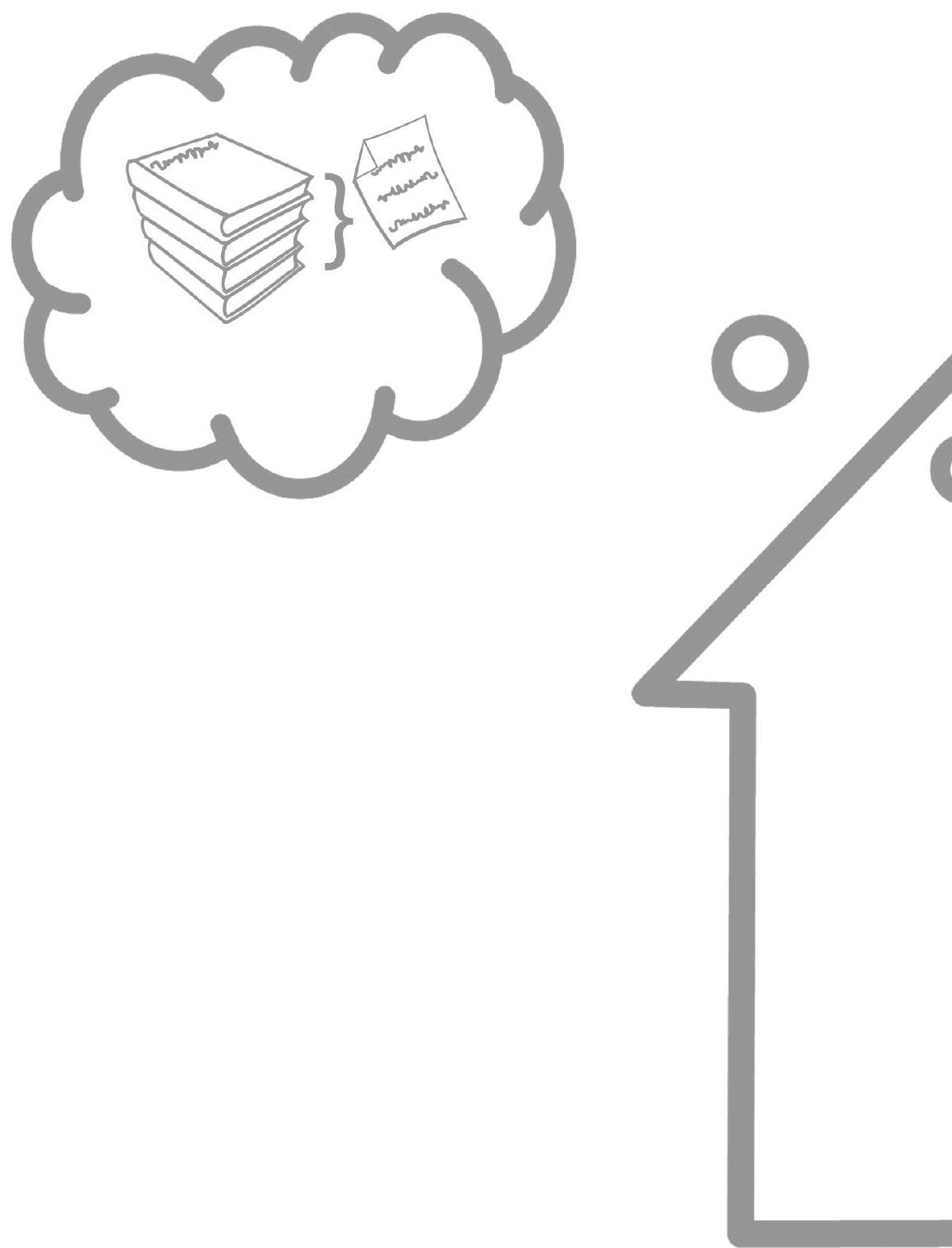
Samenvatting 



\section{Samenvatting}

Bezorgdheid om te vallen is een algemeen probleem onder zelfstandig wonende ouderen en vormt een bedreiging voor hun zelfredzaamheid. Deze bezorgdheid is geassocieerd met negatieve gevolgen in het psychosociale, fysieke en functionele domein, zoals vallen, vermijding van activiteiten, verminderde balans, verminderde mobiliteit, lagere maatschappelijke participatie, functionele achteruitgang, lage kwaliteit van leven en verlies van zelfstandigheid. Naast deze negatieve gevolgen van bezorgdheid om te vallen, is er ook een risico op hoger zorggebruik en daaraan gerelateerde kosten. Overzichtsartikelen laten zien dat enkele benaderingen succesvol zijn in het reduceren van bezorgdheid om te vallen en daarmee in potentie bijdragen aan een betere kwaliteit van leven en zelfstandigheid van ouderen. De uit meerdere componenten bestaande, cognitief-gedragsmatige groepscursus 'Zicht op Evenwicht' (eerder ook aangeduid als 'Beter in Balans' en oorspronkelijk genaamd 'a Matter of Balance') is een van de weinige interventies die gericht is op psychosociale, fysieke en functionele aspecten van bezorgdheid om te vallen en consistent positieve effecten laat zien op een brede waaier van uitkomsten. Ondanks het succes in (kosten)effectiviteit is ook gebleken dat een aanzienlijk deel van potentieel geschikte deelnemers niet naar de groepscursus komt of deze niet afmaakt vanwege gezondheidsproblemen. Om ook deze kwetsbare groep van zelfstandig wonende ouderen te bereiken is een individuele thuisversie ontwikkeld en geëvalueerd onder de naam 'Thuis in Balans'.

Hoofdstuk 1 geeft een overzicht van de definities van bezorgdheid om te vallen, theoretische modellen, prevalentie, risicofactoren, consequenties en ontwikkelde programma's. Daarnaast worden de drie hoofddoelen van het proefschrift beschreven, namelijk: 1) verkenning van voorkeuren van zelfstandig wonende ouderen wat betreft verschillende vormen van een cursus om bezorgdheid om te vallen te verminderen; 2) evaluatie van uitvoerbaarheid en acceptatie van een cognitief-gedragsmatige cursus aan huis om bezorgdheid om te vallen te verminderen bij zelfstandig wonende ouderen; 3) evaluatie van effecten van de nieuw ontwikkelde cursus op bezorgdheid om te vallen, zorggebruik en kosten in vergelijking met de reguliere zorg.

In Hoofdstuk 2 worden de resultaten van een cross-sectionele studie onder 5.755 zelfstandig wonende ouderen weergegeven. In deze studie werden de voorkeuren onderzocht ten opzichte van zes verschillende cursusvormen (in een groep, via de telefoon, thuis, een combinatie van huisbezoeken en telefonische contacten, via de televisie of via internet) die allen gericht zijn op het verminderen van bezorgdheid om te vallen. Van de 2.498 ouderen die op de vragenlijst reageerden, gaf 62,7\% aan überhaupt geen interesse te hebben in een cursus. De bereidheid om mee te doen varieerde per cursusvorm van $21,5 \%$ (thuis) tot $9,4 \%$ (via internet). Meer bezorgdheid om te vallen was geassocieerd met een grotere voorkeur voor een cursus waarbij huisbezoeken een onderdeel vormden. Een slechtere ervaren gezondheid en een hogere leeftijd ( $\geq 80$ jaar) bleken geassocieerd te zijn met een kleinere voorkeur voor een groepscursus. 
Hoofdstuk 3 geeft een beschrijving van de ontwikkeling van een cognitiefgedragsmatige cursus aan huis om bezorgdheid om te vallen te verminderen, genaamd 'Thuis in Balans' (TIB), en de opzet van een proces-, effect- en economische evaluatie. Een groepscursus van acht bijeenkomsten werd omgevormd tot een individuele vorm met zeven contactmomenten: drie huisbezoeken en vier telefonische contacten. De contacten waren gericht op het ontwikkelen van adaptieve en realistische opvattingen over vallen, het verhogen van de dagelijkse activiteit en veilig gedrag. In het algemeen werden de principes van gedragsverandering en thema's van de groepscursus gehandhaafd in de thuisversie. Desalniettemin werden er een aantal aanpassingen gedaan bij het omzetten van de groepscursus naar de individuele variant. Zo werden de lichamelijke oefeningen uit de groepscursus vervangen door een 'exposure in vivo' experiment, werd motiverende gesprekstechniek toegevoegd om interne motivatie tot gedragsverandering te stimuleren net als het vergroten van het zelfvertrouwen en werden deelnemers gestimuleerd om een bekende uit te nodigen om de huisbezoeken bij te wonen (bijv. een partner, vriend(in), of buurvrouw of -man) voor sociale steun. TIB werd uitgevoerd door een getrainde wijkverpleegkundige van de thuiszorg met een specialisatie in geriatrische zorg. De cursus werd geëvalueerd in een gerandomiseerde gecontroleerde studie. Het selecteren van geschikte kandidaten vond plaats middels een korte schriftelijke vragenlijst verspreid onder de algemene zelfstandig wonende oudere populatie in Zuid-Limburg. Ouderen werden geïncludeerd als zij 70 jaar of ouder waren, tenminste soms bezorgd waren om te vallen en tenminste soms activiteiten vermeden vanwege deze bezorgdheid en als zij hun gezondheid als matig of slecht ervaarden. Voor de effectevaluatie ontvingen deelnemers een basismeting en een nameting na zeven maanden en na twaalf maanden. De hoofduitkomstmaat van de effectevaluatie was bezorgdheid om te vallen. In de procesevaluatie werden de volgende zaken gemeten: de karakteristieken van de groep die bereikt is; in hoeverre de cursus is uitgevoerd volgens protocol; in hoeverre deelnemers actief hebben deelgenomen aan de cursus; mening van cursusbegeleiders en deelnemers over de cursus; ervaren baten; en waargenomen barrières. In de economische evaluatie werd gekeken naar de effecten van de cursus op zorggebruik en gerelateerde kosten.

Hoofdstuk 4 presenteert de bevindingen van de procesevaluatie die meer inzicht geven in de uitvoerbaarheid en acceptatie van de cursus. De volgende elementen werden daar in meegenomen: deelname van de doelgroep aan de cursus (bereik); implementatie van de cursus volgens plan (therapietrouw); betrokkenheid bij de cursus en ontvankelijkheid van de deelnemers (ontvangen dosis - blootgestaan); tevredenheid met de cursus van zowel deelnemers als cursusbegeleiders (ontvangen dosis - tevredenheid); en aspecten van de cursus die verbeterd dienen te worden voordat overgegaan kan worden tot verdere implementatie (barrières). De gegevens werden verzameld bij acht verpleegkundigen (begeleiders van de cursus) en 194 cursusdeelnemers. Van de 194 deelnemers maakten 117 deelnemers (60\%) de cursus af (d.w.z. vijf of meer van de zeven contacten). De belangrijkste reden om vroegtijdig te stoppen was 'geen 
interesse meer om de cursus te starten of af te ronden. Op basis van de zelfrapportages van de cursusbegeleiders blijkt dat de cursus voor het grootste deel volgens plan is uitgevoerd. Daartegenover bleek uit de resultaten van geluidsopnamen van contactmomenten dat het cursusprotocol beduidend minder goed was gevolgd. Een grote meerderheid van de deelnemers gaf aan dat zij tenminste één van de vooral gestelde doelen hadden bereikt en dat zij baat ervaarden van de cursus in het dagelijks leven. Ook het optreden van de cursusbegeleider werd door de deelnemers zeer gewaardeerd. Cursusbegeleiders rapporteerden als barrière dat de cursus niet geschikt was voor alle geïncludeerde deelnemers. In hun ogen behoorde meer dan een derde van de 117 deelnemers niet tot de doelgroep, bijvoorbeeld omdat zij fysiek beperkt waren of niet beperkt in hun dagelijkse activiteiten door bezorgdheid om te vallen.

In hoofdstuk 5 zijn de resultaten beschreven van de effectevaluatie. In totaal werden 398 deelnemers geïncludeerd in de studie. De gemiddelde leeftijd was 78,3 jaar en $70 \%$ van de deelnemers was vrouw. Door middel van randomisatie werden 195 deelnemers toegewezen aan de controle groep en 194 aan de interventie groep. Bij 172 en 139 deelnemers van respectievelijk de controle en interventie groep werden direct na de interventie gegevens verzameld. Twaalf maanden na de eerste meting bedroeg dit 162 deelnemers van de controle groep en 133 deelnemers van de interventie groep. De belangrijkste redenen voor uitval waren in beide groepen vergelijkbaar, namelijk gezondheidsproblemen en verloren interesse. Uit de mixed-effecten lineaire regressie analyses bleek dat na 12 maanden de bezorgdheid om te vallen significant minder was in de interventie groep ten opzichte van de controle groep. Daarnaast rapporteerden deelnemers in de interventie groep significant minder vermijding van activiteiten vanwege bezorgdheid om te vallen, minder beperkingen in het dagelijks leven en minder valincidenten binnenshuis in vergelijking met deelnemers in de controle groep. Effectgroottes waren klein tot middelgroot. Er was geen significant verschil in totaal aantal valincidenten tussen beiden groepen.

Hoofdstuk 6 rapporteert over de kosten en baten van de cursus. De economische evaluatie was integraal onderdeel van de studie en is uitgevoerd vanuit een maatschappelijk perspectief met een tijdshorizon van 12 maanden. De hoofduitkomst van de kosten-effectiviteitsanalyse was bezorgdheid om te vallen en de uitkomst van de kostenutiliteitsanalyse was gewonnen levensjaren in goede gezondheid. In totaal waren de gemiddelde (maatschappelijke) kosten gedurende de studieperiode voor de controle groep 8.094 Euro per persoon en voor de interventie groep 7.890 Euro per persoon, inclusief 716 Euro per deelnemer voor de cursus. Na het samenvoegen van de effecten en de kosten was de kans aanzienlijk dat de cursus kosteneffectief is, dat geldt zowel voor bezorgdheid om te vallen als voor de uitkomst gewonnen levensjaren in goede gezondheid. Aanvullende sensitiviteitsanalyses lieten zien dat als deelnemers aan vijf of meer contacten hadden deelgenomen, de kans dat de cursus kosteneffectief is toeneemt. Wanneer de kosten vanuit het perspectief van de gezondheidszorg worden genomen, daalt de kans dat de cursus kosteneffectief is. 


\section{SAMENVATTING}

In hoofdstuk 7 worden de belangrijkste resultaten samengevat en bediscussieerd. Vervolgens worden er aanbevelingen gedaan voor zowel de praktijk, implementatie als voor toekomstig onderzoek. Alles samengenomen heeft de cognitief-gedragsmatige cursus aan huis 'Thuis in Balans' laten zien dat zij zowel uitvoerbaar, effectief en kosteneffectief is in vergelijking met reguliere zorg. Daarom wordt, na een aantal kleine verbeteringen, implementatie van de cursus in de reguliere Nederlandse zorg aanbevolen. 


Valorization 



\section{Valorization}

This section focuses on how the results of this thesis can be used by scientists as well as by others, like healthcare professionals, policy makers, health insurance companies and older people.

\section{Relevance}

One of the most common fear among older people is the fear of falling. This particular fear associated with concerns about potential falls, has great impact on older people's daily life and may be a threat to independent living of those who it affects. At the same time, national policies worldwide encourage and facilitate older people to live independently as long as possible. This policy reflects the preference of many older people themselves and is in line with the need to keep the healthcare costs affordable with the demographic trend of an increasing proportion of people aged 65 years and over. Therefore, interventions that support older people in their independence are needed. Self-management skills, like problem solving, resource utilization and action planning, can be a powerful tool in order to achieve this goal by community-dwelling older people.

\section{Target groups}

The home-based, cognitive behavioral program 'A Matter of Balance at Home' (AMBHome) stimulates the self-management skills of frail community-living older people. The program proved to be feasible as well as effective and cost-effective in reducing concerns about falls compared to usual care. Moreover, the program contributes to improved daily functioning in older people and consequently, implementation in regular healthcare is recommended. To put a widespread dissemination and implementation of this evidence based program into practice different target groups are identified. First, (home care) health organizations need to be reached and convinced about the added value of offering the program to their clients. This could be achieved by informing their trade and/or professional associations. Also the Centre for Healthy Living ('Centrum Gezond Leven') of the National Institute for Public Health and the Environment (RIVM) may facilitate further implementation. The program is included in their intervention database and this database is being consulted on regular basis by policymakers and practitioners (www.loketgezondleven.nl/-interventies/i-database/1400744). Furthermore, health insurance companies could stimulate the use of the program by reimbursing (part of) the costs of the program for their customers.

\section{Products}

AMB-Home is an individual, cognitive behavioral program to manage concerns about falls. The program comprises seven individual sessions, consisting of three home-visits of 60,60 and 75 minutes, respectively, and four telephone contacts of 35 minutes each. 
The program is founded on an evidence based group program and uses principles of cognitive restructuring, social modelling, education and other strategies for behavioral change. Because of the positive results, AMB-Home is made available by the National Institute of Mental Health and Addiction in the Netherlands (Trimbos institute) in cooperation with Maastricht University under the name of 'Zicht op Evenwicht'. Potential facilitators of the program, like community nurses, physiotherapists or occupational therapists, can attend an one-day training at the Trimbos Institute. In this training they get familiar with the main principles of the group as well as the individual format of the Dutch version of 'A Matter of Balance'. After this training the facilitators deliver the course independently, supported by the detailed handbook for facilitators and additional materials. In this way the program is hosted and delivering is guaranteed for the long term, see also www.zichtopevenwicht.nl.

\section{Health care professionals}

There is a paradigm shift in healthcare towards people who are more responsible for their own health and well-being. In this self-management paradigm health care professionals have to act as supporters of independence of older people. AMB-Home was way ahead of its time and meets this new model of health and healthcare. Consequently it is an added value for health care professionals. A few recommendations could be given to facilitators who will offer the program to older people. First, a face-to-face intake procedure could prevent dropout of participants during the program. Eligibility and motivation of potential participants for the program can be checked during this intake session, moreover, clear information about the program can be provided to establish matching and mutual expectancies. Second, motivational interviewing is used as a technique to encourage active participation and behavior change of the participant in the present study. From observations during the present study and based on results from previous studies we conclude that more training of the facilitators is needed to master this behavior change counseling skill. Motivational interviewing is, however, a widely applicable tool for health care professionals in daily practice (e.g. with respect to medication adherence). Therefore an additional general training in this matter could be justified. Lastly, to replace the social support from other participants, which is an important component in the group format of $A M B$, participants were encouraged to invite a significant other, for example, a spouse, other relative, friend, or neighbor. Facilitators were highly positive about the role of the significant other in situations where someone was present. Based on these experiences more efforts on inviting a significant other could be recommended.

\section{Policy makers and health insurance companies}

The responsibility of delivering many healthcare duties is currently shifted in the Netherlands from the national government to the local government and to healthcare agencies. This offers opportunities for more customized prevention. And as mentioned be- 
fore, health is no longer considered as a state of total well-being, but rather the ability to adapt and to self-manage in the face of social, physical, and emotional challenges (Huber et al., 2011). AMB-Home perfectly fits with these new developments and should therefore be adopted and financially supported by healthcare agencies and local governments.

\section{Researchers and future research}

During the last decades of research in the area of concerns about falling, different terms were introduced accompanied with different measures. The introduction of all these constructs of fall-related psychological concerns have led to confusion about which construct is meant in a study; instruments are often used to measure constructs other than for which the instruments were designed. Clear guidelines about which measure should be used to assess which construct of fall-related psychological concerns are needed. Moreover, consensus about a common outcome data set for the examination of fall-related psychological concerns and related factors would be a great step forward in the field of concerns about falls. In addition, much is known about the risk factors associated with fall-related psychological constructs, although much less is known about causal relationships between these risk factors and such outcomes. More knowledge about the offset and development of concerns about falls may help in establishing risk profiles and tailoring the programs to the needs of those who it regards. Furthermore, outcomes of comparable studies may support the external validity of our results and increase the evidence that an individual cognitive behavioral approach can contribute to the management of concerns about falls. Last, AMB-Home can be further refined and improved by testing and selecting the most effective components. There are also opportunities by using modern techniques, like websites or iPads. This can make the program less labor intensive for health care professionals and therefore less costly. The same holds for translating the program into a volunteer lay leader model, which is a very successful format for the group program in the US. However, the feasibility and effectiveness of this new delivery methods have to be examined before large scale dissemination. 

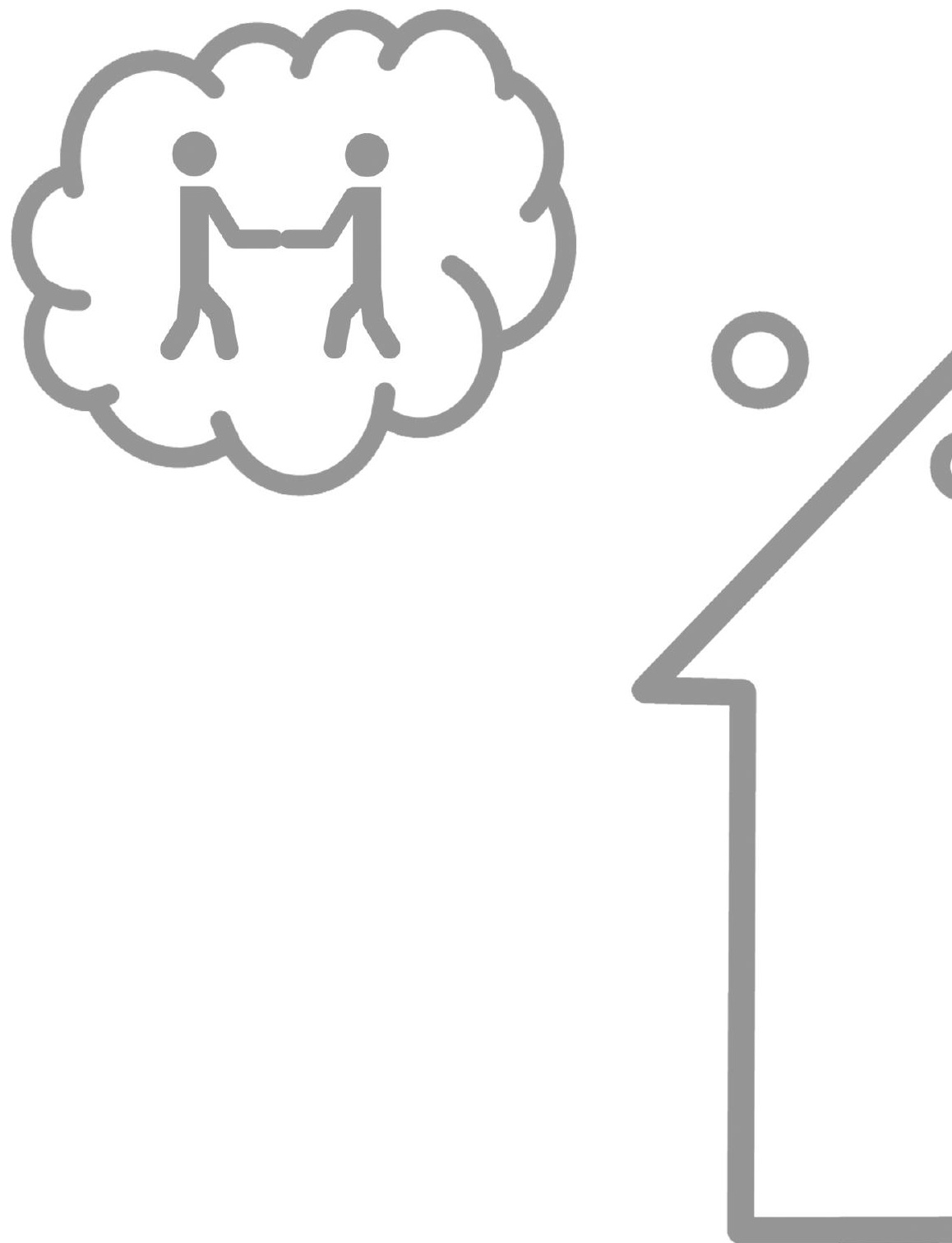
Dankwoord 



\section{Dankwoord}

De laatste loodjes wegen het zwaarst of beter gezegd duren het langst, maar het is gelukt. Mijn proefschrift is af en dat was zonder de hulp van velen in de afgelopen jaren niet gelukt. Een aantal daarvan wil ik graag noemen.

Als eerste wil ik graag alle ouderen die hebben deelgenomen aan het onderzoek en de verpleegkundigen die de cursus hebben uitgevoerd bedanken. Zonder jullie was het onderzoek niet mogelijk geweest. Daarbij wil ik ook de drie thuiszorgorganisaties in Zuid-Limburg noemen die hun medewerking aan het onderzoek hebben verleend, te weten 'Envida' (voorheen 'GroenekruisDomicura') in Maastricht, 'Zorggroep Meander' in Heerlen en 'Zuyderland' (voorheen 'Orbis Thuiszorg') in Sittard-Geleen.

Ruud Kempen, Johan Vlaeyen en Rixt Zijlstra, ik wil jullie bedanken voor de mogelijkheid die jullie mij gegeven hebben om dit promotieonderzoek uit te voeren. Ik denk dat jullie je weleens afgevraagd hebben of het ooit af zou komen, maar ik heb er altijd vertrouwen in gehad en jullie zijn mij, ondanks mogelijke twijfels, blijven begeleiden. En zie hier het resultaat. Ruud, jij bent altijd de rust zelve. Ik vond het heel erg prettig hoe snel en grondig je naar mijn stukken keek en ik vervolgens ook de ruimte ervaarde om daar zelf weer verder mee aan de gang te gaan. Rixt, vanaf het begin af aan ben jij altijd heel erg druk geweest, maar probeerde je er tegelijk zoveel mogelijk voor mij te zijn. Je was heel nauw betrokken bij de opzet en praktische invulling van het hele onderzoek. Jouw kritische blik heeft weleens verlammend op mij gewerkt, maar ik heb tegelijk ook ontzettend veel van je geleerd. Johan, jij stond (fysiek) wat verder af van het project, maar door jouw frisse blik en andere achtergrond heb je het onderzoek buitengewoon verrijkt. En ook mij heb je keer op keer gestimuleerd om met een bredere blik te kijken.

De projectgroep bestaande uit Geert Crombez, Erik van Rossum, Kim Delbaere, Jan Hamers en Jolanda van Haastregt, bedankt dat jullie je kennis en ervaring met mij wilde delen. Ondanks de afstand tussen Maastricht, Leuven en Sydney was de opkomst altijd hoog tijdens de overleggen.

Ton van Ambergen en Silvia Evers, jullie hebben mij enorm geholpen bij respectievelijk de effectanalyses en kostenanalyses. Bedankt hiervoor.

I would like to thank the members of the assessment committee, Prof. Gerjo Kok as chair, Prof. Susanne Iwarsson, Prof. Trudy van der Weijden, Prof. Carmen Dirksen and Prof. Frank Buntinx for reviewing my thesis. 
Yvonne, de eerste twee jaar waren wij de kartrekkers van het project. Je hebt me ontzettend geholpen bij de dataverzameling, zonder jouw inzet was het niet gegaan. Al botsten we af en toe over de aanpak van de werkzaamheden, onze gedeelde afkomst beiden waren we opgegroeid op een boerderij - zorgden voor een band. Ook de andere studentassistenten die later hebben meegeholpen met de dataverzameling wil ik graag bedanken. En MEMIC wil ik bedanken voor de fijne samenwerking in de personen van Anita Legtenberg en Alfons Schroten die waren betrokken bij het opzetten van het logistieke systeem en het interviewteam onder leiding van Marlène Ronner die zorgden voor de metingen.

Beste oud-collega's van HSR jullie hebben mijn tijd bij de universiteit een heel plezierige gemaakt. Het was heerlijk om af en toe afleiding te hebben bij het koffie halen of even binnen te kunnen lopen voor zowel werk als privé gerelateerde zaken. Esther, Silke, Jill, Bart, Noémie, Josianne, Basema en Donja met jullie heb ik een groot deel van mijn promotietraject gedeeld en daarmee kijk ook terug op vele leuke momenten tijdens congressen en uitjes. Inmiddels zijn jullie mij allen voorgegaan in het afronden, maar nu kunnen we dan ook eindelijk mijn feestje gaan vieren. Ruben, jij was mijn laatste kamergenoot. Jammer dat het maar voor zo'n korte periode was, want jouw enthousiasme werkte aanstekelijk.

Nieuwe collega's, en dan met name het team gezondheidsbevordering bij de GGD Limburg-Noord, onderdeel van de veiligheidsregio Limburg-Noord, jullie hebben het laatste staartje van het promoveren nog meegekregen. Jullie medeleven en de flexibele houding ervaar ik als heel prettig. Vanaf nu kan ik me weer gaan richten op één baan en dat maakt het wel zo overzichtelijk.

Lieve vriendinnetjes uit Werkhoven, Eline, José, Maaike, Marion en Naftali, we kennen elkaar al sinds de basisschool, dus inmiddels delen we al bijna dertig jaar lief en leed. Vakanties, weekenden, etentjes en stapavonden, zoveel goede herinneringen, ik hoop dat onze vriendschap nog heel lang zal duren en vanaf nu kan ik er ook weer wat vaker bij zijn. Marjolein, jij zat een klas hoger en hoort daarom niet bij het clubje hierboven, maar jouw lange vriendschap en belangstelling die je in de afgelopen jaren hebt getoond, zijn mij ook zeer dierbaar.

Hilde, Floortje, Theisje, Daan, Joost, Joris, Tim en Timme in 2001 vormde wij de Jaarclub Zuur. Ondanks de verspreiding over het land na ons afstuderen vinden we elkaar nog zeer regelmatig, bijvoorbeeld als er weer iets te vieren is, zo mogen we ons nu toch wel een zeergeleerde JC noemen met straks drie doctoren.

Naima, wat fijn dat jij mijn paranifm wil zijn. Wij leerde elkaar kennen in ons eerste studiejaar in Maastricht toen we allebei in Veldwezelt woonde. De frequentie dat we 
elkaar zien is afgenomen van bijna iedere dag naar een paar keer per jaar, maar als ik je spreek of zie is het even vertrouwd.

Gerda, jou moet ik ook zeker noemen, want zonder jouw liefdevolle zorg voor onze kinderen, had ik met heel wat meer schuldgevoel al die dagen aan mijn proefschrift gewerkt. Bedankt dat je er voor onze kinderen wilt zijn!

Rafiq, Tessel, Carlijn, Maaike en Bernard, jullie interesse in mijn onderzoek de afgelopen jaren tijdens de vele familiefeestjes of vakanties was erg fijn. Tegelijk was het ook prima om het er niet over te hebben en te genieten van de kinderen.

Jo en Hanneke, schoonouders heb je niet voor het uitkiezen, maar ik kan me geen betere voorstellen. Jopadag één keer per week voor de kinderen en inmiddels komt Hanneke vaak mee, maar ook alle fantastische vakanties voordat Jara en Torben er waren en de tijd dat we in Bunde woonden toen we ons huis verbouwde in Maastricht. Bij jullie ben ik altijd welkom.

Lieve Astrid, Hilde en Casper, wat is het heerlijk om in een groot gezin op te groeien. Astrid, sinds we allebei moeder zijn, is onze band enorm gegroeid, vele tips wisselen we uit. Met de vierde opkomst ben jij inmiddels ook echt een ervaringsdeskundige. Hilde, we lijken veel op elkaar en kunnen elkaar dan ook goed een spiegel voor houden. Ik geniet van onze gesprekken. De afgelopen jaren heb je het niet makkelijk gehad, maar inmiddels straal je weer, houd het vast. Casper, jij was altijd mijn kleine broertje, maar vandaag heb ik een enorme kerel naast mij staan als paranifm en dat voelt heel erg goed. Je staat vol zelfvertrouwen in het leven en kan ik erg genieten van je sterke verhalen. Jeroen, fijn dat jij inmiddels ook al zolang deel uit maakt van de familie. En Arjen, welkom bij de club.

Pap en mam, wat hebben jullie mij een geweldige basis gegeven om mijn leven op te bouwen. Ik ervaar eindeloos veel liefde en vertrouwen al wordt dat niet altijd uitgesproken. Jullie mogen heel erg trots zijn, want het is zeker mede aan jullie te danken dat ik dit bereikt heb.

Allerliefste Michiel, wij zijn een geweldig team! Het leven samen met jou is een feest en er komen steeds meer slingers bij. Bedankt voor de grenzeloze steun in de afgelopen jaren, zonder was het zeker niet gelukt om dit project tot een goed einde te brengen. Ik kijk alweer uit naar het volgende project. En dan zijn de allerlaatste woorden voor mijn kleine onderzoekers. Lieve Jara en lieve Torben, mama heeft weer alle tijd om met jullie de wereld te gaan ontdekken, gaan jullie mee? 

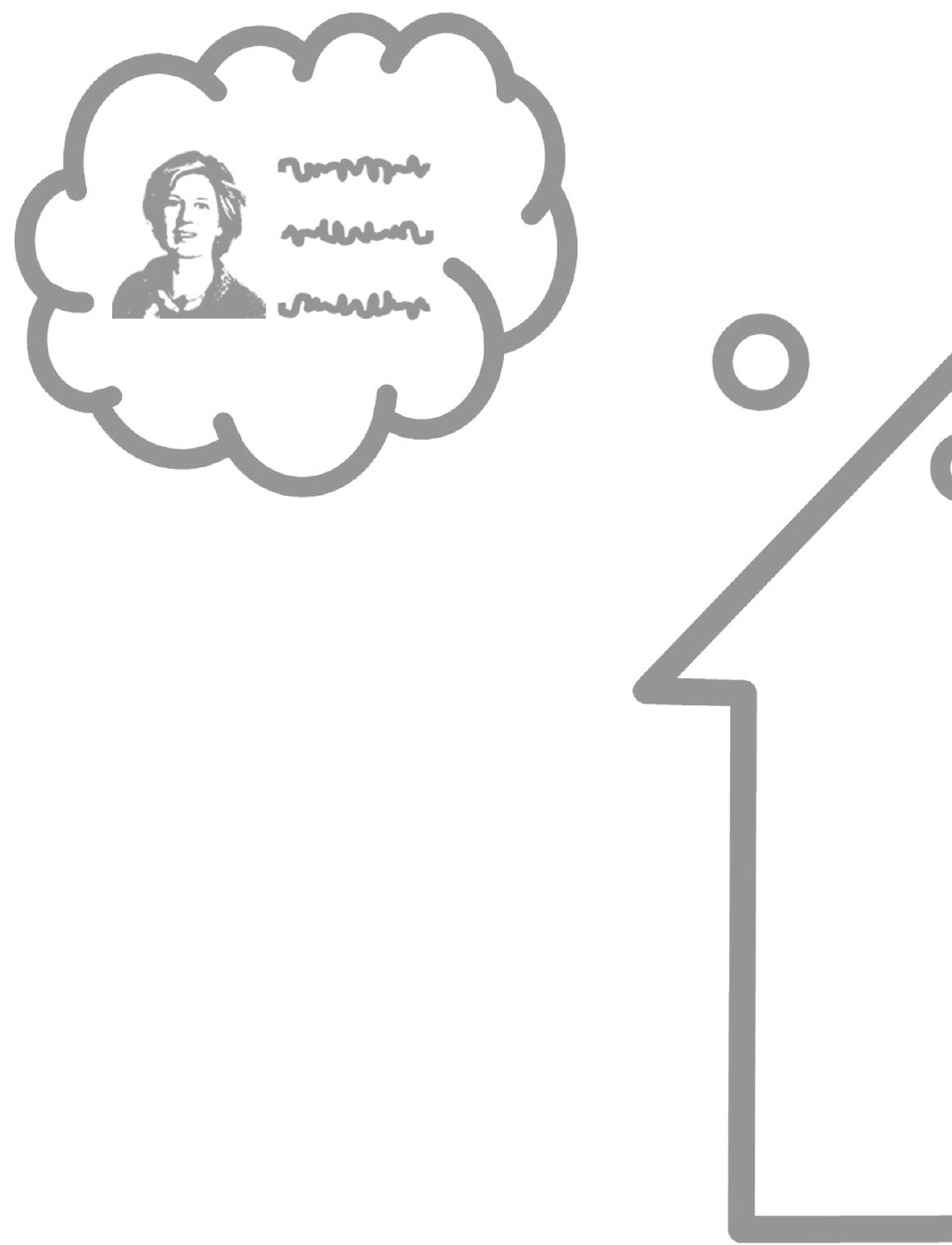
About the author 



\section{About the author}

Tanja Dorresteijn was born on the $29^{\text {th }}$ of October 1981 in Utrecht (the Netherlands). A year after completing her secondary school Montessori Lyceum Herman Jordan in Zeist (the Netherlands), she started her study psychology at Maastricht University. In 2005, she graduated on an evaluation study of the effect of fear appeals on smoking behavior in adolescents at the department of Work and Social Psychology in Maastricht. After a follow-up study of one year at the same department, she started in 2008 with her PhD trajectory in Maastricht at the department of Health Services Research. During this PhD trajectory, which was embedded in the research program Ageing and Long-Term Care of the School for Public Health and Primary Care (CAPHRI), she presented her research results at several international and national conferences. Tanja started in 2014 as consultant Health and Safety at the Public Health Services in Limburg-Noord (the Netherlands). She advices municipalities on population wide prevention in the social domain. Tanja is married to Michiel Ritzen and is mother of Jara (2011) and Torben (2014). 

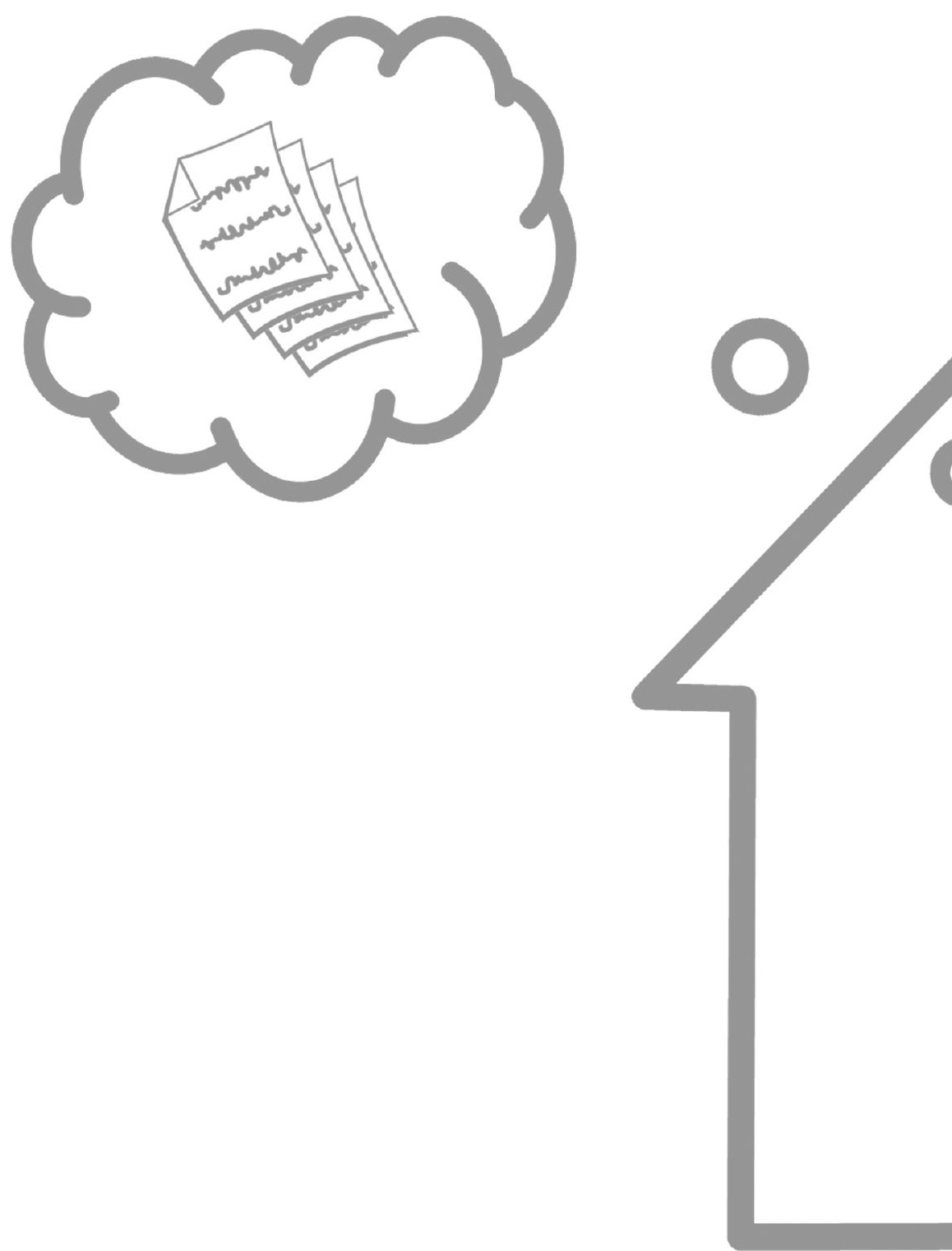
Publications 



\section{Publications}

\section{Articles}

Dorresteijn TAC, Zijlstra GAR, Van Haastregt JCM, Evers, SMAA, Kempen GIJM:

Cost-effectiveness of a home-based programme to reduce concerns about falls in frail, community-dwelling older people: evidence from a randomised controlled trial (submitted).

Dorresteijn TAC, Zijlstra GAR, Ambergen AW, Delbaere K, Vlaeyen JWS, Kempen GIJM: Effectiveness of a home-based cognitive behavioral program to manage concerns about falls in community-dwelling, frail older people: results of a randomized controlled trial. BMC Geriatr. 2016, 16(1):2.

Dorresteijn TAC, Zijlstra GAR, Van Haastregt JCM, Vlaeyen JWS, Kempen GIJM: Feasibility of a nurse-led in-home cognitive behavioral program to manage concerns about falls in frail older people: a process evaluation. Res Nurs Health. 2013, 36(3):257-270.

Dorresteijn TAC, Zijlstra GAR, Van Eijs YJ, Vlaeyen JWS, Kempen GIJM: Older people's preferences regarding programme formats for managing concerns about falls. Age Ageing. 2012, 41(4):474-481.

Dorresteijn TAC, Zijlstra GAR, Delbaere K, Van Rossum E, Vlaeyen JWS, Kempen GIJM: Evaluating an in-home multicomponent cognitive behavioural programme to manage concerns about falls and associated activity avoidance in frail community-dwelling older people: Design of a randomised control trial [NCT01358032]. BMC Health Serv Res. 2011, 11:228.

\section{Published abstracts and presentations}

Dorresteijn TAC, Zijlstra GAR, Van Haastregt JCM, Vlaeyen JWS, Kempen GIJM: Preventie van bezorgdheid om te vallen en vermijdingsgedrag bij kwetsbare ouderen: praktische uitvoerbaarheid van een individuele cognitief-gedragsmatige interventie. Oral presentation at Geriatriedagen 's-Hertogenbosch, Netherlands, 2013. Awarded for the best oral presentation in the category nurses.

Dorresteijn TAC, Zijlstra GAR, van Haastregt JCM, Vlaeyen JWS, Kempen GIJM: Delivery of a Fear of Falling Program: Comparing Facilitators' Self-Reports to Audio Recordings. Gerontologist. 2012, 52:247-247. Oral presentation at GSA Annual Conference San Diego, US, 2012. 
Dorresteijn TAC, Zijlstra GAR, Van Haastregt JCM, Vlaeyen JWS, Kempen GIJM: Uitvoerbaarheid van een individuele cognitief-gedragsmatige cursus om bezorgdheid om te vallen en vermijding van activiteiten bij kwetsbare ouderen te verminderen. Tijdschr Gerontol Geriatr. 2012, 43(4):196. Oral presentation at NVG congres Ede, Netherlands, 2012.

Dorresteijn TAC, Zijlstra GAR, van Haastregt JCM, Vlaeyen JWS, Kempen GIJM: Comparing Participants' and Trainers' Perceptions on Benefits of a Concerns About Falling Program. Gerontologist. 2011, 51:529-529. Presented by R. Zijlstra at GSA Annual Conference Boston, US, 2011.

Dorresteijn TAC, Zijlstra GAR, Van Eijs YJ, Vlaeyen JWS, Kempen GIJM: Preferences Regarding Intervention Formats of Community-Dwelling Older Adults with Fear of Falling. Gerontologist. 2010, 50:152-153. Oral presentation at GSA Annual Conference New Orleans, US, 2010.

Dorresteijn TAC, Zijlstra GAR, Vlaeyen JWS, Kempen GIJM: Ontwikkeling van een cognitief-gedragsmatige cursus om bezorgdheid om te vallen bij kwetsbare ouderen te verminderen en zelfredzaamheid te verbeteren. Tijdschr Gerontol Geriatr. 2010, 41(5):7. Oral presentation at NVG congres Ede, Netherlands, 2010.

Dorresteijn TAC, Zijlstra GAR, Vlaeyen JWS, Kempen GIJM: Managing fear of falling: the development of an in-home intervention for frail older people. Journal of Nutrition, Health and Aging. 2009, 13(S3):18. Poster presentation at IAGG World Congress Paris, France, 2009.

Dorresteijn TAC, Zijlstra GAR, Vlaeyen JWS, Kempen GIJM: Vermindering van psychisch disfunctioneren en angst om te vallen bij kwetsbare ouderen: ontwikkeling van een cognitief-gedragsmatige training. Tijdschr Gerontol Geriatr. 2008, 39:43. Poster presentation at NVG congres Ede, Netherlands, 2008. 


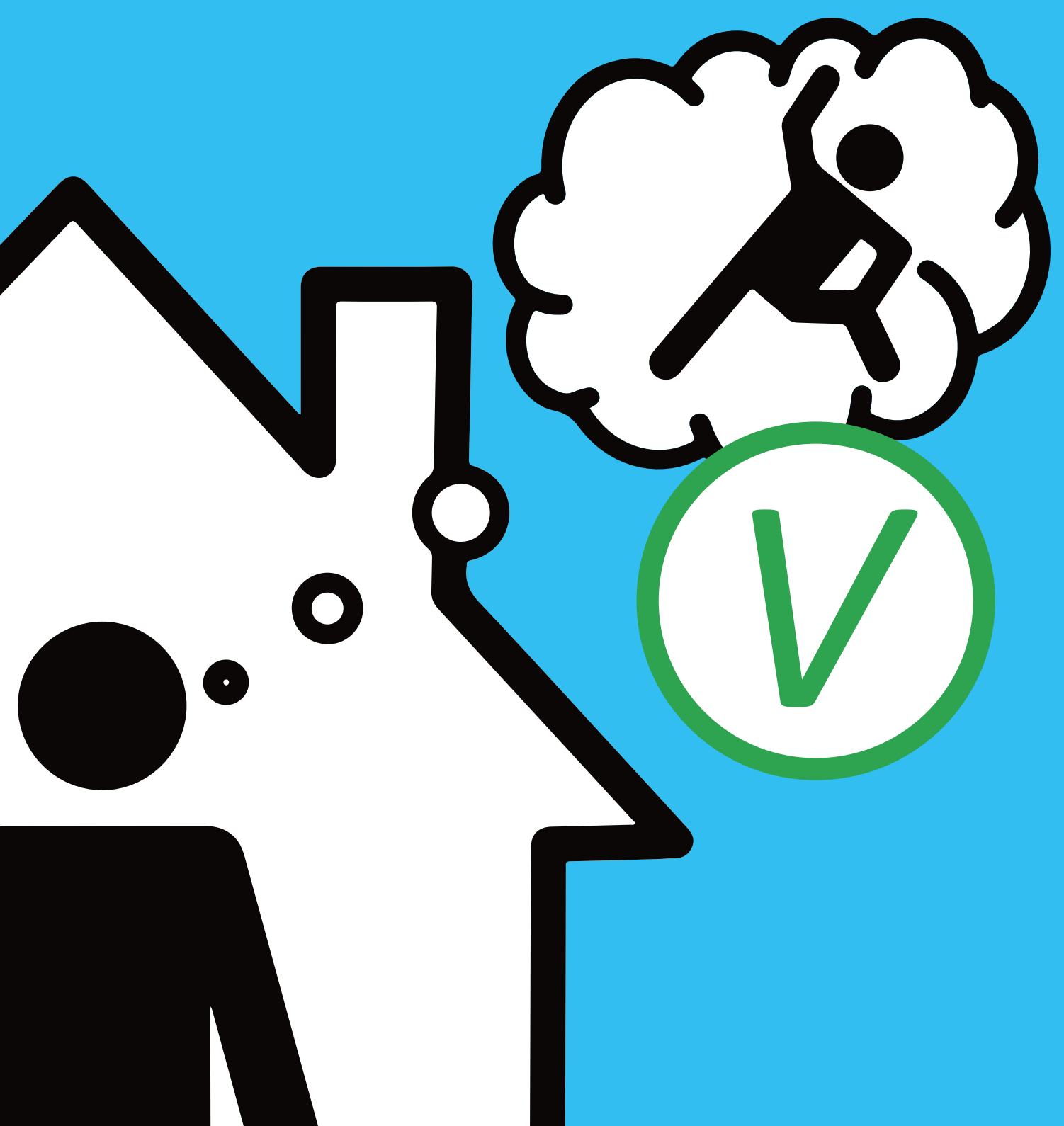

